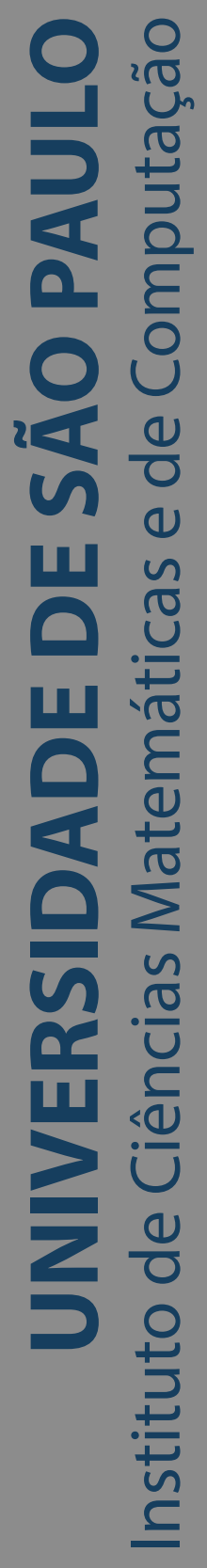

\title{
Multilevel method in bipartite networks
}

\section{Alan Demetrius Baria Valejo}

Tese de Doutorado do Programa de Pós-Graduação em Ciências de Computação e Matemática Computacional (PPG-CCMC) 

SERVIÇO DE PÓS-GRADUAÇÃO DO ICMC-USP

Data de Depósito:

Assinatura:

\section{Alan Demetrius Baria Valejo}

\section{Multilevel method in bipartite networks}

Doctoral dissertation submitted to the Institute of Mathematics and Computer Sciences - ICMC-USP, in partial fulfillment of the requirements for the degree of the Doctorate Program in Computer Science and Computational Mathematics. FINAL VERSION

Concentration Area: Computer Science and Computational Mathematics

Advisor: Prof. Dr. Alneu de Andrade Lopes Co-advisor: Profa. Dra. Maria Cristina Ferreira de Oliveira 
Ficha catalográfica elaborada pela Biblioteca Prof. Achille Bassi e Seção Técnica de Informática, ICMC/USP, com os dados inseridos pelo(a) autor(a)

Valejo, Alan Demetrius Baria Método multinível em redes bipartidas / Alan Demetrius Baria Valejo; orientador Alneu de Andrade Lopes; coorientadora Maria Cristina Ferreira de Oliveira. -- São Carlos, $223 \mathrm{p}$.

Tese (Doutorado - Programa de Pós-Graduação em Ciências de Computação e Matemática Computacional) Instituto de Ciências Matemáticas e de Computação, Universidade de São Paulo, .

1. INTELIGÊNCIA ARTIFICIAL. 2. MINERAÇÃODEDADOS. 3. REDES COMPLEXAS. 4. REDES BIPARTIDAS. 5. MÉTODO MULTINÍVEL. I. Lopes, Alneu de Andrade, orient. II. Oliveira, Maria Cristina Ferreira de, coorient. III. Título.

Bibliotecários responsáveis pela estrutura de catalogação da publicação de acordo com a AACR2: Gláucia Maria Saia Cristianini - CRB - 8/4938

Juliana de Souza Moraes - CRB - 8/6176 


\section{Alan Demetrius Baria Valejo}

\section{Método multinível em redes bipartidas}

Tese apresentada ao Instituto de Ciências Matemáticas e de Computação - ICMC-USP, como parte dos requisitos para obtenção do título de Doutora em Ciências - Ciências de Computação e Matemática Computacional. VERSÃO REVISADA

Área de Concentração: Ciências de Computação e Matemática Computacional

Orientador: Prof. Dr. Alneu de Andrade Lopes

Coorientadora: Profa. Dra. Maria Cristina Ferreira de Oliveira 

Obrigado meu amado Jesus por ser meu conforto e, sobretudo, por seu amor incondicional e misericórdia.

Agradeço à minha família, em especial, meus pais Elzio e Aparecida e a minha namorada Wiviane, por todo o amor, apoio e compreensão que sempre forneceram.

Gostaria de expressar meus sinceros agradecimentos e gratidão ao professor Alneu de Andrade Lopes e à professora Maria Cristina Ferreira de Oliveira, meus orientadores ao longo desses anos de pesquisa. Destaco os conselhos acadêmicos e pessoais, que foram essenciais para a minha formação. Eu aprendi muito nesses anos da minha jornada acadêmico/científica e sempre serei grato por toda a ajuda, incentivo e amizade.

Agradeço aos amigos do LABIC por deixarem uma marca especial na minha vida.

Agradeço o apoio financeiro da agência brasileira CAPES, Coordenação de Aperfeiçoamento de Pessoal de Nível Superior, para o desenvolvimento desta tese. 



\section{ABSTRACT}

VALEJO, A. D. B. Multilevel method in bipartite networks. 2019. 223 p. Tese (Doutorado em Ciências - Ciências de Computação e Matemática Computacional) - Instituto de Ciências Matemáticas e de Computação, Universidade de São Paulo, São Carlos - SP, 2019.

Bipartite networks comprise a particular class of network models in which the vertex set is split into two disjoint and independent subsets, with edges connecting only vertices placed in different subsets. They provide a powerful representation of the relationships in many realworld systems and have been widely employed to model data-intensive problems. In a related scenario, multilevel methods have been previously applied to handle computationally expensive optimization problems defined in networks. The strategy aims at reducing the cost of executing an expensive algorithm or task by exploiting a hierarchy of coarsened versions of the original network. There is a growing interest in multilevel methods in networked systems, motivated mostly by their capability of handling large-scale networks and applicability to a variety of problems, most notably community detection and network drawing. Despite their potential, existing approaches are not directly applicable to bipartite networks and, to the best of our knowledge, the multilevel strategy had not been considered in this context so far, opening a vast space for scientific exploration. This gap motivated this research project, which introduces a study on multilevel methods applicable to bipartite networks. In order to overcome the aforementioned limitations, this thesis presents two novel multilevel frameworks for handling bipartite structures, named $O P M$ and $M O b . O P M$ analyzes the bipartite network based on its one-mode projections, allowing the reuse of classical and already established solutions from the literature. $M O b$ (and its $M d r, C S V$ and $C S L$ variations) operate directly on the bipartite representation to execute the multilevel method, providing a cost-effective implementation. Empirical results obtained on a set of synthetic and real-world networks on diverse applications indicate a considerable speed up with no significant loss in the quality of the solutions obtained in the coarsened networks as compared to those obtained in the original network (i.e., conventional approaches). The potential applicability and reliability of the proposed methods have been illustrated in multiple scenarios, namely optimization, community detection, dimensionality reduction and visualization. Furthermore, the results provide empirical evidence that the proposed methods can foster novel applications of the multilevel method in bipartite networks, e.g. link prediction and trajectory mining and, therefore, that this thesis brings a relevant contribution to the field.

Keywords: Complex networks, bipartite networks, large-scale networks, multilevel method, network coarsening. 



\section{RESUMO}

VALEJO, A. D. B. Método multinível em redes bipartidas. 2019. 223 p. Tese (Doutorado em Ciências - Ciências de Computação e Matemática Computacional) - Instituto de Ciências Matemáticas e de Computação, Universidade de São Paulo, São Carlos - SP, 2019.

As redes bipartidas compreendem uma classe particular das redes complexas, na qual vértices são divididos em dois subconjuntos separados e independentes e as arestas conectam apenas vértices de conjuntos diferentes. Tais redes fornecem uma poderosa representação para a modelagem de muitos sistemas complexos do mundo real e têm sido amplamente empregadas em problemas caracterizados pelo alto custo computacional e uso intensivo de dados. Nessa linha, os chamados métodos multinível têm sido empregados para tratar problemas computacionalmente custosos e descrevem uma estratégia escalável que explora (e cria) uma hierarquia de versões reduzidas, ou simplificadas, da rede original. Nos últimos anos, houve um crescente interesse em métodos multinível motivado, principalmente, por sua capacidade de manipular redes de larga escala, bem como sua aplicabilidade em diversos problemas, como detecção de comunidades e visualização. Apesar de seu potencial, as abordagens atuais não são diretamente aplicáveis às redes bipartites e, até onde sabemos, a estratégia multinível não havia sido considerada neste contexto anteriormente, abrindo um vasto espaço para exploração científica. Essa lacuna motivou este projeto de pesquisa, o qual introduz um estudo sobre métodos multinível aplicáveis às redes bipartidas. Para superar as limitações mencionadas, esta tese apresenta duas novas estratégias direcionadas às redes bipartidas, denominadas $O P M$ e $M O b$. O $O P M$ analisa a rede bipartida em suas projeções unipartidas e permite a reutilização de algoritmos multinível clássicos e já estabelecidos na literatura. O $M O b$ (e suas variações $M d r, C S V$ e $C S L$ ) considera diretamente a estrutura bipartida para executar o método multinível e fornecer uma implementação eficiente e eficaz. Os resultados empíricos obtidos em conjuntos de redes reais e sintéticas, em uma variedade de aplicações, demonstram uma redução considerável no tempo de processamento sem perda significativa na qualidade da solução obtida na rede reduzida, quando comparada aos resultados obtidos na rede original. A potencial aplicabilidade e confiabilidade dos métodos propostos foram ilustradas em múltiplos cenários, a saber: otimização, detecção de comunidades, redução de dimensionalidade e visualização. Além disso, os resultados fornecem evidências empíricas de que os métodos propostos podem fomentar novas aplicações do método multinível em redes bipartidas, por exemplo, na predição de arestas e mineração de trajetórias, e evidenciam que este estudo gerou contribuições relevantes para a área.

Palavras-chave: Redes complexas, redes bipartidas, redes de grande escala, método multinível, contração de redes. 

Figure 1 - Number of publications per year, from 1992 to December 2018 . . . . . . . 38

Figure 2 - Distribution of the papers selected. . . . . . . . . . . . . . 40

Figure 3 - Walshaw's and Karypis's co-authorship networks . . . . . . . . . . 42

Figure 4 - Phases of a multilevel method. . . . . . . . . . . . . . . 44

Figure 5 - Two examples of matching. . . . . . . . . . . . . . 47

Figure 6 - Edge-selection matching (ESM) strategy . . . . . . . . . . . . . . . 48

Figure 7 - Edge-selection strategy. . . . . . . . . . . . . . . . . . 49

Figure 8 - Prohibitive matching case. . . . . . . . . . . . . . . . . 49

Figure 9 - Randomly matching. . . . . . . . . . . . . 50

Figure 10 - Vertex-selection matching strategy. . . . . . . . . . . . . . . . 50

Figure 11 - Local 2-hop neighborhood selection. . . . . . . . . . . . . . . . 50

Figure 12 - Edge and vertex-selection matching strategies. . . . . . . . . . . . . 51

Figure 13 - Cluster-selection matching strategy. . . . . . . . . . . . . . 52

Figure 14 - Different edge-selection matching (ESM) strategies. . . . . . . . . . . 53

Figure 15 - Difference between the GVM and HEM matching algorithms. . . . . . . . . 54

Figure 16 - Variations of the VSM matching: a and b depict the classic and the star-like approaches, respectively. . . . . . . . . . . . . . . 5 55

Figure 17 - Vertex-selection matching. . . . . . . . . . . . . . . 56

Figure 18 - Example of matching in a bipartite network . . . . . . . . . . 56

Figure 19 - Cluster-selection matching based on label propagation . . . . . . . . . . 58

Figure $20-$ Size-constraint super-vertex . . . . . . . . . . . . . . . 58

Figure 21 - Result from a coarsening process (Pairwise) . . . . . . . . . . . . . 60

Figure 22 - Contraction of clustering or multigraphs. . . . . . . . . . . . 61

Figure 23 - Contraction process of a hypergraph. . . . . . . . . . . . 62

Figure 24 - Local minimum problem. . . . . . . . . . . . . . . . 63

Figure 25 - K-way Kernighan-Lin refinement. . . . . . . . . . . . . . . 65

Figure 26 - Difference between a modularity-based and KK algorithms. . . . . . . . . 65

Figure 27 - Multilevel visualization . . . . . . . . . . . . . . . . 67

Figure 28 - Multilevel community detection. . . . . . . . . . . . . . . 68

Figure 29 - Multilevel dimensionality reduction. . . . . . . . . . . . . . . . . 69

Figure 30 - Multilevel graph coloring. . . . . . . . . . . . . . . . . . 69

Figure 31 - Negative binomial distribution for distinct values of parameters $m u$ and $d . \quad$. 91

Figure 32 - Bipartite networks with distinct topological properties. . . . . . . . . . 93 
Figure 33 - Views of the adjacency matrices. . . . . . . . . . . . . . . . 94

Figure $34-$ Behavior of a network's average degree . . . . . . . . . . . . . . 95

Figure 35 - Behavior of a network's average clustering coefficient. . . . . . . . . . . 96

Figure 36 - istribution of vertex degree and local clustering coefficient. . . . . . . . . 96

Figure 37 - Bipartite networks generated with BNOC . . . . . . . . . . . . 97

Figure 38 - Bipartite network generated with BNOC . . . . . . . . . . . . . . . 98

Figure 39 - Number of edges (sparsity) as a function of the dispersion parameter $d$. . . 99

Figure 40 - Runtime as a function of the number of vertices $v \ldots \ldots$. . . . . . . 100

Figure $41-$ Runtime as a function of the number of communities $c$. . . . . . . . 100

Figure 42 - Runtimes for obtaining synthetic networks. . . . . . . . . . . . . . . 101

Figure 43 - NMI results for HLC and OSLOM. . . . . . . . . . . . . . . . . . 103

Figure 44 - Acc values for HLC as OSLOM. . . . . . . . . . . . . . . . . . . 104

Figure 45 - HLC and OSLOM runtimes. . . . . . . . . . . . . . . 106

Figure 46 - Two equivalent sets of synthetic networks. . . . . . . . . . . . . . 107

Figure 47 - NMI values for biSBM. . . . . . . . . . . . . . . . . . . . . . . 109

Figure 48 - Network schemas. . . . . . . . . . . . . . . . . . . . . . 111

Figure 49 - A HIN described as $r$ bipartite/homogeneous networks. . . . . . . . . . . . 112

Figure 50 - Heterogeneous networks generated with HNOC. . . . . . . . . . . . 113

Figure 51 - A bipartite network . . . . . . . . . . . . . . . . . . 118

Figure 52 - Unipartite networks $G^{1}$ and $G^{2} \ldots \ldots \ldots \ldots \ldots$

Figure 53 - A weighted projection. . . . . . . . . . . . . . 118

Figure 54 - Super-vertex example. . . . . . . . . . . . . . . . . . 120

Figure 55 - Nemenyi post-hoc test. . . . . . . . . . . . . . . . . . . . . 124

Figure 56 - Averages and standard deviations of the NMI accuracy values. . . . . . . 125

Figure 57 - Shape distribution, variability, and averages of the accuracy values. . . . . . 126

Figure 58 - Phases of a multilevel method. . . . . . . . . . . . . . 133

Figure 59 - Example of a bipartite network. . . . . . . . . . . . . . . 136

Figure 60 - Bipartite network. . . . . . . . . . . . . . . . . . . 138

Figure 61 - Bipartite network. . . . . . . . . . . . . . . . . . . 139

Figure 62 - Contraction of a layer with the $G M b_{c n}$ matching algorithm. . . . . . . . . 141

Figure 63 - Scale-free properties of the Cond-Mat bipartite network. . . . . . . . . . . . 144

Figure 64 - Percentages of remaining vertices and edges in the networks. . . . . . . . . 144

Figure 65 - Degree distribution. . . . . . . . . . . . . . . . . . . . . 145

Figure 66 - Two-mode local clustering coefficient. . . . . . . . . . . . . . . . 145

Figure 67 - Averages and standard deviations of the NMI accuracy. . . . . . . . . . . 149

Figure 68 - Shape distribution, variability, and median of the accuracy values. . . . . . . 149

Figure 69 - Nemenyi post-hoc test for $L P A w b+$ and the four $M O b$ instances. . . . . . . 150

Figure 70 - Contribution of each algorithm to the total time of the experiments. . . . . 151

Figure 71 - Relative contribution of each multilevel phase to the execution time. . . . . 152 
Figure 72 - Average percentage of execution time) . . . . . . . . . . . . . . 153

Figure 73 - Performance (accuracy values) of the $M d r-k N N$ classifier. . . . . . . . . . 154

Figure 74 - Nemenyi post-hoc test for $k N N \ldots \ldots \ldots$. . . . . . . . . . . 156

Figure 75 - Nemenyi post-hoc test for $M O b$ and $O P M$ version of the $L P A w b+\ldots$. . . . 159

Figure 76 - Phases of a multilevel method. . . . . . . . . . . . . . . 165

Figure 77 - OPM matching of a bipartite network. . . . . . . . . . . . . 167

Figure $78-R G M b$ matching for bipartite networks. . . . . . . . . . . . 168

Figure 79 - Matching example. . . . . . . . . . . . . . . . 168

Figure $80-M O b$ priority choices. . . . . . . . . . . . . . . . . . . 169

Figure 81 - Impact of the upper bound. . . . . . . . . . . . . . . . 171

Figure 82 - A coarsened bipartite network. . . . . . . . . . . . . 175

Figure 83 - Average number of coarsening levels L . . . . . . . . . . . . . . . . 177

Figure 84 - Number of iterations $T$ required for $M L P b$ to converge. . . . . . . . . . . 177

Figure 85 - NMI accuracy values of biSBM . . . . . . . . . . . . . . . . . 180

Figure 86 - Nemenyi post-hoc test for biSBM. . . . . . . . . . . . . . . . 181

Figure 87 - Absolute and relative runtime. . . . . . . . . . . . . . . . . 182

Figure 88 - Average runtime. . . . . . . . . . . . . . . . 183

Figure 89 - Absolute runtime. . . . . . . . . . . . . . . . . . . . 184

Figure 90 - Critical difference diagram of the results in Table 19. . . . . . . . . . 186

Figure 91 - Multiple layouts of a synthetic sparse bipartite network. . . . . . . . . . . 189

Figure 92 - Synthetic bipartite network. . . . . . . . . . . . . . . . . . 190

Figure 93 - Moreno Crime social network. . . . . . . . . . . . . . . . . . . 191

Figure 94 - N-reatome biological network. . . . . . . . . . . . . . . . . 192

Figure 95 - Drawings of the N-reatome biological network after coarsening process. . . 193

Figure 96 - Bipartite network three holes structure. . . . . . . . . . . . . . . 194 

Algorithm 1 - Multilevel method framework. . . . . . . . . . . . . . 45

Algorithm 2 - Contraction of a network from a cluster-selection matching. . . . . . . 60

Algorithm 3 - Projection of a community structure. . . . . . . . . . . . 63

Algorithm 4 - Basic structure of matching. . . . . . . . . . . . . 75

Algorithm 5 - Greedy matching. . . . . . . . . . . . . . 75

Algorithm 6 - General algorithm for path growing based matching. . . . . . . . . 76

Algorithm 7 - Alternating non-negative least squares. . . . . . . . . . . . 78

Algorithm 8 - Matching for bipartite networks. . . . . . . . . . . . . . . 79

Algorithm 9 - Local greedy matching based on modularity maximization. . . . . . . . 80

Algorithm 10 - Greedy matching based on merge prioritizer. . . . . . . . . . . . 81

Algorithm 11 - Matching based on label propagation. . . . . . . . . . . . . . . 81

Algorithm 12 - Kernighan-Lin refinement algorithm . . . . . . . . . . . . . 82

Algorithm 13 - OPM: One-mode projection-based multilevel community detection . . 121

Algorithm 14 - MOb: multilevel optimization framework for bipartite networks. . . . 136

Algorithm 15 - RGMb: random greedy matching for bipartite networks. . . . . . . . 137

Algorithm 16 - GMb: greedy matching for bipartite networks. . . . . . . . . . . . 139

Algorithm 17 - Cb: Contraction of a bipartite network from a matching . . . . . . . 140

Algorithm 18 - Ub: Uncoarsening of bipartite networks. . . . . . . . . . . . 141

Algorithm 19 - Mdr: multilevel dimensionality reduction. . . . . . . . . . . . . . 154

Algorithm $20-M L P b$ : Matching via weight-constrained label propagation. . . . . . 172

Algorithm $21-C b$ : Contraction of a bipartite network. . . . . . . . . . . . 173

Algorithm 22 - $M L P b_{C S L}$ : Coarsening with number of coarsening levels. . . . . . . . 174

Algorithm $23-M L P b_{C S V}$ : Coarsening with minimum number of vertices as input. . . 174 

Table 1 - Keywords, synonyms and related terms used to search articles. . . . . . . . 36

Table 3 - Top 5 cited papers. . . . . . . . . . . . . . . . 39

Table 4 - Top 5 cited papers in the last ten years $(2008-2018)$. . . . . . . . . . . 39

Table 7 - Description of the controlling parameters in BNOC. . . . . . . . . . 90

Table 8 - Normalized mutual information for biSBM. . . . . . . . . . . . . 108

Table 9 - Modified and additional parameters in HNOC. . . . . . . . . . . . . . 112

Table 10 - NMI accuracy values of the three $O P M$ instantiations. . . . . . . . . . . . . . . . . . . . . . . . . 123

Table 11 - Absolute runtime of $L P A w b+$ and four $O P M$ instances. . . . . . . . . . . . 126

Table 12 - MOb applied to target algorithm $L P A w b+\ldots \ldots \ldots$. . . . . . . . . . . . 147

Table 13 - NMI accuracy values of all MOb instances and baseline $L P A w b+$. . . . . . . 148

Table 14 - Absolute runtime of $L P A w b+$ and four $M O b$ instances. . . . . . . . . . . . 151

Table 15 - Properties of text collections considered. . . . . . . . . . . . . . 154

Table 16 - Accuracy values obtained with standard $k N N, M d r-k N N$ and PCA-kNN . . . 155

Table 17 - NMI accuracy values of the $M O b$ and $O P M$ instantiations. . . . . . . . . . . 159

Table 18 - Properties of the largest connected component. . . . . . . . . . . . . 185

Table $19-C$ scores. . . . . . . . . . . . . . . . . . . . 186

Table 20 - Algorithm execution times. . . . . . . . . . . . . . 187 



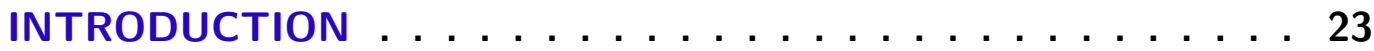

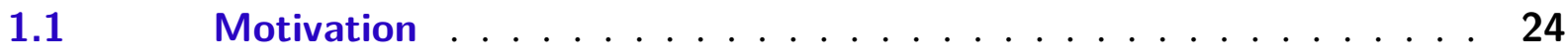

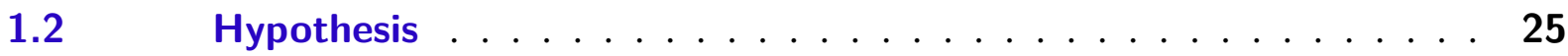

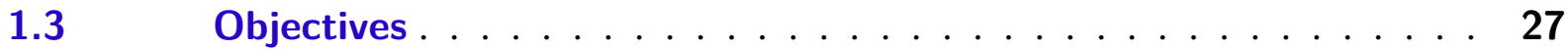

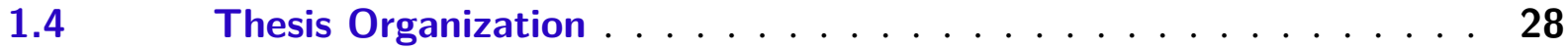

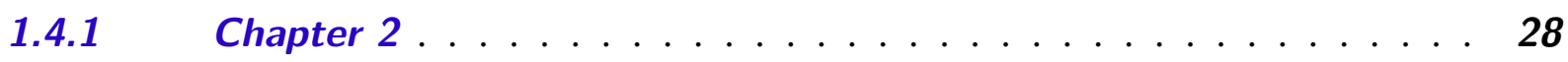

1.4.2 Chapter $3 \ldots \ldots \ldots \ldots \ldots$

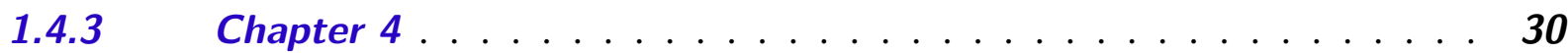

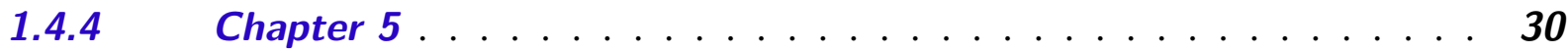

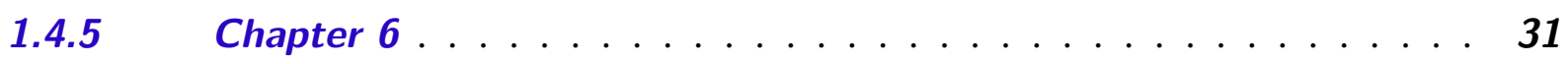

1.4.6 Conclusion ....................... 31

2 A CRITICAL SURVEY OF THE MULTILEVEL METHOD IN COMPLEX NETWORKS . . . . . . . . . . . . . . . . 33

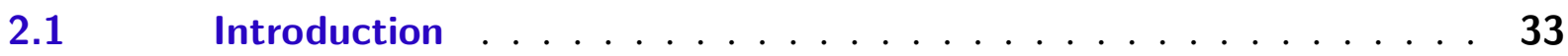

2.2 A panorama of research contributions on multilevel method . . . 35

$2.3 \quad$ Basic Concepts . . . . . . . . . . . . . . . . . . . . 42

$2.4 \quad$ Multilevel method framework . . . . . . . . . . . . . . . 44

$2.5 \quad$ Coarsening . . . . . . . . . . . . . . 46

2.5.1 Matching . . . . . . . . . . . . . . . 47

2.5.1.1 Edge-selection matching algorithms $(E S M) \ldots \ldots \ldots 2$

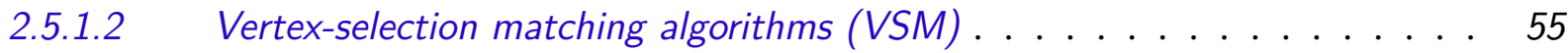

2.5.1.3 Cluster-selection matching algorithms (CSM) . . . . . . . . . 56

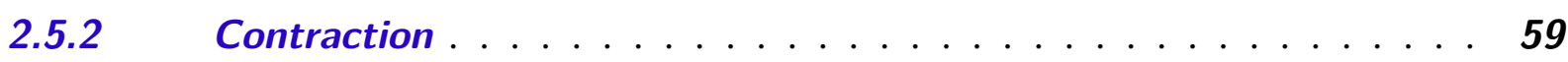

$2.6 \quad$ Uncoarsening . . . . . . . . . . . . . . . 62

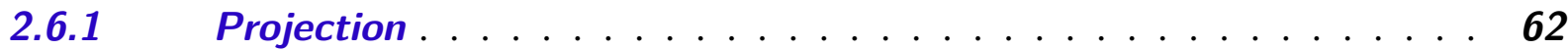

2.6.2 Refinement .................... 63

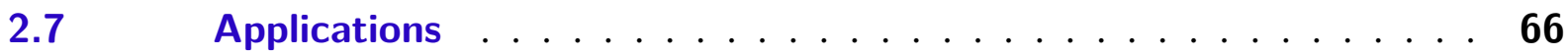

2.7.1 Visualization . . . . . . . . . . . . . . . 66

2.7.2 Community detection . . . . . . . . . . . . . . . . . . . 66

2.7.3 Dimensionality reduction . . . . . . . . . . . . . . . . 68

2.7.4 Graph coloring . . . . . . . . . . . . . . . . . . 68

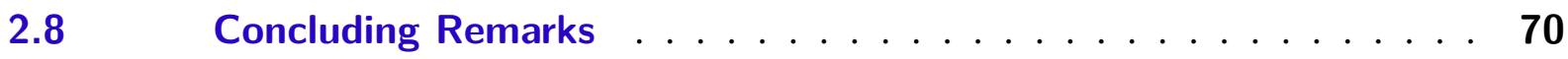




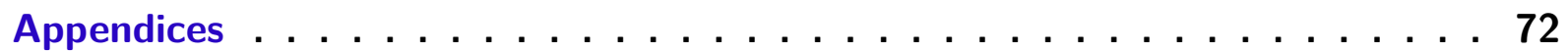

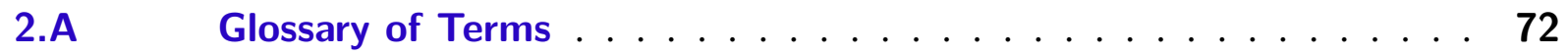

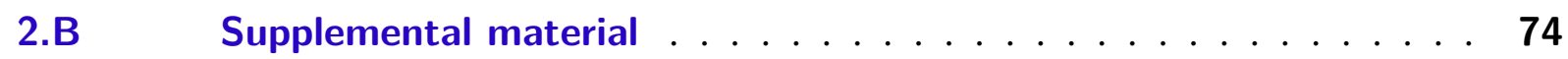

2.B.1 Standard matching algorithms . . . . . . . . . . . . 74

2.B.2 Greedy heavy edge matching algorithm GHEM _. . . . . . . . . 74

2.B.3 Path growing matching algorithms . . . . . . . . . . . 75

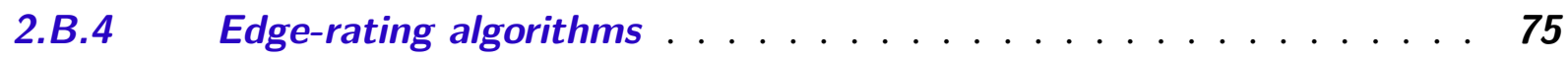

2.B.5 Vertex-selection matching for bipartite networks . . . . . . . 79

2.B.6 Greedy matching based on modularity maximization . . . . . . . 79

2.B.7 Kernighan-Lin (KL) refinement algorithm . . . . . . . . . 82

3 A BENCHMARKING TOOL FOR THE GENERATION OF BIPARTITE NETWORK MODELS WITH OVERLAPPING COMMUNI-

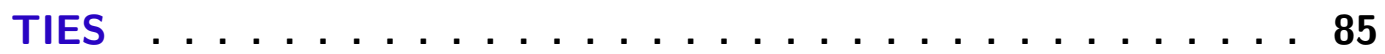

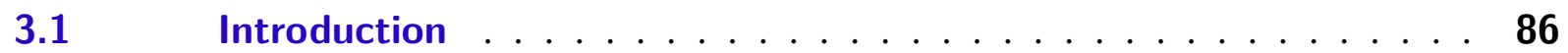

$3.2 \quad$ Related Work . . . . . . . . . . . . . . . . . 88

3.3 A Tool for Benchmark Generation . . . . . . . . . . . . . . 89

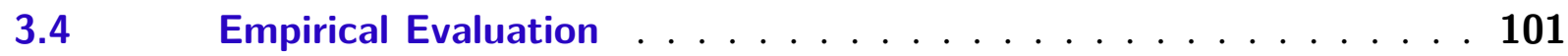

3.4.1 Support for consistent algorithm evaluation . . . . . . . . . . 102

3.4.2 Comparison with existing benchmarks . . . . . . . . . . . . . 105

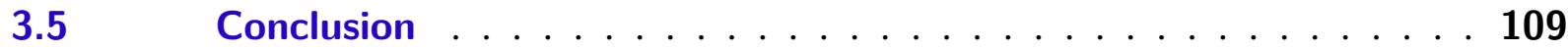

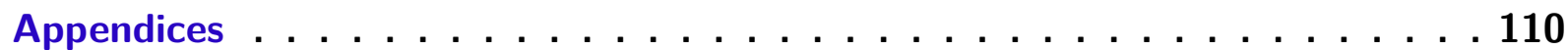

3.A HNOC: Extension to $k$-partite and heterogeneous networks . . . 110

4 COMMUNITY DETECTION IN BIPARTITE NETWORK: A MODIFIED COARSENING APPROACH $\ldots \ldots \ldots \ldots \ldots$

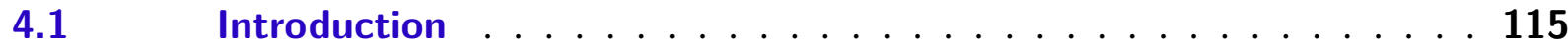

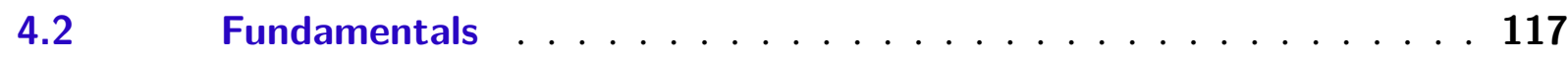

4.2.1 Basic definitions . . . . . . . . . . . . . . . . . . . 117

4.2.2 Multilevel method . . . . . . . . . . . . . . . . . . . . 119

4.3 Community detection based on multilevel method by using one-

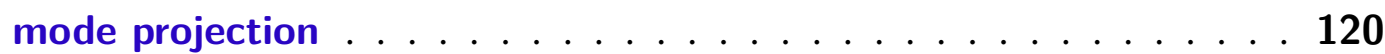

$4.4 \quad$ Experimental Results and Analysis . . . . . . . . . . . 122

$4.5 \quad$ Conclusions . . . . . . . . . . . . . . . . . . . . . . . . . . . 127

5 MULTILEVEL APPROACH FOR COMBINATORIAL OPTIMIZATION IN BIPARTITE NETWORKS . . . . . . . . . . . . 129

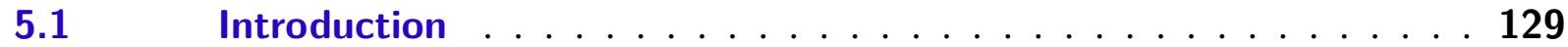

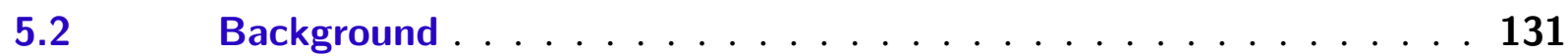

5.2.1 Basic concepts . . . . . . . . . . . . . . . . . 131 
5.2.2 General-purpose multilevel method . . . . . . . . . . . . . . . 132

$5.3 \quad$ Related Work . . . . . . . . . . . . . . . . . . . . 134

$5.4 \quad$ Multilevel method in bipartite networks . . . . . . . . . . . 135

5.4.1 Matching in bipartite networks . . . . . . . . . . . . . . 137

5.4.2 Contraction in bipartite networks . . . . . . . . . . . . . . . 139

5.4.3 Uncoarsening algorithm .................. 141

5.4.4 Computational complexity . . . . . . . . . . . . . . 142

$5.5 \quad$ Experimental Results and Analysis . . . . . . . . . . . . . . 143

5.5.1 Analysis of topological properties . . . . . . . . . . . . . . . . . 143

5.5.2 Performance in benchmark networks for community detection . . . 146

5.5.3 A test case on dimensionality reduction . . . . . . . . . . 153

5.6 The application of $M O b$ to other combinatorial optimization problems156

5.7 Conclusion and further research . . . . . . . . . . . 157

Appendices . . . . . . . . . . . . . . . . . . . . 158

5.A Supplemental material ................. 158

6 A COARSENING METHOD FOR LABELED AND UNLABELLED BIPARTITE NETWORK VIA WEIGHT-CONSTRAINED LABEL PROPAGATION ...................... . . . . . . . . . .

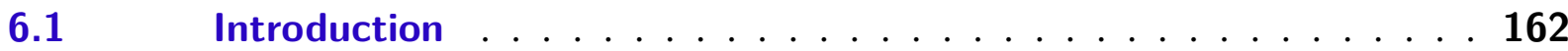

6.2 Background . . . . . . . . . . . . . . 163

6.2.1 Multilevel method in bipartite networks . . . . . . . . . . . . . . 164

6.2.2 Coarsening strategies and their limitations . . . . . . . . . . . . 166

6.3 Coarsening via weight-constraint and label propagation . . . . . 169

6.4 Parametric and complexity analysis . . . . . . . . . . 175

$6.5 \quad$ Experimental Results and Analysis . . . . . . . . . . . . . . 178

6.5.1 Benchmark and optimization problem . . . . . . . . . . . . 178

6.5.2 Community detection . . . . . . . . . . . . . . 183

6.5.3 Network coarsening for visualization . . . . . . . . . . . . . 187

$6.6 \quad$ Concluding Remarks . . . . . . . . . . . . . . . . 195

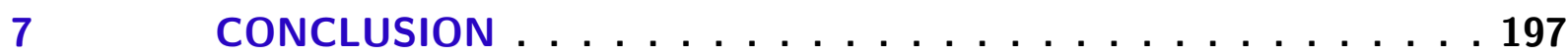

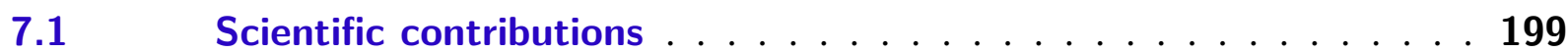

7.2 Limitations and possible research directions . . . . . . . . . . 201

BIBLIOGRAPHY . . . . . . . . . . . . . . . . . 203 

CHAPTER

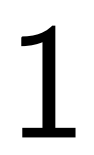

INTRODUCTION

Complex systems can be described as a set of units (or parts) that interact with each other and self-organize into components to create patterns of nontrivial connections and nonlinear behavior. They evolve without central control or hierarchy structure and engage into collective functionality (BAR-YAM, 2002). The field of complex networks emerged aimed at understanding their behavior, organization, evolution and dynamics, which represents these systems as non trivial graphs, where units and their relationships (or connections) are depicted as vertices and edges, respectively (WATTS; STROGATZ, 1998; NEWMAN, 2001a; NEWMAN, 2001b; NEWMAN, 2003; BORNHOLDT; SCHUSTER, 2003). Complex networks comprise a multidisciplinary research field, which spreads to many disciplines such as physics, engineering, biology, sociology and economics and has established itself as a successful approach to characterize, analyze, model and simulate complex systems, from the biological to the social and technological domains. Examples include social relationship networks (SCOTT, 2000), brain networks (SPORNS, 2002), metabolic networks (JEONG et al., 2000), the World Wide Web (ALBERT; JEONG; BARABASI, 1999) and the Internet (FALOUTSOS; FALOUTSOS; FALOUTSOS, 1999). Hereinafter complex networks will be referred to simply as networks.

Historically, the classical network modeling approach assumes only one type of edge and vertex and such networks are known as unipartite, or single-layer. However, this is a far too restrict assumption, since most real-world systems are organized into layers and formed by units (vertices) and interactions (edges) of distinct types. A social network, for instance, can consider different social relations, such as friendship, fellowship or family relatives, and different individuals, such as man and woman. Losing this heterogeneity can suppress useful (or intrinsic) features of an observed phenomena.

In this context, many real-world systems are naturally modeled as a bipartite, or twolayer, networks in which vertices are split into two disjoint sets (called layers) and an edge always connects vertices in different sets (LATAPY; MAGNIEN; VECCHIO, 2008; KITSAK; PAPADOPOULOS; KRIOUKOV, 2017). This type of network often results from modeling 
pairwise relationships between two categories of entities and have been successfully employed to model complex systems in diverse domains, such as documents and terms (FALEIROS; ROSSI; LOPES, 2017), patient and gene expression (or clinical variables) (HWANG et al., 2008), protein and ligand (JEONG et al., 2000), actors and movies (WATTS; STROGATZ, 1998), individuals and songs (or books, or films) (GRUJIĆ, 2008), geo referenced user-location (WANG et al., 2014b), and scientific papers and their authors (NEWMAN, 2001a; NEWMAN, 2001b).

\subsection{Motivation}

Many data-intensive machine learning tasks are performed on bipartite network models, in which reliable large-scale ${ }^{1}$ data are available, for instance community detection (or network partitioning) (VALEJO et al., 2018a) and classification (ROSSI et al., 2014). Solving these and other machine learning problems requires finding a minimum (or maximum) cost in a solution space wherein the number of possible states is finite, but often exponential. Indeed, many theoretical problems defined in bipartite networks are known to be $N P$-complete or $N P$-hard (ASRATIAN; DENLEY; HäGGKVIST, 1998). Therefore, scalability issues are widely studied in this context.

Following this trend, multilevel approaches (MA) have been employed to handle computationally expensive optimization problems defined in unipartite networks. The strategy aims at reducing the cost of executing a target algorithm (or the cost of solving an expensive task) over a given network by applying it to a coarsened (or reduced) version of the original network.

The strategy comprises three phases: coarsening, solving the problem, and uncoarsening. In the first phase, the input network is iteratively coarsened into a hierarchy of gradually smaller networks until a desired size is reached. Coarsening is split into matching, which selects pairs or groups of vertices to be matched, and contraction, which collapses the matched vertices and their edges into so-called super-vertices and super-edges, respectively. In the second phase, the problem is solved (using a high-cost target algorithm) in the coarsest network and an initial solution is obtained. Finally, in the third phase, the solution is successively projected (and refined) back over the inverse sequence of coarsened networks, until obtaining a final solution relative to the original network.

As shown in previous studies, the strategy enables running computationally expensive algorithms on large networks with no significant loss in solution quality (KARYPIS; KUMAR, 1995a; TENG, 1999; NOACK; ROTTA, 2009; VALEJO et al., 2014a; VALEJO; VALVERDEREBAZA; LOPES, 2014a). Furthermore, owing to its flexibility, the multilevel method has been applied to many classic problems, including network coloring (WALSHAW, 2001a),

1 We refer as large-scale networks with tens to few hundreds of thousands vertices and edges. We do not address massive networks with millions or even billions of vertices and edges, which must be processed with parallel, distributed or on-demand computing strategies. 
traveling salesman (WALSHAW; EVERETT, 2002), network drawing (ARLEO et al., 2018), network partitioning (LASALLE; KARYPIS, 2015) and computation of centrality measures (CHERNOSKUTOV; INEICHEN; BEKAS, 2015).

Despite their potential and the interest raised within the scientific community, existing multilevel approaches are not directly applicable to bipartite networks and, to the best of our knowledge, had not been considered in this context before, opening a large space for scientific exploration. This gap has motivated this research project, which investigated multilevel methods applicable to bipartite networks.

\subsection{Hypothesis}

Standard multilevel strategies do not consider vertex types, whereas the layers in a bipartite network usually represent distinct types of entities that must be handled separately. For the sake of illustration, suppose a document collection modeled as a document-word bipartite network. For a start, collapsing vertices that represent words with vertices that represent documents (i.e., matching vertices in different layers) would not be meaningful here and in most application scenarios. Moreover, as the number of words is typically much higher, coarsening the word layer may suffice to reduce the asymptotic convergence of a high-cost target algorithm. Furthermore, collapsing vertices of different types (e.g. word and author, document and word or user and location) will introduce a new hybrid entity which is not present in the original network, modifying its bipartite structure. Based on these assumptions, the first hypothesis is:

Hypothesis 1: An effective generalization (in terms of solution quality and computational cost) of the multilevel method to handle bipartite networks should consider the distinct vertex types and process the layers separately.

The first hypothesis encompasses the general use of the multilevel method in a bipartite structure. However, choosing a suitable matching strategy is a key component of an effective generalization of the multilevel method, since it is responsible for selecting the vertices to be collapsed. Consequently, the matching will interfere in the quality and bias of the coarsened representation. Therefore, the next hypotheses will address the matching process.

According to (FORTUNATO, 2010) and (NEWMAN, 2010) unipartite networks are commonly derived from bipartite structures. For example, scientific collaboration networks, in which two authors (vertices) are connected (edges) if both co-authored a paper, can be obtained from author-paper networks, in which a paper is associated with its authors. From this perspective, a bipartite network can be projected onto one of its vertex types, known as one-mode projection, so that vertices of the same type are connected with each other according to their original connection patterns (ZWEIG; KAUFMANN, 2011), i.e. vertices are connected if they share at least one common neighbor in the original bipartite representation. One-mode projection 
is, therefore, used as a useful view of information relative to bipartite networks. Since most algorithms and measures in network analysis consider unipartite networks, it is often practical to analyze a one-mode projection of a bipartite network (ZHOU et al., 2007; SETH; ZHANG, 2008; XU; WANG; GU, 2014; LINDELAUF; BORM; HAMERS, 2012). This strategy notably increases the range of analysis options available, as classic and already established proposals in the literature can be applied. This concept leads to the second research hypothesis:

Hypothesis 2: The one-mode projection allows classic coarsening algorithms to be employed in bipartite networks with no significant loss in solution quality and without impairing the scalability of the multilevel process.

Although employing one-mode projections has been successful and proved accurate in many contexts, most notably in community detection, there are problems derived from the network transformation, e.g. connectivity may be artificially inflated with the introduction of fully connected sub-graphs; the one-mode projection may hide latent features from the bipartite structures (KITSAK; KRIOUKOV, 2011; KITSAK; PAPADOPOULOS; KRIOUKOV, 2017); and there are many one-mode projection algorithms (BANERJEE; JENAMANI; PRATIHAR, 2017a; STRAM; REUSS; ALTHOFF, 2017; STRAM; REUSS; ALTHOFF, 2018; BANERJEE; JENAMANI; PRATIHAR, 2017b), consequently, selecting the best one can be a critical issue. Therefore, it can be more effective and efficient to perform mining tasks directly on the bipartite network, since it naturally captures the behavior of the observed system. Moreover, avoiding the one-mode projection can save processing effort and time.

The useful concept of $h$-hop neighborhood is considered in classical algorithms, such as PageRank, and in analytical tasks, e.g. in social network analysis $h$-hop neighborhoods are considered to identify structural holes or social circles, finding cliques or in link prediction (LIU; LI, 2018). Formally, the $h$-hop neighborhood of a vertex is defined as the set of vertices reachable from it by a path of length $h$. In bipartite networks vertices in the same layer (thus unconnected) can be accessed from each other through even-sized hop neighborhoods. Specifically, a local neighborhood composed by vertices of the same type can be reached with $h=2$, which yields notable computational savings, since a 2-hop neighborhood of a vertex can be reached in near-linear time (LIU; LI, 2018). The previous assumption leads to the third hypothesis:

Hypothesis 3: Restricting the matching to consider only 2-hop neighborhoods suffices to reflect the connection patterns of a bipartite network and can yield a cost-effective matching implementation.

The third hypothesis suggests a more robust solution in terms of solution quality and runtime. However, this assumption does not invalidate the potential benefits of the second hypothesis, which allows reusing classic and already established matching algorithms with good solution quality, it merely places them in a different perspective. 


\subsection{Objectives}

The previous hypotheses are formulated in a broad sense, i.e. with no specific application domain in mind. Therefore, the main objective of this thesis is to generalize the multilevel method to bipartite networks and investigate solutions capable of handling classic machine learning problems defined in this context. Consequently, this research provides a theoretical framework (or a meta-heuristic ${ }^{2}$ ) and useful resources for developing novel solutions targeted at particular applications modeled as bipartite networks. Accordingly, the following specific objectives were defined:

1. Investigation and development of a general-purpose multilevel method capable of handling bipartite networks.

2. Development of coarsening strategies that allow reusing classical coarsening algorithms in bipartite networks.

3. Development of contraction strategies directly applicable to bipartite networks, considering their connection patterns and concept of neighborhood.

4. Application and evaluation of the theoretical framework and algorithms developed to propose solutions capable of handling classic machine learning problems.

Besides the aforementioned objectives, the framework must also be flexible to be customized and adapted to different tasks. To achieve this goal, it is essential a previous background, specifically, a theoretical insight of the method, which links its key concepts into a logical structure independent of a particular application domain. However, we could not find a representative study to fit as a background to guide this research. Therefore, successively attaining the main goal demands the auxiliary objective summarized as follows:

5. Conduct a systematic mapping of the research contributions focused on the multilevel methods in complex networks, and provide an application-independent theoretical organization of the multilevel method and an account of the diverse problem domains to which multilevel optimization has been applied.

The development and performance comparison of machine learning algorithms often rely on an empirical investigation conducted over a representative set of benchmark networks, so that results are amenable to validation and statistical analysis of the observed differences in performance. That is, benchmarking is a rigorous strategy to contrast the strengths and weaknesses of multiple algorithms, to demonstrate their methodological capabilities, to guide the selection of an appropriate algorithm for a specific problem or simply to support the prototyping

2 Although an exact definition has been elusive, heuristics are defined as problem-dependent techniques and meta-heuristics are defined as high-level problem-independent techniques and can be used as a black box and applied to different problems with relatively few modifications (PAREJO et al., 2012). 
(or pre-production) development phase. However, the availability of benchmarking tools to assess solutions on bipartite networks is rather limited. Existing benchmarking tools in this context are either not accessible to researchers or lack proper documentation. Furthermore, the diversity of topological features characteristic of the real-world bipartite structure is not matched by established benchmarking tools, mainly when the focus is to synthesize large-scale bipartite networks. Therefore, to support the early development stages of the proposed algorithms and, later, accuracy assessment and comparison of alternative solutions, one auxiliary objective is summarized as follows:

6. Develop a benchmarking tool to generate large-scale bipartite network models that mimic a wide range of features from real-world networks, including community structures.

\subsection{Thesis Organization}

The thesis is organized as a collection of papers either published or currently under evaluation. I.e., the papers or submitted manuscripts that summarize the contributions of this research are presented as chapters (namely Chapters 2 to 7). The text, figures, algorithms and tables are based on the original papers with no copyediting and typesetting. It was required the standardization of terms, concepts and terminology to simplify communication between chapters. Furthermore, extra and supplemental materials were included in the chapters. Each chapter is self-contained and supplies the reader with the necessary background to understand the investigated research issue (e.g., definitions and notation). Although the reader may feel free to read these chapters in any order, the sequence of chapters reflects a hierarchical connection between the scientific advances achieved during this research. The remaining of this section summarizes the contents of each chapter.

\subsubsection{Chapter 2}

Title: A critical survey of the multilevel method in complex networks

This chapter is based on a manuscript submitted to the ACM Computing Surveys (CSUR) journal. Despite the success of multilevel optimization in a multitude of application problems, we could not find a representative survey of the state-of-the-art, or descriptions of the method in terms of a general theoretical framework independently of a particular application domain. To fill this gap, this chapter provides an extensive survey of the literature that contemplates a systematic overview of the current state-of-the-art, a discussion of the historical evolution of multilevel optimization in complex networks and current challenges, and a formal theoretical framework of the multilevel optimization method. 
Important decisions concerning this research were based on the findings of this systematic mapping. The primary studies inspired the selected research gaps. The theoretical framework assisted the development process itself, the planning of functions, architecture, and design of the software. The taxonomy supported the search for appropriate strategies for algorithm development. The contributions of this paper are summarized as:

- It presents a systematic mapping of the research contributions in the field, covering: 1) a description of its historical evolution; 2) a panorama of the current state-of-the-art; 3) an account of the diverse problem domains to which multilevel optimization has been applied; 4) a survey of relevant algorithms; and 5) a discussion of current research gaps and trends.

- It introduces a theoretical framework of the multilevel method, which: 1) provides a conceptual description of the method's phases while linking the key concepts into a logical structure; 2) allows establishing a taxonomy of the coarsening algorithms; and 3) enables a formal description of existing multilevel refinement algorithms.

\subsubsection{Chapter 3}

Title: A benchmarking tool for the generation of bipartite network models with overlapping communities

This chapter is based on a manuscript accepted for publication in the Knowledge and Information Systems (KAIS) journal (VALEJO et al., 2018a). Accurate assessment and comparison of alternative solutions are typically approached by benchmarking the target algorithm(s) on a set of diverse networks that exhibit a broad range of controlled features, ensuring the assessment contemplates multiple representative properties. Tools have been developed to synthesize bipartite networks, but none of the previous solutions address the issue of generating large-scale networks with non-overlapping and overlapping community structures. This chapter was motivated by this gap and introduces the BNOC tool, which allows synthesizing large-scale bipartite networks that mimic a wide range of features from real networks.

All algorithms and solutions developed in this research used BNOC, specifically, in the implementation and production phases and in the empirical analysis of the proposed algorithms. Therefore, the study establishes an important contribution to attain the research objectives. The article is not explicitly cited in the subsequent articles since it has been under review since 2017 and accepted in 2019. In summary, the contributions of this paper are:

- A novel benchmarking tool, called BNOC, to synthesize large bipartite networks with distinct community patterns in terms of number, size, balance, edge distribution intraand inter-communities, degree of overlapping and cohesion, and degree of noise in the connection patterns. 
- An outline of the generic principles underlying a generalization of BNOC to synthesize $k$-partite and heterogeneous networks.

\subsubsection{Chapter 4}

Title: Community detection in bipartite network: a modified coarsening approach

The chapter is based on the paper published in the Communications in Computer and Information Science book series (CCIS) (VALEJO et al., 2018a), which is an extended version of the paper presented in The International Symposium on Information Management and Big Data (SIMBig), 2017, One-mode projection-based multilevel approach for community detection in bipartite networks (VALEJO et al., 2017):

The chapter introduces a multilevel method based on one-mode projection to allow applying already established coarsening algorithms to bipartite networks. The article was selected among the best papers and invited to an extension. The main contributions of this article are:

- A novel multilevel method based on one-mode projection that allows executing traditional coarsening methods in bipartite networks.

- Guidelines to generalize the multilevel method to the community detection task.

\subsubsection{Chapter 5}

Title: Multilevel Approach for combinatorial optimization in bipartite networks

The chapter is based in the paper published in the Knowledge-based Systems (KNOSYS) jorunal (VALEJO et al., 2018b) and summarizes the most relevant contribution of this thesis. It introduces a multilevel method for bipartite networks and describes an implementation of a general multilevel framework applicable to a variety of problems. The contributions are as following:

- A novel multilevel optimization method applicable to problems modeled as bipartite networks.

- Two novel efficient matching algorithms, as well as novel contracting and uncoarsening algorithms.

- A comprehensive experimental evaluation on real and synthetic data. 


\subsubsection{Chapter 6}

Title: A coarsening method for labeled and unlabelled bipartite network via weight-constrained label propagation

The chapter is based on a manuscript submitted to the Knowledge-based Systems (KNOSYS) journal. The algorithms proposed in the previous chapters, despite their great potential, face theoretical and practical limitations. This chapter introduces a near-linear coarsening strategy based on weight-constrained label propagation. The strategy collapses groups of vertices, rather than vertex pairs and can yield faster network reduction rates. Moreover, users can specify the desired size of the coarsest network as the coarsening parameter and can control super-vertex weights. In summary, the contributions of this article are:

- A near linear ${ }^{3}$ matching strategy based on weight-constrained label propagation to address coarsening of bipartite networks. In a bipartite context, it is the first to allow collapsing groups of vertices, rather than vertex pairs.

- Two coarsening strategies that yield greater network reduction factors (on the order of $60 \%-90 \%$ ) at each level. The reduction rate can be specified either informing the number of coarsening levels or, alternatively, informing the desired size (number of vertices) of the coarsest network.

- A comprehensive experimental evaluation of the proposed strategy on real and synthetic bipartite networks that demonstrates its scalability and solution quality. It is employed to optimize a high-cost community detection algorithm, besides it can be employed directly as a community detection strategy.

- A discussion on the underlying applicability in the context of network visualization, illustrating its use in connection with network drawings obtained with force-directed algorithms.

\subsubsection{Conclusion}

The Chapter summarizes the main findings from the research carried out in this thesis, presents open problems and summarizes directions for future work.

$3 f: \mathbb{N} \rightarrow \mathbb{N}$ is near-linear if $f(n) \in \mathscr{O}\left(n \log ^{\mathscr{O}(1)} n\right)$ or $\mathscr{O}\left(n^{1+\mathscr{O}(1)}\right)$, i.e., if it is linear with respect to all the highest order derivatives 

CHAPTER

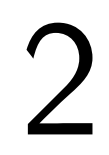

\section{A CRITICAL SURVEY OF THE MULTILEVEL METHOD IN COMPLEX NETWORKS}

Multilevel optimization aims at reducing the cost of executing a target network-based algorithm by exploiting coarsened, i.e. reduced or simplified versions of the network. There is a growing interest in multilevel algorithms in networked systems, mostly motivated by the urge for solutions capable of handling large-scale networks. Despite the success of multilevel optimization in a multitude of application problems, we could not find a representative survey of the state-ofthe-art, or descriptions of the method in terms of a general theoretical framework independent of a particular application domain. In this paper we make an effort to fill this gap, with an extensive survey of the literature that contemplates a systematic overview of the current state-of-the-art, a discussion of the historical evolution of multilevel optimization in complex networks and current challenges, and a formal theoretical framework of the multilevel optimization method. We hope this survey provides a useful resource for learning about multilevel strategies, as well as for advanced research and the development of novel application-oriented solutions.

\subsection{Introduction}

Complex networks are graphs with non-trivial topological properties that model interactions between elements in real-world systems. Such systems occur in diverse fields, from the biological to the social and technological domains, and complexity results naturally from the number of interacting elements and the dynamics of interactions. Network analysis comprises a range of methods to characterize, analyze, interpret, and simulate such complex networks (ZENG et al., 2017). Computationally expensive machine learning problems defined on networks, such as partitioning or classification, can be posed as finding a minimum (or maximum) cost, wherein the number of possible states is finite and usually exponential. Many such problems, e.g. finding a solution to clique, matching, vertex cover, community structure, traveling salesman or 
graph coloring problems (ASRATIAN; DENLEY; HäGGKVIST, 1998), have been proven to be $\mathscr{N} \mathscr{P}$-complete or $\mathscr{N} \mathscr{P}$-hard, for which the challenge of finding scalable solutions remains.

The multilevel method has been introduced in the 1990s as a practical strategy to solve combinatorial optimization problems defined in large-scale networks. The method comprises three phases, namely (i) network coarsening, (ii) solution finding, and (iii) network uncoarsening. The underlying rationale is to iteratively coarsen an initial network into a hierarchy of gradually smaller networks; solve the target problem in the coarsest network, and then successively project back the solution, possibly refining it, over the inverse sequence of coarsened networks, until a solution relative to the original network is obtained.

The method raised considerable interest and many papers have since been published analyzing specific aspects of multilevel optimization, or addressing particular applications. Illustrative examples include an empirical analysis of multilevel graph partitioning problems (KARYPIS; KUMAR, 1995a); a comparison of multiple coarsening schemes for multilevel graph partitioning (TENG, 1999); multilevel optimization developments to boost the performance of several combinatorial algorithms (WALSHAW, 2004; WALSHAW, 2008b); an effort to establish a parallel between coarsening, sampling, and smoothing in multi-grid and multilevel methods (CHEVALIER; SAFRO, 2009). In a broad study of graph summarization strategies covering from aggregation to bit-compression and simplification or filtering strategies the multilevel method is discussed as an application of graph summarization (LIU et al., 2018).

Most contributions from the literature address applications to a particular problem; or describe algorithms aimed at improving a specific stage, e.g. the matching algorithm required in the coarsening phase. The potential applicability of the method is huge, but relevant conceptual research and design questions remain open. Important gaps include: 1) The lack of a critical organization of the many coarsening approaches and associated matching algorithms, as well as of objective assessments of their relative advantages and limitations; 2) The lack of a comprehensive description of the historical and recent developments in multilevel algorithms, contemplating the current state-of-the-art and open research questions; 3) The lack of an application-independent theoretical framework, as most theoretical discussions target the network partitioning problem.

We address these gaps presenting a systematic survey of the literature on multilevel optimization methods applied to complex networks. This survey brings three major contributions:

A systematic mapping of the research contributions in the field: We conducted a broad study of the literature available in five scientific knowledge bases in order to identify the key contributions. Based on a meta-analysis of the papers retrieved, our survey covers: 1) a description of the historical evolution of the field; 2) a panorama of the current state-ofthe-art; 3 ) an account of the diverse problem domains to which multilevel optimization has been applied; 4) a discussion of current research gaps and trends. As such, this survey provides a contextualized reference to material produced thus far and a starting point to 
guide further studies. The meta-data relative to the papers surveyed is made available to the research community as supplemental material ${ }^{1}$.

A theoretical framework of the multilevel method: Considering our experience and the findings from the literature, we introduce a general framework of the multilevel method. This framework has both theoretical and practical implications: 1) it provides a conceptual description of the method's phases while linking the key concepts into a logical structure; 2) it allows establishing a taxonomy of the coarsening algorithms; and 3) it enables a formal description of existing multilevel refinement algorithms. Our discussion covers illustrative examples of the different multilevel phases and their interrelations, pointing to relevant papers from the literature.

Case studies: Employing the theoretical framework as guide, we present case studies illustrating how to address representative application problems from a multilevel perspective. This is an important resource and reference material that can inspire novel developments and usages of the method.

The remainder of this paper is organized as follows: in Section 2.2 we describe the protocol adopted in our systematic mapping of the literature and present a global view of the contributions found. In Section 2.3 we introduce the concepts and definitions required to understanding the multilevel algorithms in the context of complex networks. The general multilevel method framework is introduced in Section 2.4. The subsequent Sections 2.5 and 2.6 details theoretical overviews of the coarsening and the uncoarsening and cover a critical analysis of the multilevel method and applications. Section 2.7 we describe a few classical and representative applications addressed from a multilevel perspective. In Section 2.8 we summarize our findings and discuss current trends and promising research directions. A Glossary of terms and notation is given in 2.A and supplemental information relative to algorithms reviewed in the survey is depicted in 2.B.

\subsection{A panorama of research contributions on multilevel method}

We conducted a systematic mapping of the literature on multilevel methods following the methodology by Kitchenham et al. (2010), which comprises the steps of planning, execution and reporting research activities. In the planning step consists the research questions and the general search protocol are defined, as well as the inclusion criteria and which information will be extracted from the retrieved documents. The execution step comprises the collection and identification of the materials which meet the inclusion criteria. Documents are selected,

1 The material will be made available at $<$ https://github.com/alanvalejo/multilevel-review $>$ immediately after paper acceptance. 
synthesized and relevant information is organized. Finally, the material is scrutinized to produce the research report.

Unlike systematic reviews, which are aimed at producing an in-depth analysis of a limited number of primary studies (PETERSEN et al., 2008), systematic mappings seek to cover a broad range of materials in less detail. Our major goal was to identify the most relevant contributions in multilevel optimization methods in the context of complex networks, contemplating the problems and application domains addressed by these methods, the algorithms employed in the multilevel phases and their relative advantages and limitations. Following the previous three-step protocol we: (i) formulated the research question; (ii) specified the search strategy; and (iii) defined the criteria to establish publication acceptance.

We formulated the following research question: Which are the representative research activities in multilevel optimization in complex networks, including applications? Table 1 lists the relevant search keywords identified, plus variations and synonyms. Our previous knowledge of the topic has driven the choice of search strings, which is a potential source of bias.

Table 1 - Keywords, synonyms and related terms used to search articles.

\begin{tabular}{ll} 
Keyword & Synonyms and related terms \\
\hline multilevel & $\begin{array}{l}\text { multi-level, multilevel paradigm, multilevel scheme, } \\
\text { multilevel approach, multilevel optimization, multilevel method }\end{array}$ \\
coarsening & coarsen, contraction, simplification, reduction \\
complex network & graph, hypergraph
\end{tabular}

Source: Research data.

We searched five digital libraries (again, this choice is a potential source of bias), namely ACM Digital Library ${ }^{2}$; IEEE Xplore ${ }^{3}$; Science Direct ${ }^{4}$; Web of Science ${ }^{5}$; and Scopus ${ }^{6}$. Sources such as Google Scholar that index third party articles have not been considered. We used Mendeley ${ }^{7}$ for information selection and extraction and compiled a BibTex ${ }^{8}$ list of references into the Jabref ${ }^{9}$ reference manager. Whenever supported by the search engine, the following search expression was applied to the fields title, abstract and keywords, with settings adjusted to search for articles in English within the fields of computer science, mathematics and physics:

title-abs-key (multilevel or multi-level or coarsening or coarsen or "graph simplification" or "network simplification") and title-abs-key( graph or hypergraph or

\footnotetext{
$<\mathrm{http}: / / \mathrm{dl}$.acm.org $>$

$<\mathrm{http}: / /$ ieeexplore.ieee.org/Xplore $>$

$<$ http://www.sciencedirect.com>

$<$ https://apps.webofknowledge.com>

$<\mathrm{http}: / /$ www.scopus.com>

$<\mathrm{https}: / /$ www.mendeley.com/>

$<$ http://www.bibtex.org/>

$<$ http://www.jabref.org/>
} 
“complex network”) limit-to(area, “computer science” or physics or mathematics) and limit-to(language, English)

A total of 781 documents published between 1980 and December 2018 have been retrieved, as summarized in Table 2. Documents meeting any of the following criteria were excluded: (i) is a duplicate; (ii) is a condensed version of a more complete document (preserved); (iii) hosting service is inaccessible or access-restricted; (iv) is not available in full, or is a summary document (poster, abstract, editorial, abstract of thesis or dissertation); (v) is not written in English; (vi) has been retracted; or (vii) is unrelated with the topic of multilevel optimization in complex networks.

Table 2 - Number of papers retrieved from each database.

\begin{tabular}{lc} 
Dataset & Articles \\
\hline IEEE Xplore & 147 \\
Science Direct & 45 \\
Web of Science & 285 \\
ACM & 24 \\
Scopus & 270 \\
\hline Total & 781 \\
\multicolumn{2}{c}{ Source: Research data. }
\end{tabular}

A total of 372 papers have been identified as duplicates and 175 have been rejected following an exclusion criteria, most often for being unrelated with the topic. The remaining 234 papers have been accepted and we extracted the following information from each:

1. Metadata: abstract, title, author, publication year, publication vehicle.

2. Application domain: the problem or applications addressed in the paper.

3. Whether it introduces an algorithm for a phase of the multilevel method, reports an application, or both;

4. A description of the algorithm introduced or applied;

5. The claimed advantages of the algorithm;

6. The reported disadvantages and limitations;

7. The availability of the proposed algorithm (as source code);

8. The multilevel phase addressed by the algorithm, i.e., coarsening, solution finding, or uncoarsening and refinement.

The data collected ${ }^{10}$ has been inspected in order to summarize the most relevant contributions, establish connections between multiple research branches and identify current research $\overline{10}$ available as supplementary material to this paper. 
trends. The yearly distribution of the publications, shown in Fig. 1, reveals a growing number of papers, with a peak in 2018, reinforcing our perception on the current relevance of the multilevel method.

The origins of multilevel methods date back to the concept of graph contraction, introduced as a general method for solving several graph problems efficiently, as reviewed by Babai (1977) and Gabow, Galil and Spencer (1989). The early concept generalized as later attempts to handle graph partitioning evolved, eventually originating the term "multilevel method", also referred as "multilevel strategy", "multilevel approach" or "multilevel optimization". Although in this work we focus specifically on its usage in the context of machine learning in complex networks, the method is employed in multiple contexts.

Attention to multilevel methods was largely motivated by the encouraging results obtained in applying multi-grid techniques to reduce the search space of complex problems (TENG, 1999; BRANDT, 1988). Popularity boosted after publication of important studies by Cong and Smith (1993), Mansour et al. (1993), Ponnusamy et al. (1994), Barnard and Simon (1994) and Barnard (1995), these last two under the influence of Eppstein (1992) and Mansour and Fox (1992). Several other publications contributed to consolidate the multilevel theory on complex networks (HENDRICKSON; LELAND, 1995; KARYPIS; KUMAR, 1995a; KARYPIS; KUMAR, 1995b).

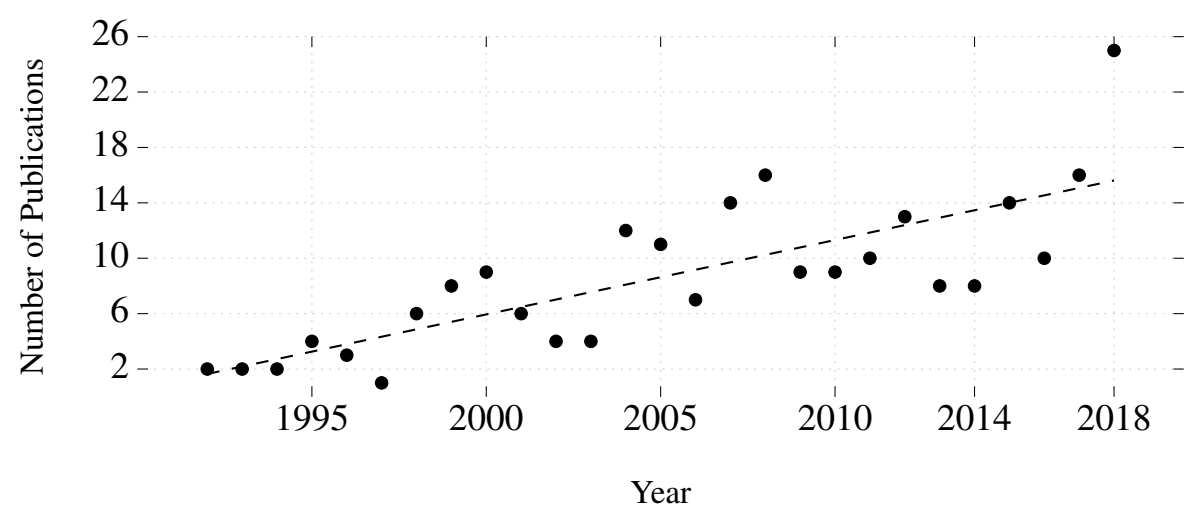

Figure 1 - Number of publications per year, from 1992 to December 2018.

Source: Elaborated by the author.

The nature and diversity of the publication venues mirror the relevance of the topic. We identified 92 journal papers, 89 conference papers and 53 papers in symposiums and workshops. The vehicles include reputed journals such as Information Science (Impact Factor 4.832), Pattern Recognition (IF 4.582), Knowledge-Based Systems (IF 4.529) and IEEE Transactions on Parallel and Distributed Systems (IF 4.181), and high-quality conferences such as the International Conference on Knowledge Discovery in Data Mining (SIGKDD), ACM International Conference on Information and Knowledge Management (CIKM), International Joint Conference on Artificial Intelligence (IJCAI), European Conference on Parallel Computing (Euro-Par) and International Conference on Computational Science (ICCS). 
Table 3 shows the top 5 cited papers according to Google Scholar, where the "cited by" metric can gauge their visibility and impact. The paper by Blondel et al. (2008) introduced Louvain, the first highly accurate multilevel method for community detection on large networks; the papers by Karypis and collaborators (KARYPIS; KUMAR, 1998a; KARYPIS; KUMAR, 1998b; KARYPIS et al., 1997) introduced the popular algorithms Random Matching (RM) and Heavy-Edge Matching (HEM) for multilevel coarsening. Hendrickson and Leland (1995) introduced a multilevel partitioning software package into a network partitioning library (CHACO 2.0).

Table 3 - Top 5 cited papers (source: Google Scholar, accessed in 2019 January).

\begin{tabular}{|c|c|c|}
\hline Reference & Title & Cited By \\
\hline Blondel et al. (2008) & Fast unfolding of communities in large networks & 96862008 \\
\hline Karypis and Kumar (1998a) & A Fast and High Quality Multilevel Scheme for Partitioning Irregular Graphs & 53181998 \\
\hline Karypis and Kumar (1998b) & Multilevel k-way Partitioning Scheme for Irregular Graphs & 18441998 \\
\hline Karypis et al. (1997) & Multilevel hypergraph partitioning: Application in VLSI domain & 14831997 \\
\hline Hendrickson and Leland (1995) & A multilevel algorithm for partitioning graphs & 14141995 \\
\hline
\end{tabular}

Source: Research data.

Table 4 shows the top 5 cited papers over the last ten years (2009-2018), with influential contributions, e.g. by Noack and Rotta (2009), who reported an empirical comparison of existing coarsening and refinement heuristics based on modularity optimization; Sanders and Schulz (2011), who presented a multilevel network partitioning algorithm based on multi-grid linear solvers; LaSalle and Karypis (2013), who introduced and compared multiple approaches to parallelize the multilevel phases in network partitioning. Benlic and Hao (2011) introduced a multilevel approach which combines memetic and tabu search algorithms; Ron, Safro and Brandt (2011) generalized and improved the multiscale organization of networks with a measure that quantifies the "closeness" between a pair of vertices.

Table 4 - Top 5 cited papers in the last ten years (2008-2018) (source: Google Scholar, accessed in January 2019).

\begin{tabular}{llrr} 
Reference & Title & Cited By & Year \\
\hline Noack and Rotta (2009) & Multi-level algorithms for modularity clustering & 160 & 2009 \\
Sanders and Schulz (2011) & Engineering multilevel graph partitioning algorithms & 126 & 2011 \\
LaSalle and Karypis (2013) & Multi-threaded graph partitioning & 126 & 2013 \\
Benlic and Hao (2011) & A multilevel memetic approach for improving graph k-partitions & 81 & 2011 \\
Ron, Safro and Brandt (2011) & Relaxation-Based Coarsening and Multiscale Graph Organization & 79 & 2011
\end{tabular}

Source: Research data.

We also verified which application problems and network organizations are most frequent in the publications, as well as reported usages of concurrent programming paradigms. Fig. 2a shows the articles distribution regarding the network organization. Most consider simple weighted networks, also hypergraphs in the context of multilevel partitioning for VLSI circuit design in the 90's. Recent contributions consider problems defined in bipartite networks (VALEJO et 
al., 2017; VALEJO et al., 2018a; VALEJO et al., 2018b). Heterogeneous or general k-partite networks have been little explored (LIU et al., 2014).

Even though they reduce the computational cost of solving complex optimization problems, sequential multilevel algorithms, even of near-linear time complexity, are still unfeasible on networks with hundreds of millions, or billions vertices and edges. Handling massive networks requires distributed processing or parallel implementations ${ }^{11}$. Fig. 2 b shows the distribution of the articles regarding the usage of concurrent programming paradigms: around $37 \%$ resort to some type of parallelism as opposed to sequential implementations.

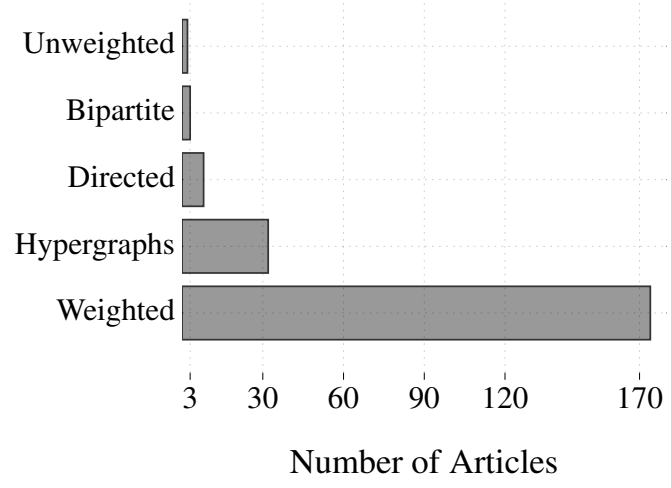

(a)

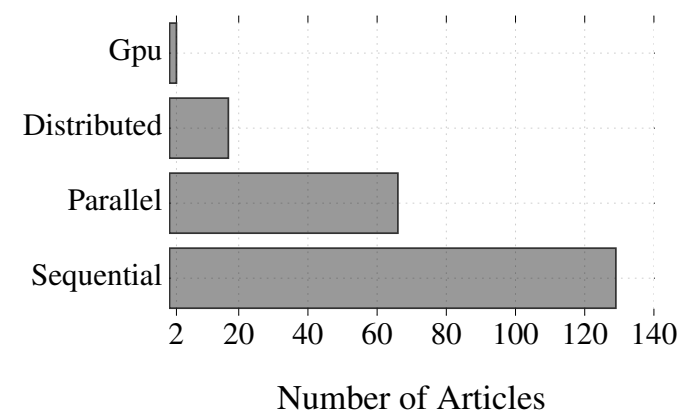

(b)

Figure 2 - Distribution of the papers selected, according to: (a) the network structure they consider; (b) the usage of concurrent or parallel paradigms versus sequential programming.

Source: Elaborated by the author.

As summarized in Table 5, the multilevel method has applications in diverse problem domains. Network partitioning (also known as community detection, or clustering) motivated most early research efforts and is currently a benchmark for multilevel methods. Other popular applications include network drawing and image segmentation. Image segmentation solutions often adopt a distinct terminology for the coarsening phase, referred to as aggregation, simplification or filtering. To a lesser extent we also identified applications in dimensionality reduction, graph contraction (and the related problems of size reduction and matrix approximation), classification, finding functional modules in biological networks, name disambiguation, graph coloring, traveling salesman problem, and document clustering. There are also applications in classical graph problems such as average shortest-path computation, matrix reordering, computation of centrality measures, text information retrieval, feature selection, and linear arrangement.

Walshaw and Everett (2002), Walshaw (2004), Walshaw (2008b) presented evidence on the potential of the multilevel optimization strategy for handling several complex problems, such as Vehicle Routing Problem (VRP), Travelling Salesman Problem (TSP), Graph Ordering, Graph Colouring, DNA Sequencing by Hybridization, Covering Design, Capacitated Multicommodity

11 Parallelism refers to a single process executing multiple computations in parallel; distributed solutions refer to concurrent process require coordinated work and communication. 
Table 5 - Relevant applications and related papers.

\begin{tabular}{|c|c|}
\hline Application & References \\
\hline \multirow[t]{4}{*}{ Partitioning } & (HENDRICKSON; LELAND, 1995), (KARYPIS; KUMAR, 1995a),(PELLEGRINI; ROMAN, 1996) \\
\hline & (WALSHAW; CROSS, 1998), (KOROŠEC; ŠILC; ROBIČ, 2003), (OLIVEIRA; SEOK, 2006) \\
\hline & (SCHUETZ; CAFLISCH, 2008a), (KARYPIS et al., 1997) \\
\hline & (NOACK; ROTTA, 2009), (VALEJO; VALVERDE-REBAZA; LOPES, 2014a), (LASALLE; KARYPIS, 2015) \\
\hline \multirow[t]{6}{*}{ Network drawing and visualization } & (WALSHAW, 2001a), (HACHUL; JÜNGER, 2005),(HUANG et al., 2007) \\
\hline & (ZHOU; HUANG, 2008), (GANSNER et al., 2011), (CRAWFORD; WALSHAW; SOPER, 2012) \\
\hline & (ALVISI, 2015), (TOOSI; NIKOLOV, 2016), (MEYERHENKE; NOLLENBURG; SCHULZ, 2018) \\
\hline & (ARLEO et al., 2018), (ABELLO; HAM; KRISHNAN, 2006), (Archambault; Munzner; Auber, 2008) \\
\hline & (BATAGELJ et al., 2011), (DIAS et al., 2017), (PERROT; AUBER, 2018) \\
\hline & $\begin{array}{l}\text { (MEYERHENKE; NÖLLENBURG; SCHULZ, 2015), (GALAN; MENGSHOEL, 2018),(Wong et al., 2009) } \\
\text { (FRISHMAN: TAL, 2007) }\end{array}$ \\
\hline \multirow{2}{*}{ Image segmentation } & $\begin{array}{l}\text { (FRISHMAN; TAL, 2007) } \\
\text { (LOMBAERT et al., 2005), (TOLLIVER; COLLINS; BAKER, 2007), (DUCOURNAU et al., 2009) }\end{array}$ \\
\hline & (KHOKHER; GHAFOOR; SIDDIQUI, 2012), (SIDDIQUI; GHAFOOR; KHOKHER, 2013), (MA et al., 2013) \\
\hline Dimensionality reduction & (SAKELLARIDI; FANG; SAAD, 2008), (FANG; SAKELLARIDI; SAAD, 2010), (VALEJO et al., 2018b) \\
\hline \multirow[t]{3}{*}{ Graph contraction } & (PONNUSAMY et al., 1994), (TENG, 1999), (ARENAS et al., 2007) \\
\hline & (DIAS et al., 2017), (HEUER; SCHLAG, 2017), (UBARU; SAAD, 2017) \\
\hline & (CHENG-GEN et al., 2008) \\
\hline \multirow[t]{2}{*}{ Classification and Graph coloring } & (WANG; ZHANG, 2007), (ZHANG; WANG, 2010), (WALSHAW, 2001b) \\
\hline & (WALSHAW, 2004) \\
\hline \multirow[t]{2}{*}{ Traveling salesman problem } & (WALSHAW, 2001c), (WALSHAW; EVERETT, 2002), (WALSHAW, 2004) \\
\hline & (WALSHAW, 2008b) \\
\hline \multirow[t]{2}{*}{ Finding functional modules } & (OLIVEIRA; SEOK, 2006), (OLIVEIRA; SEOK, 2007b), (OLIVEIRA; SEOK, 2007a) \\
\hline & (OLIVEIRA; SEOK, 2008) \\
\hline \multirow[t]{3}{*}{ Others } & (MAO; ZHANG, 2014), (OSEI-KUFFUOR; LI; SAAD, 2015), (CHERNOSKUTOV; INEICHEN; BEKAS, 2015) \\
\hline & (WALSHAW, 2008b), (SAFRO; RON; BRANDT, 2006), (ON; LEE, 2007) \\
\hline & (OLIVEIRA; SEOK, 2005), (FANG; SAAD, 2010), (JIAN-BIN; XIANG-JUN; HAN-TAO, 2004) \\
\hline
\end{tabular}

Network Design (CMND), Biomedical Feature Selection. An early study (BARNARD; SIMON, 1994) introduced a multilevel version of the recursive spectral bisection method involving unstructured mesh problems, for which a parallel implementation was later designed (BARNARD, 1995). In a recent paper, Valejo et al. (2018b) introduced a general framework to apply the multilevel method to problems modeled in bipartite networks, including dimension reduction, classification and network visualization.

Fig. 3 shows the co-authorship networks of Walshaw and Karypis, two highly influential authors in the field. Walshaw's co-authorship network, in Fig. 3a, is associated with 38 papers (18\% of the total) published from 1998-2012 and also includes the most connected author (Schulz, C.). The corresponding paper's timeline shown in Fig. 3a is highly informative of the field's evolution over the period (PELLEGRINI; ROMAN, 1996; WALSHAW; CROSS, 2000a; WALSHAW; CROSS, 2007; WALSHAW; CROSS, 2000b; WALSHAW, 2008b; WALSHAW, 2001a; WALSHAW, 2004). These papers address various applications, e.g mesh partitioning, graph coloring, the traveling salesman problem, graph drawing and graph partitioning. Fig. $3 \mathrm{c}$ details the co-authorship network of Karypis, who authored three of the top 5 most cited papers in the field, see the timeline in Fig. 3c. This component also includes authors of other highly influential papers (KARYPIS; KUMAR, 1996; KARYPIS et al., 1997; KARYPIS; KUMAR, 1998b; KARYPIS; KUMAR, 1998a; KARYPIS; KUMAR, 1999b; KARYPIS; KUMAR, 1999a; ABOU-RJEILI; KARYPIS, 2006; LASALLE; KARYPIS, 2013).

As a final observation, we highlight several public domain packages made available for 


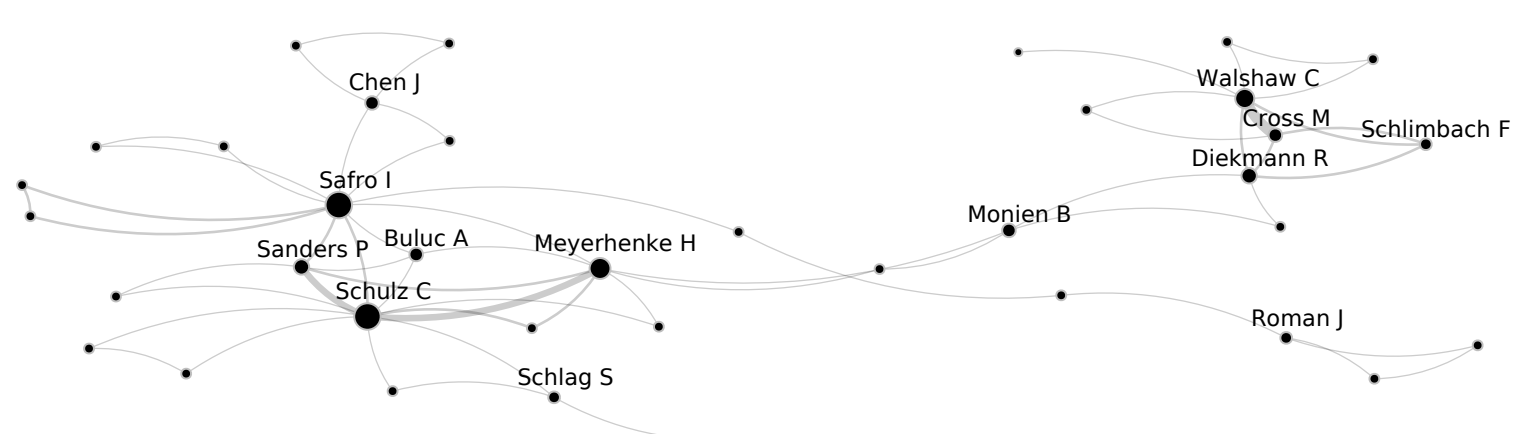

(a)

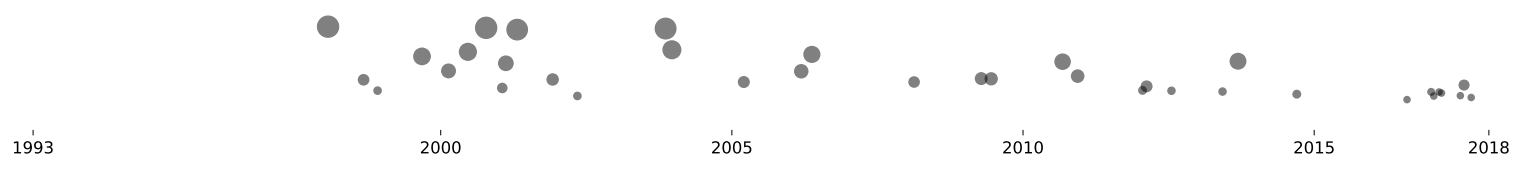

(b)

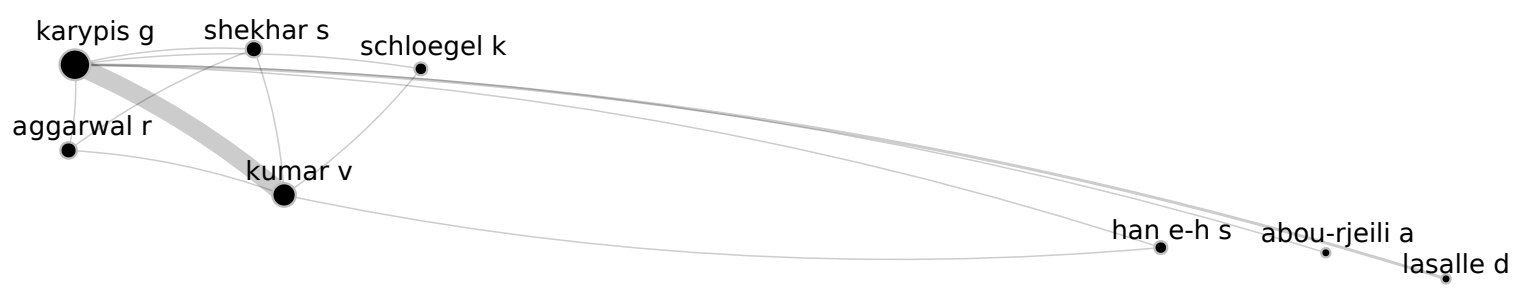

(c)

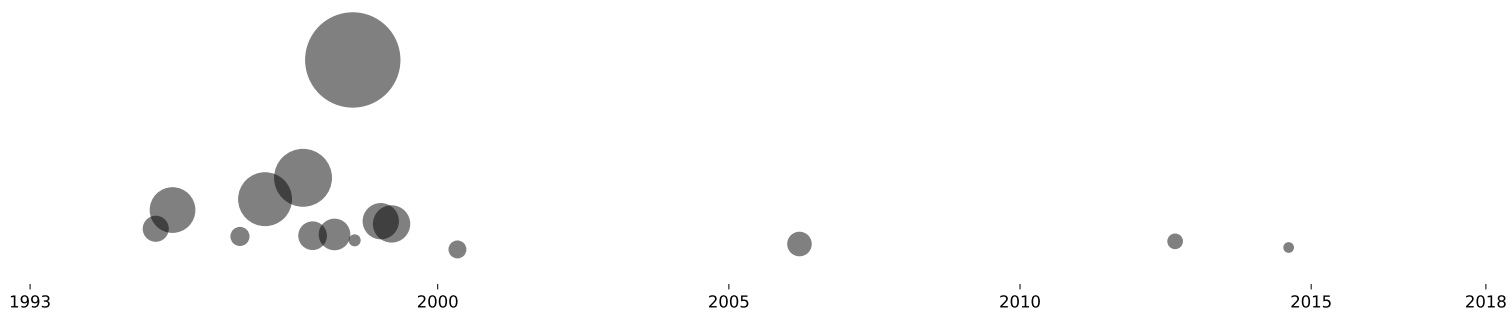

(d)

Figure 3 - Walshaw's and Karypis's co-authorship networks: line width maps the number of coauthorships, circle size maps the number of co-authored papers. Under each network a timeline of the publications is shown: each circle represents a paper with circle size proportional to the paper's citation count.

multilevel network partitioning, such as JOSTLE (WALSHAW; CROSS, 2000a), METIS (KARYPIS;

KUMAR, 1995b), CHACO (HENDRICKSON; LELAND, 1995), SCOTCH (PELLEGRINI; ROMAN, 1996), PT-SCOTCH (CHEVALIER; PELLEGRINI, 2006), OPM (VALEJO et al., 2017) and MOb (VALEJO et al., 2018b).

\subsection{Basic Concepts}

A unipartite (or simple) and undirected network is represented as $G=(V, E, \sigma, \omega)$, where $V=\left\{v_{1}, v_{2}, \ldots, v_{n}\right\}$ is the set of vertices, $E=\left\{e_{1}, e_{2}, \ldots, e_{m}\right\}$, is the set of edges connecting vertices, i.e. $E \subseteq V \times V$, such that $e_{i}=\{(u, v)=(v, u) \mid u, v \in V\}$ and $\omega=\left\{\omega_{1}, \omega_{2}, \ldots, \omega_{m}\right\}$ is 
the set of weights, i.e. $\omega: V \mathrm{x} V \rightarrow \mathbb{R}^{*}$, so that each $\omega_{i}$ is associated with a corresponding edge $e_{i}$. The total edge weight is equal to $\omega=\omega(E)=\sum_{i} \omega_{i}$. The weight of a vertex $v_{j}$ is denoted by $\sigma\left(v_{j}\right)$ with $\sigma: V \rightarrow \mathbb{R}^{*}$, and $\sigma(V)=\sum_{j} \sigma\left(v_{j}\right)$ is the total network vertex weight. Let $n=|V|$ and $m=|E|$ denote, respectively, the number of vertices and the number of edges, where operator "| |" stands for the cardinality of a set.

Two vertices $u$ and $v$ connected by at least one edge are called neighbors (or adjacent), otherwise $u$ and $v$ are non-neighbors (non-adjacent). The edge is said to be incident to its vertices. Two edges sharing a common vertex are adjacent, otherwise they are non-adjacent, or disjoint. An isolated vertex has no incident edges, and a leaf vertex has a single incident edge.

A bipartite network is represented as $B(V, E, \sigma, \omega)$, where $V$ is partitioned into two sets $V_{1}$ and $V_{2}$ so that $V_{1} \cap V_{2}=\emptyset . V_{1}=\left\{u_{1}, u_{2}, \ldots, u_{n_{1}}\right\}$ is a set of vertices, or layer, $V_{2}=\left\{v_{1}, v_{2}, \ldots, v_{n_{2}}\right\}$ is another layer and $E=\left\{e_{1}, e_{2}, \ldots, e_{k}\right\}$ is the set of edges connecting vertices in different layers, i.e. $\forall(u, v) \in E \Rightarrow u \in V_{1}, v \in V_{2}$ and $E \subseteq V_{1} \times V_{2}$. Similarly, $\omega=\left\{w_{1}, w_{2}, \ldots, w_{k}\right\}$ is the set of edge weights.

$\Gamma_{h}(v)$ denotes the $h$-hop neighborhood of a vertex $v$, formally defined as the set of vertices $\Gamma_{h}(v)=\{u \mid$ there is a path of length $h$ between $v$ and $u\}$. Thus, the 1-hop neighborhood of $v$, $\Gamma_{1}(v)$, is the set of vertices adjacent to $v$; the 2-hop neighborhood, $\Gamma_{2}(v)$, is the set of vertices 2-hops away from $v$, and so forth. The degree $d_{v}$ of a vertex $v$ is the number of neighbors of $v$; its strength $s_{v}=\sum_{u \in \Gamma_{1}(v)} \omega(v, u)$ is the total weight of its adjacent edges. The degree of a graph $G$ is $d_{G}=\sum_{u \in V} d_{u}$; similarly its strength is $s_{G}=\sum_{u \in V} s_{v}$. The few vertices (usually only [0.01-5]\% of the network) with the highest degree (or strength), called hubs in scale-free networks.

A strict clustering, or community structure, $C=\left\{C_{i}\right\}_{i=0}^{k-1}$ is a partition of $V$ into $k$ nonempty $\left(1 \leq\left|C_{i}\right|, \forall i\right)$ and disjoint $\left(i \neq j \Rightarrow V_{i} \cap V_{j}=\varnothing\right)$ subsets $C_{i} \in C$ such that $V=C=\bigcup C_{i}$, i.e. each vertex belongs to exactly one cluster. $C(u)$ returns the cluster $C_{i}$ that contains the vertex $u$. Let $C_{i}^{i n}$ be the sum of the weights of the edges ending in $C_{i}, C_{i}^{\text {out }}$ the sum of the weights of the edges that connect vertices in $C_{i}$ to vertices in other clusters. Let also $d_{u \rightarrow C_{i}}$ be the number of neighbors of $u$ that are in $C_{i}$. The number of edges connecting $C_{i}$ to $C_{j}$ is denoted by $d_{C_{i} \leftrightarrow C_{j}}$. The cluster $C_{i}$ with the vertex $u$ removed (or added) is denoted by $C_{i}-\{v\}$ (or $C_{j}+\{v\}$ ). A cluster $C_{i}$ formed by a single vertex is called a singleton. A vertex $u$ is a boundary vertex if it is connected with two or more communities, $u$ is a non-boundary vertex otherwise.

A network clique is a fully connected sub-network $C\left(U, E_{U}\right)$, in which $U \subset V$ and $E_{U} \subset E$ and every two distinct vertices are adjacent. A $k$-clique is a clique of cardinality $k$, e.g, a 2-clique is simply an edge. A similarity score $S(u, v)$ can be computed for a pair of vertices $u$ and $v . S(u, v)$ is in the range $[0,1] \subset \mathbb{R}$, in which 0 denotes lowest similarity and 1 denotes highest. A fundamental structural similarity function is given by the relative number of common neighbors: $S_{c n}(u, v)=|\Lambda(u, v)|, \Lambda(u, v)=\left\{\Gamma_{1}(u) \cap \Gamma_{1}(v)\right\}$ (LÜ; ZHOU, 2010). The local clustering coefficient of a vertex $v, c c(v)$, quantifies how close its neighbors are to being a clique, i.e., the ratio between the actual and the potential number of edges in the vertex 
neighborhood (WATTS; STROGATZ, 1998; NEWMAN, 2003).

\subsection{Multilevel method framework}

Multilevel optimization can be defined as a meta-heuristic aimed at reducing the cost of executing a target algorithm over a given network. The rationale is to execute the algorithm in a reduced version of the original network to obtain an initial solution, then project and refine this initial solution, over multiple iterations, up to the original network. The approach increases the practical scalability of complex algorithms, allowing them to be executed on large-scale networks. In this section we provide an overview of the multilevel method, with a focus on central definitions and on the general theoretical framework. Our description is grounded on the literature presented in the previous section, mainly the contributions by Karypis and Kumar (1998a) and Walshaw (2004).

The method, illustrated in Fig. 4, comprises the phases of coarsening (split into matching and contraction), solution finding (with the target algorithm), and uncoarsening (split into projection and refinement). In the coarsening phase the original network is successively reduced to obtain a sequence of coarsened networks up to a coarsest network; in the solution finding phase an initial solution is obtained executing the target algorithm in the coarsest network; in the uncoarsening phase the initial solution is successively projected back to the intermediate networks and refined, until obtaining the final solution in the original network.

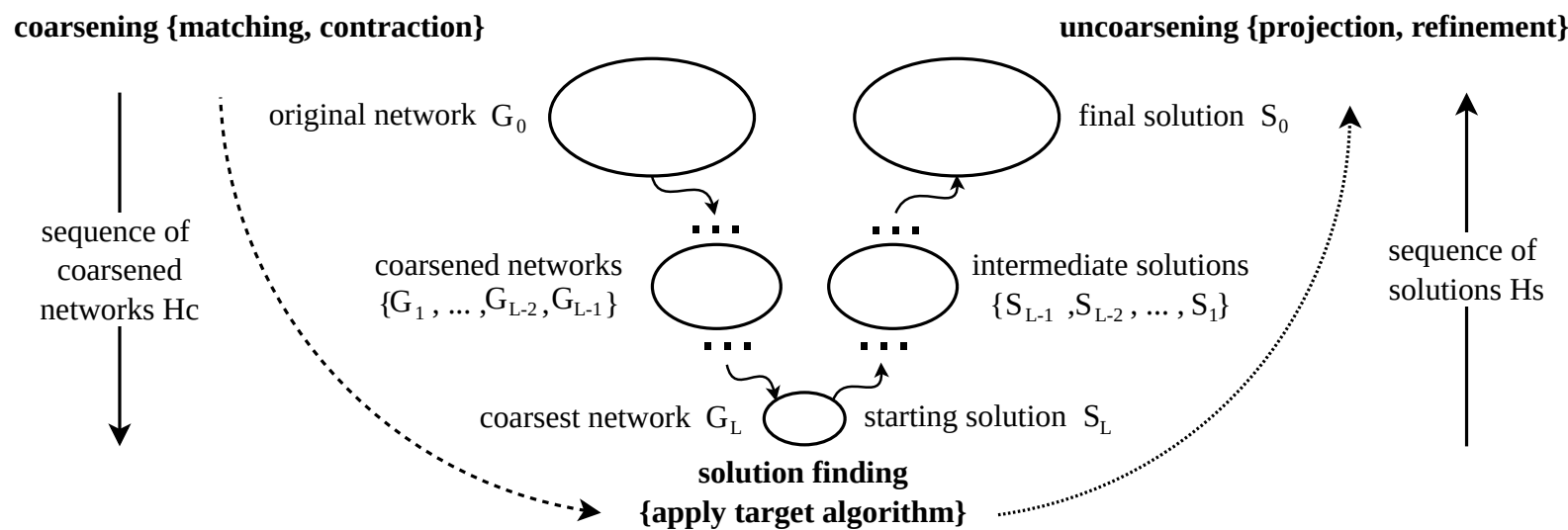

Figure 4 - Phases of a multilevel optimization process: coarsening, solution finding, and uncoarsening; and the entailed sequences of networks and solutions.

Let $G_{0}$ be the initial network in which the original problem is defined, and $G_{L}$ be the coarsest network obtained after $L$ coarsening steps (levels). Let $S_{L}$ be the initial solution found in $G_{L}$, and $S_{0}$ be the final (refined) solution found in $G_{0}$. Let also $H_{C}=\left\{G_{l}\right\}_{l=0}^{L}$ be the sequence of coarsened networks and $H_{S}=\left\{S_{L-l}\right\}_{l=0}^{L}$ be the sequence of solutions, where $l$ is the coarsening level.

Algorithm 1 describes the multilevel optimization (MO) framework, encompassing coarsening (lines 1-4), solution finding (line 6) and uncoarsening (lines 7-9). It takes as inputs 
the initial network $G_{0}=\left(V_{0}, E_{0}, \sigma_{0}, \omega_{0}\right)$, a desired number of coarsening levels $L$ and a reduction factor $\rho$. The network is progressively coarsened, level-by-level, by means of calls to a matching algorithm (line 2) and to a contracting algorithm (line 3), until it is reduced by the desired factor. The stopping criterion can be specified informing a target size of the coarsest network, or a target reduction rate. Alternatively, the algorithm may stop when an arbitrarily small rate of network reduction is achieved between successive coarsening levels. The matching algorithm is responsible for selecting, based on some criterion, the pairs or groups of vertices to be matched (line 2). Matched vertices (pairs or groups) are collapsed into super-vertices to form a coarsened network. The target algorithm is then executed in the coarsest network thus obtained, $G_{L}$, to compute an initial solution $S_{L}$ (line 6). Finally, in the uncoarsening phase, this solution is projected along the space of intermediate networks, creating a space of intermediate solutions $\left\{S_{L-l}\right\}_{l=1}^{L}$, up to the final solution $S_{0}$ in the initial network $G_{0}$ (lines 7-10); solutions are possibly refined at each level (line 9).

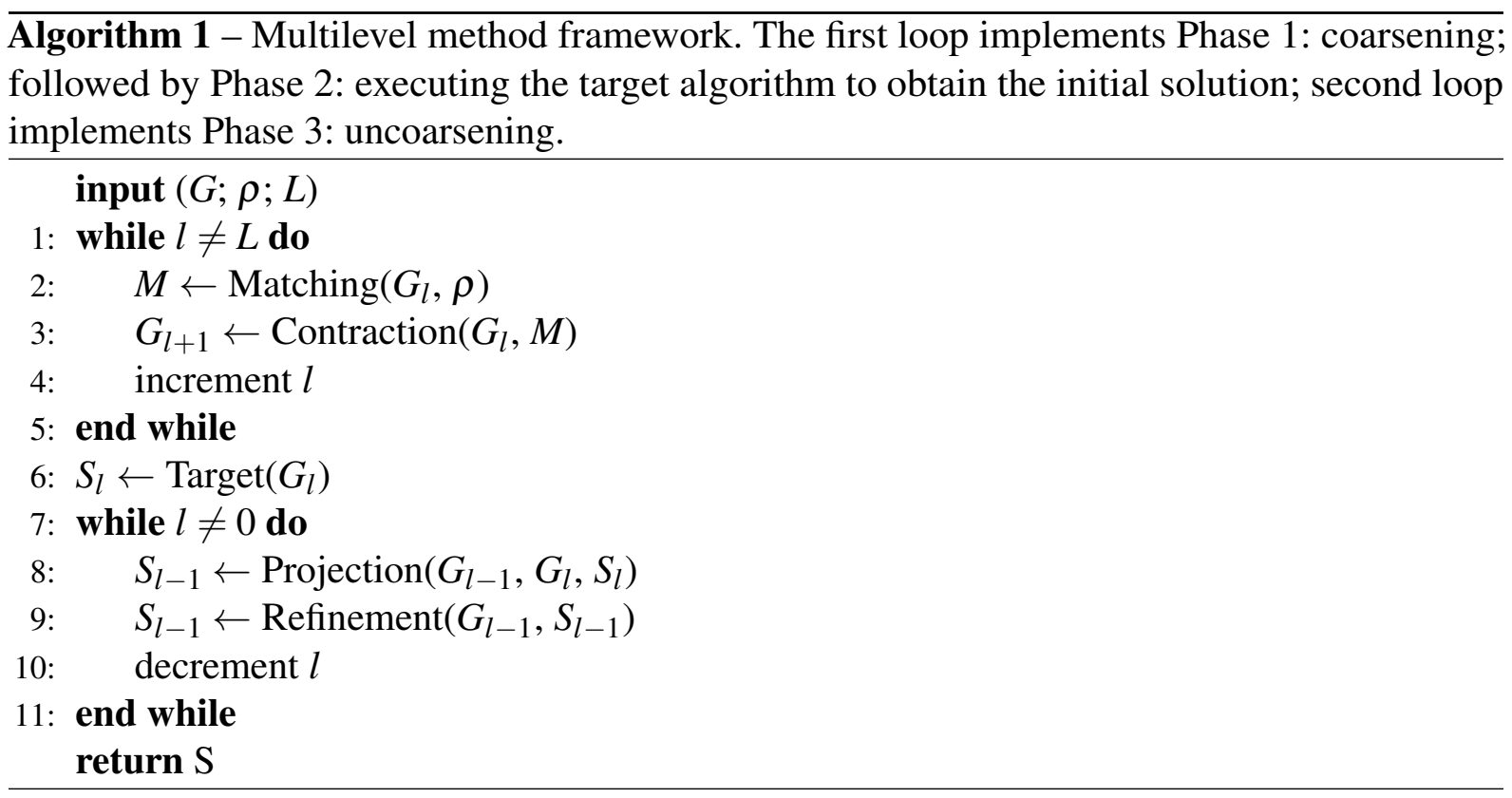

Let $S$ be the set of all possible solutions in the coarsest problem instance. Given an objective function $f: S \rightarrow \mathbb{R}$ (or $\mathbb{N}$ ) that assigns a cost to each solution in $S$, the aim is to find a solution $s \in S$ with optimal cost. For instance, in the traveling salesman problem $s$ is a path and $f(s)$ expresses its length, whereas in a community detection problem $s$ is a network partition and $f(s)$ denotes some measure of community quality. In certain scenarios the target algorithm is suppressed, as the coarsest network itself is the initial solution, e.g., it provides a simplified representation for visualization purposes, or its super-vertices correspond to the communities of a network partitioning.

Since, in principle, the coarsest network can be arbitrarily small, it becomes feasible to apply a computationally expensive algorithm to compute an initial solution to a problem. Let an idealized $\mathrm{MO}$ be such that at each coarsening level all vertices are matched in pairs, so that the size $|V|$ of each network is reduced by $50 \%(\rho=0.5)$. If $T_{X}^{l=0}$ is the cost of executing 
a target algorithm of linear complexity, i.e. $T_{X}=\mathscr{O}(n)$, in a network of size $|V|=n$, then the cost of computing an initial solution in the coarsest network is $T_{X}^{l=L}=T_{X}^{l=0} /\left(2^{L}\right)$, wherein $L$ is the final coarsening level. Notice that $l=1$ yields two networks of sizes $(n, n / 2), l=2$ yields $(n, n / 2, n / 4), l=3$ yields $(n, n / 2, n / 4, n / 8)$ and so on. Hence, the coarsest network will have size $n /\left(2^{L}\right)$ and therefore $T_{X}^{l=L}=T_{X}^{l=0} /\left(2^{L}\right)$. Thus, parameter $L$ may be set according to the cost deemed acceptable to compute the initial solution.

As an illustration, let us consider another scenario of two hypothetical target algorithms $X$ and $Y$ with complexity $T_{X}=\mathscr{O}(n)$ and $T_{Y}=\mathscr{O}\left(n^{2}\right)$, respectively. In a network with $n=100$ vertices, for instance, $T_{X}=\mathscr{O}(100), T_{Y}=\mathscr{O}(10,000)$. If one wishes to execute algorithm $Y$ on a network with $n=10,000$ vertices with runtime equivalent to algorithm $X$, the original network should be coarsened to a size $\sqrt{n}=100$, which assuming $\rho=0.5$ requires setting $L \approx 7$. In this case, $T_{X}=\mathscr{O}(n)=\mathscr{O}(10,000)$ and $T_{Y}^{L=7}=\mathscr{O}\left(n^{2}\right)=\mathscr{O}\left(100^{2}\right)=10,000$. Of course, algorithm $Y$ still has quadratic asymptotic complexity, but it can now be executed in time equivalent to the runtime of the linear algorithm in the original network. In general, the computational complexity of the target algorithm can guide parameter setting, e.g. in defining the expected size of the coarsest network.

Given this overview of the MO method, we present detailed theoretical descriptions of the phases of coarsening, in Section 2.5, and uncoarsening in Section 2.6. The solution finding phase is obviously application-dependent. Whereas popular applications have been mentioned in Section 2.2, this phase shall not be discussed any further in this text.

\subsection{Coarsening}

The coarsening phase outputs a sequence of progressively coarsened networks $G_{l}$ departing from the initial network $G_{0}$, i.e. a set of intermediate approximations of $G_{0}$ at multiple levels-of-detail. The number of vertices is reduced at each iteration, so that $\left|V_{i}\right|>\left|V_{i+1}\right|$. Ideally, each coarsened network should preserve the crucial (for the task) topological properties of its predecessor network.

It is worth noting that community detection and coarsening are not equivalent. A community detection algorithm does not require defining a number of reduction levels, a reduction rate or any other parameter to fit a desired network size or topology. Moreover, it returns a clustering of the, or equivalently a membership array. A coarsening algorithm, in contrast, produces a sequence of progressively simplified networks, which may be used to solve multiple tasks, community detection included.

The coarsening process involves a matching step, which defines which vertices will be merged and how, and a contraction step which builds the reduced representation, given the merged vertices. Choosing a suitable matching strategy is a key component of an effective multilevel optimization algorithm. An inadequate matching may degrade the quality of the 
initial solution obtained in the coarsest network, or the computational performance of the target algorithm, perhaps both. Given the complexity and importance of matching, many algorithms have been developed, which are reviewed next.

\subsubsection{Matching}

A matching $M=\left\{V_{k}\right\}_{k=0}^{K}$ is a partition of $V$ into a set of $K$ non-empty and disjoint subsets $V_{i} \in V$ such that $V=\bigcup V_{i}, 1 \leq\left|V_{i}\right|, \forall i$ and $i \neq j \Rightarrow V_{i} \cap V_{j}=\varnothing$. A set of restrictions defines how vertices can be matched, e.g. if vertices are to be paired the restriction is $\left|V_{i}\right|=2$, $\forall i$. A vertex $u \in V_{i}$ is called matched, otherwise, if $\nexists V_{i} \in M \mid u \in V_{i}, u$ is called unmatched, or a singleton, as illustrated in Fig. 5.

Parameter $\rho$, which defines the (maximum) reduction factor, controls the size of the matched set $|M|=\left|\cup V_{i}\right|=\sum\left|V_{i}\right|$. If vertices are matched pairwise, then $\sum\left|V_{i}\right|=\lceil\rho|V|\rceil$, i.e., the number of vertices to be matched is given by the reduction factor $(\rho \in(0,0.5])$ multiplied by the network size $|V|$. Considering, for instance, a network $G$ with $|V|=100$ and $\rho=0.3$, then $\sum\left|V_{i}\right|=60$ vertices will be selected, or 30 vertex pairs. If $\rho=0.5$, the number of vertices decreases by a factor of (at most) two at each iteration, yielding a logarithmic decrease in the size of the coarsened networks. Alternatively, higher reduction rates are possible adopting greater values of $\rho\left(\rho \in(0.5,1] \Rightarrow \exists i:\left|V_{i}\right|>2\right)$; or $\rho$ may be set so as to obtain a coarsest network with a desired number of vertices after executing $L$ iterations.

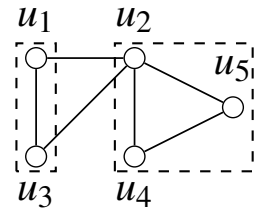

(a)

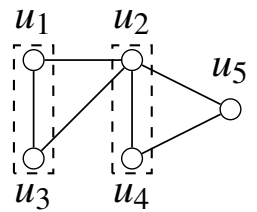

(b)

Figure 5 - Two examples of matching: (a) matching $M=\left\{\left(u_{1}, u_{2}\right),\left(u_{3}, u_{4}, u_{5}\right)\right\}$, with no singletons and groups of sizes 2 and 3; (b) pairwise matching $M=\left\{\left(u_{1}, u_{2}\right),\left(u_{3}, u_{4}\right)\right\}$ with $u_{5}$ as a singleton.

Source: Elaborated by the author.

We introduce, as one of the contributions of this paper, a systematic organization of the matching strategies according to their objective, restrictions, and limitations. They have been grouped into three categories, namely edge-selection, vertex-selection, and cluster-selection (or community-selection), summarized in Table 6, and are further detailed in the remainder of this section.

Early matching strategies did edge-selection, in which the aim is to find an independent edge set $E_{s}$ such that no two edges are adjacent. The underlying rationale is to collapse pairs of adjacent vertices (incident to the same edge $e_{i} \in E_{S}$ ), whereas maintaining isolated vertices unmatched, i.e. $E_{s} \Rightarrow V_{i}, \forall i$, therefore the matching $M$ is entailed by $E_{s}$. Fig. 6 illustrates an 
Table 6 - Taxonomy of the matching strategies.

\begin{tabular}{|c|c|c|c|}
\hline Category & Objective & Restriction & Limitations \\
\hline $\begin{array}{l}\text { Edge- } \\
\text { selection }\end{array}$ & $\begin{array}{l}\text { Find an indepen- } \\
\text { dent edge set }\end{array}$ & $\begin{array}{l}\text { Collapse pairs of adjacent } \\
\text { vertices }\end{array}$ & $\begin{array}{l}\circ \text { Isolated vertices. } \\
\circ \text { Scale-free networks. } \\
\circ \text { Network with a small number of } \\
\text { adjacent edges (or very sparse } \\
\text { networks). } \\
\circ \text { K-partite and heterogeneous net- } \\
\text { works. } \\
\circ \text { Can break some topological } \\
\text { structures such as communities } \\
\text { and cliques. }\end{array}$ \\
\hline $\begin{array}{l}\text { Vertex- } \\
\text { selection }\end{array}$ & $\begin{array}{l}\text { Find independent } \\
\text { pairs of vertices }\end{array}$ & $\begin{array}{l}\text { Collapse pairs of adjacent } \\
\text { and non-adjacent vertices }\end{array}$ & $\begin{array}{l}\triangleright \text { The size of a local neighborhood } \\
\text { is a complex parameter. } \\
\triangleright \text { Can degrade topological struc- } \\
\text { tures such as communities and } \\
\text { odd cliques. }\end{array}$ \\
\hline $\begin{array}{l}\text { Cluster- } \\
\text { selection }\end{array}$ & $\begin{array}{l}\text { Find groups of } \\
\text { vertices }\end{array}$ & Collapse groups of vertices & $\begin{array}{l}\diamond \text { Few coarsening levels or lack of } \\
\text { hierarchical structure. } \\
\diamond \text { Slower than edge and vertex- } \\
\text { selection. }\end{array}$ \\
\hline
\end{tabular}

edge-selection matching, in which edges $\left(u_{1}, u_{2}\right)$ and $\left(u_{4}, u_{5}\right)$ have been selected, causing the pairs $\left\{u_{1}, u_{2}\right\}$ and $\left\{u_{4}, u_{5}\right\}$ to be merged and vertex $u_{3}$ to remain unmatched.

Finding an optimal maximal $E_{s}$ is a computationally expensive problem, typically approached combining a meta-heuristics - which can be relatively fast and accurate with linear or near-linear time complexity - with diverse search strategies against the solution space. Edgeselection may, for instance, consider only adjacent vertices for matching, leaving isolated vertices unmatched. This yields poor results on sparse networks, as the number of isolated super-vertices increases quickly as the coarsening proceeds, possibly converging to a zero edge network in the final stages. Such strategies can only afford aggressive reduction rates in networks with many non-adjacent edges, which is hardly the case of highly sparse networks.

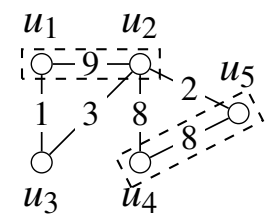

Figure 6 - Edge-selection matching (ESM) strategy that yields a matching $M=\left\{\left(u_{1}, u_{2}\right),\left(u_{4}, u_{5}\right)\right\}$.

Source: Elaborated by the author.

Similarly, the number of adjacent edges available for matching decreases dramatically when coarsening scale-free networks, which have few hubs and many low-degree vertices. Once a hub is matched, its low-degree neighbors are unlikely to find a match later. This limitation is illustrated in Fig. 7, where an edge-selection strategy would match the pair $\left\{u_{1}, u_{8}\right\}$, causing the remaining vertices connected to $u_{8}$ to remain unmatched until the subsequent coarsening 
level. In these scenarios, reduction rates can be very slow (ABOU-RJEILI; KARYPIS, 2006), canceling out the potential benefits of a multilevel strategy.

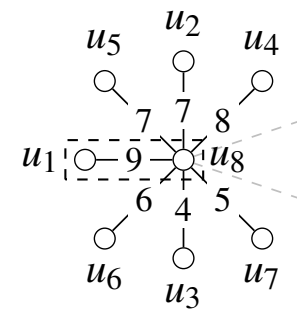

Figure 7 - Edge-selection strategy that selects a matching $M=\left\{\left(u_{1}, u_{8}\right)\right\}$.

Source: Elaborated by the author.

Standard edge-selection methods are not directly applicable to bipartite, or to general k-partite networks, in which edges can only connect vertices in distinct layers. If adjacent vertices were matched, as illustrated in Fig. 8, they would create super-vertices connected to vertices in multiple layers, transforming the bipartite or k-partite network into an heterogeneous network.

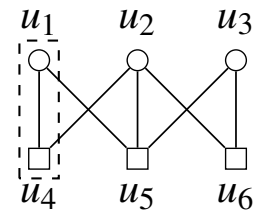

(a)

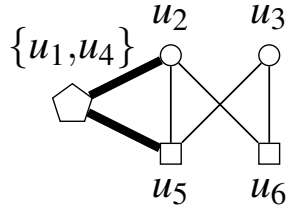

(b)

Figure 8 - Prohibitive matching case. In (a), the pair of adjacent vertices $\left\{u_{1}, u_{4}\right\}$ is selected as a match, causing the original bipartite network to be coarsened into the heterogeneous network in (b).

Source: Elaborated by the author.

Indeed, in certain situations it may be more convenient to match a pair of non-adjacent vertices than a pair of adjacent ones. For instance, two vertices with many neighbors in common are more likely to belong to a highly-connected sub-network, being thus interesting candidates for matching. Fig. 9 illustrates an example in which the matching is defined based on the number of common neighbors between two vertices, $S_{c n}(u, v)$. Let $u_{1}$ be a randomly selected vertex, then, $S_{c n}\left(u_{1}, u_{2}\right)=0, S_{c n}\left(u_{1}, u_{3}\right)=0, S_{c n}\left(u_{1}, u_{4}\right)=1, S_{c n}\left(u_{1}, u_{5}\right)=1, S_{c n}\left(u_{1}, u_{6}\right)=4, S_{c n}\left(u_{1}, u_{7}\right)=1$ and $S_{c n}\left(u_{1}, u_{8}\right)=2$. Then, $u_{1}$ and $u_{6}$ would be the best choice for a match. An edge-selection strategy would select the pair $\left\{u_{1}, u_{8}\right\}$, since it selects the best $S_{c n}$ from the neighbors of $u_{1}$.

Vertex-selection matching has been introduced to overcome limitations of edge-selection strategies. Again, the goal is to find a set of paired vertices, but in vertex-selection both adjacent or non-adjacent vertices can be matched; thus even isolated vertices can find a match. Fig. 10 illustrates a vertex-selection matching strategy, let us consider $S_{c n}(u, v)$ as the matching criterion and $u_{1}$ the vertex randomly selected for matching. The pair of non-adjacent vertices $\left\{u_{1}, u_{6}\right\}$ is matched, since $S_{c n}\left(u_{1}, u_{6}\right)=4$ is the best choice. However, the cost of matching non-adjacent 
vertices in large-scale networks is prohibitive, since all possible vertex pairs must be inspected. A frequent strategy is to reduce the search space to a local neighborhood, as illustrated in Fig. 11. It may be reasonable, for instance, to explore the local 2-hop neighborhood of each vertex (VALEJO et al., 2018b).

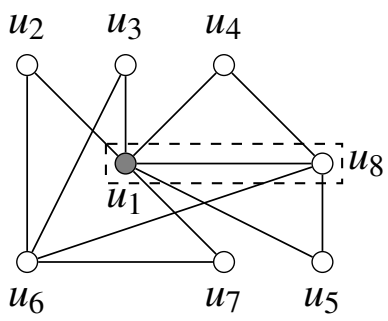

Figure 9 - Choices for matching a randomly selected vertex $u_{1}$ : it would be matched with $u_{6}$ if the matching criterion considered the vertex with more common neighbors; it is matched with the adjacent vertex $u_{8}$ if adopting an edge-selection strategy.

Source: Elaborated by the author.

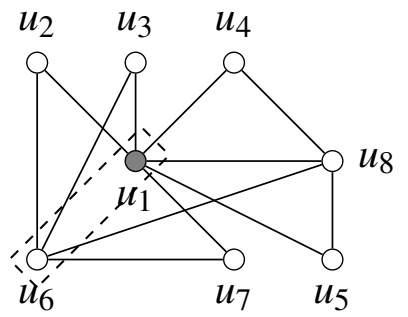

Figure 10 - Vertex-selection matching strategy in which the criterion is the number of common neighbors. The randomly selected vertex $u_{1}$ will be matched with non-adjacent vertex $u_{6}$, as they have more neighbors in common within all possible pairs of $u_{1}$.

Source: Elaborated by the author.

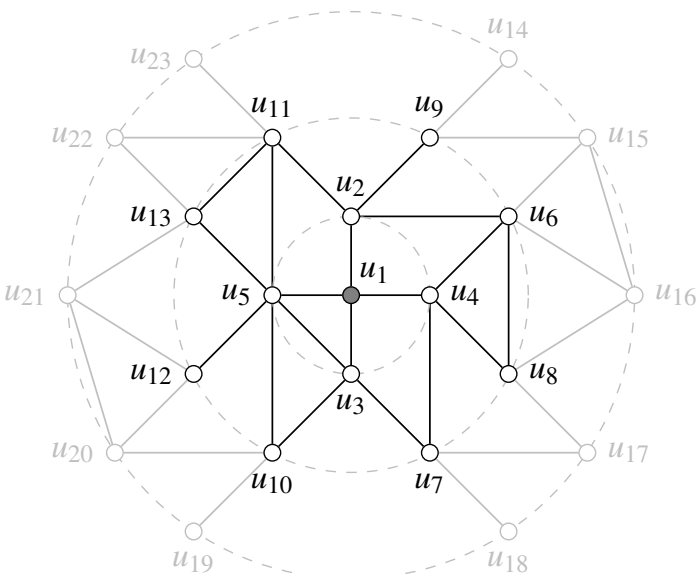

Figure 11-Local 2-hop neighborhood selection. The vertex $u_{1}$ may be matched with its neighbors and neighbors of neighbors, i.e. with any vertex in the set $V^{\prime}=$ $\left\{u_{2}, u_{3}, u_{4}, u_{5}, u_{6}, u_{7}, u_{8}, u_{9}, u_{10}, u_{11}, u_{12}, u_{13}\right\}$.

Source: Elaborated by the author. 
Although considering local neighborhoods reduces the cost of edge and vertex-selection strategies, pairwise matching of vertices may introduce major drawbacks. At each coarsening level, the vertices selected first are more likely to find a high-quality match than those selected later, which may introduce and propagate inconsistencies into the further coarsening levels. Let us consider, as an illustration, networks with community structures - where the communities consist of cohesive groups of "densely" connected vertices which are sparsely connected to vertices outside the group. If vertices from distinct communities can be matched, the original community structures will gradually degrade. Fig. 12a illustrates the problem, in which the pair $\left\{u_{5}, u_{6}\right\}$ has been matched with an edge-selection strategy. Although it causes no harm at the current level, this matching will likely yield a poor quality coarser network at a later stage. Edge and vertex selection criteria can also degrade $k$-cliques with odd values of $k$. In the network illustrated in Fig. $12 \mathrm{~b}$ a vertex selection strategy added the pair $\left\{u_{3}, u_{4}\right\}$ to the matching. This choice will degrade the clique structures at the next level, since now a single super-vertex includes vertices from distinct 3-cliques. In both cases the "quality" criterion is known a priori, so that a "high-quality matching" may be defined as one that prioritizes matching vertices in the same community or $k$-clique, whereas an "inconsistent matching" is one that ignores the corresponding quality criteria.

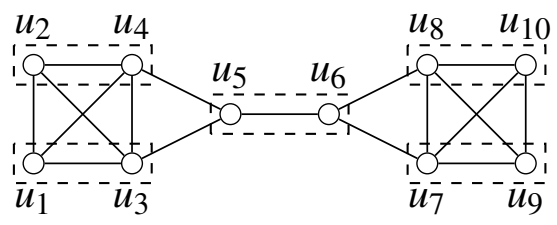

(a)

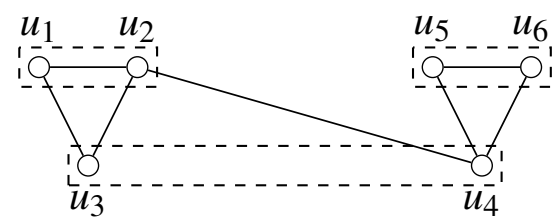

(b)

Figure 12 - Edge and vertex-selection matching strategies on networks with topological structures such as communities and cliques. In (a) the pair of vertices $\left\{u_{5}, u_{6}\right\}$ are matched using an edgeselection strategy. In (b) a vertex-selection strategy matches the pair $\left\{u_{3}, u_{4}\right\}$. These choices may degrade the topology of the coarser networks.

Source: Elaborated by the author.

Furthermore, if vertices can only be matched pairwise, the maximum network reduction rate possible at each coarsening level is $50 \%$. Expressive reduction of large networks would require many coarsening levels with high processing and memory costs. In these scenarios cluster-selection matching strategies, which match groups of vertices with common properties or with similar roles, adjacent or not, are more adequate. Matched groups, i.e., super-vertices, may have arbitrary size or their size may be constrained in order to enforce balanced groups. This flexibility allows higher network reduction rates, e.g. on the order of $60 \%-90 \%$ at each level, depending on the size constraints imposed. Furthermore, even vertices already matched may be considered in assessing a potential best match, avoiding inadequate choices that may degrade existing topological structures. Fig. 13 illustrates a cluster-selection matching strategy and its potential advantages in two distinct networks. 


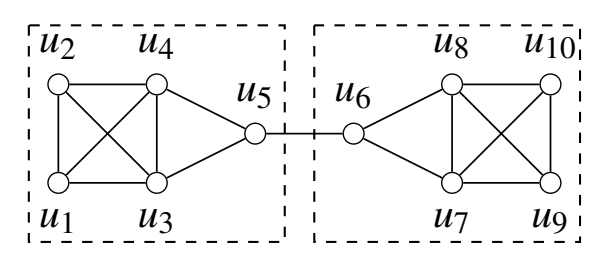

(a)

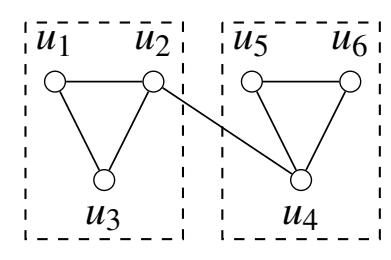

(b)

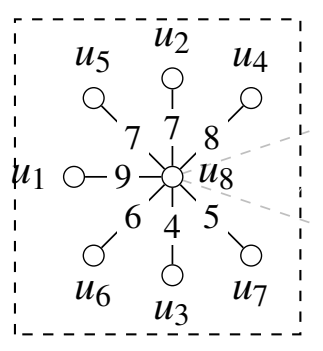

(c)

Figure 13 - Cluster-selection matching strategy applied to a network with community (a) and clique (b) structures and to a scale-free network (c). The matching choices define clusters which preserve the original structures into the super-vertices.

Source: Elaborated by the author.

Despite its potential advantages, cluster-based matching requires some caution. Very high network reduction factors imply in few coarsening levels (maybe even a single one), with negative impact on the effectiveness of the refinement phase. In order to enforce a gradual reduction, essential to the success of the multilevel method, one may impose an upper threshold on the reduction factor or limit super-vertex sizes. Moreover, the computational cost of clusterbased matching is, in general, higher than that of edge or vertex-selection strategies, which may render it unfeasible in real-time applications. The choice of matching strategy is highly problem dependent. In the following subsections we review the matching algorithms most representative of each strategy.

\subsubsection{Edge-selection matching algorithms (ESM)}

Karypis and Kumar (1995b), Karypis and Kumar (1998a), Karypis and Kumar (1998b) introduced the first algorithms for edge-selection matching (ESM), illustrated in Fig. 14. In Random Matching (RM) the vertices are visited in random order. If a vertex $u$ is still unmatched, one of its unmatched neighbors $v$, if any, is selected arbitrarily and the pair $(u, v)$ is included in $E_{s}$, see Fig. 14a, otherwise $u$ remains unmatched. RM, albeit a fast and straightforward baseline strategy, may yield poor results in certain situations. The Heavy Edge Matching (HEM) is similar, but it imposes a precedence based on edge weights in order to minimize the total strength $S=\sum s_{i}$ of the coarsened network. In HEM a random vertex $v$ is matched with $u$ if edge $(v, u)$ has maximum weight over all edges incident to $v$, as illustrated in Fig. 14b. Compared with RM, HEM yields better results, with equivalent asymptotic complexity (KARYPIS; KUMAR, 1995b), although a minimum $S$ is not guaranteed (nor it is necessarily desired).

Unlike HEM, Light-Edge Matching (LEM) aims at maximizing $S$, matching a randomly selected vertex $v$ with its unmatched neighbor $u$ with minimum edge weight, as illustrated in $14 \mathrm{c}$. MHEM is a variation that minimizes the average degree of the coarsened network. A data structure $H_{u}$ keeps the set of unmatched vertices adjacent to $u$ with maximum edge weight (in case of identical weights $H_{u}$ can hold multiple vertices). Let $W_{v, u}$ be the sum of the edge weights 
between $v$ and common neighbors with $u$, i.e. $W_{v, u}=\sum_{x} w_{v, x} \mid e_{x, u}=1$, where $w$ is the edge weight. In MHEM, $v$ is matched with $u \in H$ with maximum $W_{v, u}$, as illustrated in Fig. 14d.

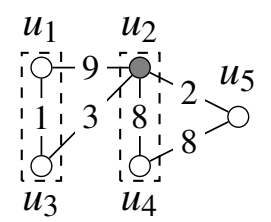

(a)

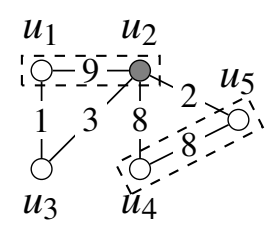

(b)

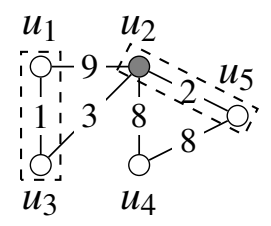

(c)

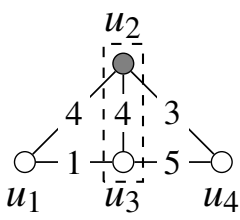

(d)

Figure 14 - Different edge-selection matching (ESM) strategies. Let $u_{2}$ be the randomly selected vertex to match (the start vertex): (a) RM selects a matching $\left.M=\left\{\left(u_{1}, u_{3}\right),\left(u_{2}, u_{4}\right)\right)\right\}$; (b) HEM yields a matching $M=\left\{\left(u_{1}, u_{2}\right),\left(u_{4}, u_{5}\right)\right\}$; (c) LEM yields a matching $M=\left\{\left(u_{1}, u_{3}\right),\left(u_{2}, u_{5}\right)\right\}$; and (d) MHEM yields a matching $M=\left\{\left(u_{2}, u_{3}\right)\right\}$.

Source: Elaborated by the author.

Karypis and Kumar (1998a) also introduced the Heavy-Clique Matching (HCM) algorithm. Again, vertices are visited in random order, and a pair $u$ and $v$ is matched if the sub-network induced by their common neighbors has a high clustering coefficient. Furthermore, Karypis and Kumar (1995b) introduced variations of RM, HEM and MHEM, respectively, called Sorted Random Matching (SRM), Sorted Heavy-Edge (SHE) and Sorted Modified Heavy-Edge (SMHE). These algorithms visit vertices in ascending order of their degree to reduce occurrence of unmatched vertices, since low-degree vertices are paired first.

These algorithms were later modified by Zhu et al. (2012) to incorporate matching criteria based on vertex similarity measures, such as the Number of Common Neighbors (NCN), the Average Number of Common Neighbors (ANCN), or the Weighted Average Number of Common Neighbors (WANCN). These variations gained wide acceptance. For a randomly selected vertex $u$, an adjacent pair $(u, v)$ is matched if $u$ and $v$ have a maximal number of common neighbors. The cost of computing and sorting the number of common neighbors for all pairs of adjacent vertices is $\mathscr{O}(m)$ and $\mathscr{O}(m \log m)$, respectively.

Computing an approximate solution to the problem of finding the maximum weighted independent edge set is feasile in near linear-time. For instance, the Greedy Heavy Edge Matching (GHEM), is a 1/2-approximation that achieves sub-optimal solutions, in which the total edgeweight is at least half the optimal, with $\operatorname{cost} \mathscr{O}(m \log n)$.

A drawback of the previous algorithms is that low-degree vertices tend to remain unmatched. The Gain Vertex Matching (GVM) is a variation of HEM that reduces the number of unmatched vertices along the sequence of coarsened networks (BOUHMALA; CAI, 2001); Fig. 15 illustrates the distinct approaches of GVM and HEM. In GVM a randomly selected vertex $u$ is matched with its unmatched neighbor $v$ that minimizes the strength of the supervertex yielded by $u$ and $v$. Thus, priority is given to low-degree vertices, e.g. in a network with equal-weight edges a vertex $u$ will be matched with its neighbor of lowest strength. 


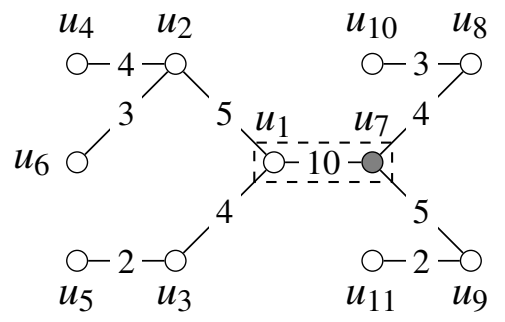

(a)

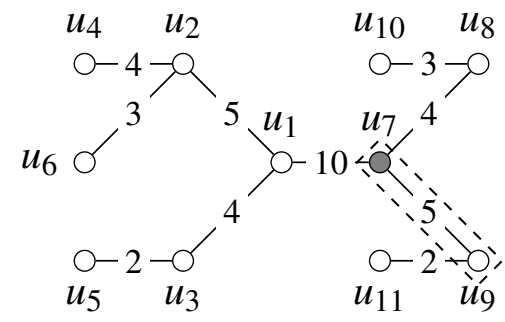

(b)

Figure 15 - Difference between the GVM and HEM matching algorithms. Let $u_{7}$ be the vertex to match: (a) HEM selects the pair with greater weight $\left(u_{1}, u_{7}\right)$; (b) GVM selects the pair that yields the minimum-strength super-vertex, $\left(u_{1}, u_{9}\right)$.

Source: Elaborated by the author.

Other algorithms rely on path growing heuristics to compute a matching with maximum weigh. The path growing algorithm (PGA), introduced by Drake and Hougardy (2003b) and later improved by Drake and Hougardy (2003a), is a 1/2-approximation strategy achieved by means of growing disjoint vertex paths with cost $\mathscr{O}(n)$. Alternatively, the Global Path Algorithm (GPA) (MAUE; SANDERS, 2007) is a synthesis of GHEM that scans the edges in descending order of weight and applies the aforementioned path heuristic with an applicable edge restriction. An edge is applicable if it connects two vertices common to different paths or common vertices of an odd-length path; it is not applicable if it closes an odd length cycle or is incident to a vertex of an existing path.

Following a similar rationale, Monien, Preis and Diekmann (2000) presented a linear 1/2-Approximation Matching (LAM) method to find the maximum weighted independent edge set, with time complexity $\mathscr{O}(n)$. The algorithm is similar to GHEM, but adopts a local strategy. It starts with a randomly selected edge and iterates over the remaining adjacent edges. If a heavy adjacent edge can be found, the algorithm switches to the new edge and repeats the process until reaching a locally heaviest edge. Therefore, edge weights increase along the path.

Other methods start with some selected vertices, but rather than adopting parameter $\rho$ as a stopping criterion they set a desired number of central vertices that will become super vertices. Strategies based on central vertices (also called "seeds" or "centroids") rely on the principle that some vertices are "leaders" and the remaining are "followers", therefore, super-vertices are built around the leaders. Central vertices are often selected based on some centrality measure, such as preferential attachment. Erciye, Alp and Marshall (2005) proposed a Fixed Centered Matching (FCM). Initially, the central vertices must be selected. If time is not an issue, it is possible to run a standard Breadth-First-Search (BFS) algorithm, which is $\mathscr{O}(|V|+|E|)$ with $\mathscr{O}(|E|)$ between $\mathscr{O}(1)$ and $\mathscr{O}\left(\left|V^{2}\right|\right)$, to find $p$ central vertices at a maximum distance from each other. Otherwise, the central vertices can be chosen randomly, with no constraints or constrained to being at a predetermined distance from each other. Once they are chosen, for each center $u$ an adjacent vertex $v$ is selected, e.g. employing HEM or RM. The method is linear time if central vertices are 
selected randomly. Authors state that FCM is directly applicable as a partitioning algorithm, i.e., considering each super-vertex as a partition.

Although the aforementioned matching methods consider edge-weights, experimental results suggest more sophisticated edge-rating functions may provide better criteria for producing more accurate and robust coarsened representations (SAFRO; RON; BRANDT, 2006; HOLTGREWE; SANDERS; SCHULZ, 2010; CHEN; SAFRO, 2011; SANDERS; SCHULZ, 2011; SAFRO; SANDERS; SCHULZ, 2012; BRANNICK et al., 2013; GLANTZ; MEYERHENKE; SCHULZ, 2014; PUROHIT et al., 2014; DIAS et al., 2017). Such strategies adopt a function that assigns a rating to each network edge, which may replace the edge weights, or may be handled as an additional edge attribute. Then, a matching strategy attempts to maximize the sum of the ratings of the edges to be matched. Edge rating functions can be integrated into classical matching algorithms, such as GPA, HEM and FCM.

\subsubsection{Vertex-selection matching algorithms (VSM)}

The most popular vertex-selection matching strategy was first introduced and later improved by Walshaw (WALSHAW, 2001b; WALSHAW, 2004; WALSHAW, 2008b). The original algorithm visits vertices in descending order of their degree and each visited vertex $u$ is matched with an unmatched neighbor $v \in \Gamma_{2}(u)$ for which the number of common neighbors is maximum, as illustrated in Fig. 16a.

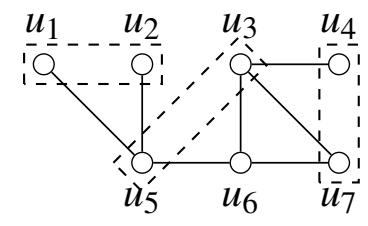

(a)

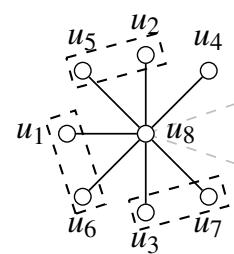

(b)

Figure 16 - Variations of the VSM matching: a and b depict the classic and the star-like approaches, respectively.

An important use of the vertex-selection strategy is to select vertices in star-like structures, in which a high-degree central vertex, called hub, is connected to many peripheral low-degree vertices. This structure is found in scale-free networks with a power-law degree distribution. The rationale is to match pairwise the vertices adjacent to a single hub, as discussed in Chen et al. (2018) and illustrated in Fig. 16b.

Valejo et al. (2017) and Valejo et al. (2018a) proposed a vertex-selection matching for bipartite networks based on their one-mode projections (OPM). A bipartite network $G=$ $\left\{V_{1} \cup V_{2}, E\right\}$ can be transformed into two unipartite networks, $G_{1}$ and $G_{2}$, relative to each layer, in which vertices with common neighbors in the bipartite network are connected by edges in their respective projection. The edge-selection matching is executed in the one-mode projection and the 
matching thus obtained is projected back to the original bipartite network, as illustrated in Fig. 17. After computing the one-mode projections of each layer, $G_{1}$ and $G_{2}$, the RM edge-selection is applied to both, which yields the matching $M=\left\{\left\{u_{1}, u_{2}\right\},\left\{u_{3}, u_{4}\right\},\left\{u_{5}, u_{6}\right\}\right\}$. Finally, $M$ is projected back to the original bipartite network. Although it performs edge selection the method is categorized as a vertex-selection matching, as the final matching consists of non-adjacent vertices.

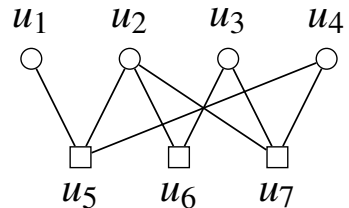

(a) Original bipartite network

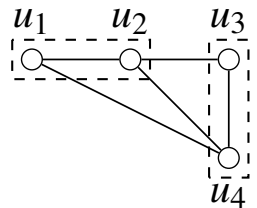

(b) One-mode projection $G_{1}$ and $G_{2}$

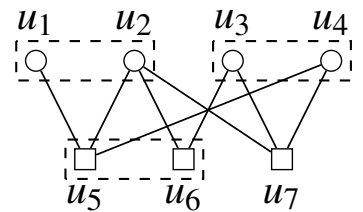

(c) Projected matching

Figure 17 - Vertex-selection matching for bipartite networks based on its one-mode projections.

Source: Elaborated by the author.

Also introduced for bipartite networks, the GMb and RGMb matching algorithms adopt a local 2-hop neighborhood restriction, implying that adjacent vertices are not matched (VALEJO et al., 2018b). Furthermore, vertices can only match others in their 2-hop neighborhood set, rather than any non-adjacent vertex, which reduces the search space and the computational cost. Considering, for instance, the network in Fig. 18a, in a first iteration a selected vertex $u_{1}$ can match vertices $u_{2}$ and $u_{4}$, which are non-adjacent and are in its 2-hop neighborhood. In this case the pair $\left\{u_{1}, u_{2}\right\}$ is matched. In the following iteration, illustrated in Fig. 18b, a selected vertex $u_{2}$ can match vertices $u_{2}$ and $u_{4}$. Since $u_{2}$ has already been matched, $u_{4}$ is the remaining choice of matching for $u_{3}$.

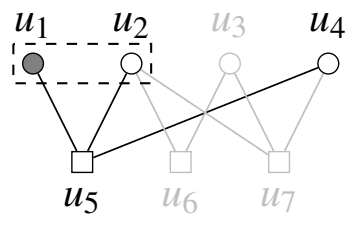

(a)

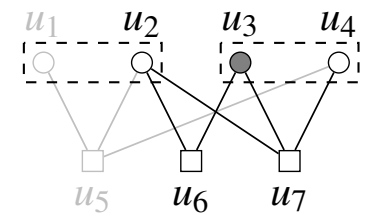

(b)

Figure 18 - Bipartite network, such that $V_{1}=\left\{u_{1}, u_{2}, u_{3}, u_{4}\right\}$ and $V_{2}=\left\{u_{5}, u_{6}, u_{7}\right\}$; (a) in a first iteration of a vertex-selection strategy the pair $u_{1}, u_{2}$ is included in the matching; (a) in the next iteration the pairs $\left\{u_{3}, u_{4}\right\}$ is included in the matching.

Source: Elaborated by the author.

\subsubsection{Cluster-selection matching algorithms (CSM)}

Abou-Rjeili and Karypis (2006) introduced the first cluster-selection matching, an approach that matches vertex sets of arbitrary size while ensuring the network will be reduced by a 
given ratio. An unmatched vertex $u$ can be matched with any of its neighbors $v$, including those already in the matched set, causing groups of vertices to be collapsed together, revisit Fig. 13. In contrast, Chernoskutov, Ineichen and Bekas (2015) considered betweenness centrality to select the $k$-top vertices as fixed centers, which they call hubs. Each fixed center is matched with all its neighbors independently of edge-weight. Small values of $k$ imply in a small network reduction ratio, whereas high values can build sparse networks and yield faster network reduction in the subsequent levels.

The paper introducing the Louvain multilevel community detection algorithm has inspired several cluster-selection matching strategies Blondel et al. (2008). It relies on modularity optimization and resembles the well-known FastGreedy (CLAUSET; NEWMAN; MOORE, 2004) algorithm for modularity maximization. Louvain assigns each vertex to a distinct community and communities can be interpreted as super-vertices. It returns a membership array and is not a matching algorithm strictly speaking, although deriving a matching algorithm directly applicable to network coarsening is straightforward.

Modularity maximization, possibly with slightly modified formulations of the modularity score, can also be employed for cluster-based matching, as introduced by Arenas et al. (2007) in an important theoretical contribution. Efforts are, in general, directed towards devising alternative strategies to iterate on the network structure and towards establishing criteria to control cluster growth. LaSalle and Karypis (2015) propose reducing the search space to a local neighborhood considering only adjacent vertices. The Multi-Step Greedy matching method introduced by Schuetz and Caflisch (2008a), Schuetz and Caflisch (2008b) and later improved by Noack and Rotta (2009) avoids extremely unbalanced clusters. The matching introduced by Huang et al. (2010) combines the advantages of density-based clustering and modularity-based optimization methods.

A near-linear time matching $-\mathscr{O}(n+m)$ in each iteration - based on the LP (label propagation) community detection algorithm (RAGHAVAN; ALBERT; KUMARA, 2007) has been introduced by Meyerhenke, Sanders and Schulz (2014). Overall, the strategy creates supervertices by propagating labels throughout the network and collapses vertex groups, rather than pairs. Initially, every vertex is assigned a unique label (an identifier). At each iteration, each vertex updates its label with the dominant one, i.e. the most frequent in its neighborhood. Intuitively, densely connected groups of vertices will converge to a single dominant label. Finally, vertices with the same assigned label at convergence will be collapsed into a super-vertex, Fig. 19a. Wang et al. (2014a) describe an LP-based matching implementation in a distributed memory system capable of partitioning a network with billion vertices within a few hours.

$k$-cliques are ubiquitous topological structures in complex networks and sometimes considered in defining super-vertices. The cost of finding $k$-cliques rises exponentially with network size, but 3 -cliques (also called triangles) can be found with cost $\mathscr{O}\left(|E|^{2} /|V|\right)$. The strategy is illustrated in Fig. 19b. Feder and Motwani (1995) developed a matching based on 
biclique contraction in bipartite networks. Gupta (1997), and later Oliveira and Seok (2007a), Kang et al. (2015b) and Kang et al. (2015a), proposed methods to contract each triangle into a super-vertex, differing essentially in the clique-search strategy and the data structures employed. Gupta (1997) presents a fast and simple heuristic in which a randomly selected vertex is matched with the two neighbors that yield the maximum sum of edge-weights.

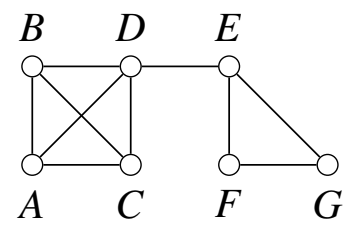

(a)

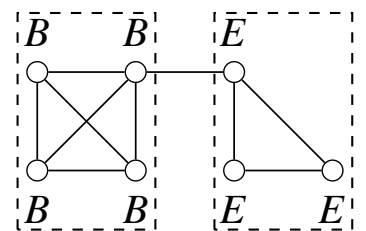

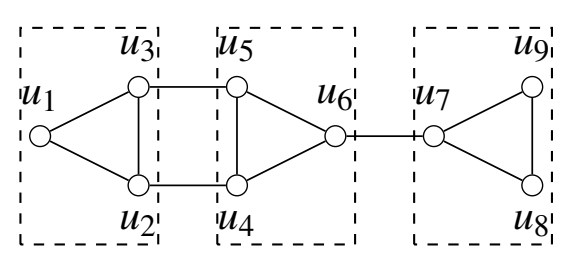

(b)

Figure 19 - Cluster-selection matching based on label propagation (a) and triangle definition (b), respectively.

Some solutions adopt an upper bound to ensure that the super-vertices satisfy a sizeconstraint. In the standard strategies, at each coarsening level heavier super-vertices tend to collapse with their lighter neighboring vertices and super-vertices, so they become even heavier and are given higher matching priority in subsequent coarsening levels. Fig. 20 illustrates the problem on a hypothetical scenario, in which the vertex pair $\left\{u_{1}, u_{2}\right\}$ is given priority over the clique $\left\{u_{2}, u_{3}, u_{4}, u_{5}\right\}$, even though both have the same number of common neighbors. It may be useful, in some applications, to constrain super-vertex size or ensure they are balanced regarding some property. Yao et al. (2011) improved the HEM algorithm by re-collapsing matched vertices with unmatched ones, which allows collapsing groups of vertices together. In order to enforce super-vertex balance the re-collapsing process will exclude any matched vertex whose weight exceeds $|V| / k$, wherein $k$ is the number of vertex groups. Meyerhenke, Sanders and Schulz (2014) extended the LP matching algorithm to ensure that a super-vertex must not exceed $(1+\varepsilon)\left\lceil\frac{|V|}{k}\right\rceil$ wherein $k$ is the number of vertex groups and $\varepsilon$ is a tuning parameter. Akhremtsev, Sanders and Schulz (2015) also improved the LP matching algorithm and used the memory size to constrain super-vertex size, i.e., a super-vertex must fit into the internal memory and is split into smaller groups if this restriction is not met.

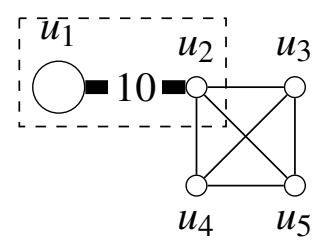

Figure 20 - The priority choice $\left\{u_{1}, u_{2}\right\}$ over the alternative $\left\{u_{1}, u_{2}, u_{3}, u_{4}\right\}$ will produce a highly unbalanced super-vertex. 


\subsubsection{Contraction}

The coarsening phase comprises matching and contraction, a convenient division that renders both tasks independent and more amenable to adaptations. From the discussion so far, it is evident that there is a vast literature on the matching task. As opposite, a general algorithm suffices to describe the contraction task, responsible for constructing the reduced network from a given matching.

Given a matching, the contraction algorithm collapses each group of matched vertices $\left\{u_{1}, \ldots, u_{n}\right\}$ into a single super-vertex $s V_{i}$. A successor-network $G_{l+1}$ will inherit the nonmatched vertices from its predecessor-network $G_{l}$. For $G_{l+1}$ to be a good approximation to its predecessor-network, given a super-vertex $s V_{i}=\left\{u_{1}, \ldots, u_{n}\right\} \in V_{l+1}$ its weight $\sigma\left(s V_{i}\right)$ is given by the sum of the weights of its predecessor-vertices. Furthermore, any edges incident to vertices $\left\{u_{1}, \ldots, u_{n}\right\} \in V_{l}$ are collapsed into the so-called super-edges incident to $s V_{i}$. Supervertices and super-edges are called successor-vertices and successor-edges of its originating vertices and edges, respectively. Vertices $\left\{u_{1}, \ldots, u_{n}\right\} \in V_{l}$ and edges $\left\{e_{1}, \ldots, e_{n}\right\} \in E_{l}$ collapsed into a super-vertex $s V_{i} \in V_{l+1}$ and a super-edge $s E_{i} \in E_{l+1}$ are called predecessor-vertices and predecessor-edges of $s V_{i}$ and $s E_{i}$, respectively.

Algorithm 2 describes the procedure to build a coarser network from an input network $G_{l}$ and a matching $M$ stored in a simple array.

Initially, the vertex pairs, or groups, are mapped into a successor vector (lines 4-10) and collapsed into a single super-vertex $s V \in G_{l+1}$ (line 7). The successor vector will be accessed again in the uncoarsening phase to project the solution relative to its super-vertex $s V$. Any vertices not included in $M$ are inherited by $G_{l+1}$ (line 12). After the mapping, adjacent edges in $E_{l}$ are collapsed into a single edge and added to $E_{l+1}$ (lines 13-19), i.e. each pair $(u, v) \in E_{l}$ entails a sucessor $(w, z)$. If the successor is already in $E_{l+1}$, its weight is increased by $\omega_{l}(u, v)$ (lines 16-17); otherwise, a new edge $(w, z)$ with weight $\omega_{l}(u, v)$ is inserted into $E_{l+1}$ (lines 18-19).

Coarser networks are, by definition, weighted (if the initial network is unweighted the super-edge weights will hold the edge count), but super-edge weights may be ignored if not relevant to the problem. Algorithm 2 computes super-edge weights directly as the sum of the predecessor-edges. Alternative approaches to compute super-edge weights have been little explored so far and could be further investigated. It may be interesting, for instance, to apply normalization factors, e.g., consider the number of edges in the predecessor-network, weakening the importance of edges connecting vertices with popular matches, such as hubs.

Fig. 21 illustrates a network $G_{l}$ and its coarser version $G_{l+1}$ obtained with the matching $M=\left\{\left\{u_{1}, u_{3}\right\},\left\{u_{2}, u_{4}\right\},\left\{u_{8}, u_{10}\right\},\left\{u_{7}, u_{9}\right\}\right\}$, in which all matched vertex pairs have been collapsed into a corresponding super-vertex. Fig. 22a illustrates a coarser network $G_{l+1}$ obtained from the network $G_{l}$ and the cluster-selection matching given by $M=\left\{\left\{u_{1}, u_{2}, u_{3}, u_{4}, u_{5}\right\}\right.$, $\left.\left\{u_{6}, u_{7}, u_{8}, u_{9}, u_{10}\right\}\right\}$. 


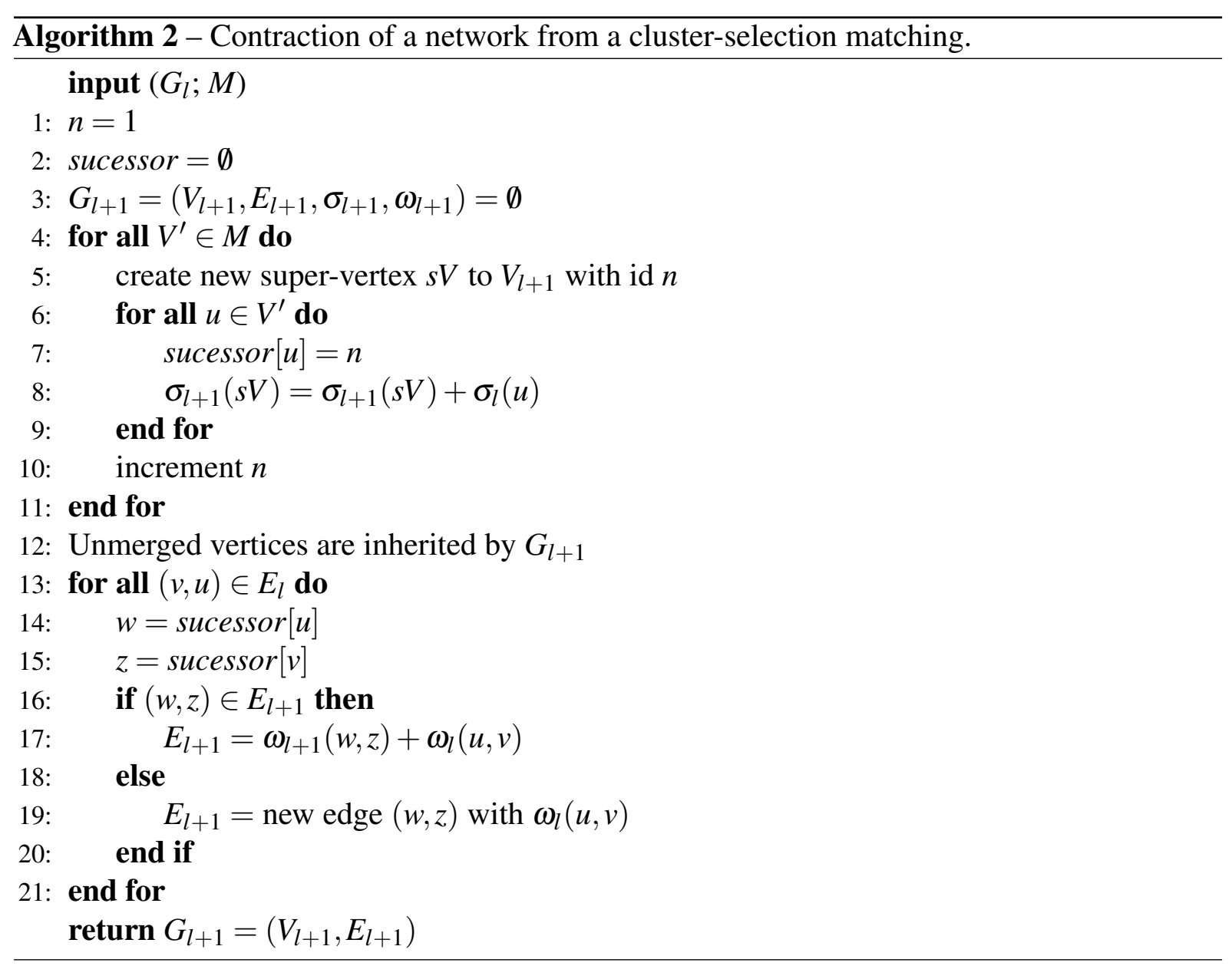

The contraction process may or not allow the presence of self-loops (i.e., edges that connect a vertex to itself), although in general they are suppressed. Although this definition is still allusive, some authors consider a graph with self-loops as a multigraph. Otherwise, the self-loop is considered as a special network edge. Fig. 21b and Fig. 22a show a coarsened network with four and with two self-loops, respectively. To suppress the self-loop in Algorithm 2, a super-edge (lines 16-19) must be created only if $w \neq z$.

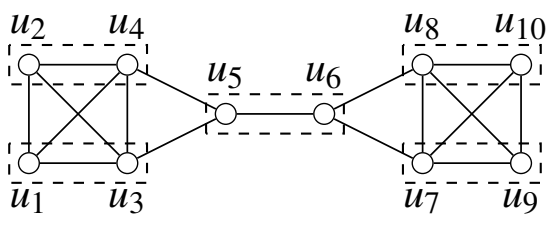

(a) $G_{l}$

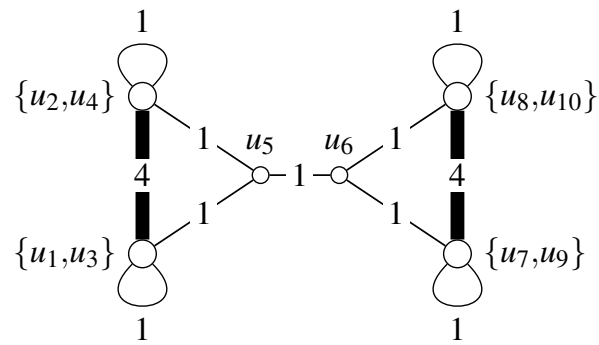

(b) $G_{l+1}$

Figure 21 - Result from a coarsening process based on an edge-selection or a vertex-selection matching strategies.

The standard contraction algorithm can be easily adapted to handle other network types, e.g., Karger and Stein (1993) modified it to handle multigraphs. In this case, super-vertices 
are created as usual (lines 1-12), i.e., matched vertices are collapsed into super-vertices and unmatched vertices are inherited by the successor-network. In contrast, rather than creating the super-edges (lines 13-19), all edges incident to predecessor-vertices are inherited by the successor-network, preserving the parallel edges and the multigraph structure. Fig. 22b depicts a multigraph $G_{l}$ and its coarser version $G_{l+1}$ obtained with the matching $M=\left\{\left\{u_{1}, u_{2}\right\}\right\}$, in which the matched vertices have been collapsed into a super-vertex and all edges in $G_{l}$ are inherited by $G_{l+1}$.

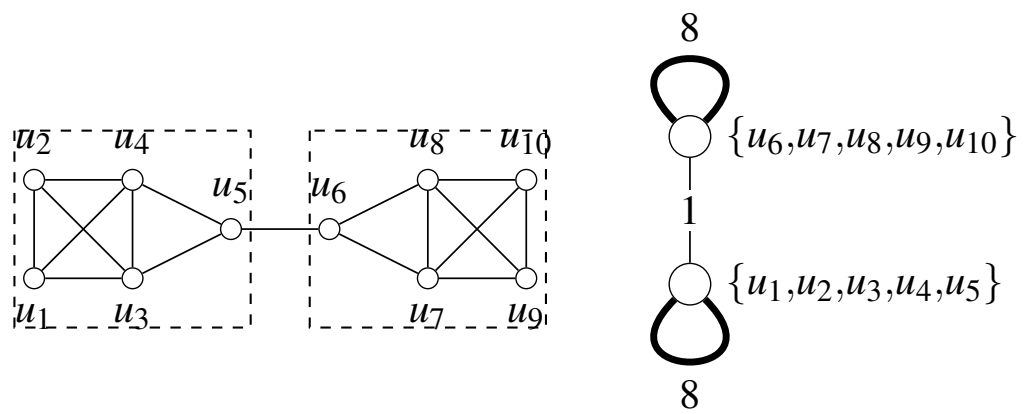

(a) $G_{l}$ and $G_{l+1}$
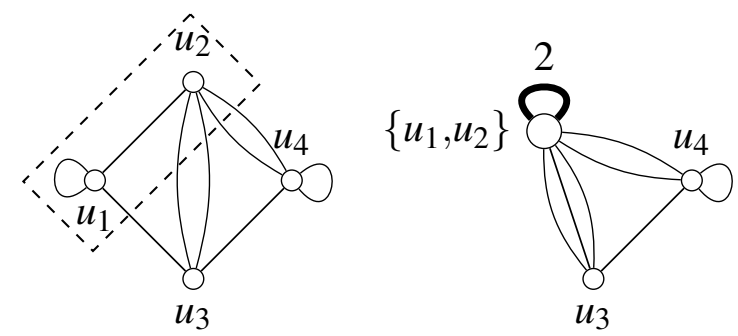

(b) $G_{l}$ and $G_{l+1}$

Figure 22 - In (a): result from a contraction process based on a cluster-selection matching strategy. In (b): result from contracting a multigraph.

Two usual strategies are applied to hypergraphs, in which the edges (called hyperedges) may connect more than two vertices (KARYPIS et al., 1997). In the first, the hypergraph can be directly contracted by collapsing the matched vertices in set $V_{i}$ into a super-vertex $s V_{i}$ and collapsing and connecting all their incident hyperedges into $s V_{i}$. Fig. $23 \mathrm{~b}$ shows the contraction of the hypergraph in Fig. 23a. Alternatively, a hypergraph $G_{l}$ may be transformed into a graph $G_{l}^{*}$ to which a standard contraction algorithm may be applied. Two traditional abstractions of hypergraphs are common: bipartite-based representations, which map each hyperedge as a new vertex connected to its incident vertices, illustrated in Fig. 23c; and clique-based representations, in which each hyperedge is replaced with simple edges forming a clique connecting its incident vertices, illustrated in Fig. d.

Other studies suggest pruning or filtering the original network as a pre-processing step prior to the contraction. In general, in a first step, unimportant elements, such as isolated and low-degree vertices or low-weight edges, are removed based on specific criteria, and then the 
standard contraction algorithm is employed to build a network approximation or contract some structures, such as cycles or stars. We refer the reader to Liu et al. (2018) for a broad study of filtering and other network summarization strategies.

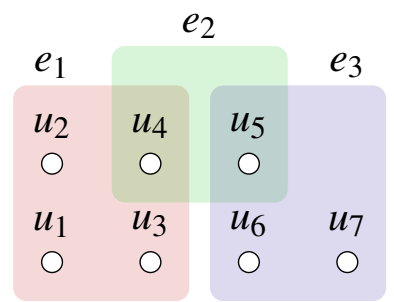

(a) $G_{l}$

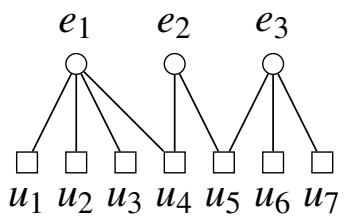

(c) $G_{l}^{*}$

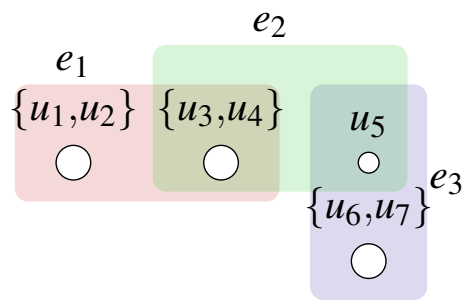

(b) $G_{l+1}$

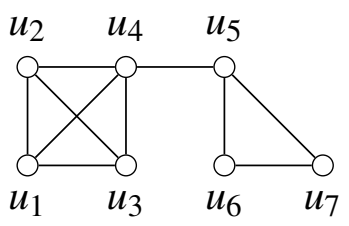

(d) $G_{l}^{*}$

Figure 23 - Result from a direct contraction process of a hypergraph is reported in (b). (c) and d depict two traditional abstraction of the hypergraphy, bipartite-based and clique-based representations, respectively.

\subsection{Uncoarsening}

In the uncoarsening phase, the solution $S_{L}$ found in $G_{l}$ is gradually transferred to the inverse sequence of coarsened networks, i.e. the initial solution is projected and successively refined through the intermediate networks $G_{l-1}, G_{l-2}, \ldots, G_{1}$ up to the original network $G_{0}$. The steps of projection and refinement are detailed next.

\subsubsection{Projection}

How to represent a solution $S_{L}$ is a application-dependent issue. In a community detection problem, $S_{l}$ is constructed from $S_{l+1}$ simply by assigning vertices $\left\{u_{1}, \ldots, u_{k}\right\} \in V_{l}$ to the same set as their sucessor $s V \in V_{l+1}$, therefore it is described as a partitioning of the vertex set into nonempty partitions $C_{k}$ with $\cup C_{k}=S_{L}, C_{k} \subseteq V_{L}$. Each iteration of the uncoarsening phase projects the current solution, obtained in network $G_{l}$, to network $G_{l-1}$ as described in Algorithm 3. The rationale is, for each vertex $u \in V_{l-1}$ in network $G_{l-1}$ (line 2), to obtain its successor vertex $w \in V_{l}$ (line 3) and assign $u$ to its partition (lines 4-5). An implementation may keep the successor vector as a global variable, or it may be handled as a network attribute.

Algorithm 3 can be easily adapted to handle other problems and representations. For example, in overlapping community detection, for each decomposed $s V \in V_{l+1}$, vertices originally in $V_{l}$ are assigned to the same communities of their corresponding super-vertex. In the case of 
coarsened networks with a fuzzy structure, the pertinence of a vertex to a community will be scored in correspondence to its super-vertex. Similarly, in classification problems a vertex will be assigned the same class of its super-vertex. There are scenarios not as straightforward, e.g. to instantiate the algorithm for link prediction, in which $S_{l}$ would be a set of predicted edges $E_{p} \notin E$. In dimensionality reduction problems the projection phase can be suppressed, as the coarsest bipartite network is itself the final solution (VALEJO et al., 2018b).

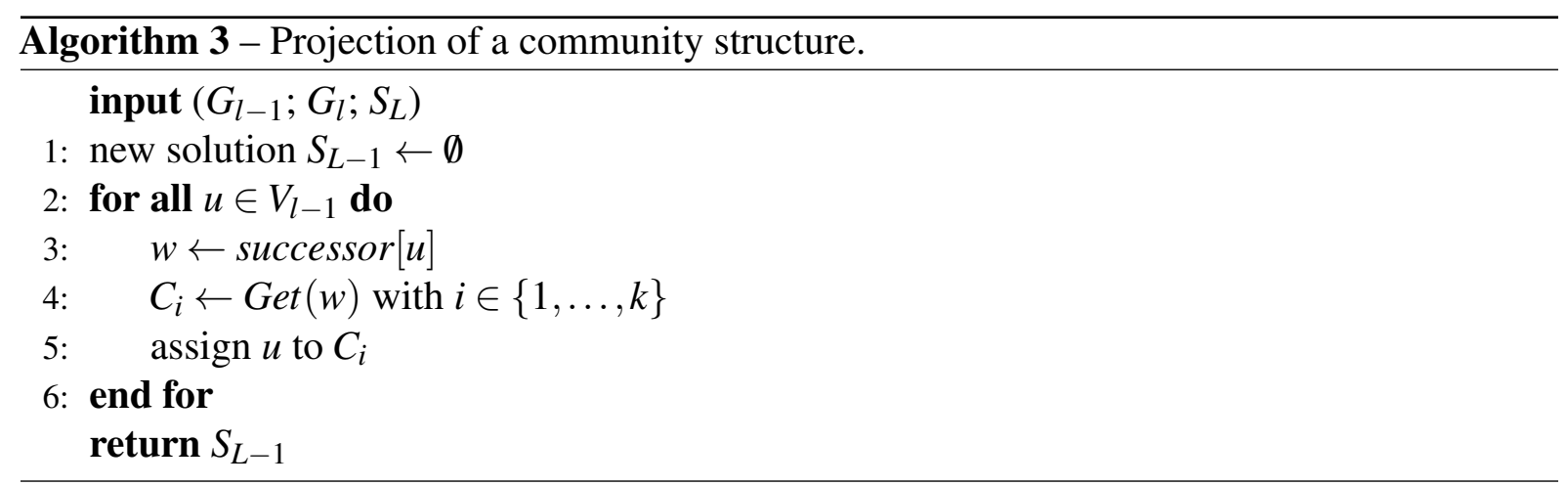

\subsubsection{Refinement}

Even though $S_{l}$ can be an optimal solution in $G_{l}$, this may not be the case of solution $S_{l-1}$ obtained in $G_{l-1}$. Consider, for example, a partitioning problem with the minimum cut criterion. Fig. 24a shows the solution finding phase, where a partitioning algorithm yields the solution $S_{l}=\left\{C_{1}, C_{2}\right\}$ with $C_{1}=\left\{u_{1}, u_{2}, u_{3}, s V\right\}, C_{2}=\left\{u_{6}, u_{7}, u_{8}, u_{9}\right\}$ and $s V=\left\{u_{4}, u_{5}\right\}$ in $G_{l}$. This solution is optimal, since it is not possible to reduce the number of edges between the partitions. Fig. 24b shows the uncoarsening phase, where the original vertices $u_{4}$ and $u_{5}$ are both assigned to the same partition $C_{1}$ of their sucessor $s V$. The solution $S_{l-1}$ projected in $G_{l-1}$ is not optimal, since the cut can be reduced from 3 to 2 by assigning $u_{4}$ to partition $C_{2}$. Fig. $24 \mathrm{c}$ shows the solution refinement.

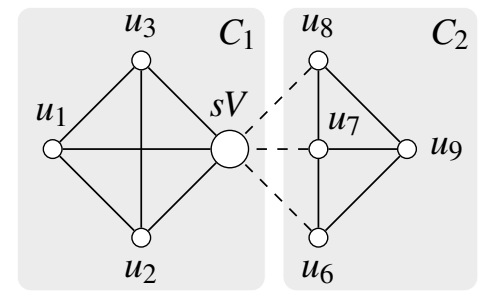

(a) Partitioning of the $G_{l}$

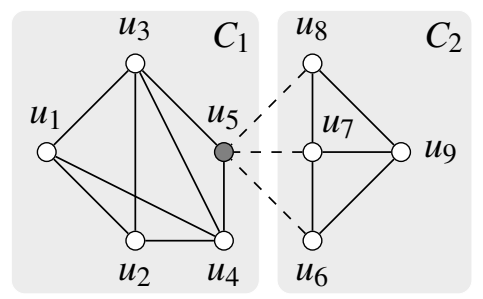

(b) Projection solution to $G_{l-1}$

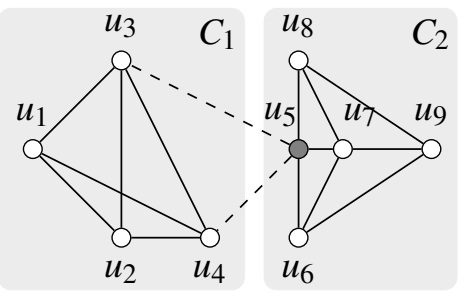

(c) Refinement

Figure 24 - Local minimum problem. In (a) a solution $S_{l}=\left\{C_{1}, C_{2}\right\}$ with $C_{1}=\left\{u_{1}, u_{2}, u_{3}, s V\right\}, C_{2}=$ $\left\{u_{6}, u_{7}, u_{8}, u_{9}\right\}$ and $s V=\left\{u_{4}, u_{5}\right\}$ is found in the coarsest network $G_{l}$. In (b) and (c) $S_{l}$ is projected to $G_{l-1}$ and refined to create the final solution $S_{l-1}=\left\{C_{1}, C_{2}\right\}$, where $C_{1}=$ $\left\{u_{1}, u_{2}, u_{3}, u_{5}\right\}$ and $C_{2}=\left\{u_{4}, u_{6}, u_{7}, u_{8}, u_{9}\right\}$.

Source: Elaborated by the author. 
The coarsening phase is not application-dependent, even if some strategies fit better into certain scenarios. One may, for instance, apply an algorithm such as HEM to network partitioning, image segmentation, network drawing or link prediction problems. The uncoarsening phase, in contrast, is application-dependent; a refinement algorithm designed to improve a partition will not be useful to improve an edge clustering or a link prediction solution. Accordingly, it is possible to apply a refinement heuristics to avoid a local extrema and improve solution quality, i.e., local operations can move the current solution towards a lower cost neighboring solution in the search space.

Most existing refinement algorithms have been conceived for partitioning or community detection problems. They typically operate by moving vertices between partitions, merging two partitions and creating new ones. Many algorithms rely on the Kernighan-Lin (KL) (KERNIGHAN; LIN, 1970) network partitioning heuristics, which performs incremental operations by moving vertices between a bisection in order to reduce its edge-cut count. KL starts with a random bisection and exchanges a selected vertex pair between its partitions if the movement reduces the edge-cut. Departing from a good initial bisection a local minimum can be reached in reasonable time, motivating its adoption as a refinement strategy, i.e. KL is evaluated in the initial solution $S_{l}$ by standard. Although computational complexity is $\mathscr{O}\left(n^{3}\right)$, Fiduccia and Mattheyses (1982) described a linear time implementation relying on appropriate data structures, but limited to two partitions. A generalization to four partitions has been introduced later Suaris and Kedem (1988). Newman (2006) adapted KL to modularity community detection, limited to two partitions; (HENDRICKSON; LELAND, 1995) extended it to handle an arbitrary number of partitions, with complexity $\mathscr{O}(k|E|)$, where $k$ is the number of partitions.

The KK algorithm (KARYPIS; KUMAR, 1998b) is a simplified version of the $k$-way KL refinement algorithm with complexity independent of the number of partitions. Edges that connect vertices in different partitions are called cut-edges and vertices incident to cut-edges are referred to as boundary vertices. In order to reduce the search space, the algorithm only iterates in boundary vertices. The gain in moving a vertex $u$ to a partition $C_{i}$ is given by the sum of all edge-weights of $u$ incident in $C_{i}$. Basically, it moves each boundary vertex $u$ to the partition with the highest gain, as illustrated in Fig. 25. The algorithm is very fast, actually $\mathscr{O}(n)$ in the worst case in which all vertices are boundary vertices.

A refinement strategy called RSim has been introduced by Valejo et al. (2014a) that extends KK incorporating intrinsic social network properties. Rather than considering vertex degrees, it relies on a concept of gain that considers measures of similarity between vertex pairs based on weighted common neighbors, such as Salton and Jaccard. RSim has shown improved performance on social networks but poor accuracy on networks with low clustering coefficients. Another category of refinement algorithms seek to improve a modularity metric (YE; HU; YU, 2008; SCHUETZ; CAFLISCH, 2008a; NOACK; ROTTA, 2009) by iteratively moving individual vertices across partitions, which may also introduce new partitions or merge existing ones. In 
Fig. 26 we illustrate the distinct approaches of a general modularity-based algorithm and of the $\mathrm{KK}$ algorithm in refining a particular vertex.

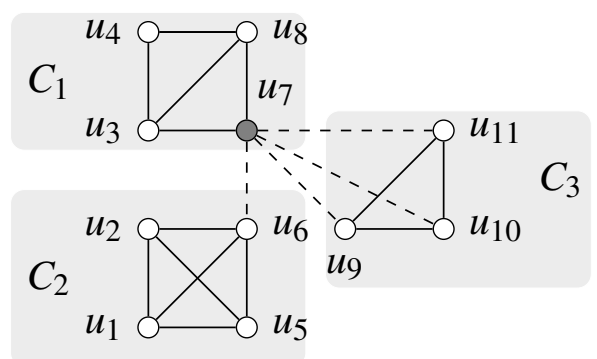

(a) Initial solution $S_{l}$

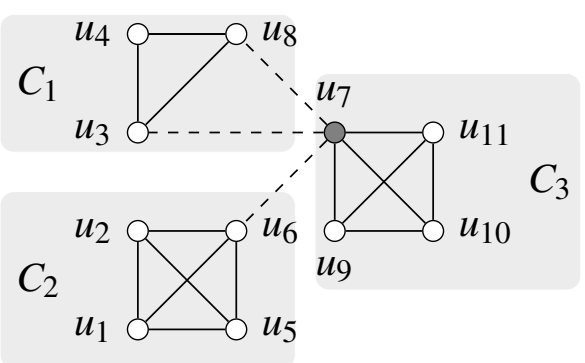

(b) Refined solution $S_{l-1}$

Figure 25 - K-way Kernighan-Lin refinement using a gain function based on the degrees of boundary and non-boundary vertices: (a) shows an initial solution and (b) shows the refined solution $S_{l-1}$, wherein vertex $u_{7}$ has been moved to $C_{3}$.

Source: Elaborated by the author.

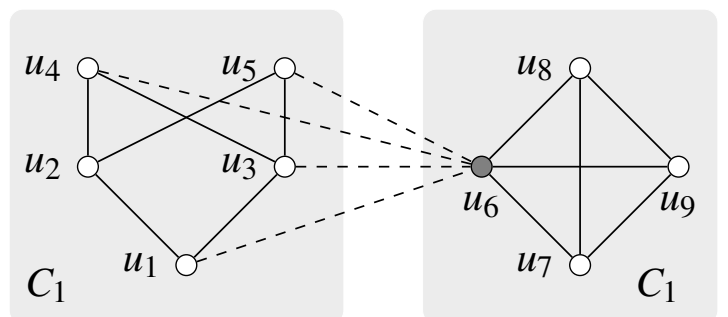

(a) Modularity-based

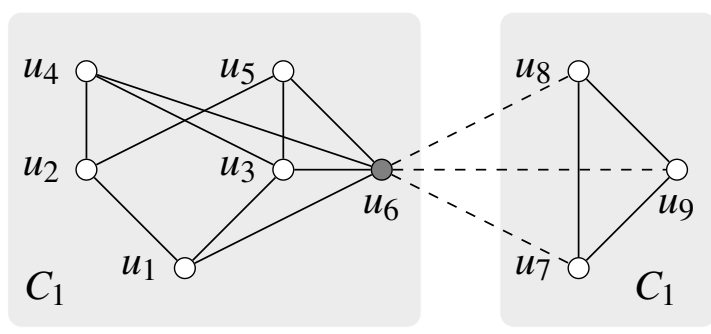

(b) KK

Figure 26 - Difference between a modularity-based algorithm and the KK algorithm in refining the assignment of the vertex $u_{6}$. (a) A modularity-based algorithm keeps $u_{6}$ in $C_{2}$, yielding a global modularity of 0.2500 and a cut of 4 . (b) the KK algorithm moves $u_{6}$ to $C_{1}$, yielding a global modularity of 0.21676 and a cut of 3 .

Source: Elaborated by the author.

The refinement algorithms are tied to the solution definitions imposed by the target algorithms, therefore, a perspective that must be considered in a discussion of their advantages and limitations. A modularity-based algorithm, for instance, may be adequate in scenarios where preserving he community organization is important. However, it may not be the best choice if the solution derives from a min-cut formulation, as illustrated in Figure 26, where the KK algorithm may be a better choice for refinement. The uncoarsening phase is thus application-dependent by definition. In our experience, an ad-hoc refinement choice not guided by specific criteria may lead to inconsistent final solutions. Most studies present refinement algorithms considering a specific scenario, or in combination with a particular target algorithm. 


\subsection{Applications}

Considering the framework and underlying concepts presented along this text, this section describes case studies illustrating how certain classical and representative machine learning tasks may be addressed from a multilevel perspective.

\subsubsection{Visualization}

Network visualization deals with the problem of creating visual representations of networked data that support user-driven exploratory investigation. Visualizations are expected to have reduced visual complexity and be aesthetically pleasing, in order to assist users in gaining insight on the global and local behavior of data.

A multilevel visualization can be summarized as follows: initially, the network is successively coarsened until reaching a sufficiently small network. In a second step the visual mapping is performed drawing the coarser network using a layout algorithm. A user may interact with this representation in a visual data analysis process. This may be supported by on-demand expansion of the visual representation, supported by the multilevel hierarchy, i.e., the user interacts to expand regions locally at further levels of detail, as needed, and navigate back and forth through the sequence of coarsened networks for more or less detail.

An illustrative example is presented of a large bipartite network obtained from the SuitSparse Matrix Collection ${ }^{12}$ using the approach discussed by Valejo et al. (2018b). Rajat is a nonsymmetric circuit simulation matrix representing 6,765 individual resistors. The data matrix is modeled as a bipartite network, in which rows depict the first layer, columns depict the second layer and non-zero cells define the edges and their weights. Figure 27 a shows force-directed layouts of the original Rajat bipartite network and Figures 27b and 27c illustrates layouts of the networks obtained at two levels of coarsening, respectively with $|V|=2,000$ and $|V|=200$. The original network has a giant module surrounded by small modules; the coarsened networks in Figures $27 \mathrm{~b}$ and $27 \mathrm{c}$ reduce the information displayed favoring the distinction of the inter and intra-community edges, as well as of vertex types and neighboring edges. The coarsened models, albeit losing detail, reveal topological patterns hidden, or blurred, in the full network layout.

\subsubsection{Community detection}

Community detection is the benchmark problem for multilevel methods, in which the goal is to split the network vertices into disjoint (potentially overlapping) groups, called communities, seeking to minimize the occurrence of edges between distinct communities (FORTUNATO, 2010). Empirical results from community detection with multilevel strategies reveal a considerable speed-up of the target algorithms with limited loss in solution quality (VALEJO et al., 2018b).

$12<\mathrm{https}: / /$ sparse.tamu.edu/> 


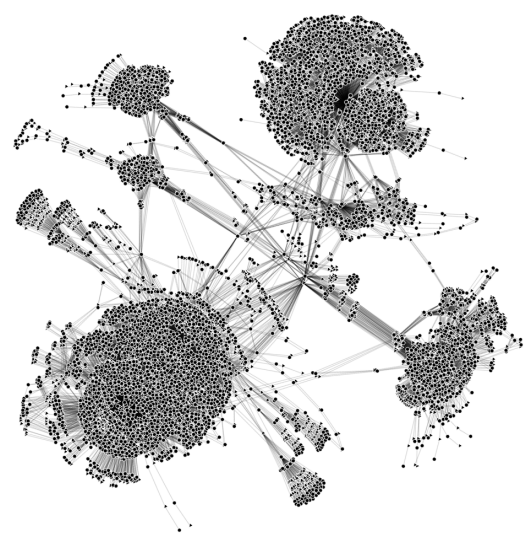

(a) Original network

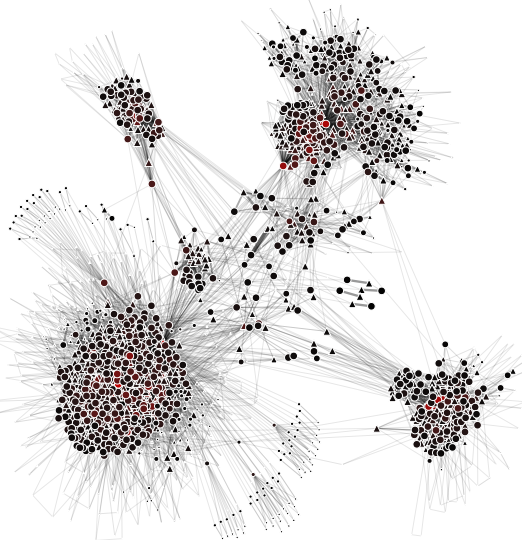

(b) Coarsened network $G_{L-1}$

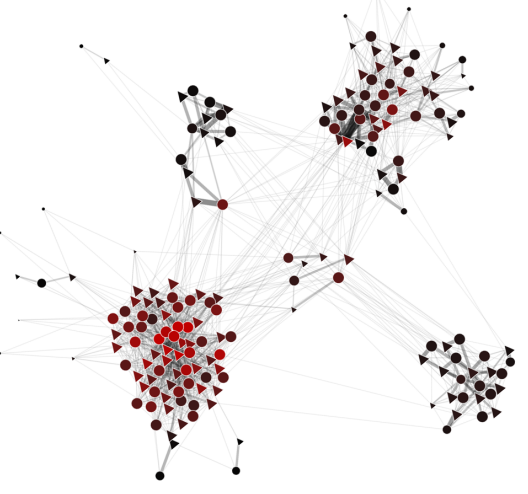

(c) Coarsest network $G_{L}$

Figure 27 - Rajat bipartite network with 13,530 vertices and 43.180 edges: (a) shows the original network; (b) and (c) illustrate it at two levels of coarsening, with $|V|=2,000$ and $|V|=300$, respectively. Graph markers representing the vertex type with different shapes, triangle and circle, and super-vertices are drawn using a black-to-red color scale, in which red vertices denote highweight super-vertices. The layout has been computed with the force-directed algorithm (FRUCHTERMAN; REINGOLD, 1991).

It is straightforward to employ a coarsening strategy directly as a community detection algorithm (NOACK; ROTTA, 2009), just by considering super-vertices as communities and projecting this solution back to the original network. Considering a hypothetical example, Fig. 28 illustrates the strategy applied with a coarsening algorithm based on triangle matching, as in Gupta (1997), Oliveira and Seok (2007a), Kang et al. (2015b), Kang et al. (2015a). First, each triangle in the coarsened network is contracted into a super-vertex, level by level, until no triangles remain, Figs. 28a-28c. The super-vertices are then assigned a unique label, Fig 28d, and labels are projected back to their corresponding predecessor-vertices at each coarsening level, up to the original network 28a-28f. Vertices assigned the same label define the resulting communities. 


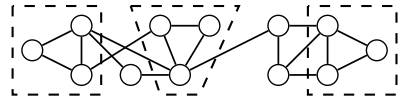

(a) $G_{0}$

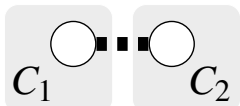

(d) $G_{2}$

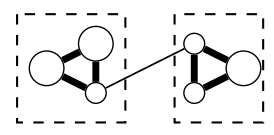

(b) $G_{1}$

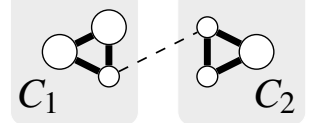

(e) $G_{1}$

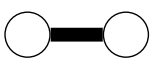

(c) $G_{2}$

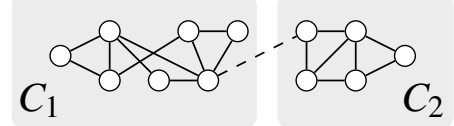

(f) $G_{0}$

Figure 28 - Multilevel community detection based on triangle matching.

\subsubsection{Dimensionality reduction}

Machine learning tasks on high-dimensional data are often preceded by a dimension reduction step, as the dimensionality of the representation space has potential negative impacts on the time and accuracy of the algorithms, known as the "dimensionality curse". Although the multilevel method has been under-explored in this context, some authors report applications on dimension reduction.

Sakellaridi, Fang and Saad (2008), and later Fang, Sakellaridi and Saad (2010), employed the standard multilevel framework to optimize an accurate high-cost dimension reduction algorithm. They execute the target algorithm on the coarsest network to obtain a reduced attribute set and this initial solution is projected and refined back to the original network.

Valejo et al. (2018b) introduced an intuitive approach modeling the data matrix as a bipartite network, so that objects (rows) and attributes (columns) are mapped to the network layers and the non-zero entries in the matrix correspond to the edge weights, see Fig. 29. The underlying rationale is to contract the attribute layer while preserving the object layer. As the bipartite network is represented as a bi-adjacency matrix $A_{r \times s}$, where $r=\left|V_{1}\right|, s=\left|V_{2}\right|$ and $A_{u, v}=w(u, v)$ if $(u, v) \in E$, dimensionality reduction seeks to create a lower dimensional matrix

$A_{r^{\prime} \times s^{\prime}}^{\prime}$, with $r^{\prime}=r$ and $s^{\prime} \ll s$. Fig. 29a depicts the original matrix and corresponding bipartite network, $X_{0}$ and $G_{0}$, respectively, and the matching $M=\left\{\left\{s_{1}, s_{2}\right\},\left\{s_{2}, s_{3}\right\}\right\}$. Fig. 29b shows the coarsened network $G_{1}$ and its reduced bi-adjacency matrix representation.

\subsubsection{Graph coloring}

The graph coloring problem consists of assigning a minimum number of colors to the vertex set ensuring adjacent vertices are assigned different colors. It has application in many tasks, e.g., map coloring, in which adjacent cities (or states) should not be assigned the same color; bipartite graph verification, that verifies whether an input graph is bipartite; and resource allocation, to distribute resources minimizing the costs or maximizing the returns.

A general multilevel solution is described in Walshaw (2001b), Walshaw (2004), Walshaw 


\begin{tabular}{|c|c|c|c|}
\hline & $s_{1}$ & $s_{2}$ & $s_{3}$ \\
\hline$r_{1}$ & 3 & 0 & 0 \\
\hline 2 & 2 & 3 & 0 \\
\hline 3 & 0 & 2 & 0 \\
\hline & 0 & 1 & 4 \\
\hline & 0 & 0 & 3 \\
\hline
\end{tabular}

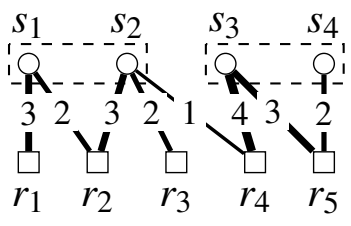

(a)

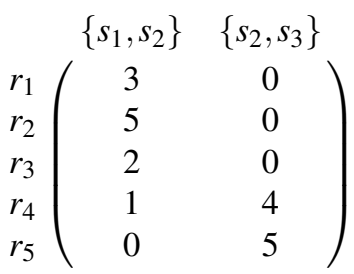

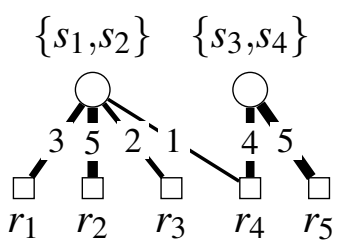

(b)

Figure 29 - Multilevel dimensionality reduction.

(2008b), in which matches are allowed between non-adjacent vertices. In this strategy, illustrated in Fig. 30, a vertex $u$ is only allowed to match with its unmatched neighbors $\left\{v \mid v \in \Gamma_{2}(u)\right\}$. The underlying rationale is that if a super-vertex $s V=\{u, v\} \in G_{l}$ has been assigned a color, the same color can be assigned to its predecessor-vertices $\{u, v\} \in G_{l-1}$ without coloring conflicts. Figs. 30a-30c show the coarsening process, i.e., the hierarchy $H_{C}=\left\{G_{0}, G_{1}, G_{2}\right\}$. The coarsening continues until the coarser network consists of a single $k$-clique or disconnected $k$-cliques. Finally, each vertex in a clique is assigned a different color and the solution is projected back to the original network, as illustrated in Figs. 30c and 30d.

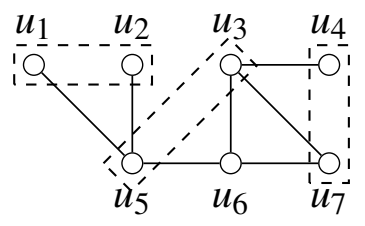

(a) $G_{0}$

$$
\left\{u_{1}, u_{2}, u_{6}\right\}
$$

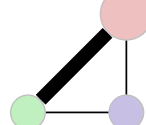

$\left\{u_{3}, u_{5}\left\{u_{4}, u_{7}\right\}\right.$

(c) $G_{2}$

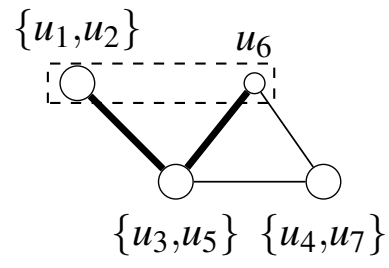

(b) $G_{1}$

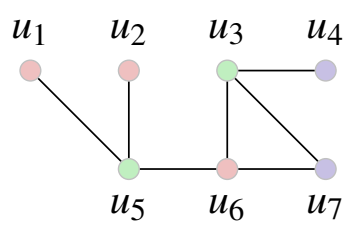

(d) $G_{2}$

Figure 30 - Multilevel graph coloring: a shows the original network $G_{0}$ and the first vertex-selection matching process with $M=\left\{\left\{u_{1}, u_{2}\right\},\left\{u_{3}, u_{5}\right\},\left\{u_{4}, u_{7}\right\}\right\}$; (b) shows the first level coarsening $G_{1}$; (c) shows the coarsened network $G_{2}$ and the initial solution; and (c) the projected solution. 


\subsection{Concluding Remarks}

The multilevel method is a general problem-solving strategy with huge applicability. Initially motivated by efforts to solve partitioning problems on large graphs, it has found application in a range of network-based problems. The diversity of problem domains and ramifications therein has not favored systematic descriptions of the general method nor the adoption of a consistent terminology. Terms are often employed in the literature inconsistently, or mixed with domain jargon; the multilevel phases are not always explicitly described as so, e.g. the uncoarsening phase is sometimes omitted from the discussion. Indeed, many application-focused papers introduce the theoretical background required to understanding their particular context and the general strategy remains implicit. As a result, newcomers struggle to gather a consistent background on the field. We believe this scenario contributes to undermine the perception of the method's potential to solve other types of network-based problems beyond partitioning, in particular general data mining tasks defined in $k$-partite and heterogeneous networks.

This survey results from our effort to integrate and systematize the current knowledge on multilevel strategies applied to complex networks. We provide both an overview and a coherent organization of the literature and related terminology, addressing the key concepts of multilevel optimization under a unified conceptual framework. Existing algorithms for each multilevel phase have been reviewed, including a critical analysis of matching algorithms and their relative advantages and drawbacks. The algorithms have been categorized according to their underlying strategy, namely edge-selection, vertex-selection, or cluster-selection.

We discuss several conceptual and practical issues related to the coarsening and uncoarsening phases in order to enhance understanding of the method. The choice of matching strategy is critical, since there is a plethora of algorithms for this purpose and they affect the network topology in different ways. Modularity-based algorithms, as proposed by Noack and Rotta (2009), tend to create regular and sparse networks and the selection following the concept of the groups of "densely" connected vertices and sparsely connected to vertices outside the group. Isospectral reduction strategy, as presented in Bunimovich and Webb (2012), reduces a network while preserving its spectral properties, maintaining the eigenvalues, including multiplicities of its adjacency matrix. Therefore, the choice of the coarsening strategy should consider a suitable trade-off between accuracy, execution times, network topology and matching criteria. According to our opinion, this question is the most critical shortcomings within the literature, therefore, address this issue empirically would be helpful and a promising future work.

Similarly, certain scenarios could benefit from exploiting variations of the standard network contraction strategy, e.g., would it be possible to create coarsened networks from overlapping communities of vertices? Or, what would be the effect of adopting alternative ways of deriving super-vertex or super-edge weights, or other properties?

As an additional contribution, this survey provides a systematic mapping of the liter- 
ature on multilevel methods in complex networks. We conducted a meta-analysis of selected representative publications, and organized the data collected to convey a broad overview of the relevant contributions and a historical underpinning of the field. The literature reveals a remarkable effort towards employing multilevel optimization in multiple problems defined in complex networks, with a historical emphasis on community detection and related problems. Nonetheless, there is still much potential to address a wider class of data mining tasks defined in bipartite or heterogeneous networks, such as classification and link prediction.

On the link prediction problem, Lü and Zhou (2011) (also addressed in temporal networks (MA; SUN; QIN, 2017)) reported three major challenges: accuracy, complexity and robustness. Distance-based similarity indexes based on global network information yield accurate and robust results at a high cost. Similarly, the high computational cost prevents handling real-world text collections with recent classification algorithms that rely on bipartite networks, such as TPBG (Transductive Propagation in Bipartite Graph) (FALEIROS; ROSSI; LOPES, 2017). In both cases multilevel strategies offer a promising path towards developing solutions capable of handling larger scale networks.

Indeed, bipartite, $k$-partite (or multi-layer) networks often arise in connection with studies of social or biological phenomena (BURGOS et al., 2008), as well as of recommendation systems (KUNEGIS; LUCA; ALBAYRAK, 2010). Recent efforts have addressed community detection (MA; DONG; WANG, 2018) and dimension reduction, or introduced a general theoretical framework of the multilevel method for bipartite networks (VALEJO et al., 2017; VALEJO et al., 2018a; VALEJO et al., 2018b). Yet, the importance of the problems is still to be matched by an offer of appropriate multilevel optimization solutions.

Heterogeneous networks describe multiple types of vertices and possibly multiple types of relationships (PHAM; DO, 2019). Examples include document-word networks indicating associations between document-word, word-word, and document-document; or describing relations between words, documents and authors. Such networks are common in network science and applications (SHI et al., 2017a), e.g. in the context of link mining and analysis, social network analysis, hypertext, web mining and graph mining. While their complex nature poses significant challenges, they are extremely rich in structure and semantics. Extending multilevel optimization to problems defined in such networks is certainly a promising research topic.

Our survey compiles a unified reference to the literature, organizing the contributions under a theoretical and conceptual framework. It also includes a critical assessment of the many strategies and choices available to implement a multilevel solution. Hopefully, it can contribute to reduce the effort required from newcomers to grasp the current state-of-the-art in multilevel optimization, inspire new directions of investigation and pave the development of novel applications. 


\section{Appendix}

\section{A Glossary of Terms}

The glossary compiles the most relevant terms employed in describing the multilevel definitions and related theory, as well as the basic concepts of complex networks and community structure.

MO : Multilevel Optimization (aka. MS: Multilevel Strategy).

Coarsening : The first phase of the MO: the initial network is reduced in to a sequence of coarsened networks.

Coarsened network : Small network approximation in a specific level.

Coarsest network : The most reduced network at the deepest level (or last level).

Collapsing : Union of matched vertices

Contraction : Build a reduced network from groups of vertices

Uncoarsening : The third phase of the MO: the initial solution is projected back (and refined) to the original network.

Refinement : Iterative solution improvement process.

Target algorithm : Expensive (or high-cost) algorithm used in the solution finding phase

Solution finding : The second phase of the MO: the initial solution is computed.

Original network : The initial problem instance (aka. initial network or original problem).

Initial solution : Solution obtained in the coarsest network.

Final solution : Solution obtained (or projected back) in the original network.

Refined solution : Improved solution in a specific level.

Matching : A partition of $V$ into a set of $K$ non-empty and disjoint subsets.

Matched vertex : A vertex $u \in V_{i}$ such that $V_{i} \in M$.

Unmatched vertex : A vertex $u \nexists V_{i} \in M \mid u \in V_{i}$.

Singleton : Unmatched vertex

Super-vertices : Set of matched vertices.

Successor (super-vertex) : Super-vertices are called a successors of its originating vertices.

Predecessors (vertex) : Vertices $\left\{u_{1}, \ldots, u_{n}\right\} \in V_{l}$ collapsed into a super-vertex $s V_{i} \in V_{l+1}$.

Super-edges : Set of edges incident to matched vertices.

Successor (super-edge) : Super-edges are called a successors of its originating edges.

Predecessors (edges) : Edges $\left\{e_{1}, \ldots, e_{n}\right\} \in E_{l}$ collapsed into a super-edge $s E_{i} \in E_{l+1}$.

Successor-network : A network $G_{l+1}$ is a sucessor of the network $G_{l}$.

Predecessor-network : A network $G_{l}$ is a predecessor of the network $G_{l+1}$.

Edgeless : Network with no edges or zero edges.

Clique : Fully connected subnetwork (or complete subnetwork).

$k$-clique : Clique of cardinality $k$.

Subnetwork : Vertex set of $V$. 
Hub : A vertex with high degree (or strength).

Isolated vertex : Vertex with no edge.

Leaf vertex : Vertex with one edge.

ESM : Edge-selection matching strategy.

VSM : Vertex-selection matching strategy.

CSM : Cluster-selection matching strategy.

Boundary vertex : Vertex $u$ connected with two or more communities.

Non-boundary vertex : Vertex $u$ connected with only a community.

| | : Cardinality of a set.

$\mathbb{R}^{*}:$ Set of positive real numbers.

$\mathbb{R}:$ Set of real numbers.

$\mathbb{N}$ : Set of natural numbers.

$G$ : Network.

$V:$ Set of vertices.

$V_{i}$ : Non-empty and disjoint subset of $V$.

$u$ : Vertex.

$v:$ Vertex.

$E$ : Set of edges.

$e_{i}$ : Edge $i$.

$\sigma:$ Set of vertex weights.

$\sigma(V)$ : Total vertex weight.

$\sigma\left(v_{j}\right):$ Weight of a vertex $v_{j}$.

$\omega$ : Set of edge weights.

$\omega(E)$ : Total edge weight.

$\omega(u, v)$ : Edge weight between (aka. $\omega_{i}$ associated with a corresponding edge $\left.e_{i}=(u, v)\right)$.

$\Gamma_{h}(v): H$-hop neighborhood of a vertex $v$.

$C$ : Clustering or community structure.

$C_{i}$ : Non-empty and disjoint subset of $C$.

$C(u)$ : Cluster $C_{i}$ that contains the vertex $u$.

$C\left(C_{i}\right)$ : Vertex set in $C_{i}$.

$C_{i}^{i n}$ : Sum of the weights of the edges ending in $C_{i}$.

$C_{i}^{\text {out }}$ : Sum of the weights of the edges that connect vertices in $C_{i}$ to vertices in other clusters.

$C_{i}-\{v\}$ : Cluster $C_{i}$ with the vertex $u$ removed.

$C_{j}+\{v\}$ : Cluster $C_{i}$ with the vertex $u$ added.

$d_{i}$ : Degree of vertex $i$.

$d_{G}:$ Degree of the graph $G$

$d_{u \rightarrow C_{i}}$ : Number of neighbors of $u$ that are in $C_{i}$. 


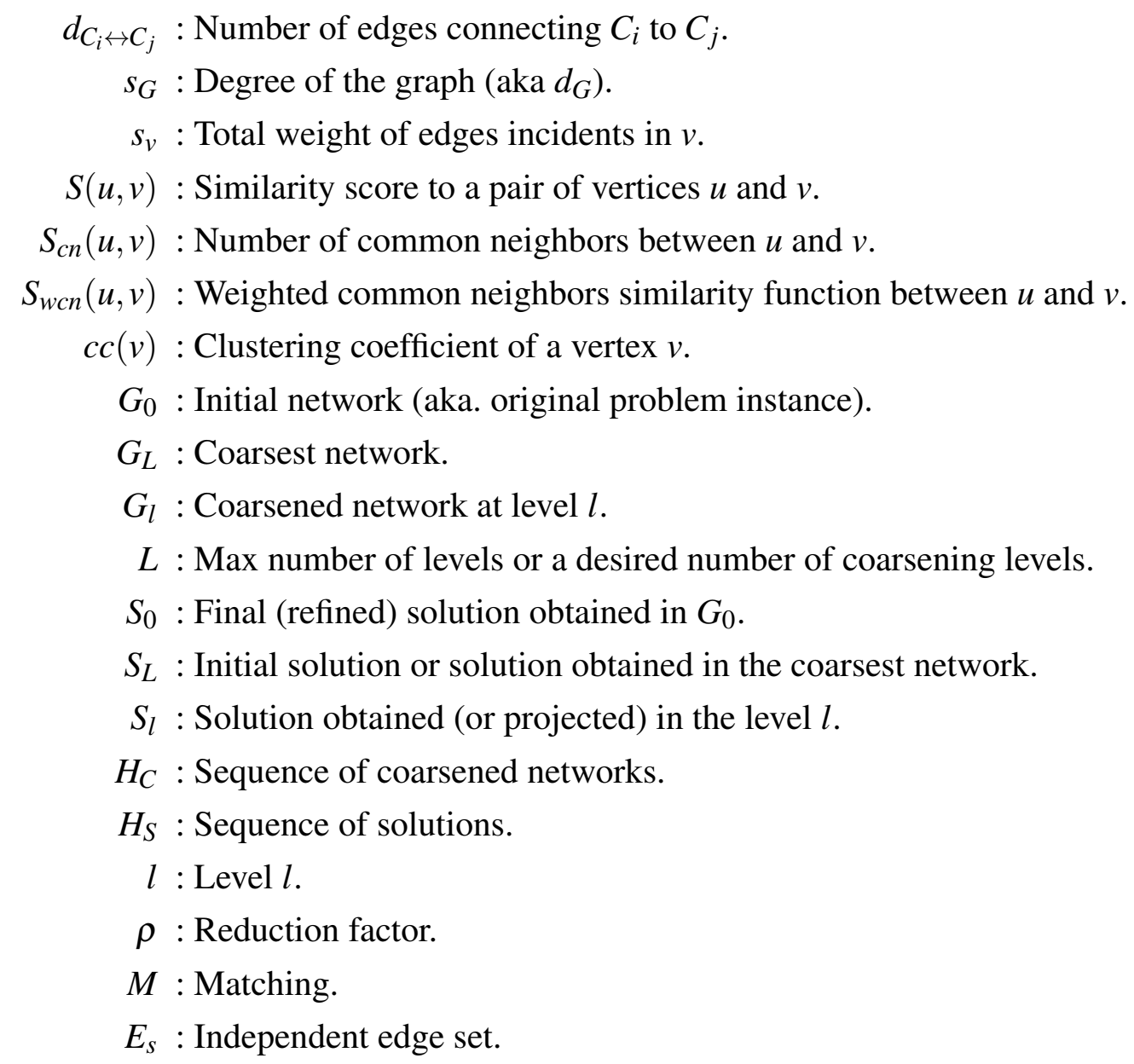

\section{B Supplemental material}

This appendix contains supplemental information relative to the matching and refinement algorithms reviewed in the survey.

\section{B.1 Matching algorithms RM, HEM, LEM, MHEM, NCN, ANCN, WANCN and HCM}

All matching algorithms discussed, namely RM, HEM, LEM, MHEM, NCN, ANCN, WANCN and HCM, follow a similar structure depicted in Algorithm 4. They differ essentially in the restriction implemented in Line 5. In line 4 a vertex is selected in some established order, e.g., random order in RM, ascending degree order in SRM. In line 5 vertex $u$ is matched with one of its unmatched neighbors $v$ based on some restriction, e.g., in RM $v$ is randomly selected and in NCN it is the vertex with the maximum number of common neighbors.

\section{B.2 Greedy heavy edge matching algorithm GHEM}

In GHEM edges are sorted by descending weight and then visited iteratively as expressed in Algorithm 5. For each visited edge $(u, v)$, if neither $u$ nor $v$ are matched yet, the pair $V_{i}=\{u, v\}$ 


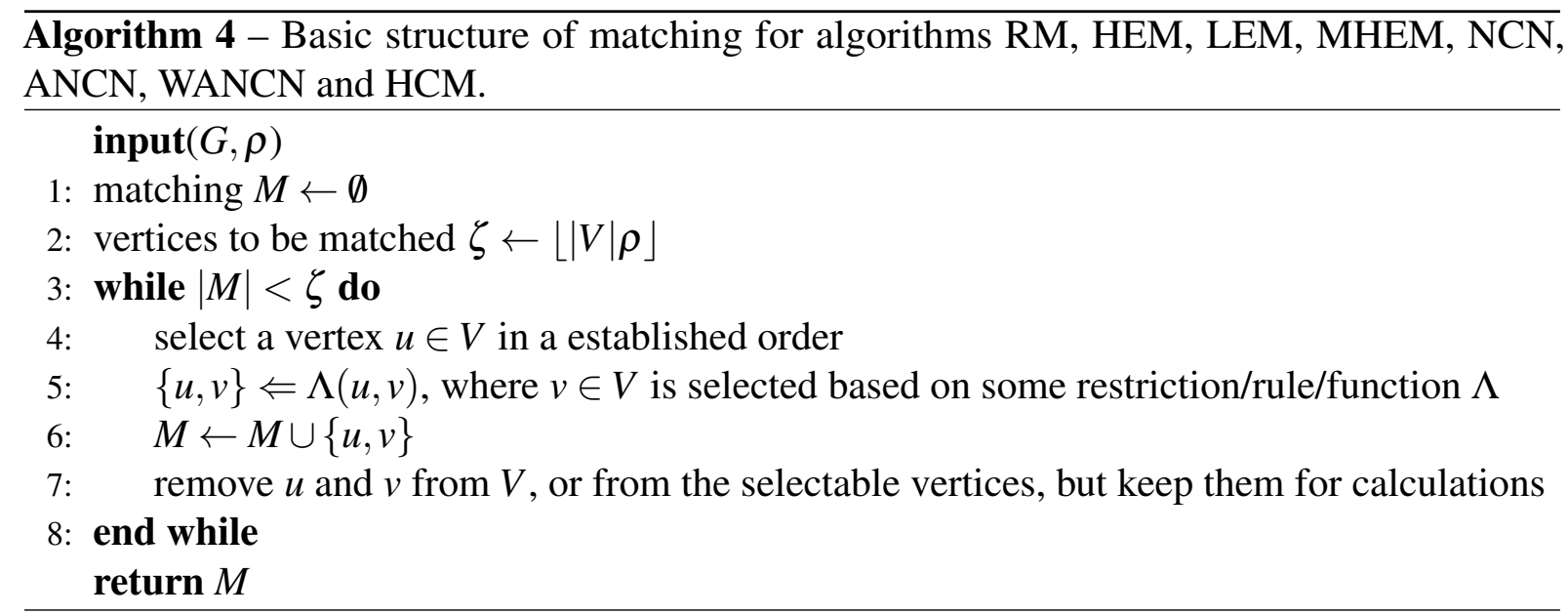

is included in $M$. The algorithm is near linear-time, and although slightly more expensive than other methods it is more robust.

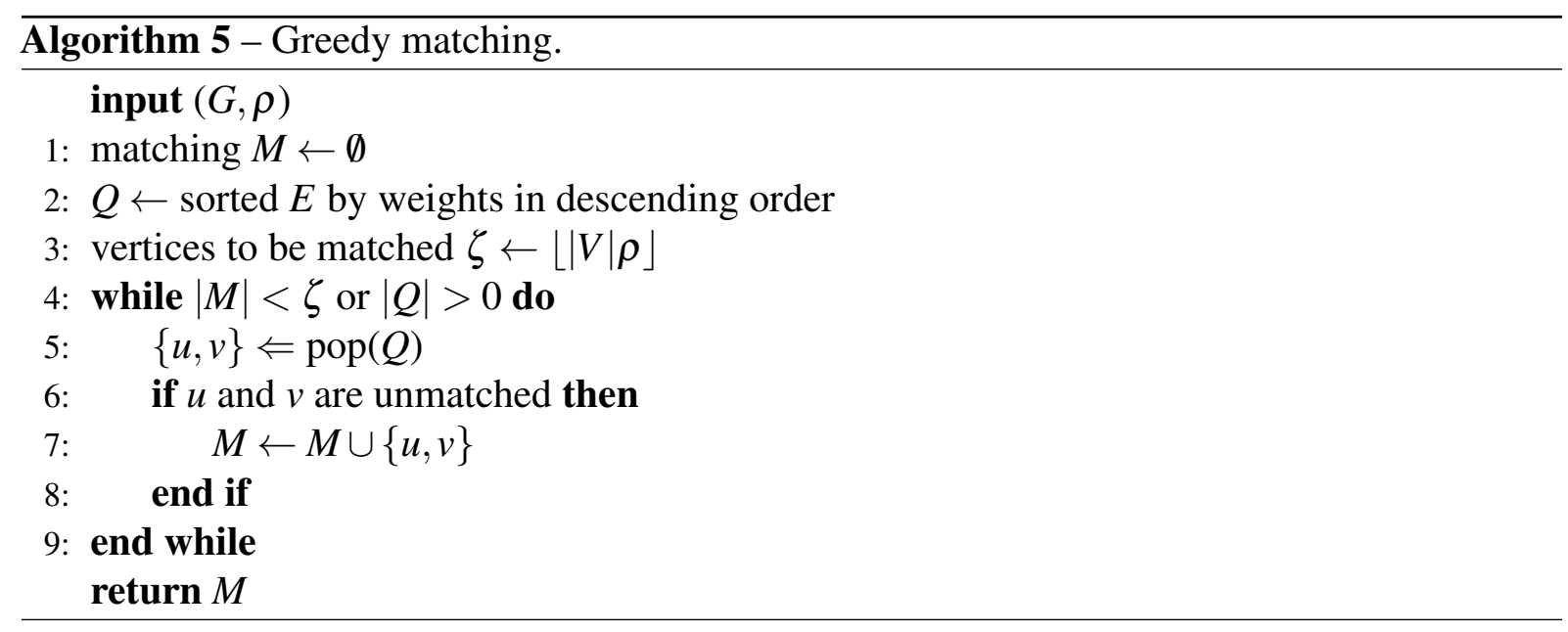

\section{B.3 Path growing matching algorithms}

Algorithm 6 summarizes the basic structure of a path growing strategy. It starts with a randomized vertex $u$ (i.e., a path of zero-length or no edges). Next, it extends the current path and selects the heaviest edge $(u, v)$ currently available. Selected edges are alternately inserted into $M_{1}$ and $M_{2}$. To ensure paths with disjoint vertices, all other edges incident to $u$ are disabled. It finishes when all vertices belong to some path, returning the path with heaviest edge weight.

\section{B.4 Edge-rating algorithms}

Sanders and Schulz (2011) and Holtgrewe, Sanders and Schulz (2010) presented a network partitioning software called Karlsruhe Fast Flow Partitioner (KaFFPa) that employs two simple and efficient edge-rating functions:

$$
\operatorname{expansion}^{2}(u, v)=\frac{\omega(u, v)^{2}}{\sigma(u) \sigma(v)}
$$




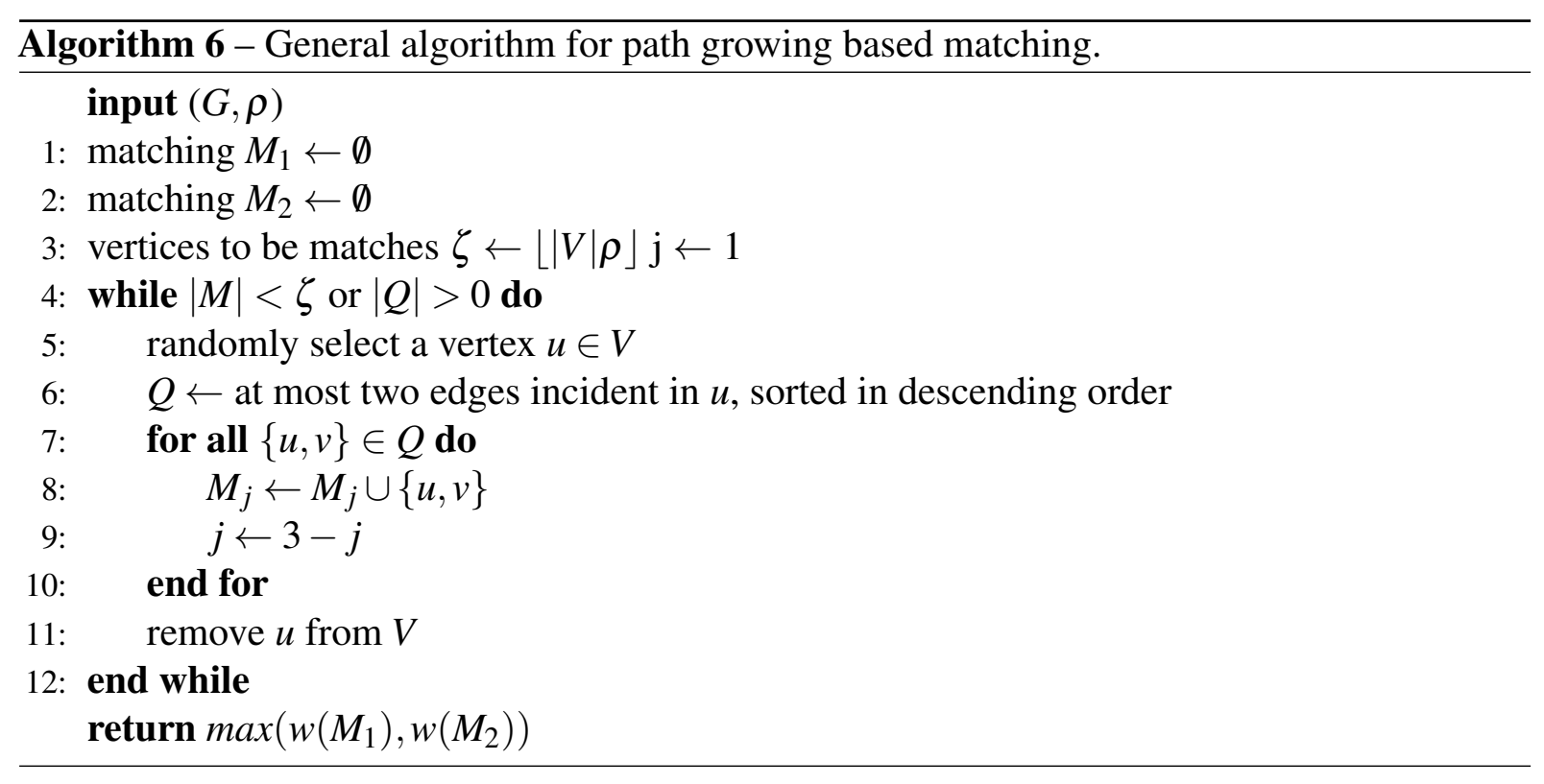

and:

$$
\operatorname{innerOuter}(u, v)=\frac{\omega(u, v)}{\operatorname{Out}(v)+\operatorname{Out}(u)-2 \omega(u, v)}
$$

where:

$$
\operatorname{Out}(u)=\sum_{v \in \Gamma_{1}(u)} \omega(u, v)
$$

Chen and Safro (2011) and Safro, Sanders and Schulz (2012) introduced a more sophisticated edge-rating function based on spectral theory. Let be $L=D-W$ the network laplacian matrix, wherein $W$ is the weighted adjacency matrix and $\mathrm{D}$ is a diagonal matrix with entries $D_{i i}=\sum_{j} \omega(i, j)$. The volume-normalized version is denoted by $\widetilde{L}=\widetilde{D}-\widetilde{W}$ and the volume-normalized edge-weight is $\widetilde{\omega}(i, j)=\omega(i, j) / \sqrt{(} \sigma(i) \sigma(j))$. An iteration matrix $H$ for Jacobi over-relaxation (with $\alpha=1 / 2$, well-known as a lazy random-walk matrix) is defined as $H=(1-\alpha) I+\alpha \widetilde{D}^{-1} \widetilde{W}$, wherein $0 \leq \alpha \leq 1$. The authors defined the algebraic distance $p_{u, v}$ as:

$$
p_{u, v}=\left[\sum_{r=1}^{R}\left|X_{u}^{(k, r)}-X_{v}^{(k, r)}\right|^{2}\right]^{\frac{1}{2}}
$$

whereas $X^{(k, r)}=H^{k} X^{0, r}$ is a relaxed randomly initialized test vector (i.e., $X^{(0, r)}$ is a random vector sampled over $[-1 / 2,1 / 2]), R$ is the number of test vectors, and $k$ is the number of iterations. Authors considered $\alpha=0.5, R=5$, and $k=20$ as appropriate values in the general case. The edge-rating function $E R(u, v)$ is defined as:

$$
E R(u, v)=\frac{1}{p_{u, v}} \operatorname{expansion}^{2}(u, v)
$$


Other studies employed edge-rating functions with algebraic formulations (SAFRO; RON; BRANDT, 2006; BRANNICK et al., 2013; GLANTZ; MEYERHENKE; SCHULZ, 2014). In general, it is possible to integrate the edge-rating function with any other iteration strategy.

Following an edge-rating strategy, Purohit et al. (2014) proposed the CoarseNet matching algorithm that builds a succinct network representation while preserving its diffusive properties. The most important diffusion features of a network are captured by the first eigenvalue of its adjacency matrix. Therefore, a coarser version of an input network can be considered representative if its first eigenvalue is similar to the original. The algorithm first rewrites the edges appropriately based on a score $s(u, v)$, derived using matrix perturbation theory. If the estimator $s(u, v) \approx 0$ the edge $(u, v)$ does not play a significant role in the diffusion through the network and can be matched. Then, the algorithm successively selects adjacent vertex pairs which minimize the change in the first eigenvalue.

Other edge-selection methods for matching are based on non-negative matrix factorization (NMF). NMF aims to (approximately) factorize a non-negative data matrix $X$ as a product of two low-rank matrices $W$ and $H$ sych that $X \approx W \cdot H$, where $W, H \in \mathbb{R}_{+}$. If $X$ has dimensions $n \times m$ and $k$ is a user-defined parameter for rank approximation, matrices $W$ and $H$ must be, respectively, $n \times k$ and $k \times m$, and:

$$
x_{i} \approx \sum_{j=1}^{k} w_{j} \cdot H_{j i}
$$

wherein $x_{i}$ is the $i$-th column of $X, w_{j}$ the $j$-th column of $W$ and $H_{j i}$ is the entry $(j, i)$ of $H$. The parameter $k$ often set as $k \ll \min (m, n)$, since the aim is to reduce the size of the dimension. The factorization itself is usually posed as an optimization problem and is computed as the minimum of the expression:

$$
\min _{W, H \geq 0}\|X-W H\|_{F}^{2}
$$

wherein $\|X\|_{F}^{2}=\sum_{i j} X_{i j}^{2}$ is the squared Frobenius norm of matrix $X$. In general, it can be solved using a direct optimization or an alternating optimization strategy that partitions the NMF problem into two subproblems for matrices $W$ and $H$, i.e., at each iteration, the problem is solved for one of the matrices while the other is held fixed. Algorithm 7 shows an alternative general optimization strategy, which can be faster than direct optimization and better at avoiding a local maximum.

The classical NMF formulation, as described in Equation 2.7, does not consider neighborhood relations (the geometric structure), which may imply in unsatisfactory performance e.g. in clustering tasks. Cai et al. (2011) proposed Graph Regularized Non-negative Matrix Factoriza- 


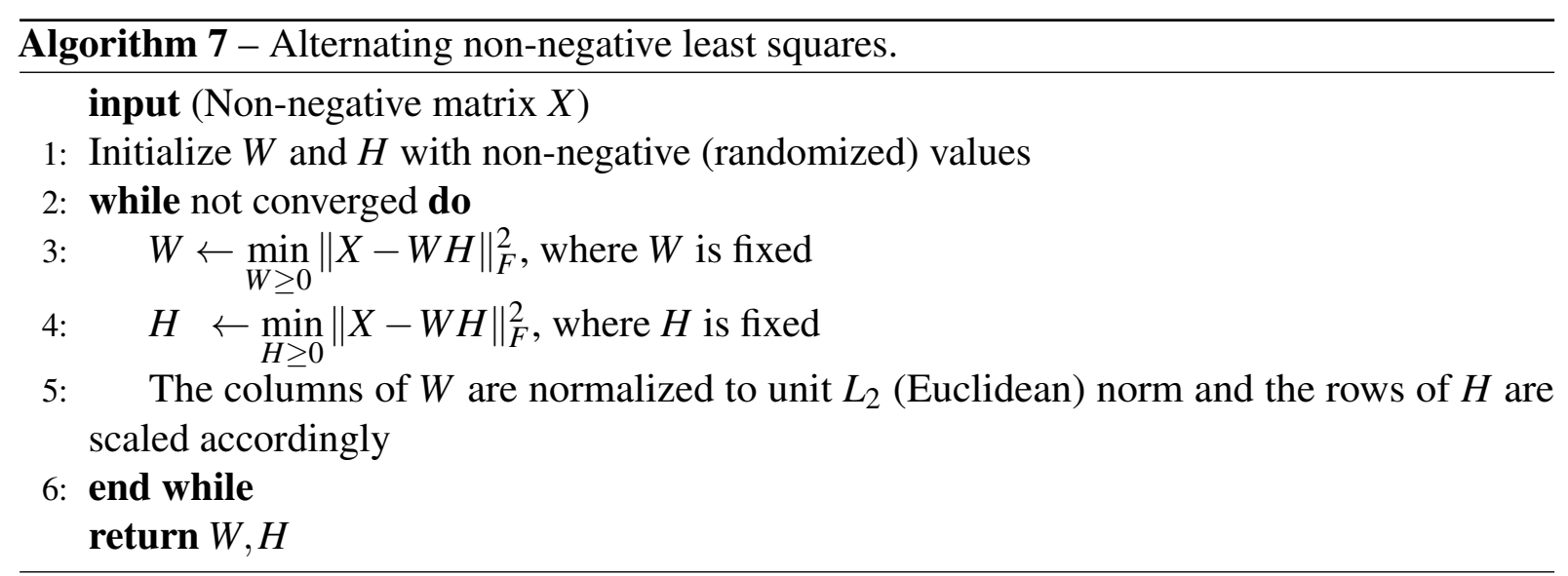

tion (GNMF) that preserves the geometric structure of the dataset, i.e. keeps the neighborhood relations. GNMF follows the cost function:

$$
\min _{W, H \geq 0}\left(\|X-W H\|^{2}-\lambda \operatorname{Tr}\left(W L W^{t}\right)\right),
$$

where $\operatorname{Tr}$ is the trace of a matrix and $L=D-A$ is the Laplacian matrix of $G$. Laplacian matrix is defined as $L=D-A$, such that $A$ is the weighted adjacency matrix of $G$ and $D$ is a diagonal matrix with $d_{i i}$ equal to the sum of the elements in the $i$-th row of $A$. The parameter $\lambda \geq 0$ (smoothness parameter) controls the influence of the regularization in the optimization process.

In a data mining context, the classical NMF formulation assumes a collection of $n$ items and $m$ features represented in a matrix $X \in R_{+}^{m \times n}$. However, an important class of clustering methods views the data points in a network model as a $n \times n$ similarity (or weighted adjacency) matrix $A$, wherein $n$ is the number of data points or vertices. Kuang, Ding and Park (2012) proposed a more general formulation for NMF-based clustering called Symmetric NMF (SymNMF), and the interpretability of NMF to the symmetric case is:

$$
\min _{H \geq 0}\left\|A-H^{t} H\right\|_{F}^{2}
$$

where $H$ is a non-negative matrix of size $n \times k, k$ is the number of clusters requested, and the largest entry $h_{i j}$ in column $h_{j}$ indicates that vertex $j$ belongs to cluster $i$.

Dias et al. (2017) used the previous definitions to propose an edge-ranking method that rewrites the edge weights based on an alternative measure of vertex similarity, computed from matrix $H$ yielded by either NMF, GNMF or SymNMF. The similarity between vertexes $j$ and $s$ is defined as the cosine of the angle between the corresponding columns in $H$ (i.e. between the probability vectors $h_{j}$ and $h_{s}$ ), multiplied by their norms, so:

$$
\operatorname{similariy}(j, s)=\sum_{1}^{k} h_{i j} h_{i s}=<h_{j}, h_{s}>=\left\|h_{j}\right\|\left\|h_{s}\right\| \cos \left(h_{j}, h_{s}\right)
$$


The computed similarity is assigned as the weight of the edge $(j, s)$. Vertex pairs with high edge-weights are matched using the GHEM matching strategy.

\section{B.5 Vertex-selection matching for bipartite networks}

Algorithm 8 describes the RGMb matching algorithm. In the algorithm, a vertex $u$ is picked randomly at each iteration, and as long as it has not yet been matched, one of its unmatched 2-hop neighbor $v$ with the maximal $S_{c n}$ (number of common neighbors) is chosen. The process iterates until no more vertices can be eliminated from the list. For unweighted edges, a random neighbor is selected for matching, otherwise, the heaviest adjacent edge is selected.

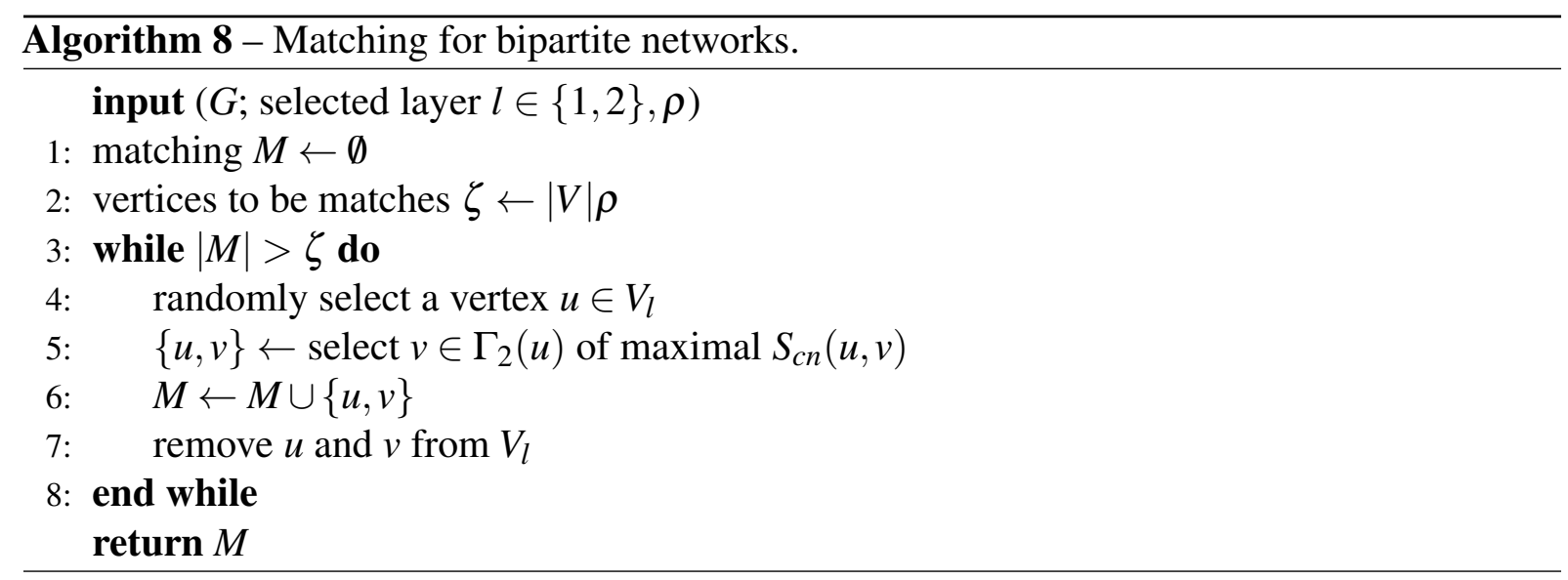

\section{B.6 Greedy matching based on modularity maximization}

In the Louvain algorithm, iteratively, each vertex $u$ is assigned to the community with a maximum positive gain (it may remain in its current community). The process continues until no further improvement can be achieved, i.e. when no individual move will improve modularity. The modularity gain $\Delta Q_{u \rightarrow C_{i}}$, of moving the vertex $u$ into the community $C_{i}$, is defined by:

$$
\Delta Q_{u \rightarrow C_{i}}=\left[\frac{C_{i}^{\text {in }}+\kappa_{u \rightarrow C_{i}}}{2 m}-\left(\frac{C_{i}^{\text {out }}+\kappa_{u}{ }^{2}}{2 m}\right)\right]-\left[\frac{C_{i}^{\text {in }}}{2 m}-\left(\frac{C_{i}^{\text {out }}}{2 m}\right)^{2}-\left(\frac{\kappa_{u}}{2 m}\right)^{2}\right]
$$

Algorithm 9 shows the basic structure of a local greedy matching based on modularity maximization. The algorithms have runtime complexity $O(m+n)$.

Schuetz and Caflisch (2008a), Schuetz and Caflisch (2008b) presented a Multi-Step Greedy matching algorithm, later improved by Noack and Rotta (2009), that prevents an extremely unbalanced growth of the clustering. These studies introduced a reduction factor parameter $\rho$ independent of network size, which can be set as a percentage of the number of the modularity-increasing cluster pairs. Schuetz and Caflisch (2008b) reported that an appropriate value of $\rho$ is $\sqrt{\kappa_{G}}$. The authors also introduced a merge prioritizer concept that assigns to each 


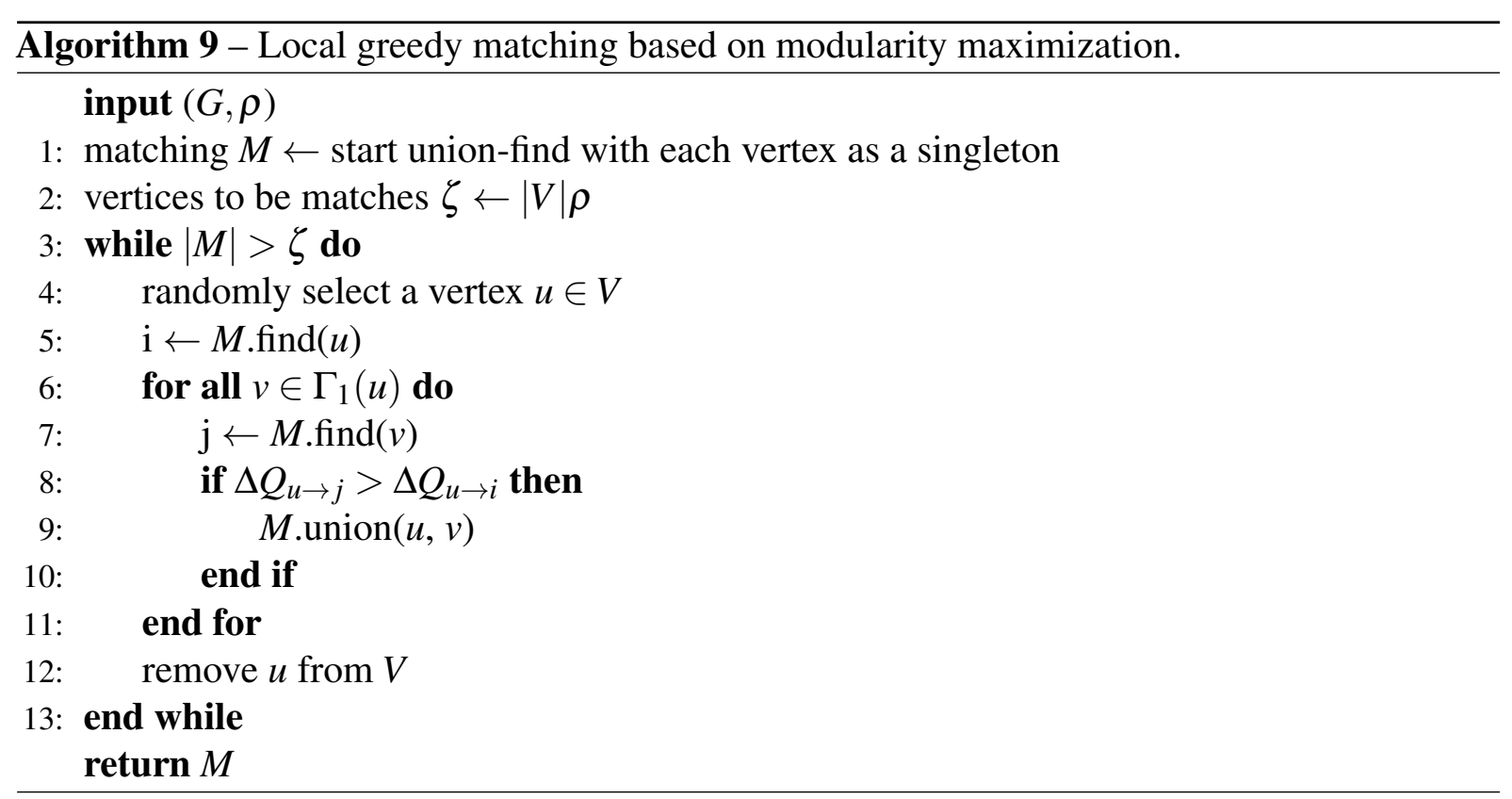

cluster pair $(C, D)$ a real number called merge priority. Noack and Rotta (2009) reported a list of merge prioritizers based on modularity gain, namely: Modularity Increase (MI), Weight Density (WD), Significance (SG), Danon et al (DN). and Wakita and Tsurumi (WT), defined respectively in Equations 2.12, 2.16, 2.14, 2.15, 2.13:

$$
\begin{gathered}
M I_{C_{i}, C_{j}}=\Delta Q_{C_{i}, C_{j}}=\frac{2 \kappa_{C_{i} \leftrightarrow C_{j}}}{\kappa_{G}}-\frac{2 C_{i}^{i n} C_{j}^{i n}}{\kappa_{G}^{2}} \\
W T_{C_{i}, C_{j}}=\min \left(\frac{\left|C_{i}\right|}{\left|C_{j}\right|}, \frac{\left|C_{j}\right|}{\left|C_{i}\right|}\right) \Delta Q_{C_{i}, C_{j}} \\
S G_{C_{i}, C_{j}}=\frac{\Delta Q_{C_{i}, C_{j}}}{\sqrt{C_{i}^{i n} C_{j}^{i n}}} \\
D N_{C_{i}, C_{j}}=\frac{\Delta Q_{C_{i}, C_{j}}}{\min \left(C_{i}^{i n} C_{j}^{i n}\right)} \\
W D_{C_{i}, C_{j}}=\frac{\Delta Q_{C_{i}, C_{j}}}{C_{i}^{i n} C_{j}^{i n}}
\end{gathered}
$$

Based on Schuetz and Caflisch (2008b) and Noack and Rotta (2009), Algorithm 10 shows the general structure of the matching strategy based on merge prioritizers. It iteratively visits the cluster pairs in descending order of merge priority, merging two clusters if there is a modularity gain.

Meyerhenke, Sanders and Schulz (2014) proposed a novel matching based on the label propagation algorithm (LPA, proposed by Raghavan, Albert and Kumara (2007), for community detection, and has a near-linear time: $O(n+m)$ in each iteration). In LPA, each vertex is initialized 


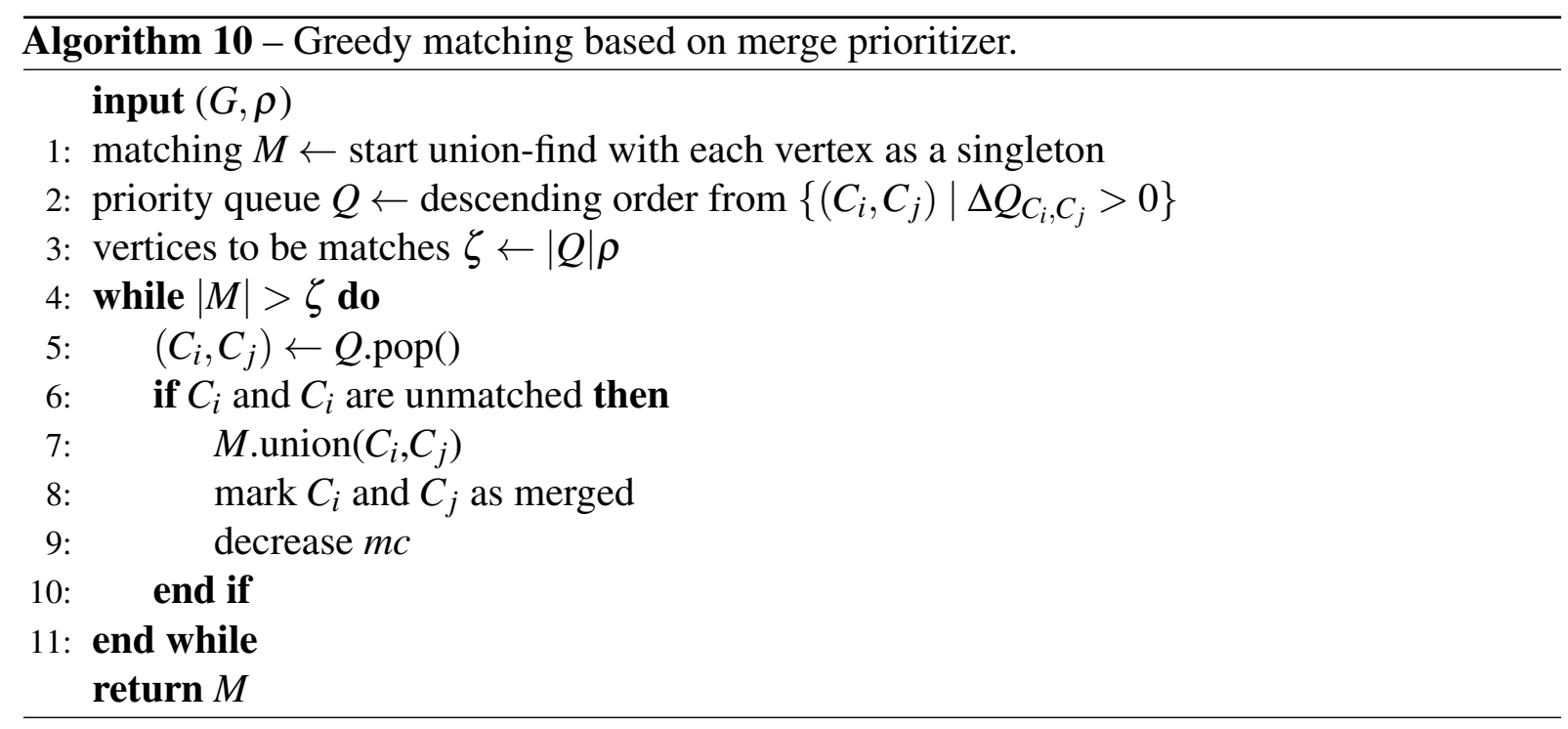

as a singleton. Then the vertices are visited in a random order. When a vertex $u$ is visited, it is moved to the cluster $C_{i}$ that its priority neighbor belongs to. The priority neighbor $v$ has maximum edge-weight over all valid edges incident to $u$, i.e. $\max \left(\left\{\omega(u, v) \mid u \in \Gamma_{1}(v)\right\}\right)$. Note that in the original formulation of LPA the visited vertex $u$ will belong to the cluster $C_{i}$ that occurs with the highest frequency among its neighbors. The process is repeated until the convergence or a number of iterations. Meyerhenke, Sanders and Schulz (2014) tunned the algorithm to stop if less then five percent of the vertices changed its clusters in an iteration. Vertices belonging to the same cluster are matched with each other. Algorithm 11 summarizes a general matching strategy based on the LPA.

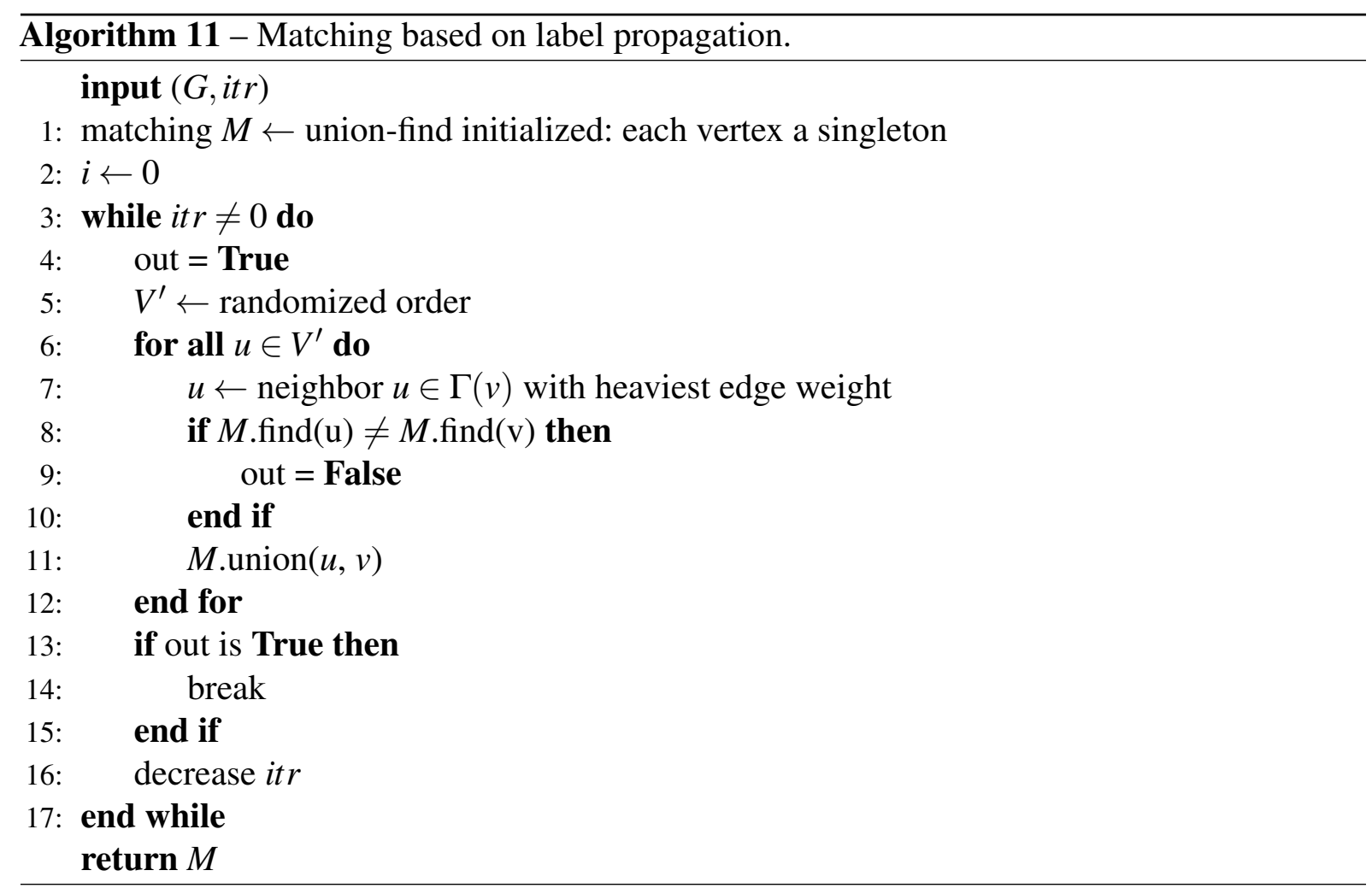




\section{B.7 Kernighan-Lin (KL) refinement algorithm}

Kernighan-Lin (KL) heuristic performs incremental operations by moving vertices between a bisection so as to reduce its edge-cut count. Let a balanced bisection be $C=\left\{C_{1}, C_{2}\right\}$, thus $\left|C_{1}\right|=\left|C_{2}\right|$. The method introduces two concepts: internal and external cost of a vertex $u$ in a partition $C_{i}$, defined by its internal $k_{u}^{\text {int }}$ and external degree $k_{u}^{\text {out }}$, respectively. Let be $D(u)$ the difference between the internal and external cost of $u$ in a partition $C_{i}$ :

$$
D(u)=k_{u}^{e x t}-k_{u}^{i n t}
$$

Let two vertices $u$ and $v$ belong to distinct partitions, i.e. $u \in C_{1}$ and $v \in C_{2}$. The gain $g$ resulting from the vertex exchange between partitions is:

$$
g(u, v)=D(u)+D(v)-2 w_{u, v}
$$

Algorithm 12 summarizes the refinement process based on KL. Let an initial solution, a bisection, be $S_{l}=\left\{C_{1}, C_{2}\right\}$ such that $C_{1}=\left\{u_{i} \mid 1 \leq i \leq n / 2\right\}$ and $C_{2}=\left\{v_{j} \mid 1 \leq i \leq n / 2\right\}$. The algorithm runs $q$ iterations, and in each finds a series of mutual exchange operations between vertices $u_{i} \in C_{1}$ and $v_{j} \in C_{2}$ that maximizes $g$.

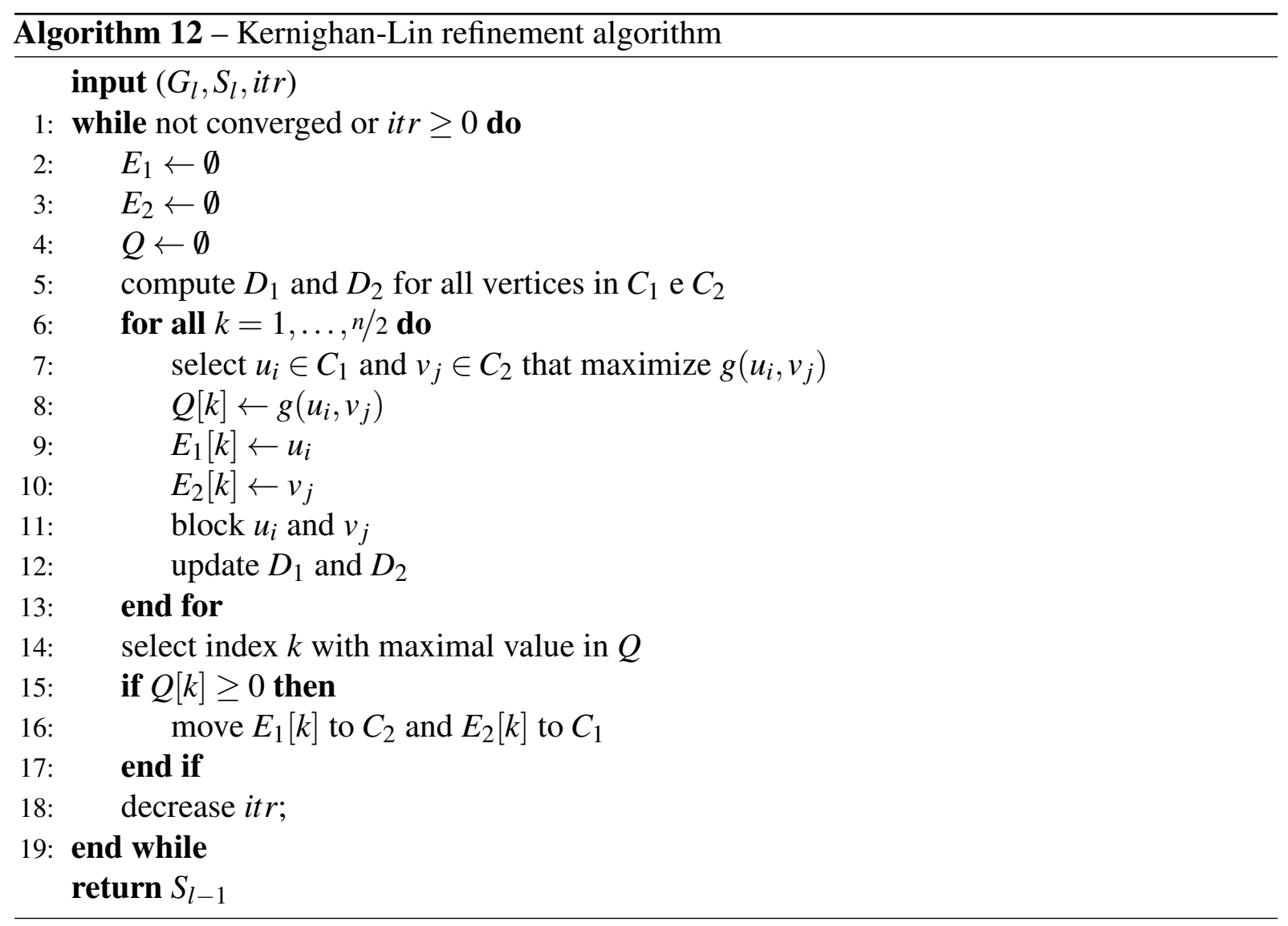


Initially, the ordered sets $D_{1}$ and $D_{2}$ are computed by:

$$
\begin{aligned}
& D_{1}=D\left(u_{1}\right) \geq D\left(u_{2}\right) \geq \ldots \geq D\left(u_{n / 2}\right) \\
& D_{2}=D\left(v_{1}\right) \geq D\left(v_{2}\right) \geq \ldots \geq D\left(v_{n / 2}\right)
\end{aligned}
$$

Vertices $u_{i}$ and $v_{j}$, selected in the iteration $q$, are blocked and will not be analyzed in the next iterations. The values of $D_{1}$ and $D_{2}$ must be recalculated using only the unlocked vertices, Equation 2.20, which implies in a new bisection $C^{\prime}=\left\{C_{1}-u_{i}, C_{2}-v_{j}\right\}$. The algorithm stops when there are no more unlocked vertices or in predetermined number of iterations.

$$
\begin{aligned}
& D_{1}^{\prime}(u)=D_{1}(u)+2 w_{u, x}-2 w_{u, x} \forall x \in C_{1}-u_{i} \\
& D_{2}^{\prime}(v)=D_{2}(v)+2 w_{v, x}-2 w_{v, x} \forall x \in C_{2}-v_{j}
\end{aligned}
$$



CHAPTER

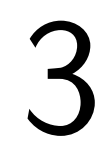

\section{A BENCHMARKING TOOL FOR THE GENERATION OF BIPARTITE NETWORK MODELS WITH OVERLAPPING COMMUNITIES}

Many real-world networks display hidden community structures with important potential implications in their dynamics. Many algorithms highly relevant to network analysis have been introduced to unveil community structures. Accurate assessment and comparison of alternative solutions are typically approached by benchmarking the target algorithm(s) on a set of diverse networks that exhibit a broad range of controlled features, ensuring the assessment contemplates multiple representative properties. Tools have been developed to synthesize bipartite networks, but none of the previous solutions address the issue of generating networks with overlapping community structures. This is the motivation for the BNOC tool introduced in this paper. It allows synthesizing bipartite networks that mimic a wide range of features from real-world networks, including overlapping community structures. Multiple parameters ensure flexibility in controlling the scale and topological properties of the networks and embedded communities. BNOC's applicability is illustrated assessing and comparing two popular overlapping community detection algorithms on bipartite networks, namely HLC and OSLOM. Results reveal interesting features of the algorithms in this scenario and confirm the relevant role played by a suitable benchmarking tool. Finally, to validate our approach, we present results comparing networks synthesized with BNOC with those obtained with an existing benchmarking tool and with already established sets of synthetic networks, in two different scenarios. 
Chapter 3. A benchmarking tool for the generation of bipartite network models with overlapping 86 communities

\subsection{Introduction}

The dynamics of many real-world systems is determined by the relations established between individual entities. This is the case, for example, of social networks, biological networks, information and technology networks (NEWMAN, 2010). Community structures in such complex networks are defined as a set of highly cohesive subsets of vertices and edges, also called components, partitions, or clusters. Such communities may be disjoint (FORTUNATO, 2010; JONNALAGADDA; KUPPUSAMY, 2016; VALEJO et al., 2018b), i.e., each vertex belongs to a single community, or overlapping (REES; GALLAGHER, 2012; VALEJO; VALVERDEREBAZA; LOPES, 2014c; VALEJO et al., 2014), when vertices may participate in multiple communities. The latter scenario is common in social networks, where individuals typically interact within multiple groups. Given a problem or a phenomenon described by a network model, vertices within a community typically share common characteristics or play similar roles.

Despite its relevance for characterizing a network's behavior, the community structure is unknown a priori. Yet, the analysis of network properties and dynamics demands a thorough understanding of its implicit topological organization. Community detection algorithms have been designed (FORTUNATO, 2010) to infer the actual organization of network vertices in a community structure. Their performance is typically measured in terms of the accuracy in identifying known representative and meaningful communities, combined with an analysis of computational complexity or execution times. Also relevant is how to accurately compare a novel algorithm with established ones. This is often accomplished by means of an empirical investigation conducted over a representative set of benchmark networks for which the community structure is known, so that results are amenable to validation and statistical analysis of the observed differences in performance. The benchmark networks should reflect the wide variety of features characterizing community structures, which may differ, e.g., in the number and size of communities, vertex degree distribution, presence of overlapping structures and also their extent and cohesiveness.

Benchmark networks representative of a diverse range of community organizations are necessary to disclose the limitations and advantages of the algorithms under investigation. However, obtaining ground-truth real-world networks exhibiting all the range of required features is hardly feasible, motivating the development of benchmarking tools to generate benchmark networks. The availability of such tools is paramount to support standardized, replicable and consistent empirical comparison of alternative algorithms (RABBANY et al., 2013). A wellknown contribution to the topic is the work by Lancichinetti, Fortunato and Radicchi (2008). Nonetheless, authors of a recent publication on the generation of hierarchical benchmark networks for community detection state that solutions contemplating specific scenarios are still lacking (YANG; PEROTTI; TESSONE, 2017).

Bipartite networks, also known as two-layer networks, comprise a particular category of relational models in which the set of vertices is split into two disjoint sub-sets (the layers) 
and edges only occur between vertices in different layers. Let $G=(V, E, \omega)$ be an undirected weighted network, where $V=\{1, \ldots, n\}$ denotes the set of vertices, $E \subseteq V \times V$ is the set of edges, and $\omega$ is the set of weights assigned to edges. $|V|$ and $|E|$ denote, respectively, the number of elements in the vertex and in the edge sets. A network $G=(V, E, \omega)$ is bipartite if $V=V_{0} \cup V_{1}$, such that $V_{0} \cap V_{1}=\emptyset$ and $E \subseteq V_{0} \times V_{1}$.

This is a highly frequent class of networks that result from modeling pairwise relationships between entities of different types, e.g. documents and terms (FALEIROS; ROSSI; LOPES, 2017), patient and gene expression (or clinical variables) (HWANG et al., 2008), individuals and songs (or books, or films) (GRUJIĆ, 2008), scientific papers and their authors (NEWMAN, 2001a; NEWMAN, 2001b). Despite their widespread occurrence as models of real-world problems, the offer of benchmarking tools to assess community detection algorithms on bipartite networks is rather limited. Existing benchmarks are either not accessible to researchers or lack proper documentation. Indeed, the strong interest in novel algorithms to detect overlapped communities in bipartite networks is not matched by an offer of established benchmarking tools (DU et al., 2008; LEHMANN; SCHWARTZ; HANSEN, 2008; LI; ZHANG; ZHANG, 2015; SOUAM; AITELHADJ; BABA-ALI, 2014; CUI; WANG, 2014; ZHANG; AHN, 2015).

In this paper we address this gap and introduce BNOC, a tool for synthesizing bipartite network models with varied features representative of properties from real networks. Multiple input parameters can be manipulated to create networks of varying sizes and with distinct community patterns in terms of number, size, balance, edge distribution intra- and inter-communities, degree of overlapping and cohesion, and degree of noise in the connection patterns.

An empirical analysis varying the parameter settings confirmed the tool enables synthesizing community structures representative of a wide range of real-world bipartite networks. Our results show that BNOC can generate networks with hundreds of thousands vertices and hundreds of millions edges in acceptable times whilst demanding reasonable computing resources. The tool has been employed to benchmark HLC and OSLOM, two well-known overlapping community detection algorithms applicable to bipartite networks. Empirical results confirm its usefulness in uncovering the strengths and limitations of both algorithms. Finally, BNOC has been compared with an existing benchmarking tool and with already established sets of synthetic networks to assess its reliability.

The remainder of the paper is organized as follows: Section 3.2 reviews related work on generating benchmarking models; Section 3.3 introduces the tool, its implementation and resources; Section 3.4 reports results from an empirical study that illustrates its potential to create representative bipartite networks to benchmark community detection algorithms; Section 3.5 presents the concluding remarks; finally, Appendix 3.A outlines the generalization of BNOC to address k-partite and heterogeneous networks. 
Chapter 3. A benchmarking tool for the generation of bipartite network models with overlapping

\subsection{Related Work}

Vast collections of real-world networks are available from repositories such as the Stanford Large Network Dataset Collection ${ }^{1}$, the Koblenz Network Collection ${ }^{2}$, NetWiki ${ }^{3}$, the UCI Network Data ${ }^{4}$, the Newman Network Data ${ }^{5}$ and Pajek ${ }^{6}$. However, the community organization of real networks is not known a priori, and accurate identification of outliers, overlapping vertices, community shapes and other potentially relevant features would demand extensive effort.

It is more feasible to rely on synthetic networks than to search for typical patterns in real-world problems, or take real networks as ground truth. A controlled variation of the relevant features of community structures embedded in a representative set of networks is an essential resource in establishing standardized and reusable procedures for algorithm assessment and comparison. Solutions exist to instantiate networks based on a particular underlying model, e.g. the scale-free network discussed by Barabasi and Bonabeau (2003), or the scale-free network with bipartite structure introduced by Birmelé (2009).

Papers that introduce novel community detection algorithms often report studies on synthetic networks, but such networks are seldom made available for reuse in future comparisons. It is possible to generate synthetic bipartite networks for benchmarking purposes with libraries such as Igraph ${ }^{7}$ and NetworkX ${ }^{8}$, e.g. fully connected, random and degree-based models, but they do not offer mechanisms to introduce arbitrary community structures.

There are benchmarks designed to assess community detection algorithms (MOUSSIADES; VAKALI, 2009; LARGERON et al., 2015), as well as tools for building realistic benchmarks of on-line social networks (BARRETT et al., 2009; PHAM; BONCZ; ERLING, 2013; ALI et al., 2014; PÉREZ-ROSÉS; SEBÉ, 2014; PASTA; ZAIDI, 2016; NETTLETON, 2016). Recent studies of large-scale social networks have resorted to simulated models (PHAM; BONCZ; ERLING, 2013; ARMSTRONG et al., 2013; BONCZ, 2013; CAPOTA et al., 2015), e.g. the GN (Girvan and Newman) model (GIRVAN; NEWMAN, 2002) has been widely employed in this context. A popular, accessible and documented benchmark, known as the LFR model Lancichinetti, Fortunato and Radicchi (2008), Lancichinetti and Fortunato (2009) generates networks following a power-law vertex degree distribution (CHAKRABARTI; ZHAN; FALOUTSOS, 2004; AKOGLU; FALOUTSOS, 2009). The LFR model has been recently extended to construct hierarchical networks for purposes of benchmarking community detection

\footnotetext{
$<$ https://snap.stanford.edu/data/>

$<\mathrm{http}: / /$ konect.uni-koblenz.de/>

$<$ http://netwiki.amath.unc.edu/SharedData/SharedData>

$<$ https://networkdata.ics.uci.edu/>

$<$ http://www-personal.umich.edu/ mejn/netdata/>

$<$ http://vlado.fmf.uni-lj.si/pub/networks/data/>

$<$ https://igraph.org $>$

$<$ https://networkx.github.io/>
} 
algorithms (YANG; PEROTTI; TESSONE, 2017). Alessandro and Vittorio (2018) have discussed synthetic benchmarks to compare community detection and link prediction methods.

To the best of our knowledge, no existing tool addresses the specific problem of creating arbitrary bipartite networks with overlapping community structure for benchmarking purposes. There are contributions in the literature that describe usages of synthetic bipartite networks, e.g., Larremore, Clauset and Jacobs (2014) relied on a random model with added noise to create two sets of synthetic bipartite networks with disjoint communities to assess a stochastic block model community detection algorithm. Similarly, Barber (2007) and Beckett (2016) generated weighted bipartite networks with disjoint communities and Chakrabarti, Zhan and Faloutsos (2004) synthesized directed and weighted bipartite networks. A model by Melamed (2014) relies on density and disjoint community settings to create four categories of synthetic bipartite networks.

Availability of general bipartite networks for benchmarking, however, remains limited. Previous models are not necessarily accessible for reuse in later studies, or the information disclosed may be insufficient to ensure correct replication of the target networks. In fact, previous contributions do not address the problem of synthesizing reproducible bipartite network models with varying topological properties. Scripts, as provided, e.g. by Larremore, Clauset and Jacobs (2014) and Melamed (2014), allow reproducing their models in similar experiments but were not conceived as documented general-purpose tools to synthesize a variety of reproducible bipartite networks. Moreover, these previous contributions did not address the generation of bipartite networks with overlapping communities.

This scenario motivated the development of a general-purpose benchmark generator of bipartite networks that ensures standardization and reproducibility in assessing community detection and other important algorithms.

\subsection{A Tool for Benchmark Generation}

In this section we introduce the BNOC benchmarking tool for synthesizing weighted bipartite networks with overlapping community structures. It can be employed to create networks with balanced or unbalanced overlapping communities, heterogeneous community sizes, intraand inter-community edge density with varying average degrees and clustering coefficients. Table 7 lists the parameters that can be manipulated to create small or large-scale bipartite networks with distinctive features.

BNOC relies on the negative binomial distribution, defined in Equation 3.1, a discrete probability distribution that represents the number of possible failures in a sequence of Bernoulli 
trials prior to reaching a target number of successes.

$$
B N(N ; n, s)=\left(\begin{array}{c}
N+n-1 \\
n-1
\end{array}\right) s^{n}(1-s)^{n}
$$

In the previous equation $N+n-1$ is the number of trials, $n-1$ is the number of successes, and $s$ is the probability of success. Therefore, the negative binomial distribution gives the probability of $n-1$ successes and $N$ failures in $N+n-1$ trials with a success in the $(N+n)^{t h}$ trial.

Table 7 - Description of the controlling parameters in BNOC.

\begin{tabular}{lrrrl} 
Parameter & Type & Domain & Default & Description \\
\hline -v, --vertices & $|v|=2$ & {$[1,|V|] \subseteq \mathbb{Z}$} & {$[10,20]$} & Number of vertices for each layer \\
-c, --communities & $|c|=2$ & {$[1,|V|] \subseteq \mathbb{Z}$} & {$[2,2]$} & Number of communities for each layer \\
-p0, --probability0 & $\left|p^{0}\right|=c[0]$ & $(0,|V|] \subseteq \mathbb{R}$ & {$[0.3,0.7]$} & Probabilities for vertices in layer 0 for each community \\
-p1, --probability1 & $\left|p^{1}\right|=c[1]$ & $(0,|V|] \subseteq \mathbb{R}$ & {$[0.3,0.7]$} & Probabilities for vertices in layer 1 for each community \\
-b, --balanced & boolean & $\{0,1\}$ & 0 & Boolean balancing flag that suppresses -p parameter \\
-x, --overlap & number & {$\left[0,\left|V_{0}\right|\right] \subseteq \mathbb{Z}$} & 1 & Number of overlapping vertices in $V_{0}$ \\
-y, --overlap & number & {$\left[0,\left|V_{1}\right|\right] \subseteq \mathbb{Z}$} & 1 & Number of overlapping vertices in $V_{1}$ \\
-z, --noverlap & number & {$[0,|c|] \subseteq \mathbb{Z}$} & 2 & Number of overlapping communities \\
-d, --dispersion & number & $\mathbb{R}$ & 0.1 & Dispersion of negative binomial distribution \\
-s, --success & number & $\mathbb{R}+$ & 1 & Probability of success \\
-n, --noise & number & $(0,1] \subseteq \mathbb{R}$ & 0.01 & Noise \\
-l, --normalize & boolean & $\{0,1\}$ & 0 & 0 -1 scale weight individually to unit norm \\
-u, --unweighted & boolean & $\{0,1\}$ & 0 & Unweighted bipartite networks
\end{tabular}

Source: Research data.

A negative binomial distribution can be generated as a gamma mixed Poisson distribution with $s=d / d+m u$ as gamma scale parameter, where $d$ is the target number of successful trials, or dispersion parameter; and $m u$ is a weighting factor that affects the shape of the distribution. Both parameters are strictly positive, i.e. $d$ and $m u \in \mathbb{R}_{+}$. If parameter $n$ in the probability distribution takes a non-integer value, the resulting probability is zero. Figure 31 illustrates the behavior of the negative binomial distribution for distinct choices of parameters $m u$ and $d$, where $k=10$ is the number of trials $(k=N+n-1)$.

Small dispersion values $(d \approx 0)$ result in strongly concentrated probability distributions approaching zero values, hence, likely to yield more failures. Otherwise, fewer failures occur as $d$ increases, as the concentration towards null values is not as pronounced. This behavior is useful in generating synthetic networks.

For this purpose, we associate the probability of a success with the probability of a connection (i.e., an edge) being established between two vertices, whereas a failure indicates no connection. Model construction departs from an initial bipartite network fully connected and unweighted. The negative binomial distribution is employed to compute the edge weights, 
wherein null probability values to compute the edge weights, wherein null probability values cause edges to be eliminated, and non-null probability values are assigned as weights of the corresponding preserved edges. Finally, it is possible to insert noise into the connection patterns initially established by the binomial distribution.

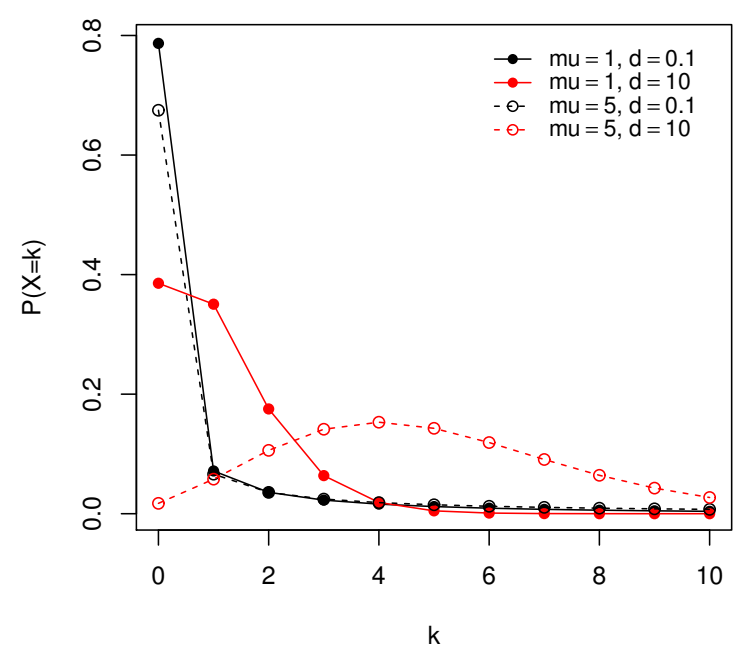

Figure 31 - Negative binomial distribution for distinct values of parameters $m u$ and $d$.

Source: Elaborated by the author.

Usually networks are created departing from an empty edge set, to which edges and corresponding weights are added one by one, iteratively, while considering some restriction, e.g., satisfying a desired degree distribution. The process typically requires several steps of edge rewiring, as part of connections considered may violate the imposed restriction (FORTUNATO, 2010). This increases the computational cost of the process. We adopt a more effective strategy, departing from a fully connected network with no weights and fitting a binomial distribution, which requires no iterative rewiring of undesired connections (though some rewiring is still required to establish noisy connections, see Step 5 below). The full procedure comprises the following steps:

\section{Step 1: Creating vertices and communities.}

Two initial input parameters define the desired number of vertices in each layer (array $v=\left[v_{1}, v_{2}\right]$, with $v_{1}=v_{2}$ or $\left.v_{1} \neq v_{2}\right)$ and the number of communities $(c)$. The relative size of each community is determined from a probability distribution $p^{0}=\left\{p_{1}^{0}, \ldots, p_{c}^{0}\right\} \forall p_{i}^{0} \in(0,1] \subseteq \mathbb{R}$ and $p^{1}=\left\{p_{1}^{1}, \ldots, p_{c}^{1}\right\} \forall p_{i}^{1} \in(0,1] \subseteq \mathbb{R}$ for layers 0 and 1 . Thus, a probability distribution $p_{j}^{i}$ determines the relative size of community $j$ in layer $i$. Higher probabilities will produce larger communities and, unless communities overlap, the sum of all community sizes must match the total number of vertices $|V|$. Alternatively, parameters $p^{0}$ and $p^{1}$ may be suppressed by a single parameter $b$ if the aim is to produce a balanced community structure, i.e., equal-sized communities. Furthermore, vertices must be assigned to communities: initially all vertices are created, then an iterative process assigns each one to a community randomly chosen with 
Chapter 3. A benchmarking tool for the generation of bipartite network models with overlapping

probability $p^{0}$ or $p^{1}$. Iteration stops once all vertices have been assigned.

\section{Step 2: Creating overlapping structures.}

Three optional input parameters $x, y$, and $z$ control community overlapping. Parameters $x$ and $y$ control, respectively, how many vertices from $V_{0}$ and $V_{1}$ will be assigned to multiple communities. Parameter $z$ defines the maximum number of communities each overlapping vertex will be assigned to. Overlapping vertices are selected randomly, defining a set $V^{\prime}$ with $\left|V^{\prime}\right|=x+y$. Similarly, overlapping communities are selected randomly, according to their respective probabilities $p_{j}^{i}$ as defined in the previous step. Each vertex $v \in V^{\prime}$ is assigned to up to $z$ randomly chosen communities, with probability $p^{0}$ or $p^{1}$. Higher values of $x, y$ will result in complex community structures more challenging to detect. These parameters are inspired by, and play a similar role, to those adopted in the widely adopted benchmarking solution by (FORTUNATO, 2010), which builds overlapping structures in one-layer networks.

\section{Step 3: Connecting vertices within communities.}

Vertices in the different layers that have been assigned to the same community are connected by an edge, so that all components become fully connected. Overlapping vertices are connected to all other vertices in their assigned overlapped communities, which produces components with overlapping regions.

\section{Step 4: Setting edge weights and network density.}

Each edge is assigned a weight obtained from the negative binomial distribution controlled by the dispersion parameter $d \in \mathbb{R}_{+}$and success probability parameter $s \in \mathbb{R}_{+}$, which can be set to control community density locally. Low values of $d$ and $s$ yield a distribution with many null values, hence causing more edges to be removed. Therefore, lower values of dispersion and success rate $(d \approx 0$ and $s \approx 0)$ yield sparser communities, whereas higher values $(d \approx 1$ and $s \approx 1)$ yield denser communities. Alternatively, parameter $l$ may be set to scale the weight vectors individually to the unit norm (vector length), and parameter $u$ may be set to create unweighted networks, i.e., the only role of the distribution is to control edge sparsity.

\section{Step 5: Adding inter-community edges and noise.}

After executing Step 4 each vertex has been connected to other vertices in its own community. An optional parameter $n$ enables inserting noise into the connection patterns at this point, so that the connections of randomly selected vertices will be rewired to vertices in other communities. Parameter $n \in(0,1] \subseteq \mathbb{R}$ induces a rewiring of a proportion of intracommunity edges, which are removed and replaced by randomly inserted inter-community edges, e.g. $n=0.5$ causes around $50 \%$ of the edges to be rewired. Low values of $n$ imply in inserting few inter-community edges, yielding networks with clear and easily separable communities. As $n$ increases, more inter-community edges are inserted, increasing overlap and causing community 
boundaries to become blurrier. In general terms, $n>0.5$ yields networks with poorly defined and sparse community structures depicting more inter-community than intra-community edges. Choices of $n \in[0.01,0.4]$ are often adequate to increase the difficulty of the community detection task without drastically harming the community structure.

It is worth mentioning that BNOC creates a community organization following the concept of communities as cohesive groups of "densely" connected vertices sparsely connected to vertices outside the group. The "well-behaved" and balanced initial structure created in Step 1 can be modified in later steps by introducing noise to connection patterns or increasing community overlapping. Other benchmarking tools, e.g. LFR, adopt a similar approach. Nonetheless, a precise formulation of what constitutes a community structure remains elusive and other tools may adopt different perspectives. Rosvall et al. (2017) describe a categorization of community detection algorithms and emphasize there is no general-purpose community detection algorithm, as applications and data types may differ in their perspective of what characterizes a community structure.

Figure 32 illustrates multiple networks obtained with a few parameter combinations (non-default parameter values are informed at the bottom of each plot, default values are as indicated in Table 7).

The network in Figure 32a exhibits a sparse topological structure, whereas Figure 32b illustrates a dense and unbalanced network. The network in Figure 32c is also dense and unbalanced, but it includes overlapping vertices. Finally, Figure 32d depicts a network with dense topology, extensive noise and overlapping vertices.

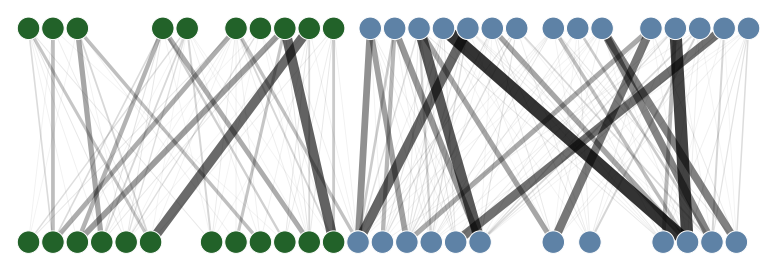

(a) $v=[25,15], c=[2,2], d=0.5, b$

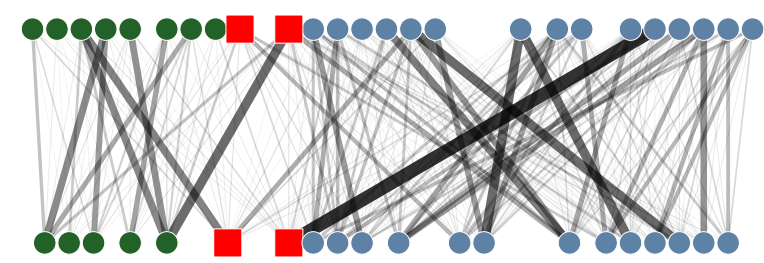

(c) $v=[20,25], c=[2,2], p 0=[0.4,0.6], p 0=p 1$ $x=2, y=2, z=2, d=0.6$
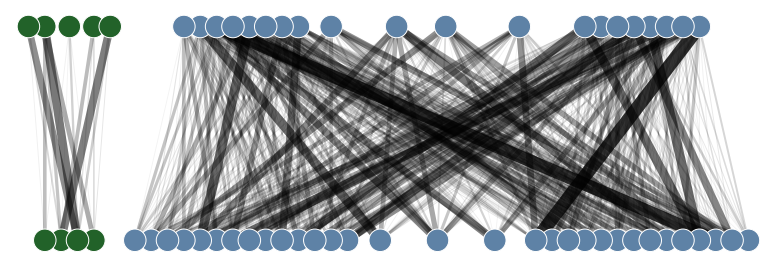

(b) $v=[35,25], c=[2,2], d=0.9, p 0=[0.2,0.8]$, $p 0=p 1$

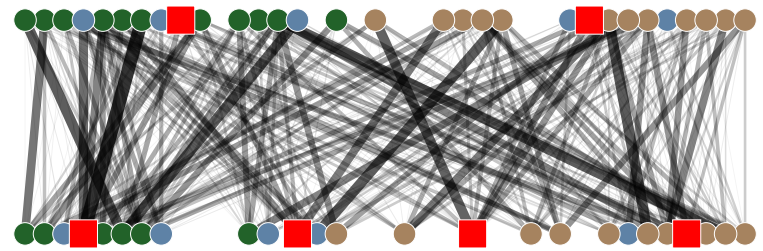

,(d) $v=[25,30], c=[2,2], d=0.8, x=4, y=2$, $z=2, n=0.2, b$

Figure 32 - Bipartite networks with distinct topological properties generated by BNOC. Line widths reflect the corresponding edge weights; red squares depict overlapping vertices and colored circles indicate non-overlapping vertices and their assigned community.

Source: Elaborated by the author. 
Chapter 3. A benchmarking tool for the generation of bipartite network models with overlapping

BNOC can be used to synthesize networks at varying levels of complexity that can be taken as good approximations of real-world models, which are often noisy and not necessarily "well-behaved". Such networks have blurred community boundaries and complex structures that may hinder algorithm performance and affect the community properties controlled in the aforementioned Steps 3 to 5. Their adjacency matrices, illustrated in Figure 33, show how a network's topological properties vary as different parameter settings are employed when executing Steps 3, 4 and 5.

Figure 33 illustrates four synthetic networks constructed executing Steps 3, 4 and 5 with different parameterizations. In Figure 33a, a network is initially created with a dense, balanced and non-overlapping community structure (Step 3); then, edge weights are created and some edges are removed (Step 4); finally, noise is added to the connection patterns (Step 5). In Figure $33 \mathrm{~b}$, the initial network with a balanced and overlapping community structure becomes sparser after executing step 4 and adding some noise (step 5). In Figure 33c an initial network with a dense, balanced, and overlapping community structure becomes sparser with extensive noise. Finally, in Figure 33d a network with an unbalanced overlapping community structure is modified to become highly sparse with extensive noise.
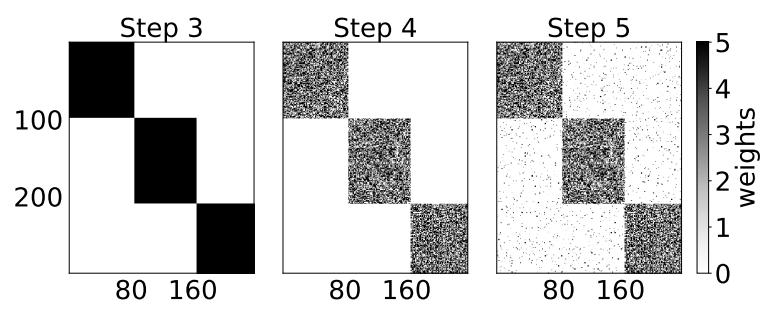

(a) $v=[300,240], c=[3,3], d=0.9, n=0.03$
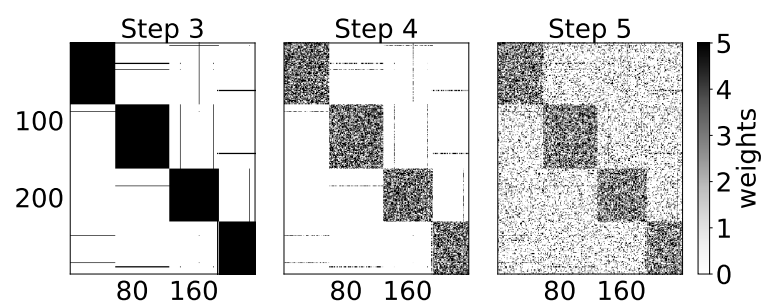

(c) $v=[300,240], c=[4,4], d=0.9, x=10, y=10, z=3$, $\mathrm{n}=0.2$
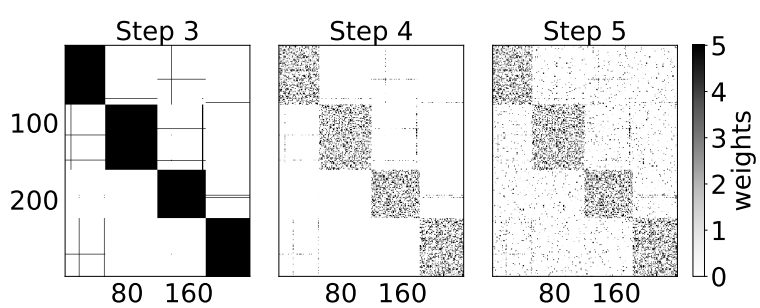

(b) $v=[300,240], c=[4,4], d=0.1, x=10, y=5, z=2$, $\mathrm{n}=0.03$
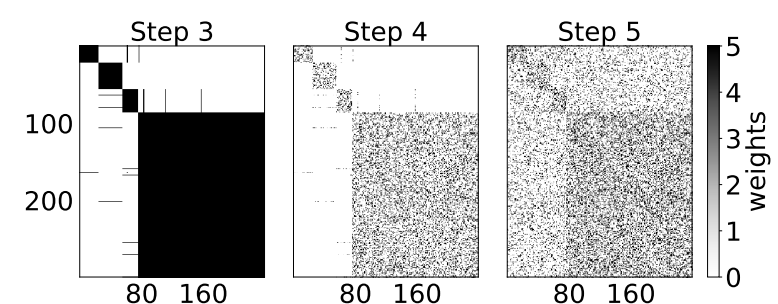

(d) $v=[300,240], \quad p 0=[0.1,0.1,0.1,0.7], \quad p 0=p 1$, $\mathrm{c}=[4,4], \mathrm{d}=0.1, \mathrm{x}=10, \mathrm{y}=5, \mathrm{z}=3, \mathrm{n}=0.2$

Figure 33 - Views of the adjacency matrices depicting how the topological properties of an initial network created in Step 3 are modified as Steps 4 and 5 are executed. Four scenarios are illustrated corresponding to alternative parameter settings: (a) an initial dense, balanced and non-overlapping community structure is modified by adding weights (causing some edges to be removed) and inserting limited noise; (b) a sparse, balanced, and overlapping community structure is modified with added weights and limited noise; (c) a dense, balanced, and overlapping community structure is modified with added weights and extensive noise; (d) a highly sparse, unbalanced, and overlapping community structure is modified with added weights and extensive noise. 
A few topological features are indirectly controlled by a combination of certain parameter choices. For instance, network average degrees and local clustering coefficients depend on parameters $c$ (number of communities), $p^{0}$ and $p^{1}$ (the probability distributions which define the size of each layer), $d$ (dispersion) and $s$ (probability of success). Denser bipartite networks resulting from higher dispersion values naturally yield higher average degrees and clustering coefficients.

Figure 34 illustrates the behavior of the average degree (LATAPY; MAGNIEN; VECCHIO, 2008) for networks of distinct sizes, as a function of parameter $d$ assuming $s=1$ fixed and a balanced layer structure ( $p^{0}$ and $p^{1}$ suppressed by setting parameter $b$ ). Lower dispersion values $(d \approx 0)$ yield lower average degrees (between 3 and 10), whereas higher dispersion values $(d \approx 1)$ yield high degree networks.
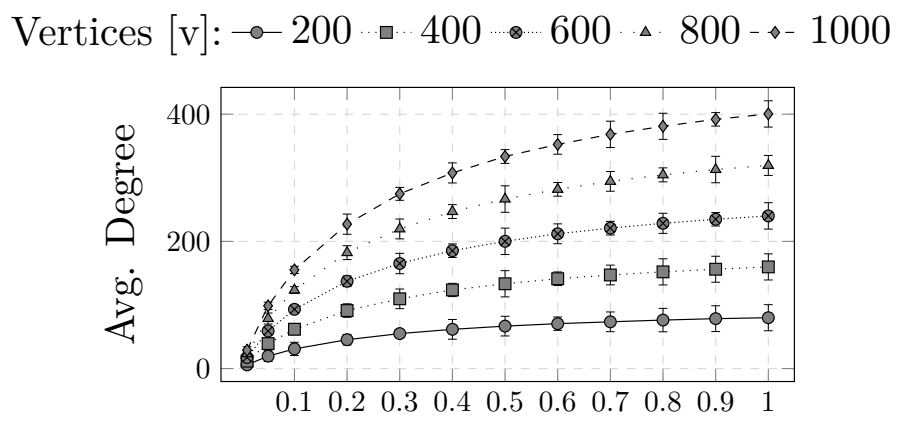

Dispersion $[\mathrm{d}]$

Figure 34 - Behavior of a network's average degree as a function of the dispersion parameter $d$.

Source: Elaborated by the author.

Figure 35 shows the behavior of the average clustering coefficient (LATAPY; MAGNIEN; VECCHIO, 2008) on four networks of distinct sizes, again as a function of dispersion parameter $d$. Low values result in networks with lower average clustering coefficients (between 0.1 and 0.2 ), whereas high values yield high clustering coefficients.

Appropriate parameterization of BNOC's underlying model enables simulating a wide range of behaviors representative of real-world networks. Figure 36a (top) shows the distribution of degree (left) and clustering coefficient (right) of a synthetic bipartite network with a few high-degree vertices and many low-degree. Figure $36 \mathrm{~b}$ (bottom) illustrates the same information for a network with no community structure, obtained setting parameter $c=[1,1]$, which leads to a topological structure close to a random model. According to Newman (2001a), in social networks vertex clustering coefficients and degree are typically inversely proportional.

Sparsity can be controlled by varying the edge distributions intra- and inter-community. In general, document-term networks (FALEIROS; ROSSI; LOPES, 2017) are sparse, whereas biological patient-gene expression (or patient-clinical) networks are characterized by dense structures (HWANG et al., 2008). The ability to independently control the size of each network layer allows modeling a topological property of many real networks, e.g. in biological patient- 


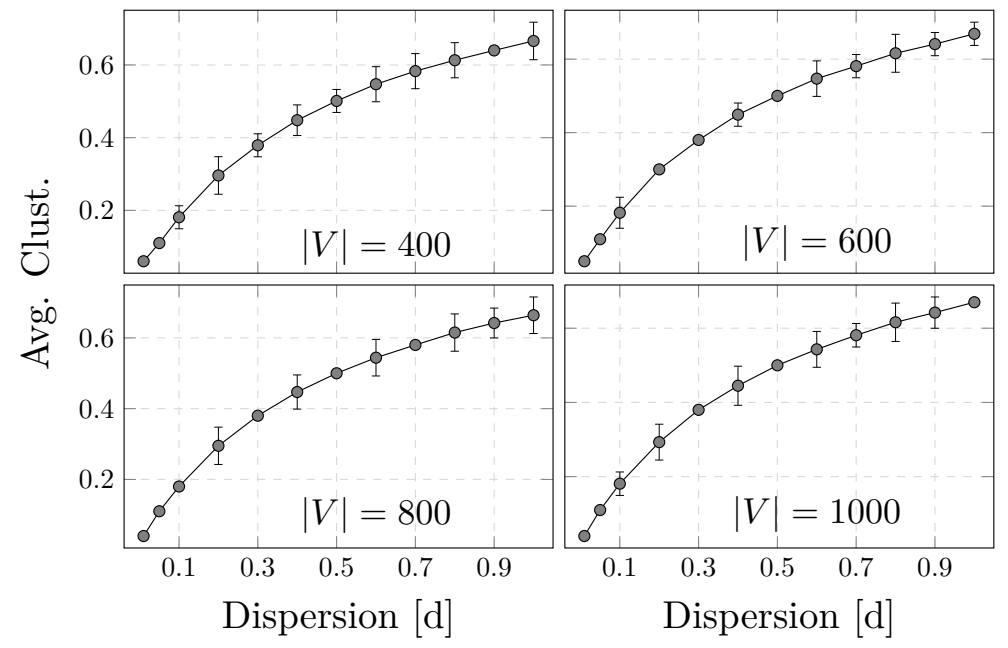

Figure 35 - Behavior of a network's average clustering coefficient as a function of dispersion parameter $d$, considering four network sizes (shown in separate graphs to avoid line overlapping).

Source: Elaborated by the author.

gene expression networks there are many more genes than patients. Likewise, document-term networks typically have many more terms than documents. Again, in order to reflect properties of real networks, community structure and degree of vertex overlapping are also controlled separately for each layer. The extent of noise is controlled independently of the overlapping structure, since it is important to observe if a community detection algorithm can distinguish between vertex overlapping, i.e., vertices that belong to multiple communities, and noise.
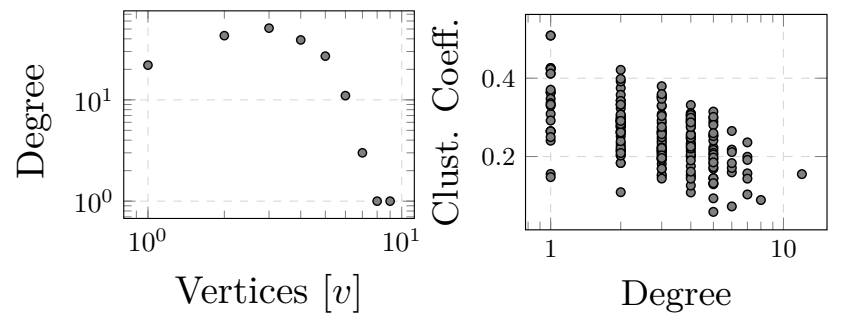

(a) $v=[100,100], d=0.1, c=[10,10], n=0.1, b$

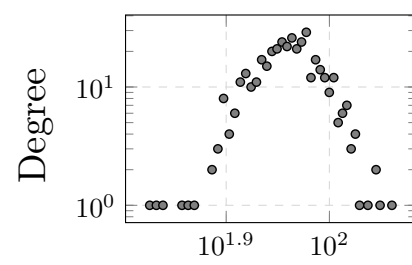

Vertices $[v]$

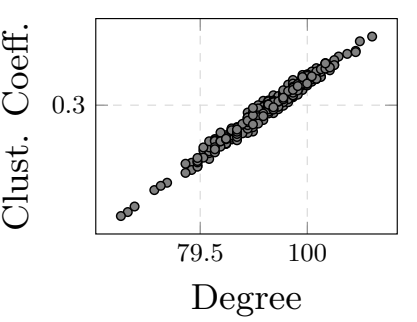

(b) $v=[200,200], d=0.2, c=[1,1], b$

Figure 36 - Distribution of vertex degree and local clustering coefficient of two distinct bipartite networks obtained with BNOC.

Source: Elaborated by the author.

Overlapping vertices are simultaneously assigned to up to $z$ communities randomly 
chosen considering the individual community probabilities $p_{j}^{i}$, as described in Step 2. Therefore, there will be vertices simultaneously assigned to exactly $z$ communities and others assigned to less than $z$ communities. Parameter $z$ allows creating overlapping structures of varying complexity: high values, i.e., $z \approx c$, yield to strong, explicit and easily detectable community overlapping, especially for high values of $c$, since the overlapping vertices will belong to many communities simultaneously. In contrast, low values, i.e., $z \approx 2$, yield to more subtle overlapping, since the overlapping vertices will simultaneously belong to just a few communities. Figure 37 illustrates the effect of varying parameter $z$ in a synthetic bipartite network initially with five communities in both layers. The red vertices are the overlapping ones, the remaining colors identify the vertices assigned to each of the distinct communities. Figure 37a exhibits the community structure of the network obtained with parameter settings $c=[5,5], z=5, x=y=50$; Figure 37b shows the community structure obtained with $c=[5,5], z=3$ and $x=y=80$; and Figure 37c results from setting $c=[10,10], z=2$ and $x=y=150$.

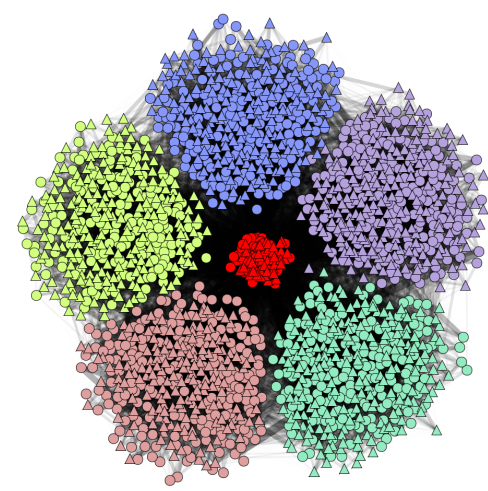

(a)

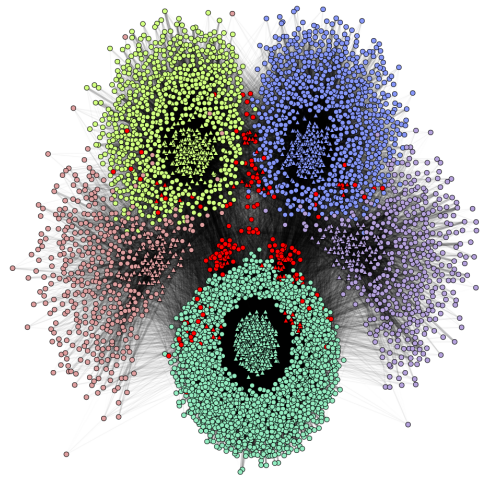

(b)

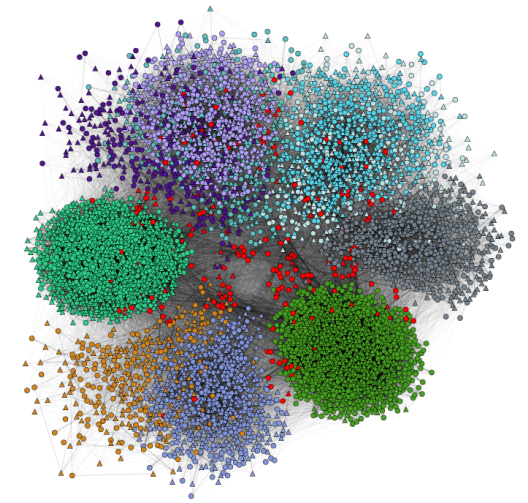

(c)

Figure 37 - Bipartite networks generated with BNOC illustrating the effect of varying parameter $z$, which controls the degree of community overlapping. Red markers depict overlapping vertices, whereas other colors indicate the assigned communities of the non-overlapping vertices. (a) a network built with five communities $(c=[5,5])$ and $z=5$ (strong overlapping); (b) a network built with $c=[5,5], z=3$, and $x=y=80$; (c) a network built with $c=[10,10], z=2$, and $x=y=150$.

Source: Elaborated by the author.

Specific parameters allow controlling the range and scale of edge weights to simulate the behavior of many real weighted or unweighted networks. Possible examples are user-movie networks such as IMDb (https://www.imdb.com/), in which registered users assign to films grades from 1 to 10; user and web-page networks, in which users may access a web-page (as Facebook) hundreds of times and others just a few times (as an on-line banking application) (both weighted networks); document-term networks, in which edges indicate the frequency of a term in a document (weights normalized to scale $0-1$, obtained setting parameter $l$ ); or unweighted location-based on-line social networks with geographic check-ins (obtained setting parameter $u$ ).

Figure 38 illustrates a BNOC-generated bipartite network that presents some of the afore- 
Chapter 3. A benchmarking tool for the generation of bipartite network models with overlapping

mentioned distinct behaviors at each layer, such as sparsity, weight scale, size and community structure derived with different probability distributions.

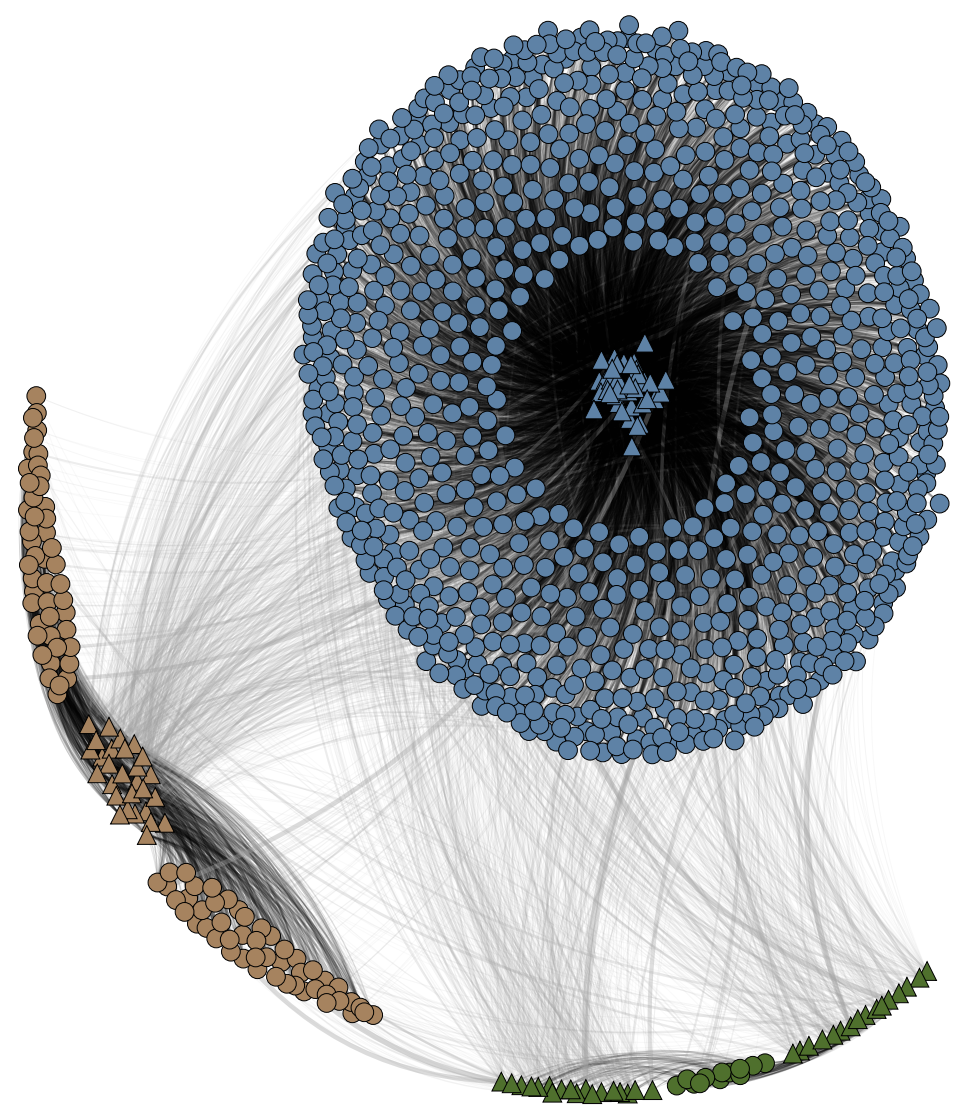

Figure 38 - Bipartite network generated with BNOC that illustrates properties of a hypothetical documentterm network with 1,000 terms (shown as circles) and 200 documents (shown as triangles) and three unbalanced communities. It has a sparse edge distribution, with normalized 0-1 weights. Globally the network has many more terms than documents, but this is not necessarily true of all communities: both the larger community in blue and the smaller community in brown have more terms than documents, unlike the smallest community, in green.

Source: Elaborated by the author.

It might represent, for instance, a hypothetical corpus of political opinions about a specific topic expressed in three types of media documents such as political discourses, tweets and journalistic news, modeled as a bipartite document-term network (FALEIROS; ROSSI; LOPES, 2017; AKOGLU, 2014). Globally, the term layer is considerably larger than the document layer, however, this global property does not necessarily hold locally in all communities. Let us say, it is possible that a community formed by the tweets might have more documents than terms (e.g., the green community in Figure 38), unlike the communities by the political discourses or media news (the brown and blue communities), or yet one of the communities might be balanced in the number of documents and terms.

Other complex community organizations could be considered in such a network, e.g., communities might model topics defined by multiple words, pronominal choices (or textual context), political parties or others. In each scenario, an investigation on how to parameterize 
the model to obtain the desired behavior would be required. While some features are directly controlled by individual parameter settings, others are determined by random or indirect choices.

Parameters $d$ and $s$ can be chosen in combination to generate sparse $(|E| \approx|V|)$ or dense networks $(|E| \gg|V|)$. Figure 39 shows curves of the number of edges (sparsity) as a function of the dispersion parameter $d$, for fixed values of the probability of success parameter $s$.

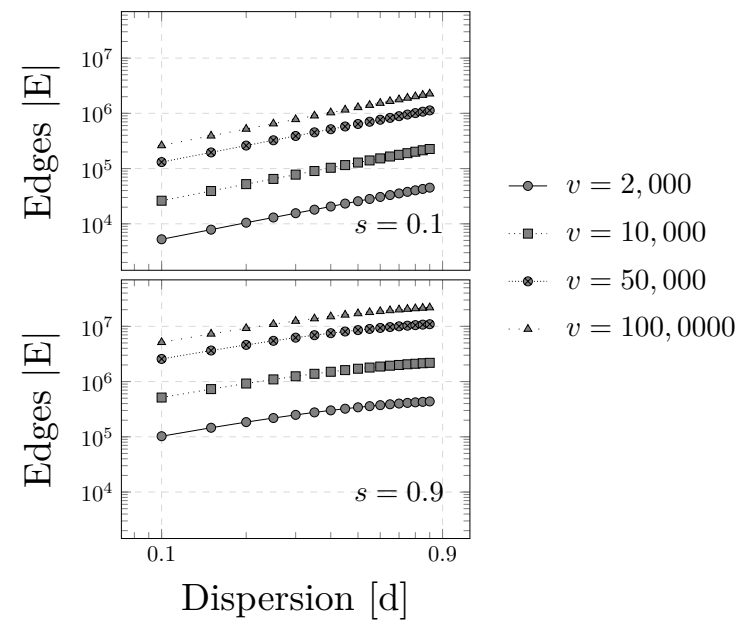

Figure 39 - Number of edges (sparsity) as a function of the dispersion parameter $d$.

Source: Elaborated by the author.

Considering vertex sets of sizes 2,000 and 100,000, respectively, picking e.g., $d=0.1$ and $s=0.1$, yielded sparse networks with 5,000 and 200,000 edges, respectively; on the other hand, picking $d=0.9$ and $s=0.9$ generated dense networks with 400,000 and 25,000,000 edges, respectively.

We assessed the scalability of BNOC to generate large networks. Networks were created with parameter $v$ varying in the range $[2,000 ; 100,000]$ at increments of 2,000 , setting the number of communities $c=0.01 v$, dispersion $d=0.1$, and probability of success $s=0.9$. The curves depicting the times to create the networks as a function of the number of vertices are shown in Figure 40.

On sparse networks ( $d=0.1$ and $s=0.1$ ), BNOC spent, in average, 0.2 and 19 seconds to build networks with $v=2,000$ and $v=100,000$ vertices, respectively. On dense networks ( $d=0.9$ and $s=0.9$ ) it spent, in average, 0.3 and 20 seconds to build networks with $v=2,000$ and $v=100,000$ vertices, respectively. Therefore, execution times increase with the number of vertices, but sparsity does not impact the tool's scalability.

As opposite, the number of communities directly impacts scalability. Since edges are created to connect vertices within the same community, a small number of large communities implies more edges, and many communities implies less edges. Networks were generated with $v=2,000,10,000,50,000$ and 100,000 vertices setting the number of communities $c$ within the range $[2,160]$ at increments of 2 , and $d=s=0.5$. Resulting execution times are shown 


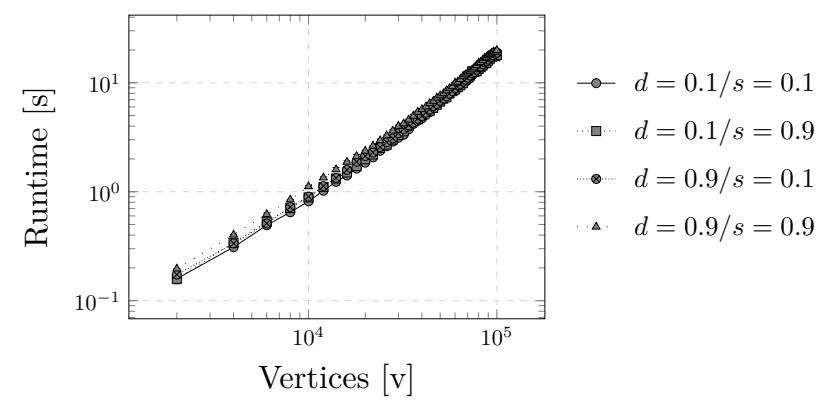

Figure 40 - Runtime as a function of the number of vertices $v$.

Source: Elaborated by the author.

in Figure 41. For large-scale networks, with 100,000 vertices, BNOC spent, in average, 643 seconds (or 10 minutes) to build networks with a single community and 14 seconds, in average, to build networks with 160 communities.

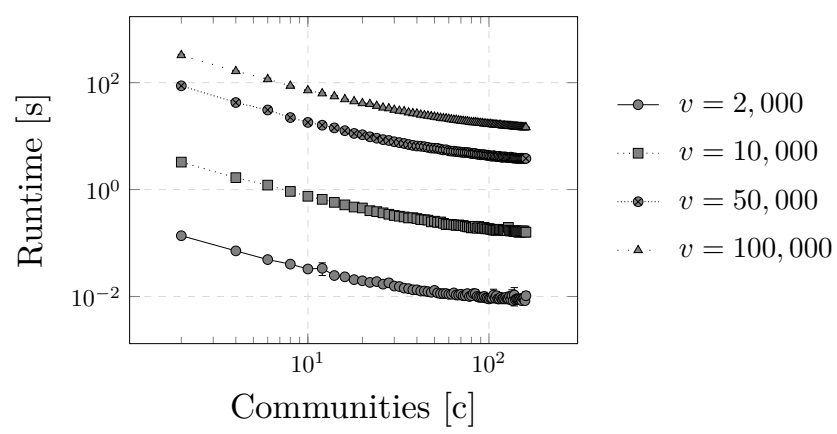

Figure $41-$ Runtime as a function of the number of communities $c$.

Source: Elaborated by the author.

We also compared BNOC with the well-known LFR benchmarking tool (LANCICHINETTI; FORTUNATO; RADICCHI, 2008; LANCICHINETTI; FORTUNATO, 2009) regarding execution times and scalability. We created networks with parameter $v$ varying in the range $[2,000 ; 100,000]$ at increments of 2,000 , setting the number of communities $c=0.01 v$ in both tools. Simulating a worst case scenario required generating dense networks, therefore, in BNOC we set dispersion $d=0.9$ and probability of success $s=0.9$; in LFR we set a high average degree $k=40$. The curves depicting the times to create the networks as a function of network size are shown in Figure 42.

While it took, in average, 0.3 and 20 seconds for BNOC to build dense networks with 2,000 and 100,000 vertices, respectively, it took 4.4 and 396 seconds (or 6.6 minutes) for LFR to build the equivalent networks. The tools are written in different programming languages (LFR in $C$ and BNOC in Python) and have different aims (LFR synthesizes simple networks, BNOC synthesizes bipartite networks) but this analysis confirms that BNOC is fast and scalable.

This discussion was aimed at describing and justifying the design choices for BNOC and illustrate its potential for generating diverse large-scale bipartite networks with tens or even 


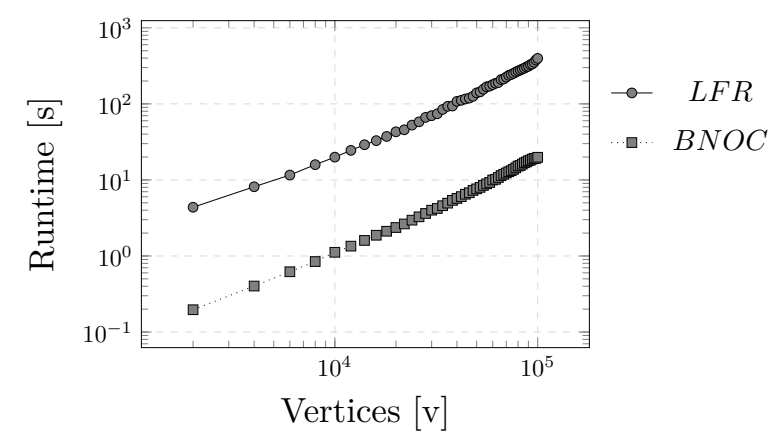

Figure 42 - Runtimes for obtaining synthetic networks of varying sizes in the LFR and BNOC benchmarking tools.

Source: Elaborated by the author.

hundreds of thousands vertices and hundreds of millions edges in a timely manner.

The time complexity is determined by the cost of generating the binomial distribution and other processes linear in $|V|$. In general, computing a probability of success is constant, therefore, drawing $v$ samples from a negative binomial distribution is $O(|V|)$. However, it depends on the implementation of the exponential and binomial coefficient functions. Our implementation relies on the numpy library ${ }^{9}$, in which the corresponding functions are linear time. The space complexity of the current implementation is $O\left(|V|^{2}\right)$ as the initial network is a complete network with $|V|$ nodes (CORMEN et al., 2009). Nonetheless, this cost could be reduced employing data structures for representing sparse matrices.

BNOC has been implemented using modular operations, so it can be easily generalized to other scenarios. In Appendix 3.A we outline some generic principles underlying its generalization to creating $k$-partite and heterogeneous networks, an extension that is already operational in the current version. Other scenarios may be not as straightforward, e.g., generalizing to creating directed networks requires special attention to certain connection properties.

\subsection{Empirical Evaluation}

We conducted two experimental studies. The first study investigated whether BNOC can support a standardized, replicable and consistent empirical comparison of the performance (regarding accuracy and runtime behavior) of different algorithms, and also how it can help unveiling the strengths and limitations of community detection algorithms. The second study was conducted for validation purposes, comparing the networks synthesized with BNOC with already established sets of synthetic networks, in two different scenarios.

All experiments (including those reported in Section 3.3) were conducted in a Linux machine with an 8-core $3.7 \mathrm{GHz}$ CPU and $64 \mathrm{~GB}$ main memory. Whenever applicable we report average values obtained from 30 executions for each algorithm.

9 http://www.numpy.org/ 
Chapter 3. A benchmarking tool for the generation of bipartite network models with overlapping 102

communities

\subsubsection{Support for consistent algorithm evaluation}

We used BNOC to create benchmark networks to assess the performance of two popular algorithms for overlapping community detection in bipartite networks, namely Hierarchical Link Clustering (HLC) (AHN; BAGROW; LEHMANN, 2010) and Order Statistics Local Optimization Method (OSLOM) (LANCICHINETTI et al., 2011). HLC focuses on grouping edges rather than vertices to reveal hierarchical overlapped organizations, whereas OSLOM is based on local optimization of a fitness function that expresses the statistical significance of clustering. Both algorithms are applicable to several network domains, however, their performance in bipartite models has not yet been assessed empirically. HLC can be directly applied to bipartite networks, whereas OSLOM can be applied to a one-mode projection of the bipartite network, which we did following the guidelines presented by (MELAMED, 2014). Besides comparing the algorithms' capabilities in bipartite networks, it is our goal to verify whether BNOC can create representative benchmarking networks capable of revealing their relevant characteristics, strengths and limitations.

The following three measures of algorithm performance were considered: normalized mutual information (NMI), accuracy (Acc) and runtime (seconds). Acc measures an algorithm's ability to identify both overlapping (sensitivity) and non-overlapping (specificity) vertices. It is defined as $(T P+T N) /(T P+F P+T N+F N)$, where TP is the number of actual overlapping vertices correctly classified, TN is the number of actual non-overlapping vertices correctly classified, FN is the number of actual overlapping vertices mistakenly classified as non-overlapping, and FP is the number of actual non-overlapping vertices mistakenly classified as overlapping. The NMI index relies on information theory to quantify the quality of the communities inferred; we employ the formulation extended by (MCDAID; GREENE; HURLEY, 2011) to handle overlapping communities.

NMI considers non-overlapping and overlapping structures, therefore, the number of vertices in each layer can exert direct influence on evaluation results. A set of 270 synthetic weighted bipartite networks was generated with the following parameter settings: $|V|$ varied within the range $[500,1000]$ at increments of 100 , so that $v=\left[v_{1}, v_{2}\right],|V|=v_{1}+v_{2}$ and $v_{1}=$ $0.8 v_{2}$, which creates unequally sized layers. The remaining parameters were set to $n$ within the range [0.1,0.3] at increments of $0.025, d$ varied within the range [0.5,0.9] at increments of $0.1, x=y=0.05|V|, z=0.01|V|$ and $c=0.02|V|$. Because HLC has high time and space complexities, experiments were limited to small networks.

Figure 43 depicts the NMI values for HLC and OSLOM, respectively, as a function of the amount of noise (parameter $n$ ) in networks of different sizes (parameter $v$ ).

The algorithms exhibit distinct behaviors, i.e., NMI values for HLC decrease quickly as noise levels increase (higher values of parameter $n$ ), whereas with OSLOM they decrease slowly. Indeed, increasing noise levels yields more inter-community edges, consequently, boundaries 

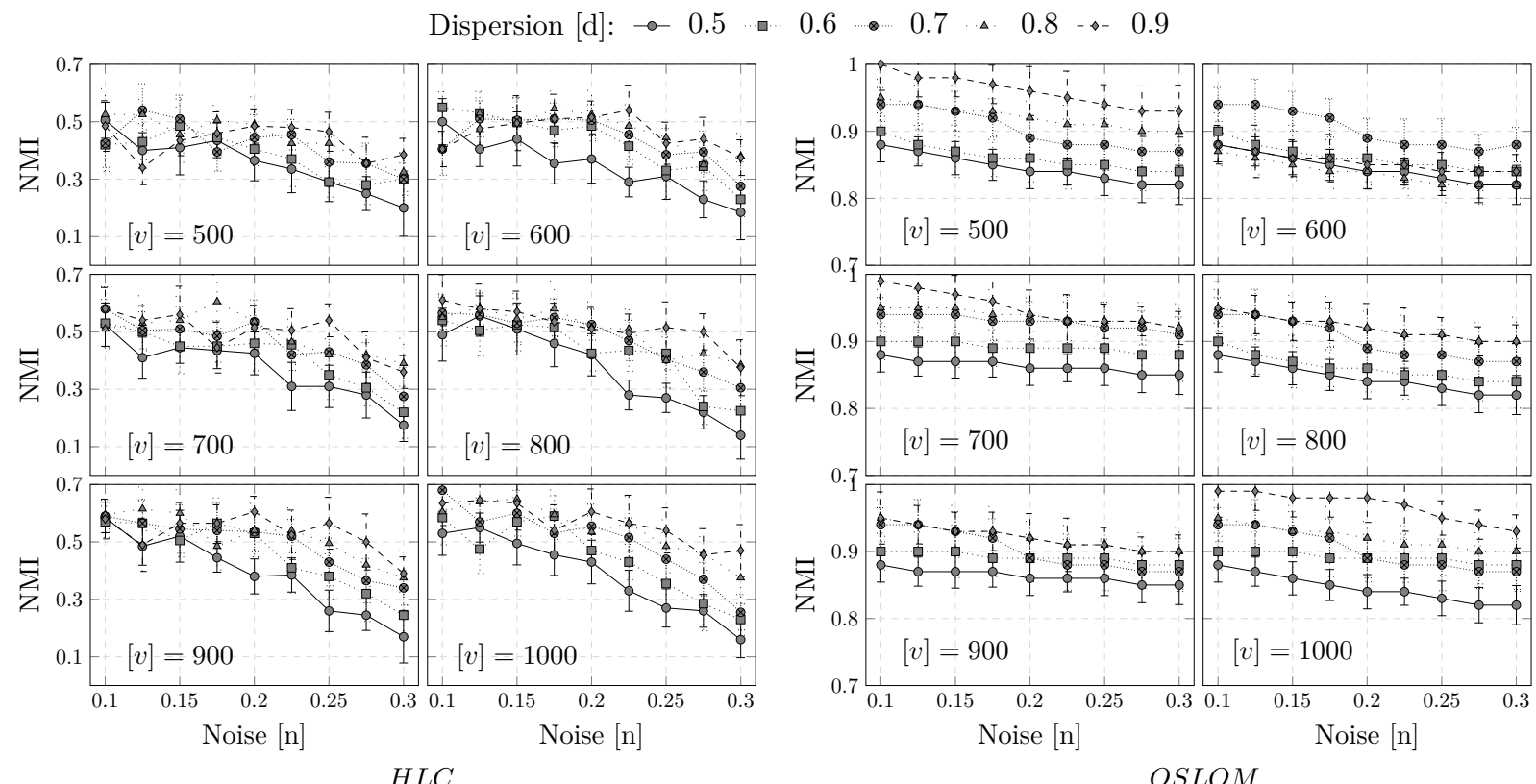

Figure 43 - NMI results for HLC and OSLOM as a function of noise obtained on 270 synthetic weighted bipartite networks of six different sizes $|V|$.

Source: Elaborated by the author.

between communities become more blurred and harder to identify. Whereas HLC's performance is evidently highly sensitive to noise levels, OSLOM results are more stable, suggesting its performance is not as strongly affected.

In both cases, higher dispersion values (parameter $d$, which controls network density) yielded higher values of NMI, as densely connected communities are more cohesive and easily separable. On the other hand, lower dispersion leads to sparser communities with blurred boundaries. In general, both HLC and OSLOM achieve good results regarding the quality of the communities identified, however, their performance is directly affected by the choice of $d$. In particular, quality degrades when more extensive noise levels are applied to sparser networks (low dispersion values).

The accuracy measure (Acc) quantifies the quality of the overlapping communities inferred by the algorithms, i.e. Acc depends on the number of overlapping vertices or communities, rather than layer size. Therefore, BNOC was employed to create 532 synthetic weighted bipartite networks of sizes $|V|=600$ and $|V|=900$, such that $v=\left[v_{1}, v_{2}\right],|V|=v_{1}+v_{2}$ and $v_{1}=v_{2}$, i.e., equal-sized layers. Parameters $x=y$ were varied within the range $[2,20]$ at increments of 1 , parameter $z$ varied within the range $[2,15]$ at increments of 1 , and remaining parameters were set to $n=0.1$ and $c=[15,15]$. Figure 44 shows the Acc values for HLC and OSLOM, respectively, as a function of the numbers of overlapping vertices (parameters $x$ and $y$ ) in Figure 44a), and overlapping communities (parameter $z$ ) in Figure 44b).

Figure 44a (left) shows the Acc values for HLC decrease slowly as the number of overlapping vertices increases. Furthermore, the algorithm exhibits a stable behavior when 


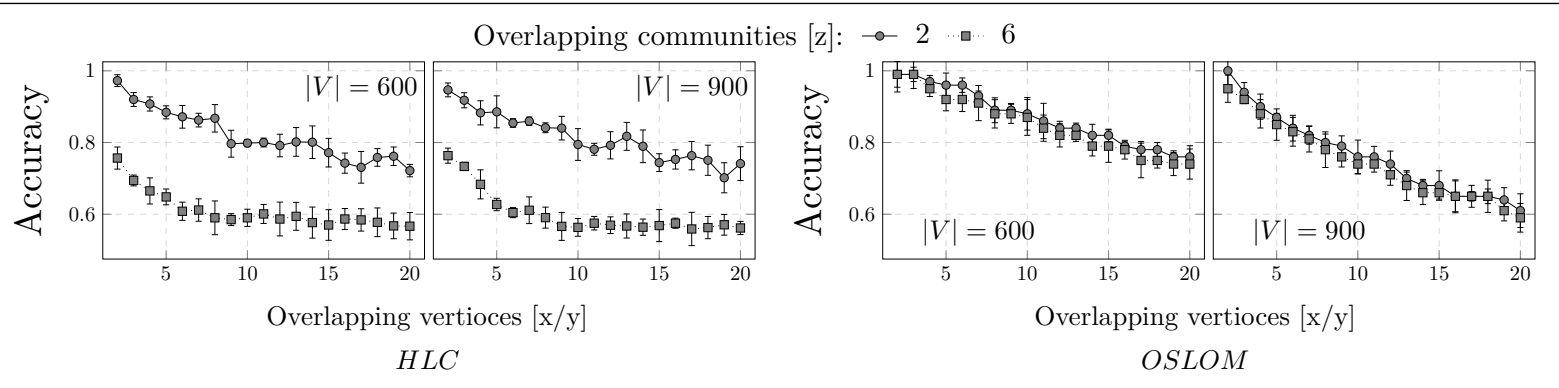

(a)

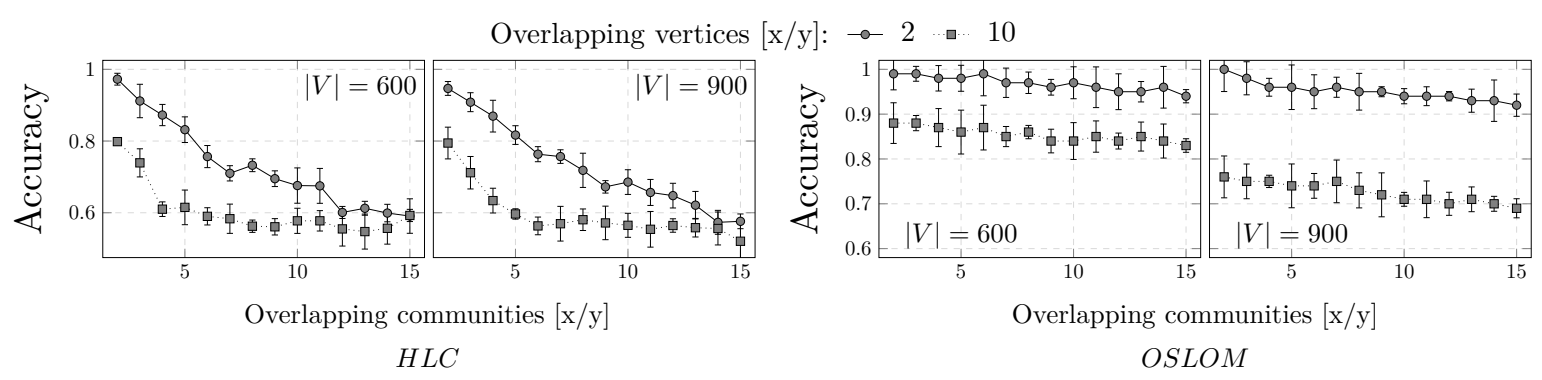

(b)

Figure 44 - Acc values for HLC (left) as OSLOM (right) as a function of the numbers of overlapping vertices (a) and overlapping communities (b) in 532 synthetic weighted bipartite networks.

Source: Elaborated by the author.

$x=y \geq 10$, which suggests its performance is not strongly affected by the number of overlapping vertices. On the other hand, parameter $z$ potentially impacted the quality of the solution, since $z=2$ (upper curves) yielded higher accuracies than $z=6$ (bottom curves). The upper and bottom curves differ to up 0.15 , although their difference decreases as $x$ increases. This effect is shown in Figure 44b (left) and confirms Acc decreases quickly as $z$ increases. Therefore, whilst the influence of parameters $x$ and $y$ on algorithm performance is unclear, it is surely strongly affected by the presence of overlapping communities.

Figure 44a (right) shows the Acc values achieved by OSLOM decrease as the number of overlapping vertices increases, which suggests the algorithm's performance is strongly affected by this property. On the other hand, the choice of parameter $z$, which determines the number of overlapping communities, seems to exert a limited impact on solution quality, since curves for $z=2$ and for $z=6$ are nearly overlapping, i.e., yielded similar accuracies. This effect is shown in Figure 44b (right) and confirms that Acc values remain stable as $z$ increases.

Results are amenable to statistical analysis, since the algorithms were evaluated in 802 representative and independent networks (270 and 532 networks were used, respectively, to evaluate their performance by means of NMI and Acc). Therefore, a Nemenyi post-hoc test (DEMSAR, 2006) was applied to the results depicted in Figures 43 and 44 to detect statistical differences in the performances of both algorithms. According to the Nemenyi statistics, the critical value for comparing the mean-ranking of two different algorithms at 95 percentile is 0.12. Mean-ranking differences above this value are significant. The average ranks of HLC and 
OSLOM were 1,0 and 2,0, respectively, therefore, OSLOM yielded in statistically higher values than HLC.

The execution times of HLC and OSLOM were measured to analyze the impact of network properties such as size and density on algorithm scalability. The runtime is primarily affected by the total number of vertices, rather than by the layer sizes, therefore, we generated 1,530 weighted bipartite networks, varying $|V|$ within the range $[100,1000]$ at increments of 100 , so that $v=\left[v_{1}, v_{2}\right],|V|=v_{1}+v_{2}$ and $v_{1}=v_{2}$ (equal sized layers). Parameter $d$ varied within the range $[0.1,0.9]$ at increments of 0.05 and parameter $n$ varied within the range $[0.1,0.3]$ at increments of 0.025. The remaining parameters were set as $x=y=0.05|V|, z=0.01|V|$ and $c=0.02|V|$. Figure 45 shows runtime of both HLC (left) and OSLOM (right) as a function of the parameters controlling dispersion, or edge density (parameter $d$ in Figure 45a), size (parameter $v$ in Figure 45b) and noise (parameter $n$ in Figure 45c).

As far as HLC is concerned, Figure 45a (left plot) indicates a linear correlation between runtime and dispersion values and Figure 45b (left) depicts a nearly quadratic relation with network size. Indeed, an increase in the number of vertices implies an exponential increase in the total number of edges and higher values of the dispersion parameter directly impact on the network edge density. According to Figure 45c (left), increasing noise does not affect runtime. Therefore, the combination of network density and network size strongly affects the performance of HLC.

As far as OSLOM is concerned, no clear correlation between runtime and edge density is observed in Figure 45a (right plot). Indeed, OSLOM has quadratic complexity in the number of vertices $|V|$, observable in Figure 45b (right), whereas an increase in dispersion parameter $d$ directly impacts on the number of edges, without affecting the asymptotic convergence of the algorithm. Furthermore, Figure 45c (right) suggests increasing noise levels significantly impacts runtime. We conclude the execution time of OSLOM is strongly impacted by a combination of larger network sizes and high noise levels.

The previous analyses reveal that benchmarking networks obtained with BNOC uncover interesting information on the accuracy and runtime behavior of both algorithms, in relation with network properties such as size, density, noise and overlapping levels. The analyses also enabled a statistical comparison of their performances.

\subsubsection{Comparison with existing benchmarks}

We turned off the overlapping community settings in BNOC in order to reproduce the synthetic scenarios considered in previous studies by Melamed (2014) and Larremore, Clauset and Jacobs (2014). Our goal is to assess the reliability of the networks synthesized by BNOC, verifying whether the tool can reproduce networks displaying structural properties similar to those already considered in the literature. 
Chapter 3. A benchmarking tool for the generation of bipartite network models with overlapping 106 communities

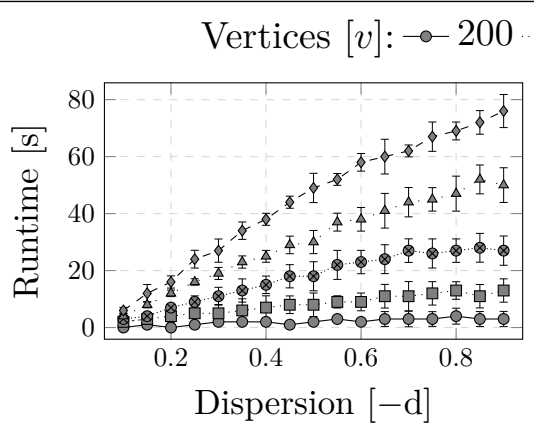

$H L C$

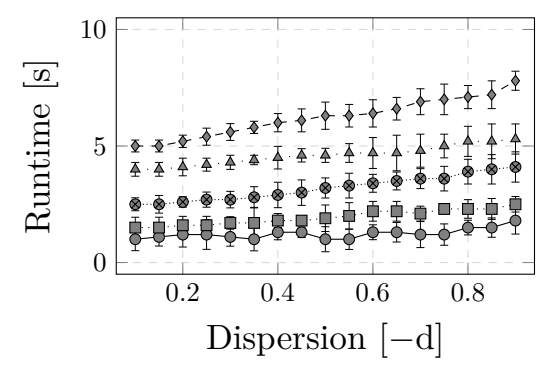

$O S L O M$

(a)

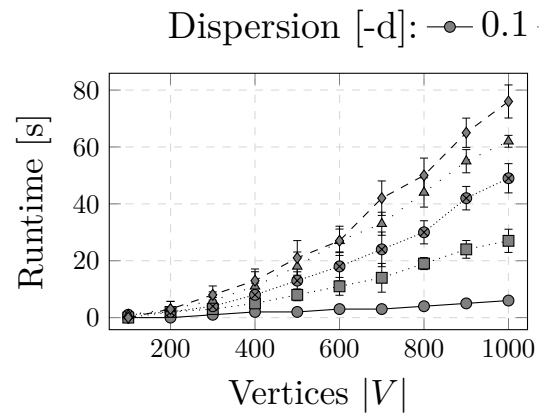

$H L C$
$0.3-0.5 \sim 0.7-0.9$

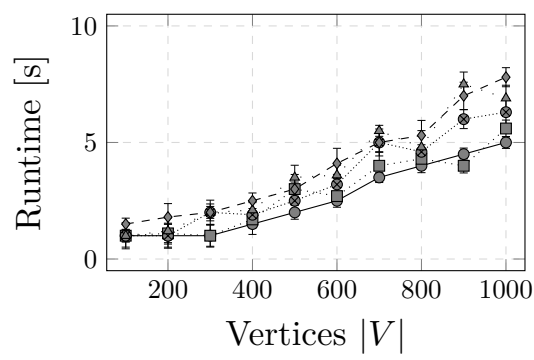

$O S L O M$

(b)

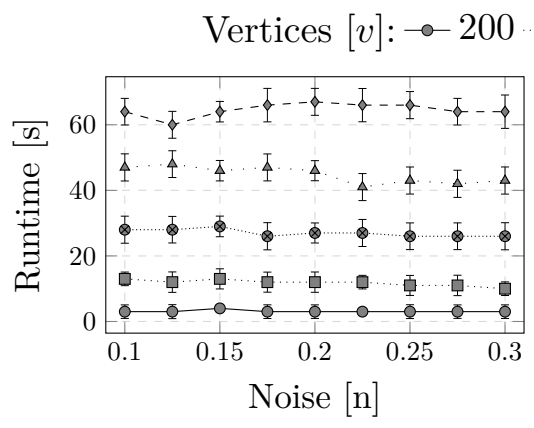

$H L C$
$400 \cdots 600 \sim 800-\triangle 1000$

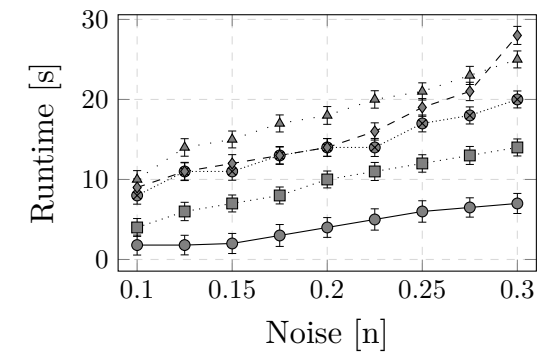

$O S L O M$

(c)

Figure 45 - HLC and OSLOM runtimes, as a function of network density a, number of vertices $b$ and amount of noise $\mathrm{c}$.

A first study considered the synthetic networks described in Melamed (2014), henceforth called Melamed networks. That study manipulated two properties of the networks: (i) the number of communities in each layer: two networks were built with three communities in both layers and two networks with three and two communities in the first and second layer, respectively; (ii) the number of vertices: two networks were built with $v=[60,120]$ and two networks with $v=[600,1200]$.

The networks have been synthesized in BNOC using the following parameter settings: (1) $v=[60,120], c=[3,3], n=0.2, d=0.6, p=0.6$; (2) $v=[600,1200], c=[3,3], n=0.1$, $d=0.4, p=0.4$; (3) $v=[60,120], c=[3,2], n=0.5, d=0.5, p=0.5$; and (4) $v=[600,1200]$, 
$c=[3,2], n=0.3, d=0.3, p=0.3$.

Figures 46a-46d illustrate the four Melamed networks; Figures 46e-46h illustrate the corresponding BNOC counterparts. The network visualizations allow a global observation of the topological similarities between the pairs of synthetic networks thus obtained. The BNOC networks closely resemble the Melamed networks, particularly the pairs constructed with the same number of communities in both layers, namely a-e and b-f. Layouts in Figure 46 have been computed with the Fruchterman-Reingold force-directed algorithm under same parameter settings (FRUCHTERMAN; REINGOLD, 1991).

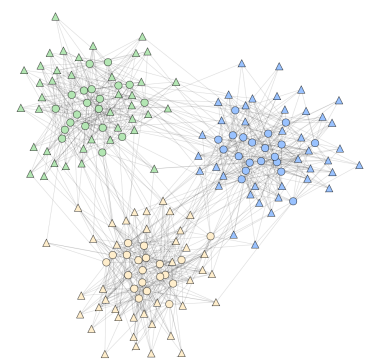

(a)

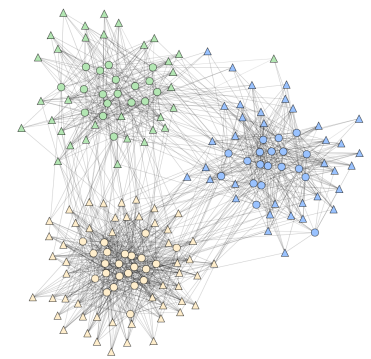

(e)

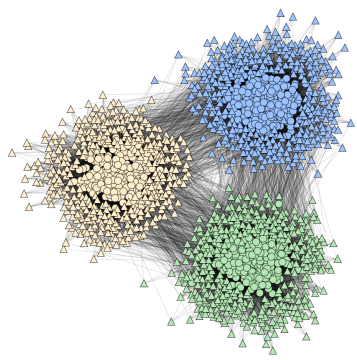

(b)

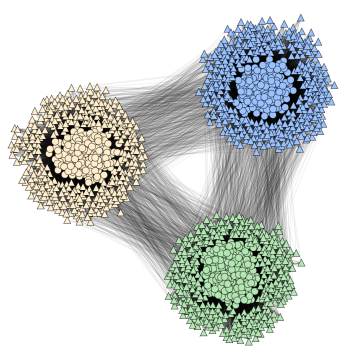

(f)

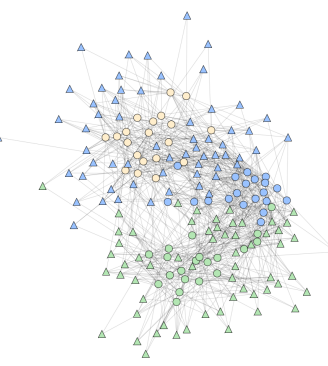

(c)

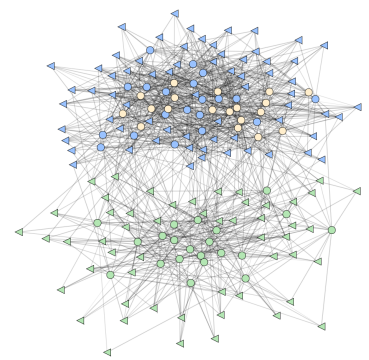

(g)

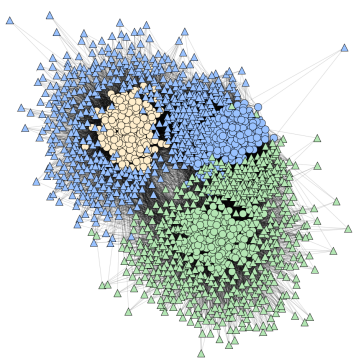

(d)

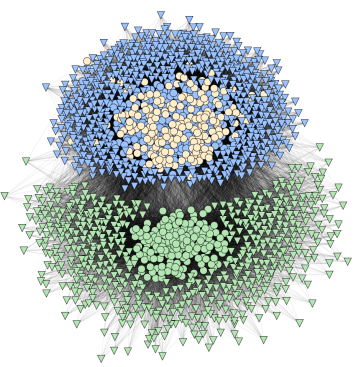

(h)

Figure 46 - Two equivalent sets of synthetic networks obtained with different benchmarking tools. (a), (b), (c), and (d) display the set of Melamed networks; and (e), (f), (g), and (h) display the corresponding networks synthesized using BNOC.

Source: Elaborated by the author.

In order to obtain an objective estimate of their topological equivalence we evaluated the well-known community detection algorithm biSBM ${ }^{10}$ (LARREMORE; CLAUSET; JACOBS, 2014) on both sets of networks. Table 8 reports the mean normalized mutual information (NMI) (DANON et al., 2005) measures and corresponding standard deviation errors obtained with 10 network replications and 10 biSBM executions. The results obtained are highly similar, providing strong evidence that the network pairs in Figure 46 - a-e, b-f, c-g and d-h - have equivalent topological properties.

A similar analysis was conducted in the synthetic networks provided in Larremore, Clauset and Jacobs (2014), henceforth called Larremore networks. That study considered two scenarios: The first typifies an easy case for community detection, of networks with $v=[1000,1000]$ 
Chapter 3. A benchmarking tool for the generation of bipartite network models with overlapping 108 communities

Table 8 - Normalized mutual information (NMI) mean values and standard deviations resulting from running biSBM in the BNOC and the Melamed network sets.

\begin{tabular}{lrrrr} 
Benckmark & Network A & Network B & Network c & Network D \\
\hline BNOC Networks & $0.80 \pm 0.03$ & $0.97 \pm 0.01$ & $0.85 \pm 0.03$ & $0.98 \pm 0.01$ \\
Melamed Networks & $0.79 \pm 0.02$ & $0.98 \pm 0.02$ & $0.85 \pm 0.02$ & $0.98 \pm 0.01$
\end{tabular}

Source: Research data.

and four equal sized communities. The second represents a more challenging scenario, where the networks were built with $v=[300,700]$ and vertices split into $\{100,150,50\}$ and $\{350,350\}$ communities in the first and the second layers, respectively. Each scenario is represented by 20 networks obtained varying the mixing parameter $\gamma$ within the range $[0,1]$ at increments of 0.05 , wherein $\gamma=0$ denotes the maximum setting for noise and $\gamma=1$ denotes the absence of inter-community edges.

The first set of networks was replicated employing the following parameter settings in BNOC: $d=0.25, v=[1000,1000], c=[4,4], p^{0}=p^{1}=[0.25,0.25,0.25,0.25]$; for the second one the settings were: $v=[300,700], c=[3,2], p^{0}=[0.4,0.3,0.2]$ and $p^{1}=[0.5,0.5]$. Again, for each case 20 networks were created with noise level parameter $n$ varying in the range $[0,1]$ at increments of 0.05 .

There are two major differences in the processes used to synthesize the Larremore and the BNOC networks. First, the generating model in Larremore, Clauset and Jacobs (2014) creates the communities by adding an exact number of vertices to each community, whereas BNOC considers the probabilities of vertices belonging to each community. Second, the mixing parameter $\gamma$, used to control noise in (LARREMORE; CLAUSET; JACOBS, 2014), is the opposite of how noise is defined in BNOC.

Figure 47 reports the corresponding biSBM NMI mean values and standard deviations (y-axis) obtained, as a function of $\gamma$ (upper x-axis) or $n$ (lower x-axis) for both network sets, with a depicting the easy scenario and $b$ depicting the difficult one. Similarly to the previous study, similar NMI behavior is observed in the synthetic network sets, i.e., the curves in both the easy and the difficult scenarios display equivalent behavior. In summary, for very low noise levels (indicated by $n$ or $\gamma$ ) they decrease slowly as noise increases; then the curves drop sharply as the noise levels increase further and the number of inter-community edges approaches $50 \%$; finally NMI stabilizes at very low values on the noisier networks.

The previous analyses confirm BNOC as a reliable general-purpose benchmarking tool useful to replicate network models already considered in the literature or to synthesize new models with arbitrary properties. 


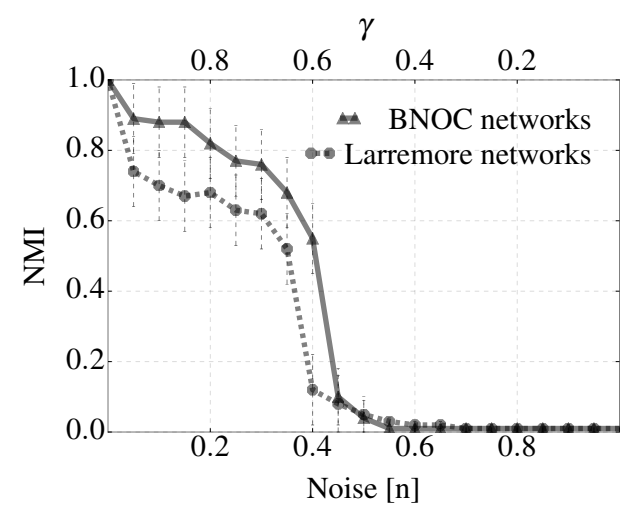

(a)

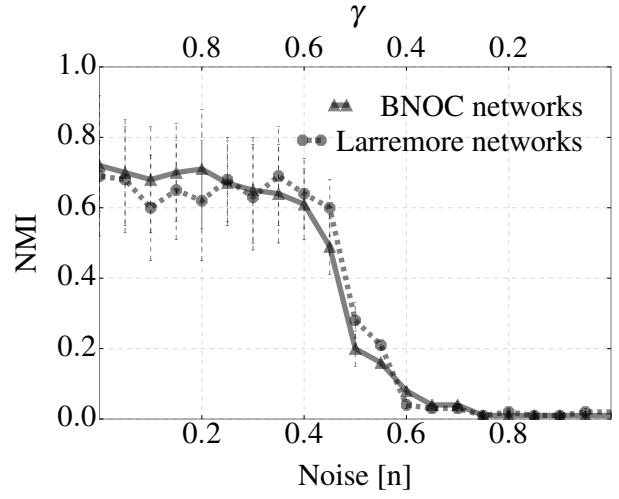

(b)

Figure 47 - NMI values for biSBM as a function of mixing parameter $\gamma$ (lower $\mathrm{x}$-axis) or noise level parameter $n$ (upper x-axis) obtained on the Larremore and the BNOC networks. (a) shows results for the easy scenario; (b) shows results for the difficult scenario. Each scenario is described by 20 networks (varying noise levels) and mean values and errors are relative to 10 network replications and 10 biSBM executions.

Source: Elaborated by the author.

\subsection{Conclusion}

In this paper we introduced BNOC, a tool to generate synthetic weighted bipartite networks with overlapping structures for benchmarking purposes.

Its potential was illustrated with an assessment of two well-known overlapping community detection algorithms, HLC and OSLOM, on benchmark networks with very distinctive properties. Our results indicate the performance of HLC is highly sensitive to both noise and number of overlapping vertices and communities. Furthermore, its execution times are strongly affected by network size and community density. On the other hand, the performance of OSLOM is highly sensitive to edge density and number of overlapping vertices and its execution times are strongly affected by both network size and noise levels. Such findings deserve attention in further studies and application of these algorithms.

This study also illustrates BNOC's potential usefulness as a flexible, robust and reliable resource for creating arbitrary bipartite networks for benchmarking purposes. It can synthesize large-scale bipartite networks with tens or even hundreds of thousands vertices and tens of millions edges in very reasonable times. We observed, however, that scalability may be impaired when creating networks that lack a community structure or have very few communities.

We also compared BNOC with existing benchmarking models. The results of comparing the synthetic models obtained from different tools provide strong evidence on the reliability of BNOC as a general-purpose benchmarking tool for creating networks with arbitrary topological properties.

BNOC may be useful for benchmarking other kinds of algorithms and applications 
Chapter 3. A benchmarking tool for the generation of bipartite network models with overlapping

beyond community detection. For instance, if the layers represent, respectively, observations and features, and communities are interpreted as classes, it may support comparative assessment of classification algorithms. It may support the design very large networks with well-known target properties for purposes of testing e.g., graph drawing or link prediction algorithms, since topological features such as community structure and membership can be imposed a priori to reflect desired characteristics.

As future work we intend to extend the tool to handle directed bipartite networks. Additional issues deserve further investigation, including: conducting systematic studies on how to tune the parameter values to yield networks exhibiting a particular combination of target features; in-depth benchmarking of algorithm behavior in networks with highly unbalanced layers; an analysis of edge weights on distinct ranges in setting the corresponding probability parameters $s$ and $d$. The current implementation of BNOC can be downloaded at <https://github. com/alanvalejo/bnoc $>^{11}$.

\section{Appendix}

\section{A HNOC: Extension to $k$-partite and heterogeneous networks}

As in the case of bipartite networks there is a lack of benchmarking tools to create $k$-partite and heterogeneous networks for assessing community detection and other algorithms. $k$-partite networks have $k$ vertex types, rather than just two, with edges connecting only vertices of different types. In heterogeneous networks this second restriction is dropped, i.e., edges can occur between vertices of the same type. Since they are direct generalizations of bipartite networks, we extended BNOC to synthesize general heterogeneous networks. This extension, called HNOC, can be useful to generate HIN models to support development and validation of new methods. HNOC inherits BNOC's major features as a flexible and robust resource to synthesize a variety of benchmarking networks with distinct properties in reasonable times.

A heterogeneous information network (HIN) (SHI et al., 2017b) consists of $m$ disjoint sub-sets of vertices of different types (called layers) and edges connecting these elements. A network $\mathscr{X}$ with $m$ vertex types can be partitioned into sub-sets $X_{i}=\left\{x_{i, 1}, \ldots, x_{i, n_{i}}\right\}$ for each type $i$. A HIN is represented as a graph $G=(V, E, W)$, where $V=\bigcup_{i=1}^{m} X_{i}$ with $m>2, E$ is the set of edges, and $W$ is the set of edge weights.

HINs have a inherently complex structure that can be difficult to handle and visualize. The structure of connections can be described by a network schema (SHI et al., 2017b), which defines a meta template for the network that describes its vertex and connection types. Given a

11 The tool will be made available immediately after paper acceptance. 
graph $G$, its network schema, denoted $T_{G}(\mathscr{A}, \mathscr{R})$, is a directed graph defined over element types $\mathscr{A}$ with edges as relations from $\mathscr{R}$, obtained via mapping functions $\varphi: V \rightarrow \mathscr{A}$ and $\psi: E \rightarrow \mathscr{R}$, respectively. Figure 48 exemplifies network schemas for a bipartite network, a $k$-partite network and an heterogeneous network.

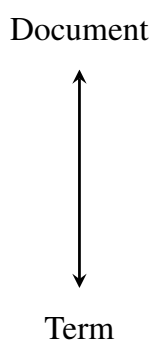

(a)

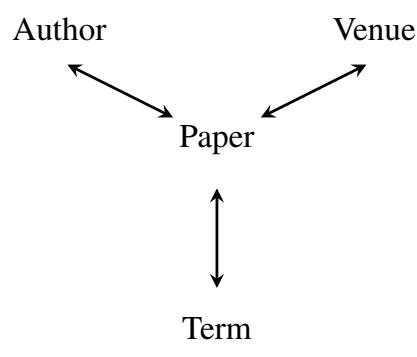

(b)

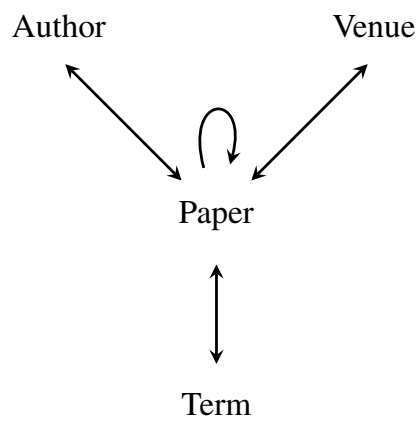

(c)

Figure 48 - Network schemas: (a) bipartite network schema; (b) $k$-partite network schema; and (c) heterogeneous network schema.

Source: Elaborated by the author.

The schema explicitly identifies the $m$ vertex types and the $r$ connection types. Each connection type is described by the types of the two endpoints and the connection meaning, since pairs of entities can admit multiple types of connections, as in the case of multi-relational networks (ZHONG et al., 2013). Thus, a HIN can be interpreted as a composition of $r$ bipartite (or homogeneous, if both endpoints of the connections are of the same type) networks, as illustrated in Figure 49.

Following this interpretation we extended BNOC to generate synthetic heterogeneous networks. The extension iterates over each pair of vertex and connection types specified in the network schema, employing similar steps to build the communities in each iteration:

1: Execute $m$ iterations of BNOC's Steps 1 and 2 to build each layer $i$ with $V_{i}$ vertices, set a single community on each layer and introduce the overlapping structures.

2: For each pair of layers specified in the schema, execute BNOC's Steps 3, 4 and 5 in order to establish the specified connections, weights, density and noise levels.

Since heterogeneous networks have multiple layers and multiple connection types, the extension required modifying some BNOC parameters and introducing a few additional parameters, as described in Table 9. The following parameters were added: the number of layers $m$ and the set connected layers, henceforth called "schema", e. Furthermore, the dispersion and noise parameters must be defined for each schema, since each iteration handles a pair of connected layers. 
Chapter 3. A benchmarking tool for the generation of bipartite network models with overlapping

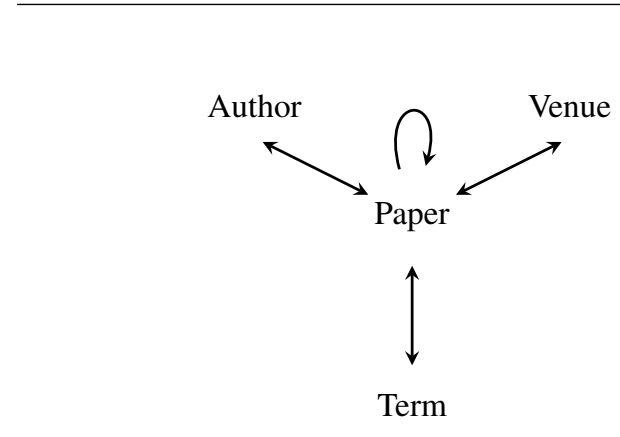

(a) Network

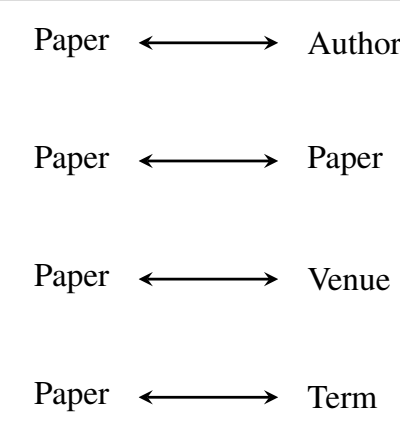

(b) Connections

Figure 49 - A HIN described as $r$ bipartite/homogeneous networks.

Source: Elaborated by the author.

Table 9 - Modified and additional parameters in HNOC.

\begin{tabular}{lrrrl} 
Parameter & Type & Domain & Default & Description \\
\hline -m, -layers & number & $\mathbb{N}$ & 3 & Number of vertex types \\
-v, -vertices & $|p|=m$ & $(0,|V|] \in \mathbb{N}$ & {$[10,10,10]$} & Number of vertices for each layer \\
-e, -schema & $|p|=r \times 2$ & $\mathbb{N}$ & null & List of pairs that defined the connected layers \\
-p, -probabilities & $|p|=m \times c$ & $(0,|V|] \in \mathbb{R}$ & null & Probabilities for vertices in each layer for each community \\
-d, --dispersion & $|p|=m$ & $\mathbb{R}_{+}$ & 0.1 & Dispersion of negative binomial distribution for each scheme \\
-n, --noise & $|p|=m$ & $(0,1] \subseteq \mathbb{R}$ & 0.01 & Noise for each scheme \\
-b, -balanced & boolean,,$|p|=m$ & 0,1 & {$[0,0,0]$} & Boolean balancing flag that suppresses parameter -p \\
-x, -overlap & $|p|=m$ & $(0,|V|] \in \mathbb{N}$ & {$[0,0,0]$} & Number of overlapping vertices in each layer \\
-z, -noverlap & $|p|=m$ & $(0,|c|] \in \mathbb{N}$ & {$[2,2,2]$} & Overlapping communities in each layer \\
& & & Source: Research data.
\end{tabular}

Figure 50 illustrates two networks created with distinct parameter combinations. Unless informed otherwise the parameter settings correspond to the default values informed in Tables 7 and 9. Figure 50a depicts a 4-partite network with some community overlapping.

The network was built so that all layers have the same number of communities, the probability set to produce balanced communities, different numbers of overlapping vertices in each layer, and the same dispersion (edge density) $d$ in all pairs of connected layers. Figure $50 \mathrm{~b}$ illustrates a 3-partite network with heterogeneous structure and edges between vertices of the same type in one of the layers. The example can describe a hypothetical author-paperterm network, in which authors are connected with their papers, terms are connected with their neighbouring terms in the text and with the papers in which they appear. The upper left, central and upper right layers, represent, respectively, the Term, Paper and Author entities. The network has been created so that the connections between the different pairs of entities display different patterns, e.g., there is a dense topology of connections between terms and papers, whereas connections between terms and terms, or between authors and papers are sparser. The schema is inspired in the largely used real-world data of DBLP ${ }^{12}$ (Digital Bibliography \& Library Project), 
a computer science bibliographic dataset that relates documents, authors and terms.

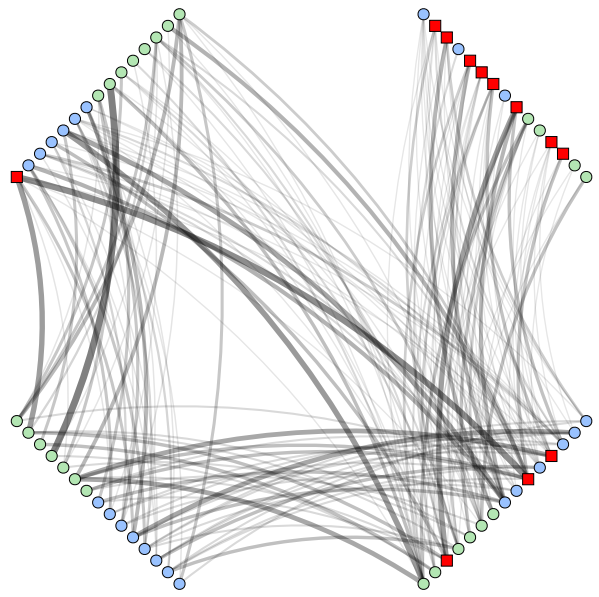

(a)

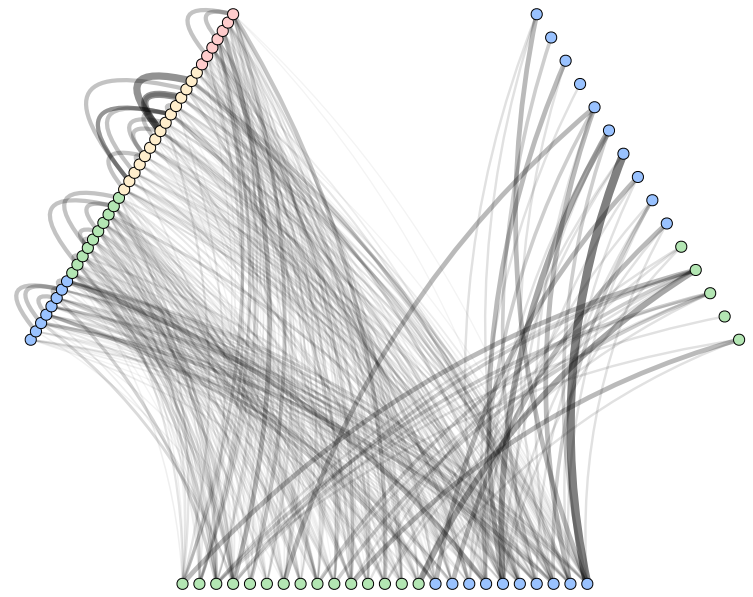

(b)

Figure 50 - Heterogeneous networks generated with HNOC presenting distinct topological structures and properties: red squares depict overlapping vertices and colored circles indicate non-overlapping vertices and their assigned community; line widths reflect the corresponding edge weights. (a) illustrates a 4-partite network with $v=[15,15,15,15]$, $e=[(0,1),(1,2),(2,3),(3,1)]$ and $x=[8,3,0,1]$; (b) depicts a heterogeneous network obtained with settings $v=[40,25,15], e=[(0,1),(1,2),(2,2)], c=[2,2,4]$, and $d=$ $[0.45,0.85,0.15,0.15]$. The network drawings were obtained based on the technique described by Uslu and Mehler (2018).

Source: Elaborated by the author. 

CHAPTER

\section{4}

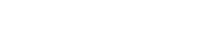

\section{COMMUNITY DETECTION IN BIPARTITE NETWORK: A MODIFIED COARSENING APPROACH}

Interest in algorithms for community detection in networked systems has increased over the last decade, mostly motivated by a search for scalable solutions capable of handling large-scale networks. Multilevel methods provide a potential solution to scalability, as they reduce the cost of a community detection algorithm by applying it to a coarsened version of the original network. The solution obtained in the small-scale network is then projected back to the original large-scale model to obtain the desired solution. However, standard multilevel methods are not directly applicable to bipartite networks, and there is a gap in the existing literature on multilevel method applied to such networks. This article addresses this gap and introduces a novel multilevel method based on one-mode projection, which allows executing classical and well-known multilevel methods in bipartite networks. The approach has been validated with an algorithm for community detection that solves the Barber's modularity problem. We show it can scale a computationally expensive target algorithm to handling larger networks, while preserving solution accuracy.

\subsection{Introduction}

Complex networks are relational structures that represent many real-world systems formed by a large number of highly interconnected dynamical units. Many such systems exhibit a natural bipartite (or two-layer) structure, in which the set of units (known as vertices) is split into two disjoint subsets (or layers) and connections (known as edges) are established between units placed in different layers. Document-word (ROSSI; LOPES; REZENDE, 2016), proteinligand (JEONG et al., 2000) and actor-movie (WATTS; STROGATZ, 1998) networks are a few examples of real-world bipartite networks. 
Many such networks present community structures. They are defined as groups of vertices densely connected to each other within a group and sparsely connected to vertices in other groups. Such structures are important for characterizing the network behavior, as vertices that belong to the same community may share common properties or play similar roles in a networked system. Therefore, the identification of community structures in networked systems contributes to a better understanding of their topological structure and dynamical processes (FORTUNATO, 2010). For instance, communities in a biological protein network typically correspond to proteins that share a single specific function (MAHMOUD et al., 2014). Furthermore, the increasing interest in identifying community structures in bipartite networks (DORMANN; STRAUSS, 2013; THÉBAULT, 2013; LARREMORE; CLAUSET; JACOBS, 2014; DORMANN; STRAUSS, 2014; ALZAHRANI; HORADAM, 2015; BECKETT, 2016) is a reliable indicator of this being a relevant research topic.

Community detection algorithms aim at subdividing a set of vertices into $k$ communities while minimizing the number of edges connecting vertices placed in different communities (FORTUNATO, 2010; VALEJO et al., 2014). This is a hard combinatorial optimization problem, in which the goal is to optimize a given cost function, such as modularity (GIRVAN; NEWMAN, 2002). Searching for an optimal solution on large-scale networks is unfeasible, since the number of possible solutions can be exponential.

To overcome this problem, researchers resorted to multilevel methods, in which: an original network is gradually coarsened at multiple levels by collapsing vertices and edges (coarsening phase); an initial community structure is obtained on the coarsest network (solution finding phase); and the initial solution is successively projected back over the inverse sequence of coarsened networks, until the original network (uncoarsening phase).

Many multilevel community detection algorithms have been developed for unipartite (or one-mode) networks. Some studies introduced multilevel community detection methods targeted at specific types of networks; e.g., Abou-Rjeili and Karypis (ABOU-RJEILI; KARYPIS, 2006) considered networks with a power-law degree distribution, and Valejo et al. (VALEJO et al., 2014a; VALEJO; VALVERDE-REBAZA; LOPES, 2014a) explored properties of social networks, such as high transitivity and assortativity. Other contributions focused on applying multilevel method to improve a measure of modularity (DJIDJEV, 2008; SCHUETZ; CAFLISCH, 2008a; YE; HU; YU, 2008; NOACK; ROTTA, 2009; ROTTA; NOACK, 2011; DJIDJEV; ONUS, 2013; LASALLE; KARYPIS, 2015). Furthermore, many authors investigated parallel paradigms to speed up the coarsening and refinement phases (BAÑOS et al., 2004; BANOS et al., 2004; TRIFUNOVIC; KNOTTENBELT, 2004b; TRIFUNOVIC; KNOTTENBELT, 2004a; ERCIYE; ALP; MARSHALL, 2005; SCHWEITZ; AGRAWAL, 2007; LASALLE; KARYPIS, 2013; LASALLE; KARYPIS, 2015).

Nonetheless, the aforementioned approaches cannot be directly applied to bipartite networks, since standard coarsening algorithms operate by matching and collapsing pairs of 
connected vertices. Such solutions presume all vertices are alike, i.e., represent the same type of entity. However, vertices in different layers of bipartite networks are not connected, and typically represent entities of different types. Coarsening such networks requires a different treatment of the vertices, as only vertices of the same type (i.e., in the same layer, and thus unconnected) should be matched.

In a previous study (VALEJO et al., 2017) we addressed this gap and introduced a novel one-mode projection-based multilevel method that enables applying standard coarsening algorithms to bipartite networks. In this paper, we extend our previous study in the following manner: (1) we describe the proposed model in further detail; (2) we evaluate the application of another popular matching algorithm in this context, now contemplating an analysis of three matching algorithms; (3) we extend the statistical analysis of the quality of the results yielded by the proposed multilevel solution; (4) we expand the analysis of the scalability of the multilevel solution. Our empirical investigation on an expressive set of synthetic network models has shown that our multilevel solution can be combined with a community detection algorithm, yielding expressive speedups with no significant loss in solution quality.

The remainder of the paper is organized as follows: Section 4.2 reviews some basic concepts on networks and provides a brief overview of standard multilevel methods; Section 4.3 introduces the proposed multilevel formulation for bipartite networks and its implementation; Section 4.4 presents results from an empirical study on a large synthetic test suite; finally, Section 4.5 summarizes the results and discusses future work.

\subsection{Fundamentals}

This section describes the terminology and fundamental concepts required to understand the proposed solution.

\subsubsection{Basic definitions}

A unipartite network is given by $G(V, E, \omega)$, where $V=\left\{v_{1}, v_{2}, \ldots, v_{n}\right\}$ is the set of vertices, $E=\left\{e_{1}, e_{2}, \ldots, e_{k}\right\}$ is the set of edges connecting vertices, such that $e_{i}=(v, u)=$ $\{(u, v)=(v, u) \mid u, v \in V\}$ and $\omega=\left\{w_{1}, w_{2}, \ldots, w_{k}\right\}$ is the set of weights, so that each $w_{i} \in \mathbb{R}$ is associated with a corresponding edge $e_{i}$. Two vertices are said to be neighbors if they are connected by at least one edge.

A bipartite network is given by $G(V, E, \omega)$, where $V$ is partitioned into two sets $V^{1}$ and $V^{2}$ so that $V^{1} \cap V^{2}=\emptyset, V^{1}=\left\{u_{1}, u_{2}, \ldots, u_{n}\right\}$ is a set (or layer) of vertices, $V^{2}=\left\{v_{1}, v_{2}, \ldots, v_{m}\right\}$ is another set of vertices and $E=\left\{e_{1}, e_{2}, \ldots, e_{k}\right\}$ is the set of edges connecting vertices in different layers, i.e. for all $(u, v) \in E, u \in V^{1}$ and $v \in V^{2}$ and $E \subseteq V^{1} \times V^{2}$. Similarly, $\omega=\left\{w_{1}, w_{2}, \ldots, w_{k}\right\}$ is the set of edge weights. Figure 51 illustrates a bipartite network. 


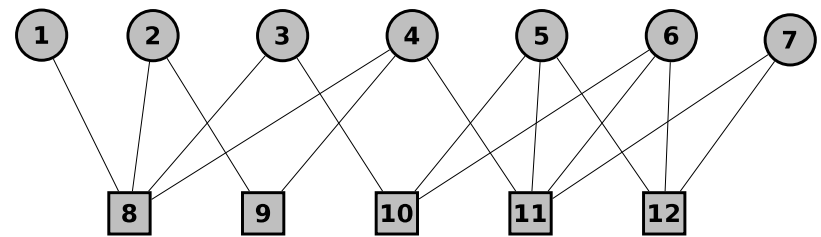

Figure 51 - A bipartite network.

Source: Elaborated by the author.

Bipartite networks can be transformed into unipartite networks through a one-mode projection (NEWMAN, 2001c; OPSAHL, 2010; PADRóN; NOGALES; TRAVESET, 2011). Applying a one-mode projection to a bipartite network generates two unipartite networks, one for each layer, $G^{1}$ and $G^{2}$, so that vertices with common neighbors are connected by edges in their respective projection. Figure 52 illustrates the result of applying such a procedure to the bipartite network shown in Figure 51.

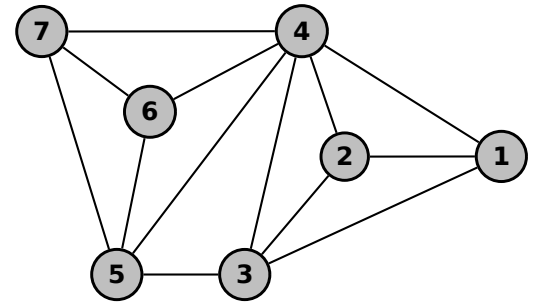

(a) $G^{1}$

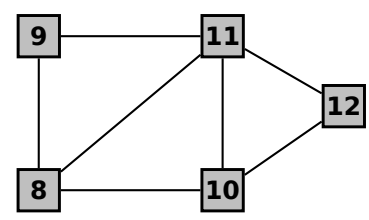

(b) $G^{2}$

Figure 52 - Unipartite networks $G^{1}$ and $G^{2}$ resulting from a one-mode projection of the bipartite network in Figure 51.

Source: Elaborated by the author.

Figures 52a and 52b show, respectively, the one-mode projections of $V^{1}$ (i.e., $G^{1}$ ) and $V^{2}$ (i.e., $G^{2}$ ). If two vertices share multiple common neighbors, their connection in the unipartite projection should reflect this topology. In weighted unipartite projections, edge weights are assigned according to the number of common neighbors between the two connected vertices, as illustrated in Figure 53 for the particular pair of projected vertices $\{4,5\}$.

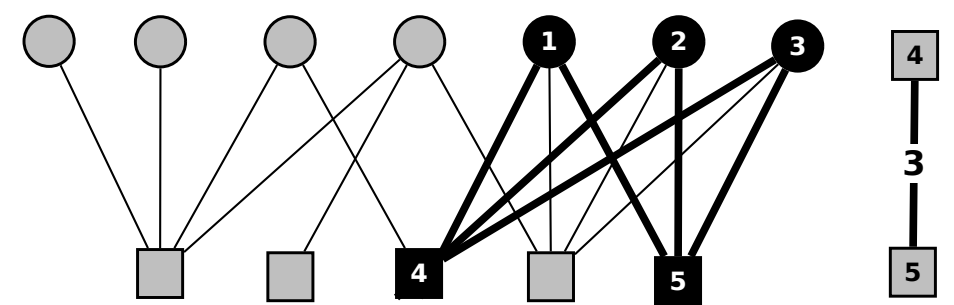

Figure 53 - A weighted projection: vertices 4 and 5 are connected by an edge of weight 3 , as they share three neighbors in common.

Source: Elaborated by the author. 


\subsubsection{Multilevel method}

Multilevel method reduces the number of operations required to solve a target problem, i.e, the multilevel method can execute a complex algorithm, that can not be executed on a very large network, on a coarsened (also called reduced) version of this network which requires a much smaller number of operations. The results obtained in the reduced network are then projected back to obtain the actual solution for the original network.

Let us consider an initial unipartite network $G_{0}\left(V_{0}, E_{0}, \omega_{0}\right)$ and assume its size (in terms of edges and vertices) prevents the execution of a target algorithm. A multilevel method could be applied as follows (KARYPIS; KUMAR, 1998a):

Coarsening phase: Original network $G_{0}$ is transformed into a sequence of coarsened networks $G_{1}, G_{2}, \cdots, G_{L}$, wherein $G_{L}$ is the coarsest network. The size of the vertex set of each subsequent network is reduced at each iteration, i.e., $\left|V_{0}\right|>\left|V_{1}\right|>\left|V_{2}\right|>\ldots>\left|V_{L}\right|$.

Solution finding phase: The target algorithm (or an computationally expensive task) is applied to coarsest network $G_{L}$, thus generating a initial solution. As $\left|V_{L}\right|$ is sufficiently small, the target algorithm can be executed in feasible time. In the present study, we consider a community detection algorithm as the target.

Uncoarsening phase: The initial solution obtained in the coarsest network $G_{L}$ is projected back, through the intermediate levels $G_{L-1}, G_{L-2}, \cdots$, until the original network $G_{0}$, thus generating the final solution.

The coarsening phase is an iterative process that constructs a sequence of reduced versions of the initial network $G_{0}$. The vertices of a network $G_{l}$ are collapsed into super-vertices to obtain a network $G_{l+1}$. Edges incident to the original vertices are collapsed to obtain the edges incident to a super-vertex. The coarsening process is split into two phases, namely matching and contraction.

In the matching phase, edges, or vertex pairs, are selected to be collapsed. Once an edge in $G_{l}$ has been selected, its incident vertices are candidates to form a super-vertex. Vertices from $G_{l}$ with no incident edge selected are inherited by $G_{l+1}$. In the present study, we consider three well-known matching methods, introduced by Karypis and Kumar (1998a), namely:

Random matching $(R M)$ : Vertices are visited in random order. If a vertex $v$ has not been matched yet, one of its unmatched neighbors is selected. If such a vertex $u$ exists, the pair $(v, u)$ is included in the matching set, otherwise $v$ remains unmatched. Although it may yield poor results, $R M$ is very fast and straightforward.

Heavy edge matching $(H E M)$ : Similarly to $R M$, vertices are also visited in random order. However, unlike $R M$, vertices $v$ and $u$ are matched if edge $(v, u)$ has maximum weight over 
all valid edges incident to $v$. Although $H E M$ does not guarantee the matching obtained has maximum weight, it yields better results than $R M$ with equivalent asymptotic complexity.

Greedy Heavy Edge Matching (GHEM): Edges are sorted by descending weight and then visited iteratively. For each visited edge $(u, v)$, if neither $u$ nor $v$ is matched yet, the pair $\{u, v\}$ is included in the matching. The algorithm is near linear time and although slightly more expensive than $R M$ and $H E M$, it is more robust in practice.

Next, the contraction algorithm builds the coarsened network directly from the matching by collapsing each pair of matched vertices into a single super-vertex $(s \mathrm{~V})$. Edges incident to $s V \in V_{l+1}$, called super-edges, are obtained by collapsing edges incident to vertices $\{u, v\} \in V_{l}$. The weight of the super-edge is given by the sum of the weights of all edges incident to $\{u, v\} \in V_{l}$.

The target algorithm (community detection, in this case) is then evaluated in the coarsest network $G_{L}$ to obtain an initial solution. As $\left|V_{L}\right| \ll\left|V_{0}\right|$, the algorithm converges faster and can generate an initial solution in feasible time.

In the uncoarsening phase, the initial solution is successively projected back to $G_{0}$. At each level, each super-vertex $s V=\{u, v\} \in V_{l+1}$ is expanded to its original vertices in $V_{l}$, i.e. $u$ and $v$, and the solution is projected through the intermediate levels $G_{l-1}, G_{l-2}, \cdots$, until $G_{0}$. For each decomposed $s V \in V_{l+1}$, its predecessor-vertices $\{u, v\} \in V_{l}$ are assigned to the same community as their successor $s V \in G_{l+1}$. Figure 54 illustrates this process: super-vertex $s V=\{4,5\}$ is expanded to its original vertices 4 and 5 , which are assigned to the same community as $s V$.

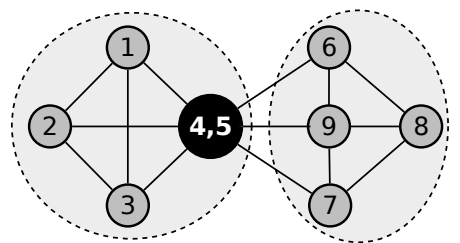

(a)

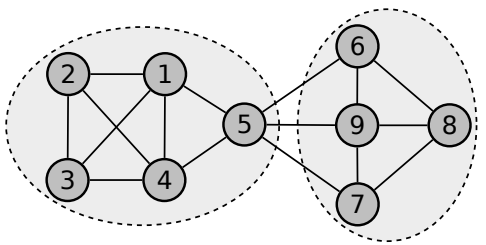

(b)

Figure 54 - Super-vertex $s V$ formed by vertices 4 and 5 is expanded and each single vertex is assigned to the same community as $s V$.

Source: Elaborated by the author.

\subsection{Community detection based on multilevel method by using one-mode projection}

This section introduces a multilevel community detection method that handles bipartite networks. Standard multilevel methods do not distinguish between vertex types, whereas the 
layers in bipartite networks usually represent different entities and should be handled independently. Therefore, usual matching algorithms, such as RM, HEM and GHEM are not directly applicable. Nonetheless, they can be applied to a one-mode projection of $G$ (i.e., $G^{1}$ and $G^{2}$ ), since the projection produces two one-mode networks, in which vertices are all of the same type. Therefore, one-mode projection methods enable applying any classical coarsening algorithm to bipartite networks after a transformation process. This concept was considered to introduce a multilevel community detection method applicable to bipartite networks.

Algorithm 13 summarizes the implementation of the proposed one-mode projectionbased multilevel method for community detection (OPM). It comprises the phases of coarsening (lines 1-6), community detection (line 9) and uncoarsening (lines 10-12). The inputs are the initial bipartite network $G=(V, E, \sigma, \omega)$, and for each layer a maximal number of levels $L$ and a reduction factor $\rho$.

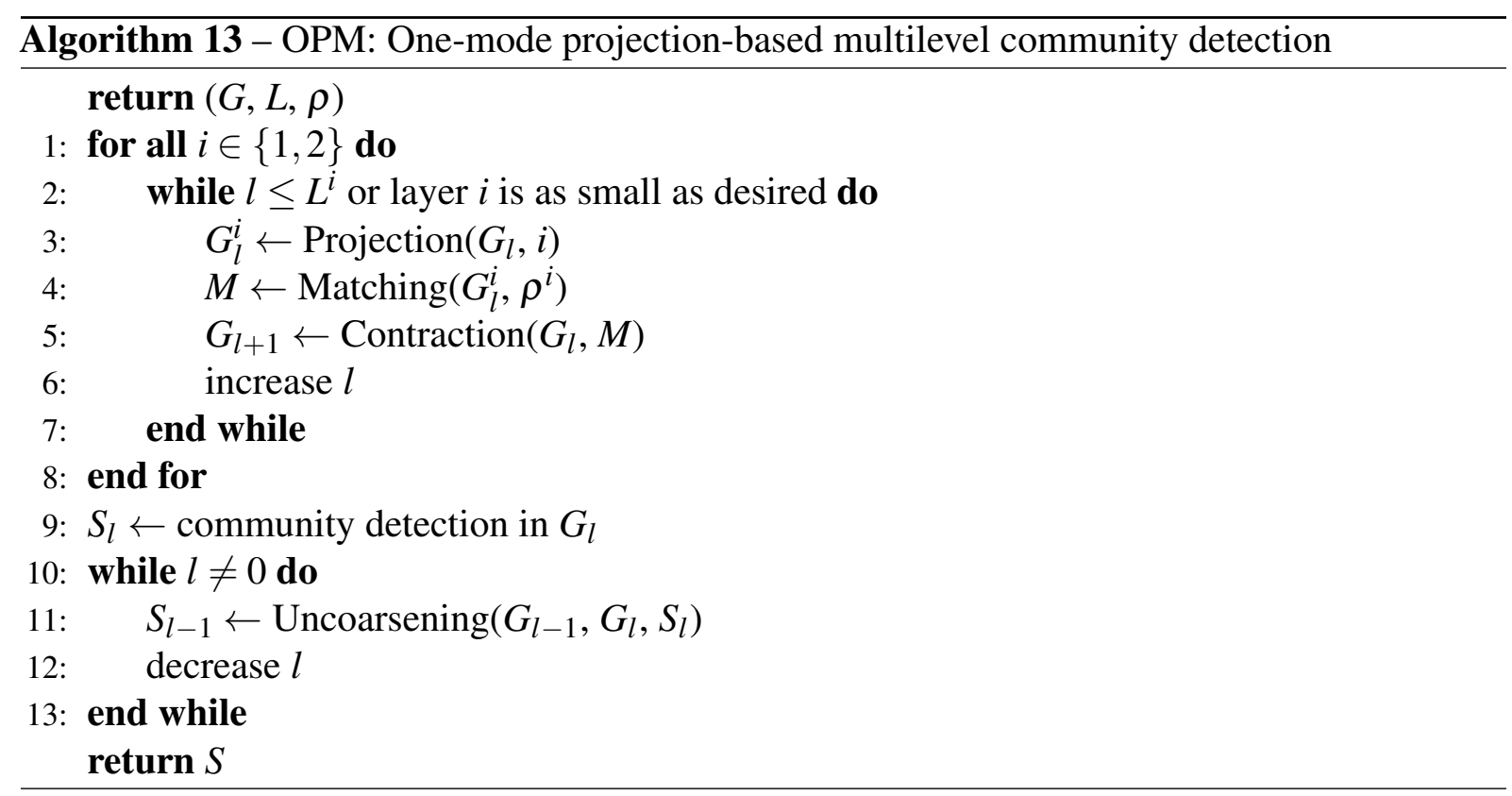

The bipartite network initially undergoes a one-mode projection transformation, being split into two unipartite networks $G^{1}$ and $G^{2}$ (line 3). A coarsening process is then applied to each network $G^{1}$ and $G^{2}$ separately (lines 4-5), level by level, until each one has been reduced by the desired factor. The process comprises a matching algorithm (line 4) and a contraction algorithm (line 5) is applied in $G_{l}$ from $M$. In this study, we considered the aforementioned matching algorithms RM, HEM and GHEM (KARYPIS; KUMAR, 1998a).

An initial community structure $S_{L}$ (the initial solution) is then obtained on the coarsest bipartite network $G_{L}$, at level $l=L$ (line 9). As $G_{L}$ and $S_{L}$ are, respectively, the input and output at this stage $\left(S_{L}\right.$ representing the community structure found in network $\left.G_{L}\right)$, different algorithms for community detection can be considered to compute this initial solution. Depending on the settings of the coarsening phase, the coarsest bipartite network can be very small, so that computationally expensive algorithms can be considered as possible alternatives (see Section 
4.4).

Finally, in the subsequent uncoarsening phase (lines 10-12), solution $S_{L}$ (as $l=L$ ) is projected back to $G_{0}$ through the intermediate levels $G_{l-1}, G_{l-2}, \ldots, G_{1}, G_{0}$ (line 11). For the specific case of a community detection problem, $S_{L}$ is described as a partitioning of the vertex set into non-empty partitions $P_{k}$ with $\cup P_{k}=S_{L}, P_{k} \subseteq V_{L}$. Following previous guidelines proposed for the uncoarsening process (KARYPIS; KUMAR, 1998a), a solution $S_{l}$ is constructed from $S_{l+1}$ simply by assigning vertices $\{u, v\} \in V_{l}$ to the same community of their successor super-vertex $s V \in V_{l+1}$.

\subsection{Experimental Results and Analysis}

We implemented $O P M$ and investigated whether it can be employed to scale a costly community detection algorithm, whilst preserving solution quality. We considered the recent $L P A w b+$ algorithm introduced by Beckett (BECKETT, 2016) ${ }^{1} . L P A w b+$ maximizes Barber's modularity through label propagation in weighted bipartite networks. Beckett has shown it has competitive performance compared with state-of-the-art methods. However, it is computationally expensive and thus only feasible on small-scale networks.

The $O P M$ framework was thus validated by taking Beckett's $L P A w b+$ as the target algorithm. Therefore our implementation of $O P M$ (Algorithm 13) performs the coarsening, then runs $L P A w b+$ to find the community structure in the coarsest network, and finally projects the solution to obtain the community structure in the original network.

We investigated three alternative instantiations of $O P M$ that adopt the matching algorithms GHEM, HEM and RM, hereafter referred to as $O P M_{\text {ghem }}, O P M_{h e m}$ and $O P M_{r m}$, respectively. Each instantiation was executed with parameters $\rho=0.5$ and $L=[1,2,3]$ in a set of 15 synthetic weighted bipartite networks, identified as R1-R15. Synthetic networks were obtained with the community model described in (BECKETT, 2016), which creates networks with unbalanced and randomly positioned community structures. Networks of sizes $n=\left|V^{1}\right|+\left|V^{2}\right|$ were generated within the range $[1,000 ; 15,000]$ at increments of 1,000 and the number of communities was set to $0.01 \mathrm{n}$. Edge weights were randomly assigned from a skewed negative binomial distribution and noise was introduced in the connection patterns by reconnecting a percentage of the edges between and within communities.

Performance was measured by means of the normalized mutual information (NMI), which compares a solution found by a particular algorithm with a reference solution (LABATUT, 2013), and we also computed execution times. The experiments were executed in a Linux machine with 8 core processor $3.7 \mathrm{GHz}$ CPU and $64 \mathrm{~GB}$ main memory. The framework ${ }^{2}$ was

$1<$ https://github.com/sjbeckett/weighted-modularity-LPAwbPLUS.>

$2<$ https://github.com/alanvalejo/opm> 
implemented in Python with the igraph library ${ }^{3}$. We report average values obtained from 30 executions for algorithm instances that rely on random choices for matching $\left(O P M_{h e m}\right.$ and $\left.O P M_{r m}\right)$.

Table 10 shows the accuracy values as measured by NMI on the 15 synthetic networks. The highest values are shown in bold and accuracies equivalent or superior to the baseline solution are highlighted with a gray background.

\begin{tabular}{|l|r|r|r|r|r|r|r|r|r|r|r|r|r|r|r|r|}
\hline \multicolumn{10}{|c|}{ Algorithm } & \multicolumn{10}{|c|}{ Dataset } \\
\hline Name & Levels $[L]$ & $\mathrm{R} 1$ & $\mathrm{R} 2$ & $\mathrm{R} 3$ & $\mathrm{R} 4$ & $\mathrm{R} 5$ & $\mathrm{R} 6$ & $\mathrm{R} 7$ & $\mathrm{R} 8$ & $\mathrm{R} 9$ & $\mathrm{R} 10$ & $\mathrm{R} 11$ & $\mathrm{R} 12$ & $\mathrm{R} 13$ & $\mathrm{R} 14$ & $\mathrm{R} 15$ \\
\hline \hline LPAwb+ & 0 & 0.918 & 0.926 & 0.983 & 0.972 & 0.964 & 0.990 & 0.984 & $\mathbf{0 . 9 9 9}$ & $\mathbf{0 . 9 9 9}$ & 0.985 & 0.989 & $\mathbf{0 . 9 9 6}$ & $\mathbf{0 . 9 9 5}$ & 0.987 & 0.992 \\
\hline \hline OPM ghem & 1 & $\mathbf{0 . 9 9 2}$ & 0.982 & $\mathbf{0 . 9 8 5}$ & $\mathbf{0 . 9 9 0}$ & 0.991 & $\mathbf{0 . 9 9 3}$ & $\mathbf{0 . 9 9 0}$ & 0.992 & 0.991 & $\mathbf{0 . 9 9 5}$ & $\mathbf{0 . 9 9 1}$ & 0.991 & 0.994 & 0.990 & $\mathbf{0 . 9 9 5}$ \\
OPM $_{\text {ghem }}$ & 2 & 0.981 & $\mathbf{0 . 9 8 7}$ & 0.980 & 0.986 & 0.991 & 0.989 & 0.988 & 0.991 & 0.988 & 0.989 & $\mathbf{0 . 9 9 1}$ & 0.990 & 0.991 & 0.989 & 0.993 \\
OPM $_{\text {ghem }}$ & 3 & 0.875 & 0.952 & 0.960 & 0.964 & 0.963 & 0.971 & 0.972 & 0.975 & 0.973 & 0.975 & 0.973 & 0.974 & 0.976 & 0.976 & 0.976 \\
\hline \hline OPM $_{\text {hem }}$ & 1 & 0.991 & $\mathbf{0 . 9 8 7}$ & $\mathbf{0 . 9 8 5}$ & 0.984 & $\mathbf{0 . 9 9 1}$ & 0.990 & 0.985 & 0.992 & 0.991 & $\mathbf{0 . 9 9 5}$ & 0.990 & 0.991 & 0.992 & $\mathbf{0 . 9 9 1}$ & 0.993 \\
OPM $_{\text {hem }}$ & 2 & 0.973 & 0.985 & 0.981 & 0.982 & 0.989 & 0.987 & 0.989 & 0.989 & 0.988 & 0.989 & 0.989 & 0.988 & 0.989 & 0.990 & 0.991 \\
OPM $_{\text {hem }}$ & 3 & 0.873 & 0.952 & 0.960 & 0.966 & 0.963 & 0.971 & 0.972 & 0.975 & 0.972 & 0.975 & 0.973 & 0.974 & 0.975 & 0.976 & 0.976 \\
\hline \hline OPM $_{r m}$ & 1 & 0.312 & 0.358 & 0.409 & 0.412 & 0.407 & 0.414 & 0.442 & 0.462 & 0.448 & 0.462 & 0.468 & 0.483 & 0.483 & 0.502 & 0.498 \\
OPM $_{r m}$ & 2 & 0.146 & 0.169 & 0.147 & 0.135 & 0.158 & 0.171 & 0.157 & 0.162 & 0.150 & 0.150 & 0.150 & 0.161 & 0.148 & 0.152 & 0.161 \\
OPM $_{r m}$ & 3 & 0.100 & 0.119 & 0.119 & 0.098 & 0.105 & 0.105 & 0.090 & 0.079 & 0.082 & 0.069 & 0.078 & 0.099 & 0.084 & 0.082 & 0.072 \\
\hline
\end{tabular}

Table 10 - NMI accuracy values of the three $O P M$ instantiations and baseline $L P A w b+$ in 15 synthetic networks (averages over 30 executions for $O P M_{h e m}$ and $O P M_{r m}, O P M_{g h e m}$ is deterministic). We highlight the highest accuracy values, shown in bold, and values equivalent or superior to the baseline $(L P A w b+)$, shown in shaded cells.

Source: Research data.

The best performances were achieved by $O P M_{\text {ghem }}$ with one level of coarsening $(L=1)$ on 11 out of the 15 networks. The baseline community detection algorithm $L P A w b+$ yielded the best performance in three out of the 15 networks, whereas the worst results were obtained with $O P M_{r m}$ with $L=3$.

Indeed, the random strategy $R M$ yields extremely poor accuracies, which renders its application unfeasible in real contexts. As opposite, the accuracy values attained by HEM and the strategy GHEM are similar or superior to those of $L P A w b+$. Not surprisingly, limited coarsening levels (mainly $L=1$ ) yielded higher accuracy values, and accuracy decreases as the coarsening level increases $(L=3)$. For $L=3$ the extensive collapsing of vertices tends to blur the boundaries between adjacent communities. The effect of parameter $L$ depends on network size, i.e., differences in algorithm accuracy are likely to decrease as network sizes increase, which suggests it may be possible to adopt higher values of $L$ on larger networks without such an expressive loss in solution quality.

A Nemenyi post-hoc test (DEMSAR, 2006) was applied to the results shown in Table

$3<$ http://igraph.org/python/> 
10 to detect statistical differences in the performances of the different instances. The results are shown in Figure 55 for $55 \mathrm{a} L=1,55 \mathrm{~b} L=2$ and $55 \mathrm{c} L=3$. The critical difference (CD) is indicated at the top of each diagram and the methods' average ranks are placed on the horizontal axes (better ranked on the left). A black line connects algorithms if no significant difference has been detected between them.

According to the Nemenyi statistics, the critical value for comparing the mean-ranking of two different algorithms at 95 percentile is 1.21 for all diagrams. Mean-rankings differences above this value are significative. When $L=1$ (Figure 55a), OPM ghem was ranked best, followed by $O P M_{h e m}, L P A w b+$ and, finally, $O P M_{r m}$. Furthermore, no statistically significant difference was observed between $O P M_{\text {ghem }}, O P M_{h e m}$ and $L P A w b+$. For $L=2$ (Figure 55b), $O P M_{\text {ghem }}$, $O P M_{h e m}$ and $L P A w b+$ were ranked first and no statistically significant difference was observed between them. Finally, for $L=3$ (Figure 55c) $L P A w b+$ was ranked first with a statistically significant difference between $O P M_{g h e m}, O P M_{h e m}$ and $O P M_{r m}$.

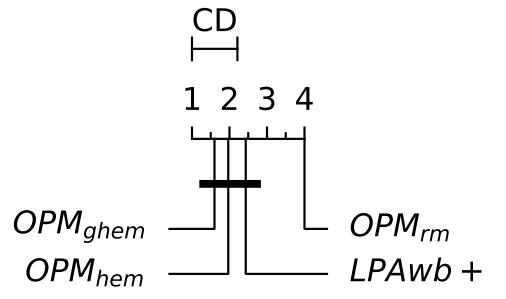

(a) $L=1$

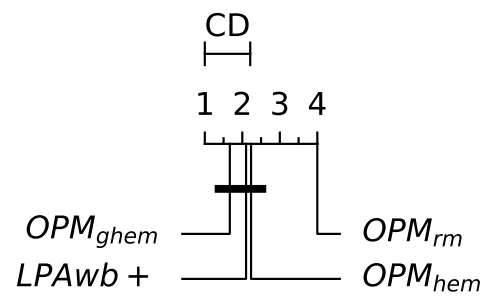

(b) $L=2$

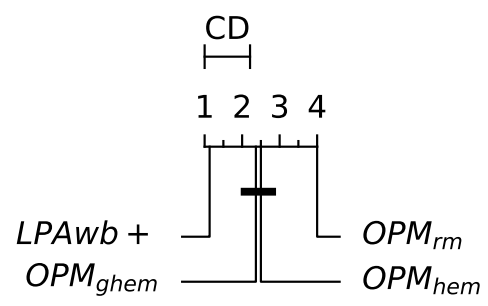

(c) $L=3$

Figure 55 - Nemenyi post-hoc test applied to the results from $L P A w b+$ and two $O P M$ variants, for the three settings of parameter $L$.

Source: Elaborated by the author.

Figure 56 depicts the averages and standard deviations of the accuracy values, whereas Figure 57 shows their distribution dispersion and outliers, in both cases for the three alternative settings of parameter $L$. As results from $O P M_{r m}$ were very poor, in order to improve legibility we suppress it from the figures and show only bar and box plots for $O P M_{g h e m}, O P M_{h e m}$ and $L P A w b+$.

The bar plots in Figure 56 reveal superior performance and stability of both $O P M$ instances when $L=1$, confirmed by their higher average accuracies and narrower standard deviations. For $L=3, L P A w b+$ yielded better results than either $O P M$ instance, a consequence of the extensive network reduction. The box plots in Figure 57a reveal that for $L=1$ all $O P M$ 
instances yielded accuracy values with higher averages and narrower distributions than $L P A w b+$.

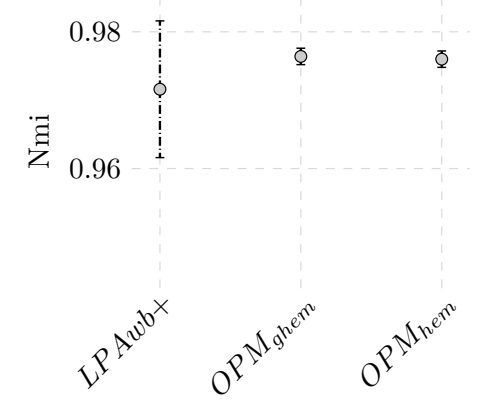

(a) $L=1$

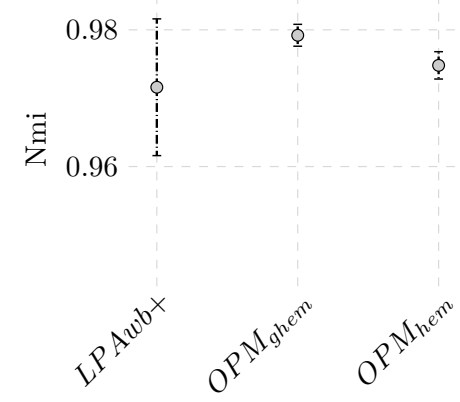

(b) $L=2$

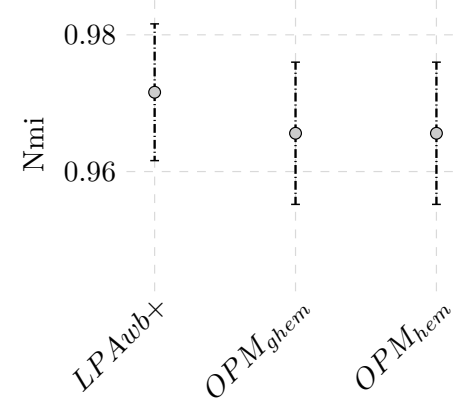

(c) $L=3$

Figure 56 - Averages and standard deviations of the NMI accuracy values obtained with $L P A w b+$ and two $O P M$ instances in three alternative settings of parameter $L$ (number of levels). (a) $L=1$, (b) $L=2$ and (c) $L=3$.

Source: Elaborated by the author.

We conclude $O P M$ instances yielded, in general, higher accuracy and improved stability in comparison to standard $L P A w b+$. In summary, the experimental evidence regarding solution quality (averages, standard deviations and dispersion of the accuracy values) suggests the multilevel framework stabilizes and improves the performance of the algorithm.

The scalability that can be attained with $O P M$ was assessed considering the performance of its three instantiations on each individual network. Table 11 shows the absolute execution times (in seconds) of each instance in each network. For $O P M_{h e m}$ and $O P M_{r m}$ values refer to average times relative to 30 executions.

The longest execution time of algorithm $L P A w b+$ was 302,442 seconds (time to process the largest network, R15) and the shortest was 14 seconds (time to process the smallest one, R1). The most expensive multilevel instance $O P M_{\text {ghem }}(L=1)$ consumed 76,439 in R15 and 10 seconds in R1; hence, $O P M_{\text {ghem }}$ run 3.9 to 1.4 times faster than $L A P w b+$, relative to their maximum and minimum execution times, respectively. $O P M_{\text {hem }}(L=1)$ consumed 36,439 seconds in R15 and 3 seconds in R1. Therefore, $O P M_{h e m}$ run 8.3 to 4.6 times faster than $L A P w b+$. The maximum and minimum times of the least expensive instance $O P M_{r m}(L=3)$ were 903 seconds and 3 seconds, respectively. Therefore, $O P M_{r m}$ run 335 to 4.6 times faster than $L A P w b+$. 


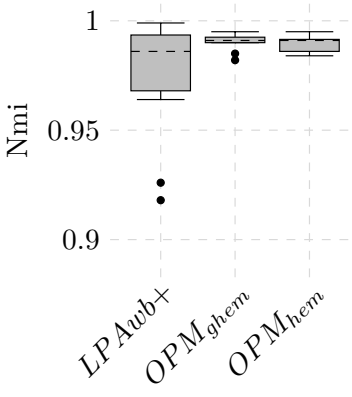

(a) $L=1$

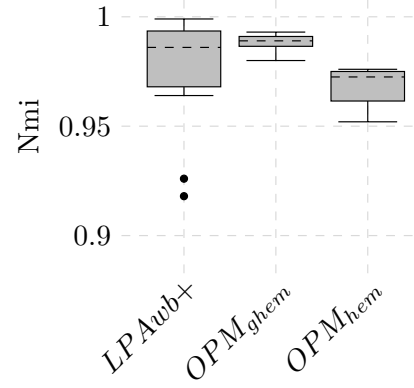

(b) $L=2$

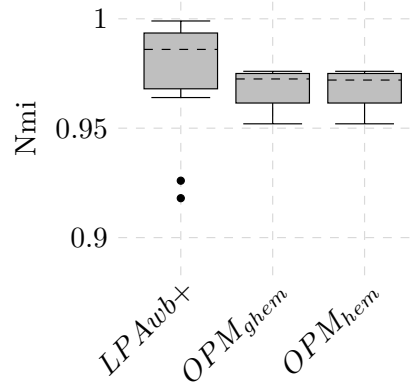

(c) $L=3$

Figure 57 - Shape distribution, variability, and averages of the accuracy values yielded by $L P A w b+$ and the two instances of $O P M$ considering three settings of parameter $L$. (a) $L=1$, (b) $L=2$ and (c) $L=3$.

Source: Elaborated by the author.

The total time spent running the experiments was 1,558,583 seconds, or nearly 433 hours. In the best case, algorithm implementation $O P M_{r m}$ with $L=3$ reduced the execution time from 851,876 seconds (nearly 236 hours), required by the standard $L P A w b+$, to 5,515 seconds (1.5 hours), which implies the standard implementation of $L P A w b+$ was nearly 154 times slower than this particular implementation of $O P M$.

\begin{tabular}{|c|c|c|c|c|c|c|c|c|c|c|c|c|c|c|c|c|c|}
\hline \multicolumn{2}{|c|}{ Algorithm } & \multicolumn{15}{|c|}{ Dataset } & \multirow{2}{*}{ sum } \\
\hline Name & Levels $[L]$ & R1 & $\mathrm{R} 2$ & R3 & $\mathrm{R} 4$ & $\mathrm{R} 5$ & R6 & R7 & $\mathrm{R} 8$ & R9 & R10 & R11 & $\mathrm{R} 12$ & R13 & R14 & R15 & \\
\hline$L P A w b+$ & 0 & 14 & 96 & 308 & 904 & 2,782 & 2,800 & 7,146 & 5,925 & 9,197 & 56,119 & 66,729 & 75,990 & 97,392 & 224,032 & 302,442 & 851,876 \\
\hline OPM ghem & 1 & 10 & 21 & 48 & 327 & 516 & 540 & 1,573 & 2,830 & 4,289 & 8,922 & 11,169 & 18,214 & 28,263 & 41,945 & 76,439 & 320,012 \\
\hline OPM ghem & 2 & 8 & 16 & 21 & 201 & 302 & 169 & 661 & 723 & 1,984 & 3,199 & 8,112 & 10,219 & 16,853 & 19,825 & 31,591 & 82,970 \\
\hline$O P M_{\text {ghem }}$ & 3 & 3 & 10 & 13 & 123 & 180 & 142 & 291 & 329 & 528 & 1,810 & 2,098 & 3,991 & 4,517 & 5,767 & 10,892 & 41,859 \\
\hline$O P M_{h e m}$ & 1 & 3 & 14 & 43 & 127 & 326 & 540 & 973 & 1,933 & 2,989 & 6,983 & 9,628 & 13,695 & 18,473 & 22,875 & 36,439 & 115,041 \\
\hline$O P M_{h e m}$ & 2 & 2 & 6 & 21 & 51 & 106 & 139 & 296 & 396 & 854 & 2,215 & 3,112 & 3,776 & 5,442 & 6,826 & 10,538 & 33,780 \\
\hline$O P M_{h e m}$ & 3 & 1 & 6 & 13 & 33 & 60 & 55 & 80 & 128 & 264 & 818 & 1,604 & 1,889 & 2,351 & 2,863 & 3,769 & 13,934 \\
\hline$O P M_{r m}$ & 1 & 8 & 57 & 124 & 220 & 419 & 476 & 780 & 1,484 & 1,991 & 5,660 & 9,619 & 13,127 & 11,291 & 17,691 & 19,202 & 82,149 \\
\hline$O P M_{r m}$ & 2 & 4 & 25 & 40 & 58 & 103 & 150 & 146 & 179 & 345 & 1,005 & 1,626 & 1,873 & 1,902 & 1,950 & 2,041 & 11,447 \\
\hline$O P M_{r m}$ & 3 & 3 & 16 & 28 & 33 & 49 & 67 & 64 & 83 & 194 & 712 & 754 & 849 & 870 & 890 & 903 & 5,515 \\
\hline \multicolumn{2}{|c|}{ sum } & 56 & 267 & 659 & 2,077 & 4,843 & 5,078 & 12,010 & 14,010 & 22,635 & 87,443 & 114,451 & 143,623 & \begin{tabular}{|l|}
187,354 \\
\end{tabular} & 344,664 & 494,256 & $1,558,583$ \\
\hline
\end{tabular}

Table 11 - Absolute runtime (seconds) of $L P A w b+$ and four OPM instances on each network.

Source: Research data.

Executing algorithm $L P A w b+$ consumed over $54.6 \%$ of the time spent in the experiments, 
whereas roughly $20.5 \%$ of the time was spent running the most expensive instance $O P M_{\text {ghem }}$ $(L=1)$ and $0.35 \%$ of the time was spent running the least expensive instance $O P M_{r m}(L=3)$.

From this empirical investigation we conclude the proposed $O P M$ approach can scale the standard $L P A w b+$ whilst yielding more accurate and stable results. Although solution quality degrades as the network is progressively coarsened, runtime drops drastically at each additional coarsening level; hence, a successful solution to the problem requires establishing a suitable trade-off between accuracy and execution time, i.e., identifying a suitable network reduction factor.

\subsection{Conclusions}

In this paper we introduced an approach that enables using the multilevel paradigm to scale an expensive community detection algorithm to large-scale bipartite networks. While previous multilevel methods consider only unipartite networks, our one-mode projection-based multilevel method enables handling bipartite networks with standard coarsening algorithms.

Tests on a large suite of synthetic networks have shown that this solution yields results with accuracy comparable to that of the original method and demands considerably shorter execution times. Our tests compared the outcome of the solution implemented with three popular matching strategies for coarsening, namely GHEM, HEM and RM. RM yielded expressive speedups or even improved the asymptotic convergence, but with poor results regarding accuracy, which prevents its practical application. $H E M$ achieved rather good approximation to the standard method in terms of accuracy and acceptable speedups, e.g., execution times over 4.6 times shorter as compared to the standard method. Finally, although GHEM is more costly than RM and HEM, it proved more robust in terms of solution quality, in the test cases considered.

Some issues deserve further attention, such as investigating alternative refinement strategies for the uncoarsening phase and parallel or distributed paradigms to further increase scalability. Another relevant issue is to explore how the choice of $\rho$, the reduction factor parameter, impacts accuracy and speedups on different application scenarios.

Our implementation of the one-mode projection multilevel solution can be downloaded at $<$ https://github.com/alanvalejo/opm>. 

CHAPTER

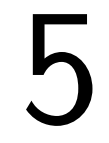

\section{MULTILEVEL APPROACH FOR COMBINATORIAL OPTIMIZATION IN BIPARTITE NETWORKS}

Multilevel methods aim at reducing the cost of a target algorithm over a given network by applying it to a coarsened (or reduced) version of the original network. They have been successfully employed in a variety of problems, most notably community detection. However, current solutions are not directly applicable to bipartite networks and the literature lacks studies that illustrate their application for solving problems in such networks using the multilevel method. This article addresses this gap and introduces a multilevel method for bipartite networks and the implementation of a general multilevel framework including novel algorithms for coarsening and uncorsening, applicable to a variety of problems. We analyze how the proposed multilevel method affects the topological features of bipartite networks and show that a controlled coarsening strategy can preserve properties such as degree and clustering coefficient centralities. The applicability of the general framework is illustrated in two optimization problems, one for solving the Barber's modularity for community detection and the second for dimensionality reduction in text classification. We show that the solutions thus obtained are statistically equivalent, regarding the accuracy, to those of conventional approaches, while requiring considerably lower execution times.

\subsection{Introduction}

Bipartite networks comprise a particular class of network models in which the set of vertices is split into two disjoint subsets, with edges connecting vertices placed in different sets. Also known as two-layer networks, they provide a powerful representation of relationships in many real-world systems, including document-word (ROSSI; LOPES; REZENDE, 2016), protein-ligand (JEONG et al., 2000), actor-movie (WATTS; STROGATZ, 1998), georeferenced 
user-location (WANG et al., 2014b) and paper co-authorship or citation networks (NEWMAN, 2001c). Bipartite network models have been widely employed in hard combinatorial optimization problems that require finding a minimum (or maximum) cost, wherein the number of possible states is finite and usually exponential. Many such problems, e.g., biclique, matching, vertex cover, community structure, traveling salesman and network coloring (ASRATIAN; DENLEY; HäGGKVIST, 1998) have proven to be $N P$-complete or $N P$-hard.

Multilevel techniques are being investigated as a global strategy to handle decisionmaking and optimization problems in a variety of application domains. We refer the reader interested in applications to management problems to the recent literature on the topic (ZHANG; AHN, 2015; HAN et al., 2015; LU et al., 2016). In this paper, we investigate the multilevel method to handle computationally expensive optimization problems in bipartite networks. In this context, the approach consists of iteratively coarsening an original network into a hierarchy of smaller sized approximations. A initial solution is obtained in the coarsest network and successively projected back and refined over the inverse sequence of coarsened networks, until the original one. Previous studies demonstrated the strategy enables running computationally expensive algorithms on large networks with no significant loss in solution quality (BRANDT, 1988; KARYPIS; KUMAR, 1995a; TENG, 1999; NOACK; ROTTA, 2009; VALEJO et al., 2014a; VALEJO; VALVERDE-REBAZA; LOPES, 2014a; LASALLE; KARYPIS, 2015). Sciences (WALSHAW, 2004) and Walshaw (WALSHAW, 2008a) argued over the relevance and feasibility of the multilevel method for solving combinatorial optimization problems. Empirical evidence has been shown by Walshaw (WALSHAW, 2008a) that the coarsening process filters the solution space by gradually removing irrelevant high-cost solutions and drastically reducing the search space, and hence, optimization convergence times.

Multilevel algorithms have been applied to many classic network problems, including network coloring (WALSHAW, 2001a), traveling salesman (WALSHAW; EVERETT, 2002), network drawing (WALSHAW, 2001a), network partitioning (BICHOT, 2014) and computation of centrality measures (CHERNOSKUTOV; INEICHEN; BEKAS, 2015). However, current multilevel methods are not directly applicable to bipartite networks and, to the best of our knowledge, the multilevel method has not been considered in this context.

We address this gap and introduce a novel multilevel method applicable to bipartite networks. Furthermore, we describe an implementation of this approach as a general-purpose multilevel framework that incorporates two novel efficient matching algorithms, as well as novel contraction and uncoarsening algorithms.

To illustrate its potential, we employed the framework to handle two distinct problems defined in bipartite networks, namely community detection and dimensionality reduction. In the community detection problem, tests on a large set of synthetic networks demonstrated that, combined with a proper local search strategy, it yields good speedups and preserves solution quality. When employed to perform dimensionality reduction in text classification it 
yielded encouraging results in terms of both runtime and accuracy as compared with a standard dimensionality reduction technique.

The remainder of the paper is organized as follows: Section 5.2 reviews some basic concepts on networks and provides an overview of the standard multilevel method. Section 5.3 discusses previous work and application of multilevel methods on combinatorial optimization problems. Section 5.4 introduces a multilevel formulation for bipartite networks and its implementation. Section 5.5 reports our empirical results, which include (i) an analysis of how the multilevel representation impacts the topological features of a real bipartite network; (ii) an empirical study of instantiating the framework to solve the community detection problem on a large synthetic test suite; and (iii) an empirical study of its application to dimensionality reduction in text classification. Section 5.6 briefly discusses how the general framework can be tuned for application in other types of problems. Finally, Section 5.7 summarizes our findings and discusses future work.

\subsection{Background}

\subsubsection{Basic concepts}

Let $G=(V, E, \sigma, \omega)$ be an undirected weighted network, where $V=\{1, \ldots, n\}$ denotes the set of vertices and $E \subseteq V \times V$ denotes the set of edges, such that $(v, u)=\{(u, v)=(v, u) \mid$ $u, v \in V\}$. Let $n=|V|$ be the total number of vertices and $m=|E|$ be the total number of edges, where operator "|.|" stands for the cardinality of a set. The weight of an edge $(u, v)$ is represented by $\omega(u, v)$ with $\omega: V \times V \rightarrow \mathbb{R}^{*}$ and the weight of a vertex $v$ is represented by $\sigma(v)$ with $\sigma: V \rightarrow \mathbb{R}^{*}$.

A network $G=(V, E, \sigma, \omega)$ is bipartite (two-layer network) if $V$ is partitioned into two sets $V^{1}$ and $V^{2}$, such that $V^{1} \cap V^{2}=\emptyset$ and $E \subseteq V^{1} \times V^{2}$. Hereafter, each vertex subset is called a layer. A bipartite network thus has two layers so that vertices in the same layer are not connected.

The degree of a vertex $v \in V$, denoted $\kappa_{v}$, is given by the total weight of its adjacent edges, i.e. $\kappa_{v}=\sum_{u \in V} w(v, u)$. The $h$-hop neighborhood of $v$, denoted $\Gamma_{h}(v)$, is formally defined as the vertices in set $\Gamma_{h}(v)=\{u \mid$ there is a path of length $h$ between $v$ and $u\}$. Thus, the 1-hop neighborhood of $v, \Gamma_{1}(v)$, is the set of vertices adjacent to $v$; the 2-hop neighborhood, $\Gamma_{2}(v)$, is the set of vertices 2-hops away from $v$, and so forth.

A similarity score $S(u, v)$ can be computed from a pair of vertices $u$ and $v$. A fundamental structural similarity function between a pair of vertices is given by the number of common neighbors, defined as $S_{c n}(u, v)=|\Lambda(u, v)|, \Lambda(u, v)=\left\{\Gamma_{1}(u) \cap \Gamma_{1}(v)\right\}$. Alternatively, a weighted common neighbors similarity function can be defined by equation 5.1, where the term $\log (1+$ 
$s(z))$ is used to prevent negative scores (LÜ; ZHOU, 2010).

$$
S_{w c n}(u, v)=\sum_{z \in \Lambda(u, v)} \frac{\omega(u, z)+\omega(v, z)}{\log (1+s(z))} \quad s(u)=\sum_{z \in \Gamma_{1}(u)} \omega(u, z)
$$

The local clustering coefficient of a vertex is given by the probability of its neighbors being connected (WATTS; STROGATZ, 1998; NEWMAN, 2003). This statistics is closely related to transitivity, which measures the relative frequency of triangles in the vertex neighborhood. The clustering coefficient is defined by equation 5.2:

$$
c c(v)=\frac{2 \operatorname{tr}(v)}{\left|\Gamma_{1}(v)\right|\left(\left|\Gamma_{1}(v)\right|-1\right)},
$$

where $\operatorname{tr}(u)$ denotes the number of edges $(v, z) \in E$, such that $v, z \in \Gamma_{1}(u)$ and $c c$ relies on the enumeration of the triangles in the network. However, as triangles do not occur in bipartite networks this definition is not valid. An equivalent metrics for bipartite graphs was introduced by Latapy et al. (LATAPY; MAGNIEN; VECCHIO, 2008), defined in equation 5.3, which captures the overlap between vertex neighborhoods in the same layer.

$$
c c_{b}(v, u)=\frac{\left|\Gamma_{1}(u) \cap \Gamma_{1}(v)\right|}{\left|\Gamma_{1}(u) \cup \Gamma_{1}(v)\right|} \quad c c_{b}(v)=\frac{\sum_{v \in \Gamma_{2}(u)} c c_{b}(v, u)}{\left|\Gamma_{2}(u)\right|}
$$

\subsubsection{General-purpose multilevel method}

A multilevel method is formally defined as a meta-heuristics that combines different heuristics to guide, modify and possibly fix a solution obtained from a target algorithm (or operations of the subordinate heuristics, local search or global search) and refines this solution over multiple iterations. It operates in three phases, namely coarsening, solution finding and uncoarsening. In the coarsening phase, the network size is successively reduced to obtain coarser network representations; in the solution finding phase a initial solution is obtained applying the target algorithm in the coarsest representation; in the uncoarsening phase, the initial solution is successively projected back to the intermediate networks and refined, until obtaining the final solution.

Figure 58 illustrates such a process, considering an initial network $G_{0}$ (in which the original problem instance is defined), where $G_{L}$ denotes the coarsest network obtained after $L$ coarsening steps (levels), $S_{L}$ denotes the initial solution obtained in $G_{L}$, and $S_{0}$ denotes the final refined solution obtained in $G_{0}$.

\section{Coarsening}

The coarsening phase constructs a hierarchy of coarsened networks $G_{l}$ from the initial network $G_{0}$, yielding intermediate network approximations on multiple levels-of-detail. 


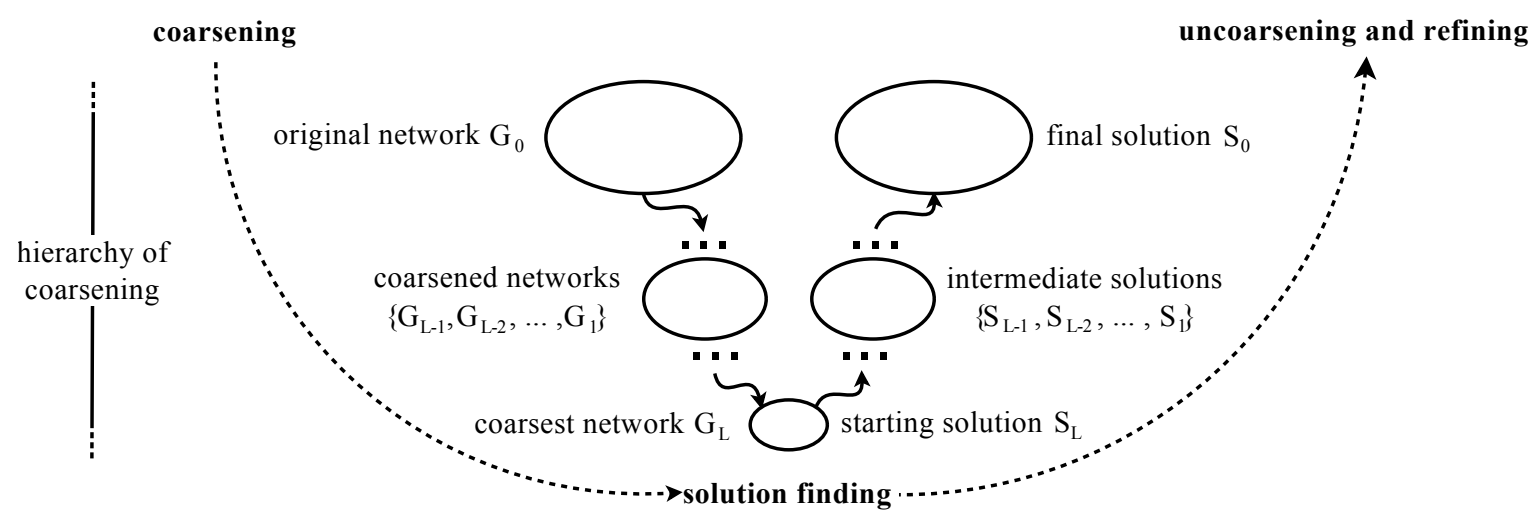

Figure 58 - Phases of a multilevel method: coarsening, solution finding and uncoarsening.

Source: Elaborated by the author.

The process requires two algorithms, namely matching, which defines which vertices will be collapsed, and contraction, which builds the reduced representation, given the matching. Let $G_{l}=\left(V_{l}, E_{l}, \sigma_{l}, \omega_{l}\right)$ be the network model coarsened at level $l$, with $\left|V_{0}\right|>\left|V_{1}\right|>\ldots>\left|V_{L}\right|$.

Coarsening starts with the matching step. According to some given restriction, in general, pairs of vertices are selected for matching, producing a set of unordered pairs called vertex matching, independent edge set or simply matching. Formally, a matching $M$ consists of a set of pairwise non-adjacent edges. Heavy-edge matching is a popular algorithm for this purpose, which attempts to find a matching of maximal weight (KARYPIS; KUMAR, 1998a).

At any level, the coarsening of a network must preserve its topological features, implying that vertex and edge-weights of the reduced network must reflect the connectivity of its predecessors. This will be guaranteed by a proper choice of matching strategy, which is a key component of effective multilevel method. A matching strategy inadequate to support the solution finding phase will impair the quality of the solution derived by a multilevel algorithm and its performance.

Once the matching is defined, a contraction algorithm constructs the coarsened network, by collapsing matched vertex pairs into a single super-vertex $(s V)$. A successor-network $G_{l+1}$ will inherit the non-collapsed vertices from its predecessor. In order for $G_{i+1}$ to be a good proxy to its predecessor, given a super-vertex $s V=\{v, u\} \in V_{i+1}$ its weight $\sigma(s V)$ is computed as the sum of weights $\sigma(v)$ and $\sigma(u),\{u, v\} \in V_{i}$. Furthermore, the edges incident to vertices $\{u, v\} \in V_{i}$ are collapsed to obtain the so-called super-edges incident to $s V$.

\section{Solution finding}

This phase employs the target algorithm to solve the problem on the coarsest network $G_{l}$. Let $S$ be the set of all possible solutions in the coarsest problem instance. Given an objective (cost) function $f: S \rightarrow \mathbb{R}$ (or $\mathbb{N}$ ) that assigns a cost to each solution in $S$, the aim is to find a state $s \in S$ with minimum (or maximum) cost. For instance, in the traveling salesman problem 
$f(s)$ expresses the length of tour $s$, whereas in a network community detection problem it denotes some measure of community quality. Since the coarsest network is possibly very small, it becomes feasible to employ computationally expensive target algorithms to find a initial solution (KARYPIS; KUMAR, 1995a).

\section{Uncoarsening and refinement}

The uncoarsening (also known as solution projection) phase successively transfers the solution available at a current level to the upper level in the hierarchy, i.e., the solution obtained in the coarsest network $G_{l}$ is successively projected through intermediate networks $G_{L-1}, G_{L-2}, \ldots, G_{1}$ up to the original network $G_{0}$.

Solution $S_{l}$ is constructed from $S_{l+1}$ simply by assigning vertices $\{u, v\} \in V_{l}$ to the same set of their successor $s V \in V_{l+1}$. Although $S_{l}$ is a local minima of $f$ in $G_{l}$, this may not be the case of solution $S_{l-1}$, derived for the upper level $G_{l-1}$, with respect to $G_{l}$. Therefore, a refinement heuristics can be applied to avoid local minima and improve solution quality. Local operations can move the solution towards a lower cost neighboring solution in the search space; for instance, in a community detection problem vertices can be moved between adjacent communities to improve a target quality measure.

\subsection{Related Work}

Early studies of multilevel method were mostly designed to speed up the recursive bisection problem, as its high computational cost prevents wider applicability (BARNARD; SIMON, 1994). One of the first theoretical analysis was presented by Karypis and Kumar (KARYPIS; KUMAR, 1995a), who demonstrated multilevel methods can find high-quality communities in a variety of networks. Later, Karypis and Kumar (KARYPIS; KUMAR, 1998a) introduced the now widely adopted matching algorithms HEM (Heavy Edge Matching), LEM (Light Edge Matching) and MCH (Modified Edge Matching). Other studies relevant for the development and expansion of the multilevel method were conducted by Walshaw and Cross (WALSHAW; CROSS, 1998), who presented a theoretical multilevel formulation of the Kernighan-Lin method for mesh partitioning, and by Korosec et al. (KOROŠEC; ŠILC; ROBIČ, 2003), who introduced a new multilevel colony optimization applicable to several optimization problems. Sciences (WALSHAW, 2004) and Walshaw (WALSHAW, 2008a) provided compelling evidence the multilevel framework is an extremely useful addition to combinatorial optimization toolkits, although it can not be considered a panacea.

Previous work on multilevel method in complex networks can be broadly organized into four categories. A first category encompasses studies that explore features of network domains; e.g., Abou-Rjeili and Karypis (ABOU-RJEILI; KARYPIS, 2006) studied scale-free networks (with power-law degree distribution), Oliveira and Seok (OLIVEIRA; SEOK, 2006) 
designed a multilevel spectral approach that explores features of biological networks (protein complexes) and Valejo et al. (VALEJO et al., 2014a) considered properties of social networks, such as high transitivity and assortativity. A second group comprises contributions focused on scalability, e.g., investigating parallel and distributed paradigms that improve the performance of the coarsening and refinement phases (BARNARD, 1995; KARYPIS; KUMAR, 1996; KARYPIS; KUMAR, 1998c; KARYPIS; KUMAR, 1999b; WALSHAW, 2000; SCHLOEGEL; KARYPIS; KUMAR, 2000; BAÑOS et al., 2004; TRIFUNOVIC; KNOTTENBELT, 2004b; TRIFUNOVIC; KNOTTENBELT, 2004a; ERCIYE; ALP; MARSHALL, 2005; SCHWEITZ; AGRAWAL, 2007; WALSHAW; CROSS, 2007; LASALLE; KARYPIS, 2013; LASALLE; KARYPIS, 2015). A third category of studies is concerned with the optimization of a target objective function, e.g., several contributions focused on improvements in modularity (NEWMAN, 2006; DJIDJEV, 2008; SCHUETZ; CAFLISCH, 2008a; YE; HU; YU, 2008; NOACK; ROTTA, 2009; ROTTA; NOACK, 2011; DJIDJEV; ONUS, 2013; LASALLE; KARYPIS, 2015). Finally, there are significant contributions in applications, including (and not limited to) graph coloring (WALSHAW, 2001b), traveling salesman problem (WALSHAW; EVERETT, 2002), graph drawing (WALSHAW, 2001a), biomedical feature selection (ODUNTAN, 2005), covering design (DAI; LI; TOULOUSE, 2006), DNA sequencing (BLUM; VALLES, 2006), vehicle routing (RODNEY; SOPER; WALSHAW, 2007), semi-supervised learning (ZHANG; WANG, 2010), partitioning or community detection (BICHOT, 2014) and computation of centrality measures (CHERNOSKUTOV; INEICHEN; BEKAS, 2015).

We are not aware of any previous effort concerned with the usage of multilevel methods to solve optimization problems in bipartite networks, despite the relevance of this kind of network to real-world modeling problems. Current multilevel methods cannot be directly applied in bipartite models without adaptations, e.g., they do not consider vertex types, whereas the layers in a bipartite network usually represent distinct types of entities that must be handled independently. For the sake of illustration, suppose a text document collection modeled as a document-word bipartite network. For a start, matching vertices that represent words and vertices that represent documents (i.e., matching of vertices of different layers) would not be meaningful in most application scenarios. Moreover, as the number of words is typically much higher, coarsening the word layer may be sufficient to reduce the asymptotic convergence of a target algorithm.

\subsection{Multilevel method in bipartite networks}

This section introduces a multilevel framework designed to handle bipartite networks. Bearing in mind the previous discussion, we have defined two restrictions that establish the major distinction between current multilevel methods and the one proposed here:

1. Vertices are only allowed to match their set of two-hop neighbors.

2. The matching algorithm must operate on vertices of the same layer. 
Such restrictions support cost-effective implementations of multilevel methods in bipartite networks. From the definitions of bipartite networks and two-hop neighborhoods, the first restriction implies adjacent vertices are not matched. Furthermore, vertices can only match others in their two-hop neighborhood set, rather than any non-adjacent vertex. Considering, for instance, the network in Figure 59, vertex $u_{1}$ can match vertices $u_{2}$ and $u_{3}$, which are non-adjacent and are in $\Gamma_{2}\left(u_{1}\right)$.

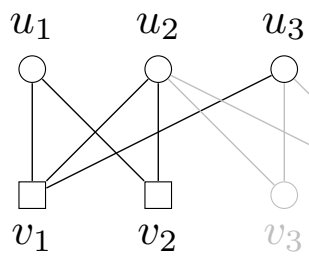

Figure 59 - Example of a bipartite network with $V^{1}=\left\{u_{1}, u_{2}, u_{3}, u_{4}, u_{5}\right\}$ and $V^{2}=\left\{v_{1}, v_{2}, v_{3}, v_{4}\right\}$.

Source: Elaborated by the author.

The first restriction alone does not enforce independent handling of layers, which is guaranteed by the second restriction, which states layers (either one of them or both) will be processed independently in the coarsening phase. Therefore, the coarsening will not match vertices of different kinds and distinct coarsening and refinement algorithms, or distinct parameterizations of the same algorithm may be adopted in processing each layer. Such a restriction also favors the adoption of distributed or parallel processing strategies.

Algorithm 14 summarizes the general multilevel optimization framework for bipartite networks $(\mathrm{MOb})$.

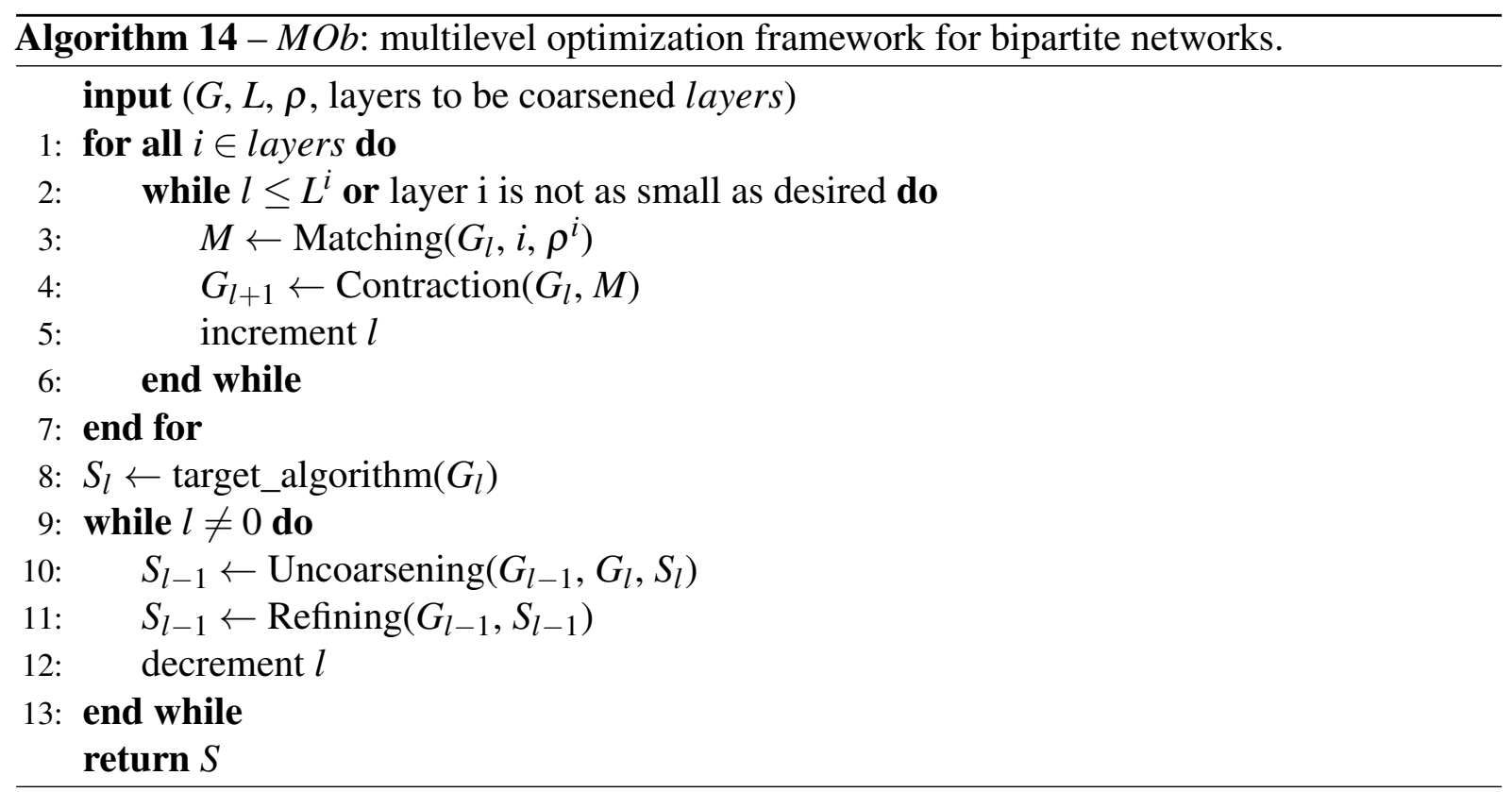

Similarly to the standard multilevel method, it comprises the phases of coarsening (lines 1-4), solution finding (line 8) and uncoarsening (lines 9-11). It takes as inputs the initial bipartite 
network $G=\left(V^{1} \cup V^{2}, E, \sigma, \omega\right)$, and for each layer a maximum number of coarsening levels $L$ and a layer reduction factor $\rho$.

The coarsening is applied to each layer (line 1), level by level until the desired reduction factor is attained, by calls to a matching algorithm (line 3) and a contraction algorithm (line 4). The matching algorithm considers the above restrictions in selecting the vertex pairs to produce the list of independent edges (line 3). The reduction factor $(\rho \in[0,0.5])$ is multiplied by the number of vertices to determine the maximum matching number. If $\rho=0.5$ (the maximum reduction factor), each coarsening iteration will (potentially) reduce the number of vertices by a factor of two, yielding a logarithmic decrease in network size along the process. This is not guaranteed if the network is disconnected or highly sparse, since in this case there may be an insufficient number of edges. The call to the contraction algorithm in the next step (line 4) is responsible for creating the coarsened bipartite network, in which any matched vertex pairs have been merged into super-vertices.

The target algorithm is then executed in the coarsest network $G_{l}$ to obtain a initial solution $S_{l}$ (line 8). Finally, in the subsequent uncoarsening phase this solution is projected back, up to $G_{0}$, through the space of intermediate solutions $S_{l-1}, S_{l-2}, \ldots, S_{1}, S_{0}$ (lines 9-12), possibly refining the solutions at each level (line 11). Although we do not investigate refinement algorithms in this work, the rationale is to apply a local search strategy to improve the current solution, i.e., algorithms should explore small regions of the solution space, in order to reduce impact on performance and scalability. We do introduce novel algorithms for matching, contraction and uncoarsening.

\subsubsection{Matching in bipartite networks}

We propose a straightforward matching algorithm called random greedy matching for bipartite networks $(R G M b)$, described in Algorithm 15, where $S(u, v)$ denotes a similarity function.

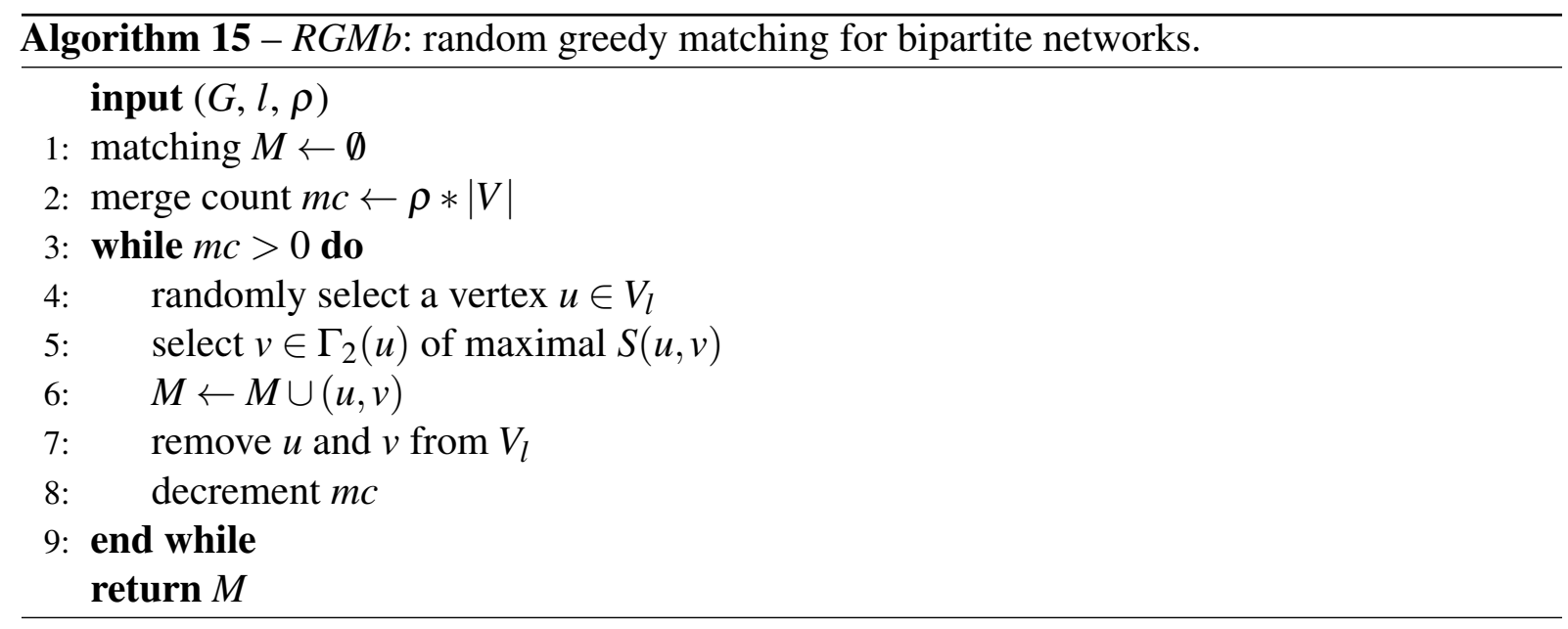

A vertex $u$ is picked randomly (Line 4) at each iteration, and as long as it has not yet been 
matched, one of its unmatched two-hop neighbors $v$ so that $S(u, v)$ is maximal is chosen (Line 5). Vertices $u$ and $v$ are marked as matched and removed from the list of unmatched vertices (Line 7). The process iterates until no more vertices can be eliminated from the list. For unweighted edges, a random neighbor is selected for matching, otherwise, the heaviest adjacent edge is selected.

One may consider different similarity functions, e.g., common neighbors similarity $S_{c n}$ or weighted common neighbors similarity $S_{w c n}$. For illustration, consider a graph $G=$ $\left(V^{1} \cup V^{2}, E\right)$, shown in Figure 60, with $V^{1}=\left\{u_{1}, u_{2}, u_{3}, u_{4}, u_{5}\right\}$ and $V^{2}=\left\{v_{1}, v_{2}, v_{3}, v_{4}, v_{5}\right\}$, $\Gamma_{2}\left(u_{2}\right)=\left\{u_{1}, u_{3}, u_{4}\right\}$ and $u_{1}, u_{2}, u_{3}$ and $u_{4}$ unmatched. Suppose vertex $u_{2}$ has been randomly chosen for matching. Adopting $S_{c n}$ as the similarity function, then $S_{c n}\left(u_{2}, u_{1}\right)=2, S_{c n}\left(u_{2}, u_{3}\right)=3$ and $S_{c n}\left(u_{2}, u_{4}\right)=1$, therefore, pair $\left\{u_{2}, u_{3}\right\}$ would be included in the matching, as illustrated in Figure 60a. Alternatively, for a choice of $S_{w c n}$ as similarity function, $S_{w c n}\left(u_{1}, u_{2}\right)=11$, $S_{w c n}\left(u_{1}, u_{3}\right)=7$ and $S_{w c n}\left(u_{2}, u_{4}\right)=2.5$ and pair $\left\{u_{1}, u_{2}\right\}$ would be included, as illustrated in Figure 60b.

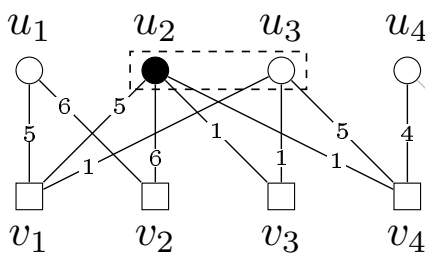

(a)

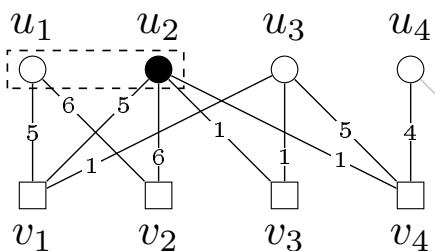

(b)

Figure 60 - Bipartite network, such that $V^{1}=\left\{u_{1}, u_{2}, u_{3}, u_{4}, u_{5}\right\}$ and $V^{2}=\left\{v_{1}, v_{2}, v_{3}, v_{4}\right\}$; (a) pairs $\left\{u_{2}, u_{3}\right\}$ included in the matching; (b) pairs $\left\{u_{1}, u_{2}\right\}$ included in the matching.

Source: Elaborated by the author.

Algorithm $R G M b$ does not ensure an optimal choice over all possible matchings. An alternative strategy would choose from a list of vertex pairs sorted in decreasing order of similarity scores, which may be kept in an appropriate data structure, e.g., a heap or a priority queue. An alternative algorithm that implements this strategy is called greedy sorted matching for bipartite networks (GMb) (Algorithm 16): it selects the best possible match for a vertex from its two-hop neighborhood (line 4).

$G M b$ constructs a priority queue of potential matches ranked by similarity (Lines 3-5) and uses it to retrieve optimal matching choices (Line 9). In case of a tie, it makes a random choice, in order to favor further exploration of the solution space over multiple iterations. If a vertex is selected that has already been matched, it is skipped. GMb, albeit slower than its random search counterpart $R G M b$, is more robust and yields better performance (see Section $5.5)$.

For illustration, consider the graph $G=\left(V^{1} \cup V^{2}, E\right)$ depicted in Figure 61, with $V^{1}=$ $\left\{u_{1}, u_{2}, u_{3}, u_{4}, u_{5}\right\}$ and $V^{2}=\left\{v_{1}, v_{2}, v_{3}, v_{4}, v_{5}\right\}$. Suppose the matching is being performed on layer $V^{1}$. Adopting the $S_{c n}$ similarity, pairs $\left\{u_{2}, u_{3}\right\}$ and $\left\{u_{4}, u_{5}\right\}$ would be included in the matching, as 
illustrated in Figure 61a. Alternatively, for a choice of $S_{w c n}$ similarity, pairs $\left\{u_{1}, u_{2}\right\}$ and $\left\{u_{4}, u_{5}\right\}$ would be included, as shown in Figure 61b.

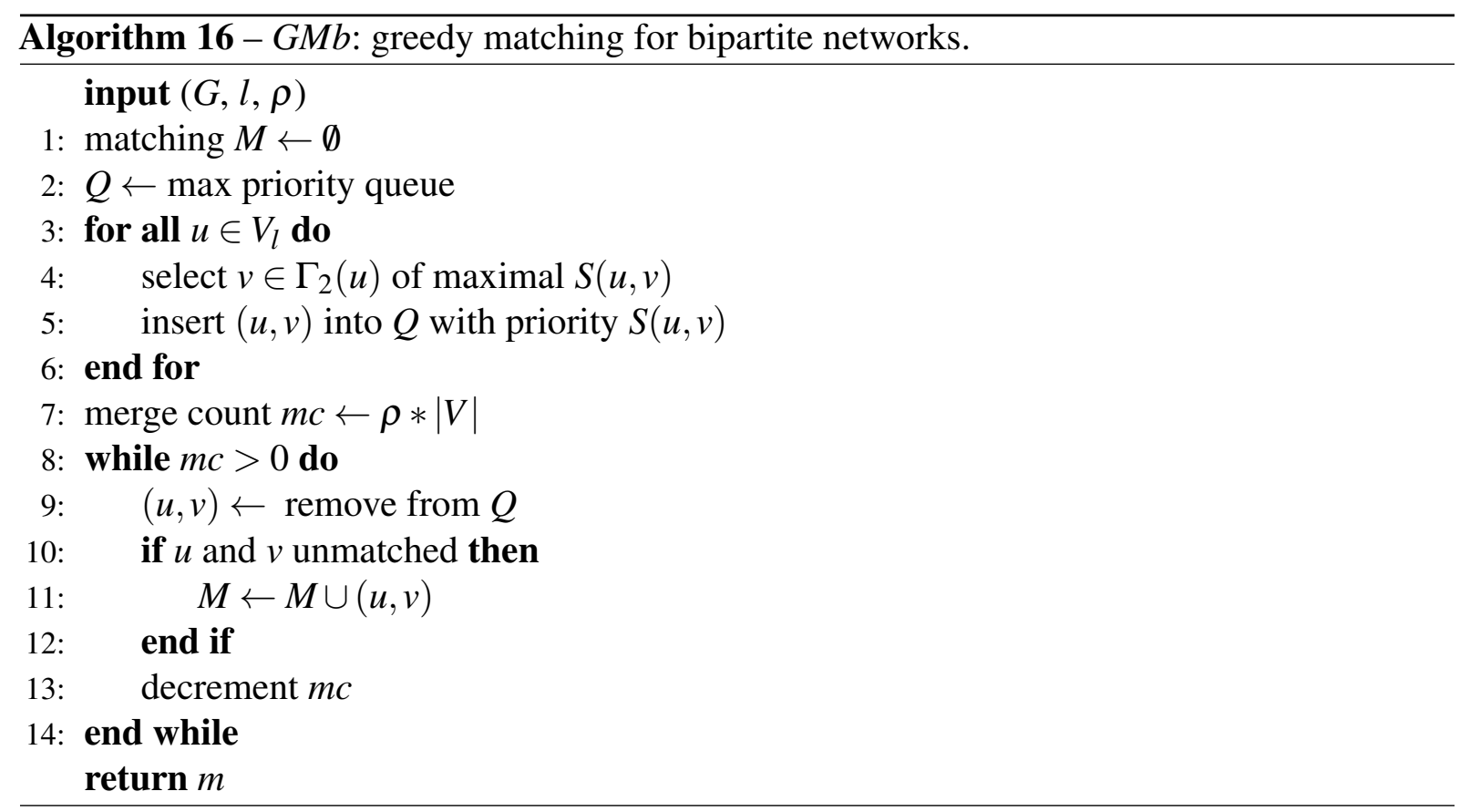

Variations of the general matching algorithm can be obtained depending on the combined choices of matching strategy and similarity function $S(u, v)$. We report four variants, namely $R G M b_{c n}$ and $R G M b_{w c n}$ for a choice of random matching with, respectively, $S_{c n}$ and $S_{w c n}$ similarity; $G M b_{c n}$ and $G M b_{w c n}$ for the equivalent combinations with greedy matching.

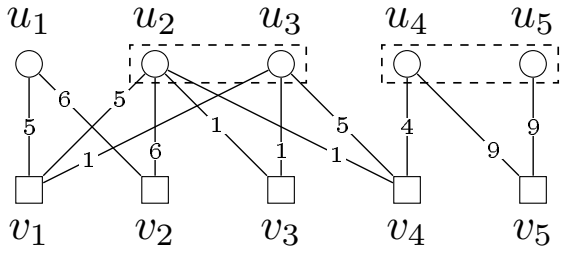

(a)

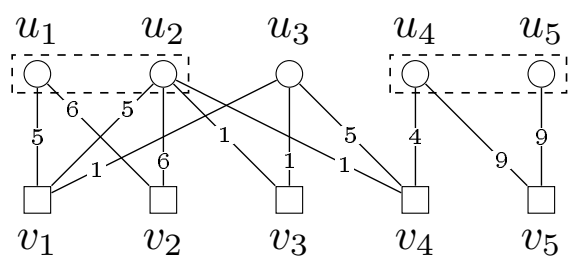

(b)

Figure 61 - Bipartite network, such that $V^{1}=\left\{u_{1}, u_{2}, u_{3}, u_{4}, u_{5}\right\}$ and $V^{2}=\left\{v_{1}, v_{2}, v_{3}, v_{4}\right\}$; (a) pairs $\left\{u_{2}, u_{3}\right\}$ and $\left\{u_{4}, u_{5}\right\}$ included in the matching; (b) pairs $\left\{u_{1}, u_{2}\right\}$ and $\left\{u_{4}, u_{5}\right\}$ included in the matching.

Source: Elaborated by the author.

\subsubsection{Contraction in bipartite networks}

Algorithm 17 ( $\mathrm{Cb}$, contraction of a bipartite network from a matching) describes how to create a coarsened bipartite network from a matching $M$. It takes as input a bipartite network $G_{l}$ and a corresponding matching $M$. First, vertex pairs $\{u, v\} \in M$ are mapped into a successor vector (lines 6-7) and collapsed into a single super-vertex $s V \in G_{l+1}$ (lines 5-10). The successor vector will be accessed in the uncoarsening phase to assign pair $\{u, v\} \in G_{l+1}$ to the same 
subset of its super-vertex $s V$. Any vertices not included in $M$ are inherited by $G_{l+1}$ (line 12 ). After the mapping, adjacent edges in $E_{l}$ are collapsed and added to $E_{l+1}$ (lines 13-20), i.e., each pair $(u, v) \in E_{l}$ holds its successors $(w, z)$ and if edge $(w, z)$ already exists in $E_{l+1}$ its weight is increased by $\omega_{l}(u, v)$ (lines 17-18); otherwise, a new edge $(w, z)$ with weight $\omega_{l}(u, v)$ is inserted into $E_{l+1}$ (lines 19-20).

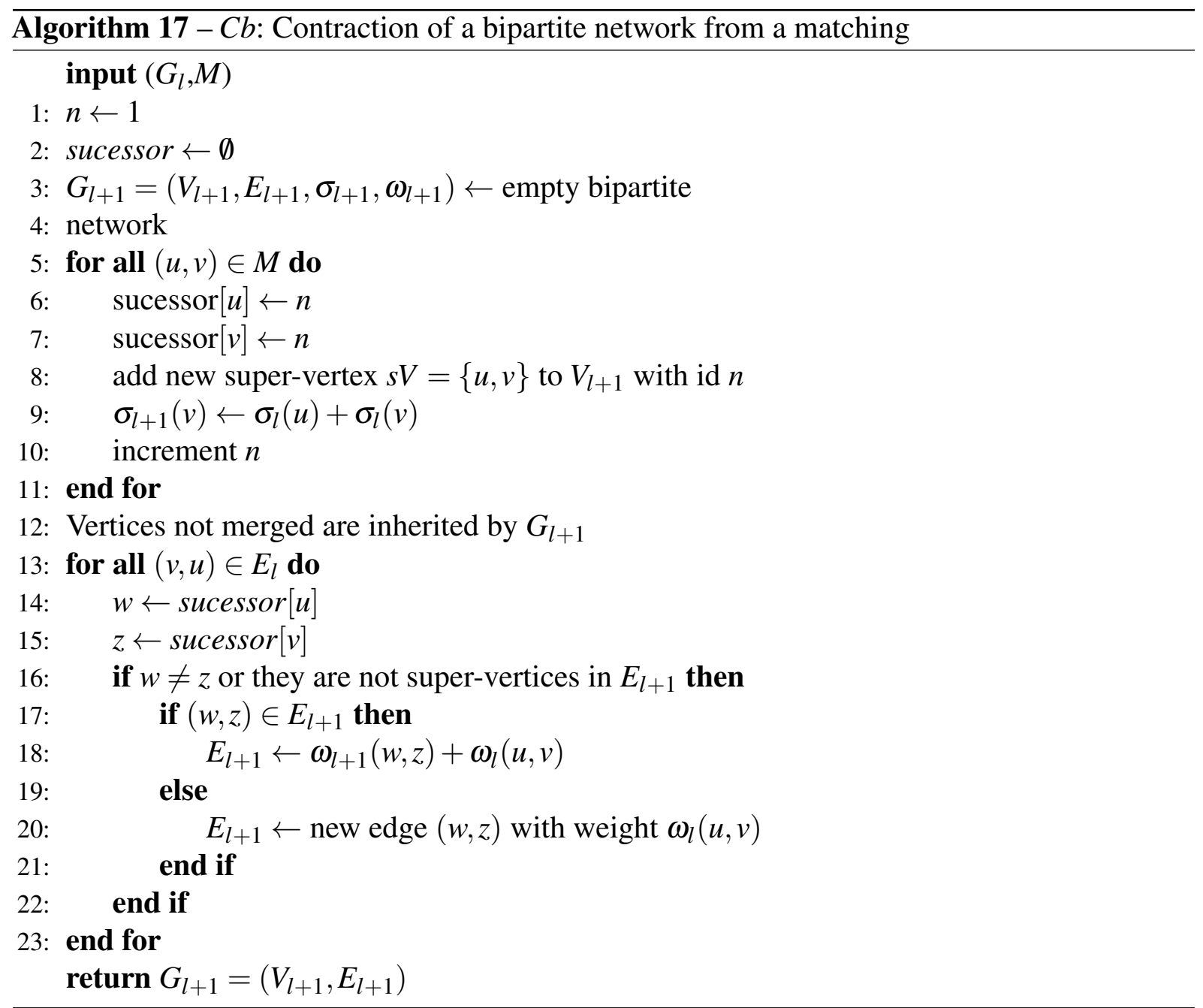

Figure 62 illustrates the contraction process. Considering, for instance, the previous example, in which pairs $\left\{u_{2}, u_{3}\right\}$ and $\left\{u_{4}, u_{5}\right\}$ have been included in the matching, as illustrated in Figure 62a, the resulting coarsened network is depicted in Figure 62b.

The capability of handling layers with distinct parameter settings ( $\rho$ and $L$ ) and coarsening algorithms is a compelling feature of the proposed framework. This is advantageous in many situations, since layers in many real network models are highly unbalanced in size, as in the already mentioned document-word networks. Coarsening just one of the layers is also a useful feature, for example, in dimensionality reduction problems, e.g., to reduce the space of words in document-word networks, as illustrated in Section 5.5.3. 


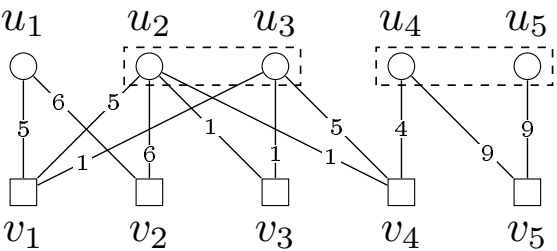

(a)

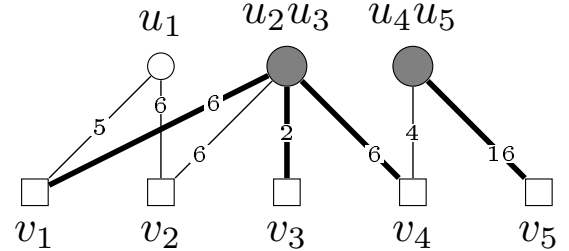

(b)

Figure 62 - Contraction of a layer with the $G M b_{c n}$ matching algorithm: (a) selected matching in original bipartite network; (b) contraction process with newly formed super-vertices and modified edges highlighted.

Source: Elaborated by the author.

\subsubsection{Uncoarsening algorithm}

The solution finding phase executes the target algorithm to obtain a initial solution $S_{L}$. The representation of $S_{L}$ depends on the problem being handled. In a community detection problem, it is described as a partitioning of the vertex set into non-empty partitions $P_{k}$ with $\cup P_{k}=S_{L}, P_{k} \subseteq V_{L}$. Each iteration of the uncoarsening phase projects the current solution, obtained in network $G_{l}$, to network $G_{l-1}$ as described in Algorithm $18 \mathrm{Ub}$ (uncoarsening of bipartite networks). The rationale is, for each vertex $u \in V_{l-1}$ in network $G_{l-1}$ (line 2), to obtain its corresponding successor vertex $w \in V_{l}$ (line 3) and assign vertex $u$ to its partition (lines 4-5). An implementation can keep the successor vector as a global variable, or it may be provided as a network attribute.

As discussed later (Section 5.6), the algorithm can be adapted with little effort to handle other types of problems and representations, e.g., in link prediction, $S$ would be a set of predicted edges $E_{p} \notin E$. In some problems the solution finding and uncoarsening phases are omitted, e.g., in dimension reduction the coarsest bipartite network is itself the final solution (see Section 5.5.3).

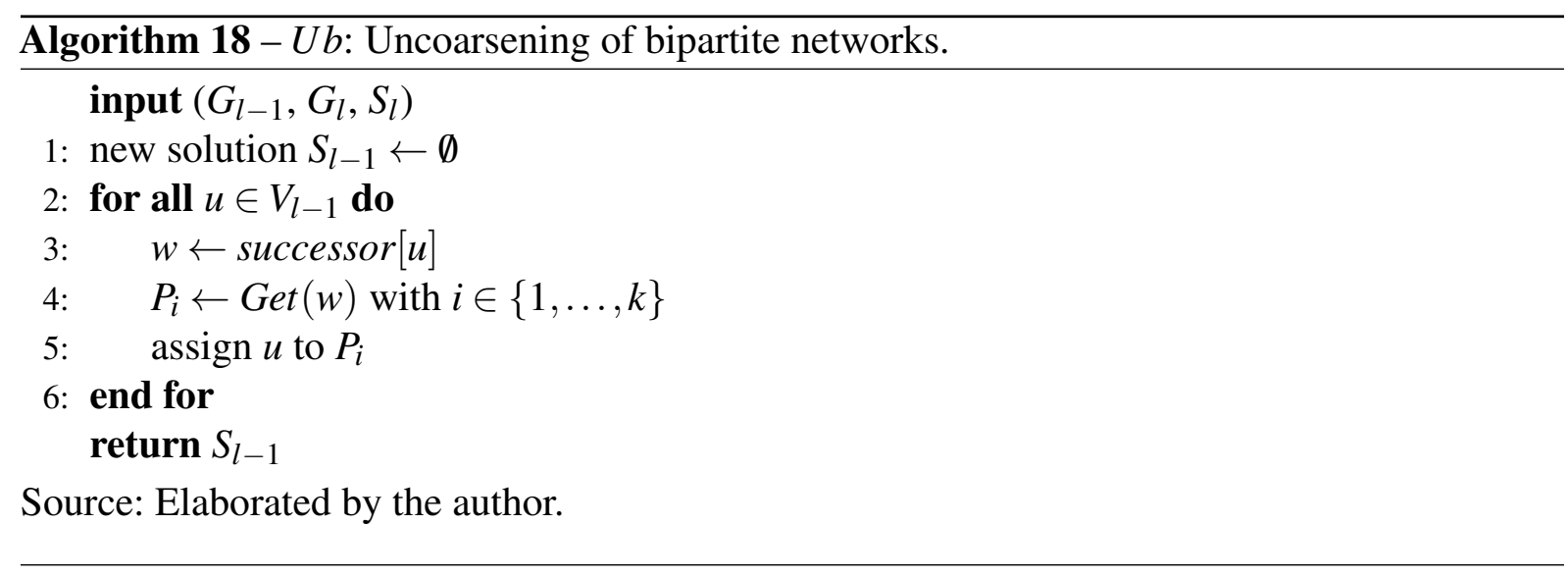




\subsubsection{Computational complexity}

Consider the multilevel method applied to both layers of a network. Computing a matching requires, for each selected vertex $u$, to find its $h$-hop neighborhood $\Gamma_{h}(u)$, which takes $\mathscr{O}(n\langle\kappa\rangle h)$ time, where $\langle\kappa\rangle$ is the average network degree and $h$ specifies the number of distance hops. Most real networks are sparse, i.e., $\langle\kappa\rangle \ll n$ and $m \approx n$. This study only considers two-hop neighborhoods ( $h=2$ ), which leads to notable computational savings, since $\Gamma_{2}$ can be reached in nearly linear time. Appropriate data structures and parallelism can be employed to maximize the efficiency of the matching step (KARPINSKI; RYTTER, 1998; KHAN et al., 2011; KOLLIAS et al., 2014). The subsequent contraction step relies directly on the matching and can be very fast in sparse networks. A coarsened network can be mapped and built in time $\mathscr{O}(|E|)$, since the process is iterated over all edges of its predecessor network.

If the coarsening parameter is set to its maximum value $(\rho=0.5)$, at each iteration the current network size will be reduced at most by a factor of two relative to its predecessor. In this case, given $1<L<n$, the coarsening steps would produce networks of sizes $(n, n / 2, \ldots, n / n)$, yielding a reduction factor $\mathscr{O}\left(\log _{2}(n)\right)$ over the $L$ iterations. For instance, departing from a network with $n=100$ vertices, $\rho=0.5$ and $L=2$, potentially 50 vertex pairs will be merged in the first iteration (from $G_{0}$ to $G_{1}$ ) and 25 in the second (from $G_{1}$ to $G_{2}$ ), a reduction factor of 4. In most real-world problems, such a reduction factor would suffice to produce a manageable model to run the optimization algorithm. As the cost of coarsening decreases drastically at each level, the number of levels $L$ may be neglected in the estimation of the computational cost.

The complexity of the solution finding phase is determined by the target algorithm. If $T_{t g}$ is the cost of the target algorithm on a bipartite network with $n$ vertices, and $T_{s S}$ is the cost to obtain the initial solution, it is not too difficult to show that $T_{s S}=T_{t g} /\left(2^{L}\right)$. Insofar as the hierarchy of approximations produced by coarsening, $L=1$ yields $(n, n / 2), L=2$ yields $(n, n / 2, n / 4), L=3$ yields $(n, n / 2, n / 4, n / 8)$ and so on. Hence, the number of vertices in the coarsest network is approximately $\mathrm{n} /\left(2^{L}\right)$ and therefore $T_{s s}=T_{t g} /\left(2^{L}\right)$. Parameter $L$ can thus be chosen to control the cost deemed acceptable to obtain an initial solution.

As an illustration, consider a network with $n=100$ vertices and two target algorithms, $X$ and $Y$ with $T_{X}=\mathscr{O}(n)$ and $T_{Y}=\mathscr{O}\left(n^{2}\right)$, hence, $T_{X}=\mathscr{O}(100), T_{Y}=\mathscr{O}(10,000)$ and $T_{X}=\sqrt{T_{Y}}$. Consequently, if one wishes to execute algorithm $Y$ on a network with $n=10,000$ with a runtime similar to that of algorithm $X$, the network size should be reduced to $\sqrt{n}=100$, which requires setting $L \approx 7$ and $\rho=0.5$. Of course, the asymptotic complexity of algorithm $Y$ remains quadratic, but the coarsening allows its execution with a runtime equivalent to the linear one.

Analogous to the solution finding phase, the complexity of the uncoarsening phase depends on the target problem (target algorithm), and also on the choice of refinement algorithm, if one is used. In problems such as community detection and classification, it is not necessary to project the solution along the intermediate levels, rather it can be projected directly to the 
original network, since successors have been stored in an array (or other data structure). In these cases, this phase as implemented in Algorithm 3 would have linear cost in the number of vertices, $\mathscr{O}(n)$. As we do not investigate refinement algorithms, an analysis of their asymptotic complexity is suppressed.

\subsection{Experimental Results and Analysis}

We assessed the proposed framework guided by the following research questions:

1. How does coarsening affect a network's topological features at each level?

2. Can the framework improve the efficiency of optimization algorithms without incurring significant losses in the solution quality?

3. How does the framework impact on the scalability of the local search strategy?

4. To which extent can it enable the running of high-cost algorithms on large networks?

5. Is it sufficiently general for handling different combinatorial optimization problems?

We conducted three experimental studies to find answers to the questions. A first study investigated the impact of the coarsening process on the topological properties of a well-known author-paper network. In a second study, the framework was employed in the context of community detection, which is a prototypical application of multilevel methods. Finally, in a third study, it was employed in dimension reduction in a text classification scenario.

The experiments were executed in a Linux machine with 8-core processor with 3.7 GHz CPU and 64 GB main memory. The framework ${ }^{1}$ was implemented in Python with igraph library $^{2}$. We report average values obtained from 30 executions for algorithms that rely on random strategies.

\subsubsection{Analysis of topological properties}

We considered the scientific collaboration network Cond-Mat ${ }^{3}$, which describes coauthorships of preprints posted from 1995 to 1999 in the Condensed Matter section of the arXiv repository, to address the first research question. It has 38,742 vertices (representing authors and papers) and 58,595 edges (co-authorship relations) (additional information can be found elsewhere (NEWMAN, 2001a; NEWMAN, 2001b)). Our interest was to observe how a progressive coarsening affects the intrinsic topological properties of a network.

The Cond-Mat network has been extensively analyzed and is known to have characteristic features regarding degree distribution and clustering coefficients, as depicted in Figure 63.

available from $<$ https://github.com/alanvalejo/mob $>$

available from < http://igraph.org/python/>

available from $<$ https://toreopsahl.com/datasets/\#newman2001> 
According to Figure 63a, the degree distribution follows a power-law relationship characteristic of scale-free networks, with a vast majority of low-degree vertices and a few vertices of very high-degree, i.e., the so-called "hubs".

An inverse relation between vertex clustering coefficients and vertex degrees is also evident in Figure 63b. This is a particular feature of this network: the hubs, i.e., authors with many collaborators, have low-clustered neighborhoods and they tend to interact with collaborators from distinct groups, who are not usually collaborators themselves. In contrast, authors with few collaborators have highly-clustered neighborhoods, i.e., they often interact within smaller and more restrict research groups whose members also collaborate with each other.

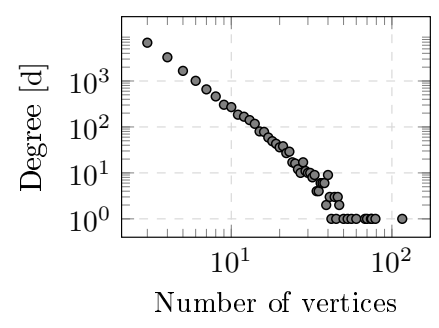

(a)

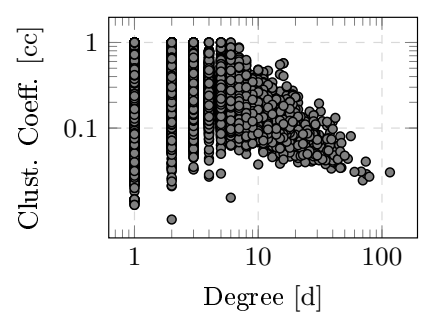

(b)

Figure 63 - Scale-free properties of the Cond-Mat bipartite network. (a): histogram of degree distribution; (b): histogram of two-mode local clustering coefficient.

Source: Elaborated by the author.

We investigated how the scale-free properties of degree distribution and two-mode clustering coefficient are affected as the network is progressively coarsened by matching algorithms $R G M b$ and $G M b$, with input parameters set as $L=10$ and $\rho=0.5$. Figure 64 shows the reduction factor on $|V|=n$ and $|E|=m$ at each level of the coarsening hierarchy, i.e., starting from the original network (level 0) to the coarsest one (level 10). The curves depict the percentages of remaining vertices (left) and edges (right) relative to the initial numbers.

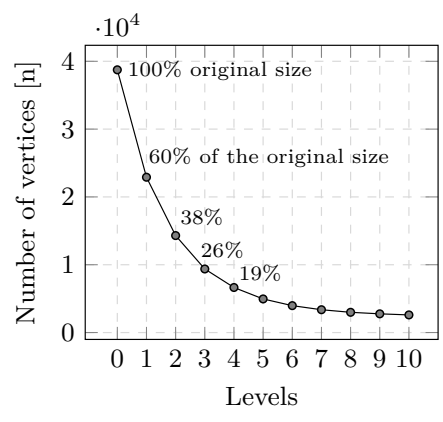

(a)

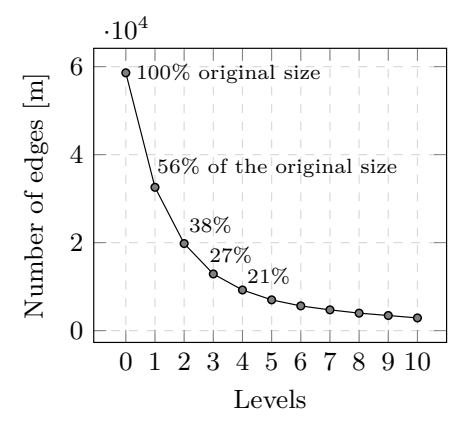

(b)

Figure 64 - Percentages of remaining vertices (left) and edges (right) in the networks along the coarsening hierarchy. 
Figures 65 and 66 show curves for degree distribution and clustering coefficient, respectively, in each coarsened network, from levels 1 to 10 (the top two rows refer to RGMb and the bottom two rows refer to $G M b$ ). Both algorithms yielded very similar distributions of degree and clustering coefficient, which suggests the random exploration of the solution space adopted by $R G M b$ has no strong impact on the topological features of intermediate networks, in comparison with $G M b$.

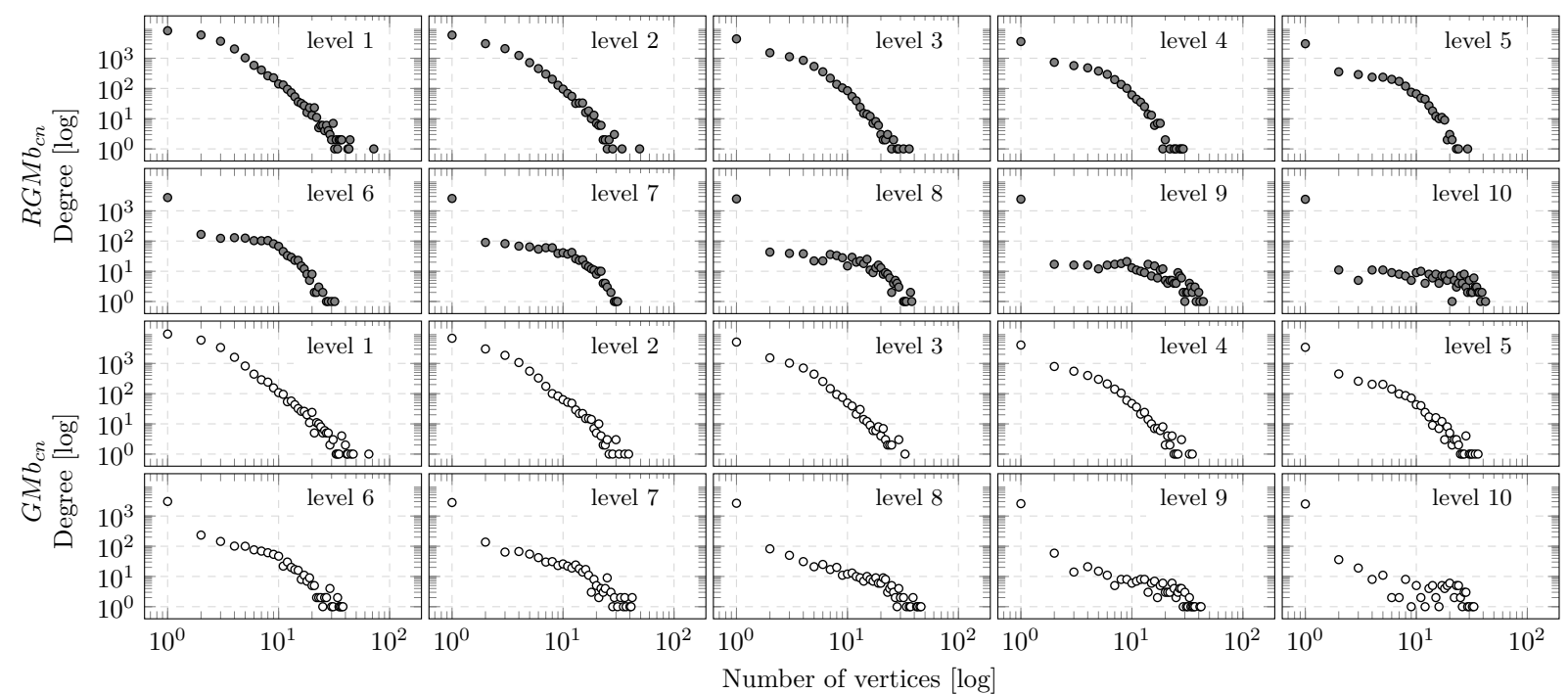

Figure 65 - Degree distribution of the networks at the 10 levels of the coarsening hierarchy $(\rho=0.5)$. Top graphs refer to coarsening with $R G M b$ (random strategy) and bottom graphs refer to coarsening with $G M b$.

Source: Elaborated by the author.

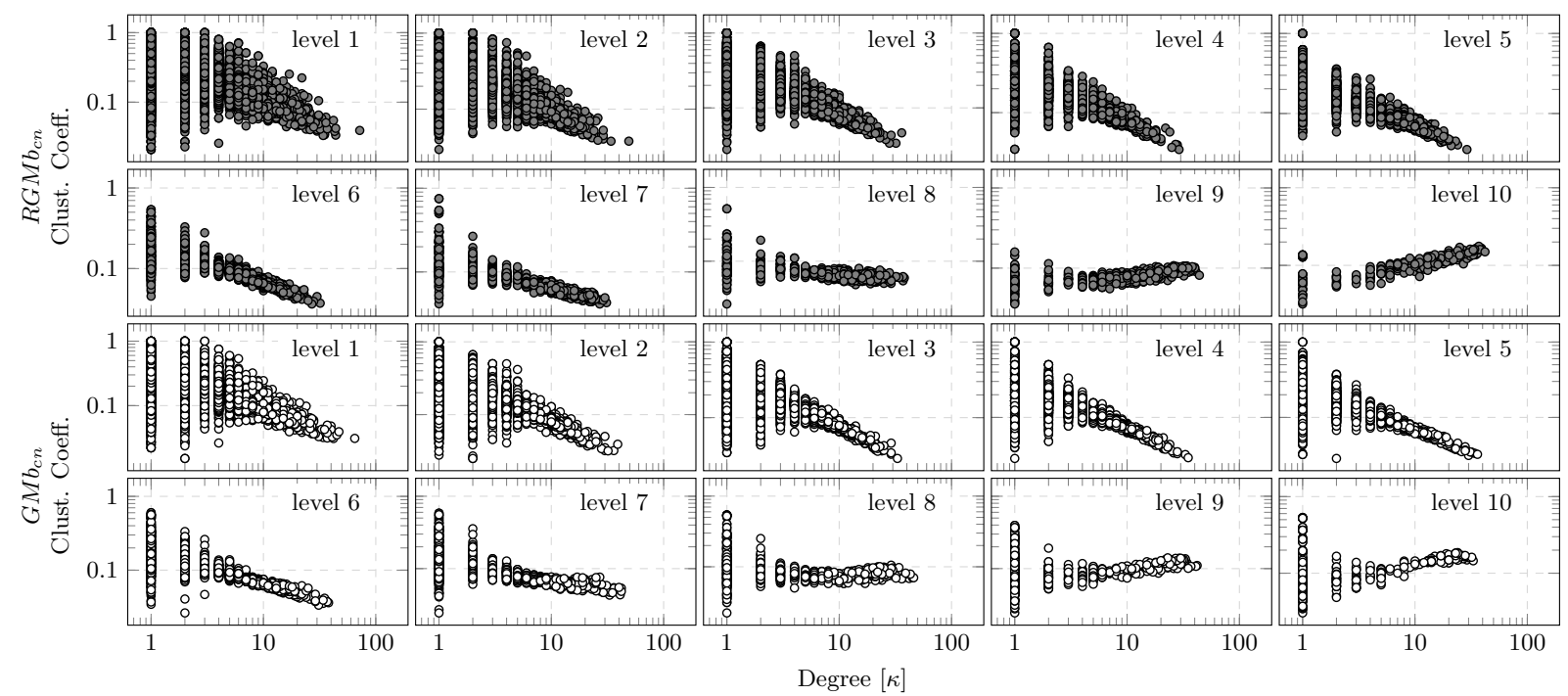

Figure 66 - Two-mode local clustering coefficient of the networks at the 10 levels of the coarsening hierarchy $(\rho=0.5)$. Top graphs refer to coarsening with $R G M b$ (random strategy) and bottom graphs refer to coarsening with $G M b$.

Source: Elaborated by the author. 
The characteristic behavior of degree distribution observed in the original network is reasonably preserved in the coarsened models down to level 3, i.e., coarsened networks at levels 1, 2 and 3 still contain few hubs and many low-degree vertices, a behavior that gradually changes from level 4 onwards.

Particularly from level 8, vertex degrees become more homogeneous, until the original topological features have been completely lost in the final network at level 10. A similar pattern is observed in the clustering coefficient, i.e., again, the original behavior of neighborhoods of hub authors is preserved up to level 3. From level 5 onwards, most vertices converge to lower clustering coefficients and the network's characteristic behavior is completely lost from level 8 .

At the initial coarsening levels (1,2 and 3) only vertices with many two-hop common neighbors can compose a matching. The newly formed super-vertices thus preserve the neighborhood properties of their predecessors, hence, the dominant topological properties of the predecessor network. In other words, it is likely that authors matched at the early coarsening levels indeed have many common collaborators. However, at later levels, authors with few common collaborators may be forced to join, with corrupts the original topological relations in the network. Notice the sizes of networks at levels 9 and 10 were reduced to nearly $10 \%$ of the original network (see Figure 64), which implies the coarsest networks are mostly formed by heavy-weight super-vertices $(\gg \sigma(s V)$ ) sparsely connected. Such super-vertices of low-degree form clustered structures with few triangles.

Results from this analysis indicate a limited reduction of an initial network, up to two or three levels, can preserve its relevant topological features. Furthermore, it is evident that choosing the appropriate coarsening level is critical, and depends on properties of the target application and dataset. Establishing a suitable trade-off between accuracy and runtime may require empirical verification in each case. This inherent limitation can be associated to the well-known overfitting/underfitting problem; however, the coarsening phase in our multilevel process "generalizes" the data instead of an objective function. This may explain why, in some cases, better solutions were obtained on the coarsened networks. In general, extensive coarsening reduces execution times of a target algorithm, but it can lead to excessive generalization of the data with significant degradation of topological features, and possibly algorithm accuracy. In contrast, limited coarsening preserves topological features and accuracy, at the expense of higher execution times.

\subsubsection{Performance in benchmark networks for community detection}

Research efforts on multilevel method have been strongly motivated by community detection (or graph partitioning) problems, which makes this a benchmark problem. Algorithms for community detection in networks split the vertices into disjoint groups (or communities), so as to minimize the number of edges between distinct communities (FORTUNATO, 2010). Barber's modularity optimization (BARBER, 2007) is often employed to identify community 
structures in bipartite networks. Formally, it quantifies the extent of communities formed in both layers relative to a null bipartite network model. Beckett (BECKETT, 2016) introduced the $L P A w b+$ algorithm $^{4}$, which maximizes Barber's modularity through label propagation in weighted bipartite networks and showed it has competitive performance compared with state-ofthe-art methods. However, it is a computationally costly algorithm and becomes unfeasible in large-scale networks.

In order to address the second research question, the $M O b$ framework was tested considering Beckett's algorithm $L P A w b+$ as a target algorithm. Therefore, $M O b$ (Algorithm 14) performs the coarsening, runs $L P A w b+$ to find the community structure in the coarsest network, and projects the solution to obtain the community structure in the original network. Our goal was to investigate whether $M O b$ can yield solutions statistically equivalent in quality to the standard $L P A w b+$, whilst increasing its scalability. Results are compared with those obtained with the standard $L P A w b+$, used as baseline.

We investigated the performance of the four instances of $M O b$, listed in Table 12. They were executed with parameter settings $\rho=0.5$ and $L=[1,2,3]$ in a set of 15 synthetic weighted bipartite networks, identified as R1-R15 (hereafter each $M O b$ instance is referred to by its name).

\begin{tabular}{|c|c|c|c|c|}
\hline \multirow{2}{*}{ Algorithm } & \multicolumn{2}{|c|}{ Coarsening } & \multirow{2}{*}{ Solution finding } & \multirow{2}{*}{ Uncoarsening } \\
\hline & Matching & Contraction & & \\
\hline$M O b-R G M b_{c n}$ & $R G M b$ with $S_{c n}$ & \multirow{4}{*}{$C b$} & \multirow{4}{*}{$L P A w b+$} & \multirow{4}{*}{$U b$} \\
\hline$M O b-R G M b_{w c n}$ & $R G M b$ with $S_{w c n}$ & & & \\
\hline$M O b-G M b_{c n}$ & $G M b$ with $S_{c n}$ & & & \\
\hline$M O b-G M b_{w c n}$ & $G M b$ with $S_{w c n}$ & & & \\
\hline
\end{tabular}

Table $12-M O b$ applied to target algorithm $L P A w b+$ (for the community detection problem): there are two choices of matching algorithm $(R G M b$ and $G M b)$ employed with two choices of similarity function $\left(S_{c n}\right.$ and $\left.S_{w c n}\right)$. All $M O b$ algorithms were executed with $\rho=0.5$ and $L=[1,2,3]$.

Source: Research data.

Synthetic networks were obtained with the community model described in (BECKETT, 2016), which creates unbalanced and randomly positioned community structures. Networks of sizes $n=\left|V^{1}\right|+\left|V^{2}\right|$ were generated within the range $[1,000 ; 15,000]$ at increments of 1,000 and the number of communities was set to $0.01 n$. Edge weights were randomly assigned from a skewed negative binomial distribution and noise was introduced in the connection patterns by reconnecting a percentage of the edges between and within communities.

Performance was measured in terms of accuracy, by means of the NMI (normalized mutual information), which compares the solution found by a selected algorithm with the baseline (LABATUT, 2013), we also measured execution times. Table 13 shows the NMI accuracy values in the 15 networks. The highest values are in bold and values equal to or higher than baseline 
$L P A w b+$ are highlighted with a gray background. The best performances were achieved by $M O b-$ $G M b_{c n}$ with one level of coarsening $(L=1)$ on 11 out of the 15 networks. Baseline $L P A w b+$ yielded the best performance in three networks, whereas $M O b-R G M b_{c n}$ with $L=3$ yielded the worst results.

Limited coarsening levels (mainly $L=1$ ) yielded higher accuracy values, reinforcing that a controlled coarsening can filter the solution space by collapsing promising vertex pairs and removing irrelevant high-cost solutions, while preserving important topological features. In contrast, accuracy deteriorated with more extensive coarsening $(L=3)$, which does not necessarily preserve the original topological properties and is likely to blur the boundaries between adjacent communities. The effect of parameter $L$ depends on network size, i.e.; differences in algorithm accuracy are likely to decrease as the network sizes increase, hinting that higher values of $L$ might be successfully adopted in handling larger networks.

\begin{tabular}{|c|c|c|c|c|c|c|c|c|c|c|c|c|c|c|c|c|}
\hline \multicolumn{2}{|c|}{ Algorithm } & \multicolumn{15}{|c|}{ Dataset } \\
\hline Name & Levels $[L]$ & $\mathrm{R} 1$ & $\mathrm{R} 2$ & R3 & $\mathrm{R} 4$ & R5 & R6 & R7 & R8 & R9 & $\mathrm{R} 10$ & R11 & R12 & R13 & R14 & R15 \\
\hline$L P A w b+$ & 0 & 0.918 & 0.926 & 0.983 & 0.972 & 0.964 & 0.990 & 0.984 & 0.999 & 0.999 & 0.985 & 0.989 & 0.996 & 0.995 & 0.987 & 0.992 \\
\hline$M O b-R G M b_{c n}$ & 1 & 0.984 & 0.985 & 0.983 & 0.988 & 0.990 & 0.991 & 0.992 & 0.991 & 0.991 & 0.992 & 0.992 & 0.992 & 0.991 & 0.991 & 0.992 \\
\hline$M O b-R G M b_{c n}$ & 2 & 0.952 & 0.968 & 0.963 & 0.977 & 0.977 & 0.976 & 0.978 & 0.976 & 0.978 & 0.977 & 0.979 & 0.981 & 0.982 & 0.982 & 0.980 \\
\hline$M O b-R G M b_{c n}$ & 3 & 0.866 & 0.910 & 0.923 & 0.936 & 0.943 & 0.944 & 0.952 & 0.954 & 0.950 & 0.957 & 0.954 & 0.957 & 0.956 & 0.955 & 0.957 \\
\hline$M O b-R G M b_{w c n}$ & 1 & 0.982 & 0.988 & 0.986 & 0.988 & 0.990 & 0.990 & 0.991 & 0.987 & 0.992 & 0.992 & 0.991 & 0.991 & 0.992 & 0.992 & 0.992 \\
\hline$M O b-R G M b_{w c n}$ & 2 & 0.942 & 0.960 & 0.966 & 0.968 & 0.974 & 0.973 & 0.978 & 0.978 & 0.977 & 0.978 & 0.977 & 0.977 & 0.979 & 0.979 & 0.978 \\
\hline$M O b-R G M b_{w c n}$ & 3 & 0.905 & 0.922 & 0.948 & 0.954 & 0.954 & 0.951 & 0.953 & 0.951 & 0.960 & 0.959 & 0.958 & 0.961 & 0.959 & 0.961 & 0.960 \\
\hline$M O b-G M b_{c n}$ & 1 & 0.994 & 0.993 & 0.993 & 0.995 & 0.996 & 0.996 & 0.995 & 0.993 & 0.994 & 0.995 & 0.995 & 0.995 & 0.996 & 0.995 & 0.996 \\
\hline$M O b-G M b_{c n}$ & 2 & 0.981 & 0.986 & 0.982 & 0.983 & 0.988 & 0.987 & 0.988 & 0.987 & 0.988 & 0.988 & 0.988 & 0.988 & 0.988 & 0.990 & 0.990 \\
\hline$M O b-G M b_{c n}$ & 3 & 0.901 & 0.934 & 0.963 & 0.966 & 0.971 & 0.968 & 0.973 & 0.974 & 0.974 & 0.975 & 0.972 & 0.975 & 0.976 & 0.975 & 0.977 \\
\hline$M O b-G M b_{w c n}$ & 1 & 0.990 & 0.989 & 0.992 & 0.992 & 0.995 & 0.993 & 0.992 & 0.992 & 0.993 & 0.994 & 0.995 & 0.995 & 0.995 & 0.994 & 0.994 \\
\hline$M O b-G M b_{w c n}$ & 2 & 0.969 & 0.985 & 0.985 & 0.979 & 0.988 & 0.985 & 0.987 & 0.985 & 0.987 & 0.987 & 0.989 & 0.988 & 0.988 & 0.989 & 0.989 \\
\hline$M O b-G M b_{w c n}$ & 3 & 0.965 & 0.973 & 0.973 & 0.972 & 0.975 & 0.979 & 0.977 & 0.977 & 0.978 & 0.977 & 0.978 & 0.980 & 0.978 & 0.979 & 0.979 \\
\hline
\end{tabular}

Table 13 - NMI accuracy values of all $M O b$ instances and baseline $L P A w b+$ in 15 synthetic networks. The values are average results over 30 executions. The highest accuracy values are in bold and values equal to or higher than the baseline $(L P A w b+)$ are highlighted (gray background).

Source: Research data.

Although $L P A w b+$ achieved the best performance in three networks out of the fifteen, the corresponding accuracy values attained by the $M O b$ instances are very close for these networks. Indeed, in these specific cases accuracy values differ up to 0.006 in R8, up to 0.005 in R9 and up to 0.001 in R12. Interestingly, all $M O b$ instances yielded similar accuracy values and more stable results than the standard $L P A w b+$. The accuracy values obtained with $M O b-G M b_{c n}$ and $L=1$, for instance, are within the range [0.994,0.996], whereas for the standard $L P A w b+$ they are within the range [0.918,0.999], as shown in Figures 67 and 68.

Figure 67 depicts the averages and standard deviations of the accuracy values, whereas Figure 68 shows the dispersion of their distribution and outliers, considering in both cases the alternative settings of parameter $L$. The bar plots in Figure 67a reveal superior performance and 
stability of the four $M O b$ instances when $L=1$, confirmed by their higher average accuracies and narrower standard deviations. When $L=2$, only $M O b-G M b_{c n}$ and $M O b-G M b_{w c n}$ yielded a slightly superior solution in comparison to $L P A w b+$. For $L=3, L P A w b+$ yielded better results than any $M O b$ instance, a consequence of the extensive network reduction.
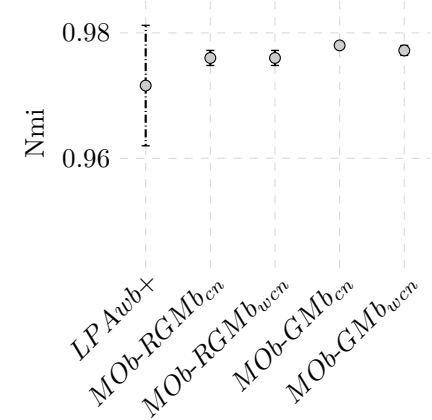

(a) $L=1$
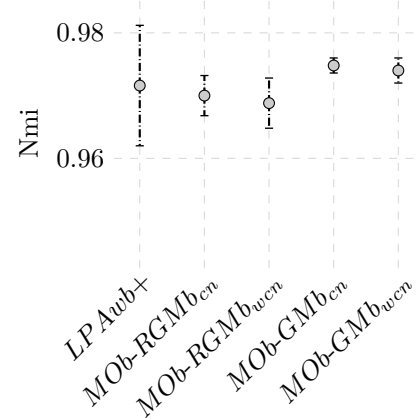

(b) $L=2$

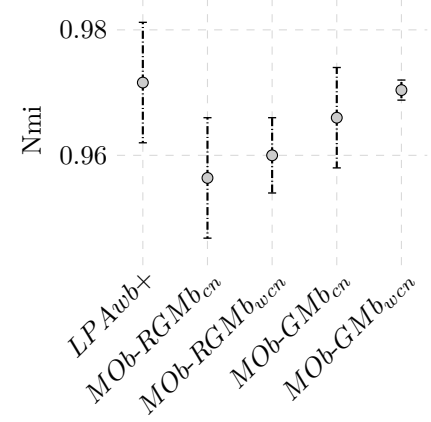

(c) $L=3$

Figure 67 - Averages and standard deviations of the NMI accuracy values obtained with $L P A w b+$ and four $M O b$ instances in three settings of parameter $L$ (number of levels). (a) $L=1$, (67b) $L=2$ and $(67 \mathrm{c}) L=3$.

Source: Elaborated by the author.

The box plots in Figure 68a reveal that for $L=1$ all $M O b$ instances yielded accuracy values within a narrower distribution and higher averages than $L P A w b+$.

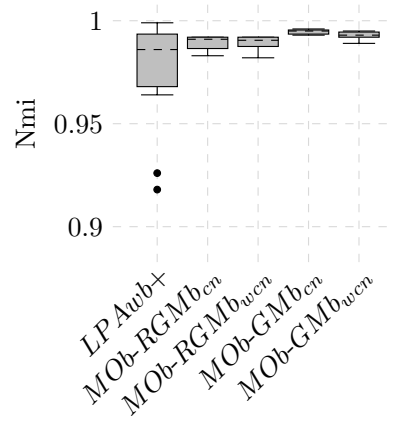

(a) $L=1$

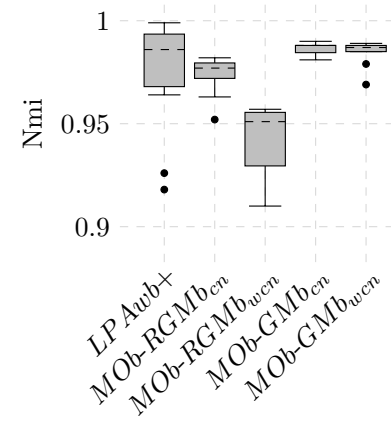

(b) $L=2$

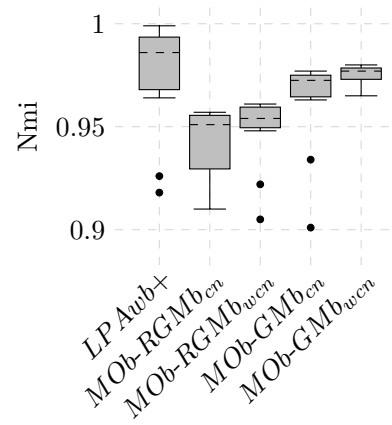

(c) $L=3$

Figure 68 - Shape distribution, variability, and median of the accuracy values yielded by $L P A w b+$ and the four instances of $M O b$ considering three settings of parameter $L$. (a) $L=1$, (67b) $L=2$ and $(67 \mathrm{c}) L=3$.

Source: Elaborated by the author.

We can conclude $M O b$ instances yielded, in general, higher accuracy and improved stability in comparison to $L P A w b+$. In summary, the experimental evidence regarding solution quality (average, standard deviation and dispersion of the accuracy values) suggests the multilevel framework stabilizes and improves the performance of the algorithm.

A Nemenyi post-hoc test (DEMSAR, 2006) was applied to the results in Table 13 to verify statistical differences in the algorithms performances and the results are shown in Figure 
69 for 69 a $L=1,69 \mathrm{~b} L=2$ and $69 \mathrm{c} L=3$. The critical difference (CD) is indicated at the top of each diagram and the algorithms' average ranks are placed on the horizontal axes, with the best ranked algorithms to the left. A black line connects algorithms if no significant difference has been detected among them. According to the the Nemenyi statistics, the critical value for comparing the mean-ranking of two different algorithms at 95 percentile is 1.58 .

Let us consider the outcome of the post-hoc test for $L=1$, i.e., when the number of vertices $n$ is reduced by a factor of two, shown in Figure 69a. $M O b-G M B_{c n}$ was ranked best, followed by $M O b-G M b_{w c n}$ and $M O b-R G M b_{w c n}$ and then $L P A w b+$. Furthermore, $M O b-G M B_{c n}$, $M O b-G M b_{w c n}$ and $M O b-R G M b_{w c n}$ presented statistically significant differences compared with standard $L P A w b+$. Interestingly, for $L=2$ (Figure 69b), $M O b-G M B_{c n}$ and $M O b-G M b_{w c n}$ remain ranked first, however, no statistically significant difference was observed in relation to $L P A w b+$. Finally, for $L=3$ (Figure 69c), $L P A w b+$ was ranked first, with no statistically significant difference observed in relation to $M O b-G M b_{c n}$ or $M O b-G M b_{w c n}$. However, parameter settings $L=3$ and $\rho=0.5$ implied, in this case, in reducing the original size by a factor of $75 \%$, which explains the poor performance of all $M O b$ instances.

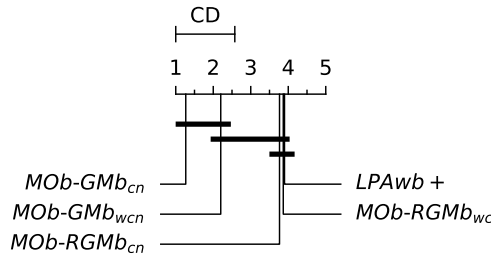

(a) $L=1$

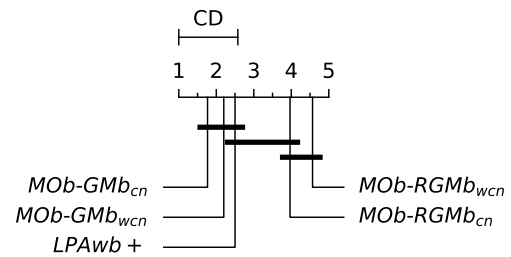

(b) $L=2$

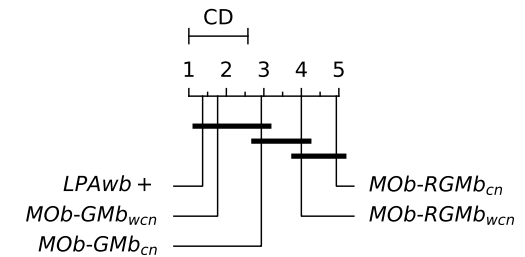

(c) $L=3$

Figure 69 - Nemenyi post-hoc test for $L P A w b+$ and the four $M O b$ instances.

Source: Elaborated by the author.

We also assessed the scalability of the $M O b$ instances to investigate the third and fourth research questions. Their performance was analyzed considering each individual network and the total time spent in the experiments. Table 14 shows the absolute execution times (in seconds) on each network - values refer to the average times from 30 executions of each scenario.

The longest execution time of standard $L P A w b+$ was 302,442 seconds (time to process the largest network) and the shortest was 14 seconds (time to process the smallest one). $M O b$ $G M b_{w c n}$ was the most expensive $M O b$ instance, consuming $(L=1) 25,674$ seconds on the largest network and 3 seconds on the smallest one. Therefore, regarding its maximum and minimum execution times, respectively, $M O b-G M b_{w c n}$ run 11.8 to 4.6 times faster than the standard $L A P w b+$. The maximum and minimum running times of the least expensive $M O b$ instance, $M O b-R G M b_{c n}(L=3)$, were 1,310 seconds and 1 second, respectively. Therefore, $M O b-R G M b_{c n}$ run 230 to 14 times faster than $L A P w b+$.

The analysis in Section 5.5.1 revealed that a network coarsened at level 3 has roughly $25 \%$ of its original size. Let us consider, for example, network R15 $(n=15,000)$ : we know it 
has been reduced to 3,750 vertices at level 3 and algorithm $M O b-R G M b_{c n}$ processed it in 1,310 seconds (see Table 14). This is close to the execution time of standard $L P A w b+$ on network $R 4$ (of size $n=4,000$, similar to the size of R15 coarsened at level 3), i.e., 904 seconds. As $M O b$ $R G M b_{c n}$ executes the coarsening/uncoarsening steps, the actual time spent running $L P A w b+$ to find the solution is roughly similar in both cases, but $M O b-R G M b_{c n}$ is handling a network nearly four times larger.

\begin{tabular}{|c|c|c|c|c|c|c|c|c|c|c|c|c|c|c|c|c|c|}
\hline \multicolumn{2}{|c|}{ Algorithm } & \multicolumn{15}{|c|}{ Dataset } & \multirow{2}{*}{ sum } \\
\hline Name & Levels $[L]$ & R1 & $\mathrm{R} 2$ & R3 & $\mathrm{R} 4$ & R5 & R6 & R7 & R8 & R9 & R10 & R11 & $\mathrm{R} 12$ & R13 & R14 & R15 & \\
\hline$L P A w b+$ & 0 & $\mid 14$ & 96 & 308 & 904 & 2,782 & 2,800 & 7,146 & 15,925 & 39,197 & 56,119 & 66,729 & 75,990 & 97,392 & 224,032 & 302,442 & 891,875 \\
\hline$M O b-R G M b_{c n}$ & 1 & 2 & 10 & 31 & 77 & 160 & 328 & 616 & 1,106 & 1,787 & 4,148 & 5,386 & 8,108 & 10,242 & 15,235 & 23,174 & 70,410 \\
\hline$M O b-R G M b_{c n}$ & 2 & 1 & 3 & 8 & 19 & 42 & 77 & 154 & 267 & 428 & 1,081 & 1,296 & 1,989 & 2,372 & 3,559 & 5,283 & 16,577 \\
\hline$M O b-R G M b_{c n}$ & 3 & 1 & 1 & 4 & 7 & 14 & 25 & 49 & 86 & 142 & 304 & 389 & 586 & 694 & 940 & 1,310 & 4,552 \\
\hline$M O b-R G M b_{w c n}$ & 1 & 3 & 11 & 34 & 79 & 174 & 322 & 643 & 1,424 & 1,715 & 4,192 & 5,335 & 8,582 & 9,355 & 15,121 & 22,535 & 69,525 \\
\hline$M O b-R G M b_{w c n}$ & 2 & 1 & 4 & 9 & 24 & 44 & 101 & 165 & 282 & 460 & 1,049 & 1,399 & 1,911 & 2,245 & 3,281 & 4,866 & 15,840 \\
\hline$M O b-R G M b_{w c n}$ & 3 & 1 & 3 & 6 & 11 & 18 & 33 & 59 & 91 & 139 & 323 & 396 & 522 & 713 & 1,056 & 1,381 & 4,751 \\
\hline$M O b-G M b_{c n}$ & 1 & 2 & 13 & 33 & 78 & 165 & 329 & 623 & 1,111 & 2,680 & 4,145 & 5,561 & 6,705 & 9,091 & 18,136 & 24,732 & 73,402 \\
\hline$M O b-G M b_{c n}$ & 2 & 1 & 5 & 11 & 24 & 46 & 84 & 168 & 298 & 721 & 1,088 & 1,492 & 1,657 & 2,455 & 4,013 & 5,555 & 17,619 \\
\hline$M O b-G M b_{c n}$ & 3 & 1 & 3 & 6 & 13 & 22 & 34 & 62 & 90 & 239 & 332 & 471 & 481 & 672 & 1,180 & 1,596 & 5,204 \\
\hline$M O b-G M b_{w c n}$ & 1 & 3 & 13 & 39 & 83 & 180 & 338 & 688 & 1,135 & 2,864 & 4,392 & 5,819 & 6,317 & 8,932 & 19,016 & 25,674 & 75,493 \\
\hline$M O b-G M b_{w c n}$ & 2 & 3 & 8 & 15 & 32 & 55 & 96 & 187 & 326 & 744 & 1,100 & 1,447 & 1,719 & 2,345 & 4,050 & 4,574 & 16,700 \\
\hline$M O b-G M b_{w c n}$ & 3 & 3 & 7 & 12 & 20 & 31 & 45 & 80 & 117 & 287 & 392 & 469 & 536 & 745 & 1,233 & 1,380 & 5,357 \\
\hline \multicolumn{2}{|l|}{ sum } & 36 & 178 & 514 & 1,371 & 3,733 & 4,613 & 10,639 & 22,257 & 51,403 & 78,664 & 96,189 & 115,104 & 147,253 & 310,853 & 424,502 & $1,267,307$ \\
\hline
\end{tabular}

Table 14 - Absolute runtime (seconds) of $L P A w b+$ and four $M O b$ instances on each network.

Source: Research data.

The total time spent running the experiments was 1,267.307 seconds, or nearly 352 hours. Figure 70 shows the contribution of each algorithm to the total time, considering both absolute values (seconds) 70a and relative values (percentages) $70 \mathrm{~b}$.

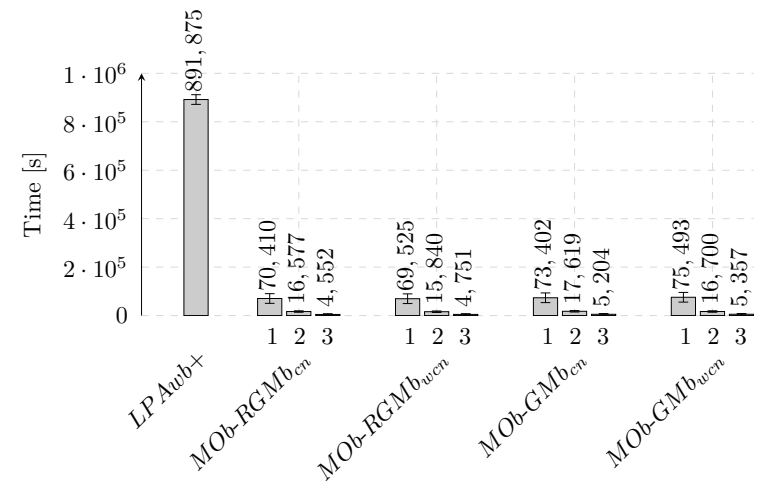

(a) Runtime (seconds)

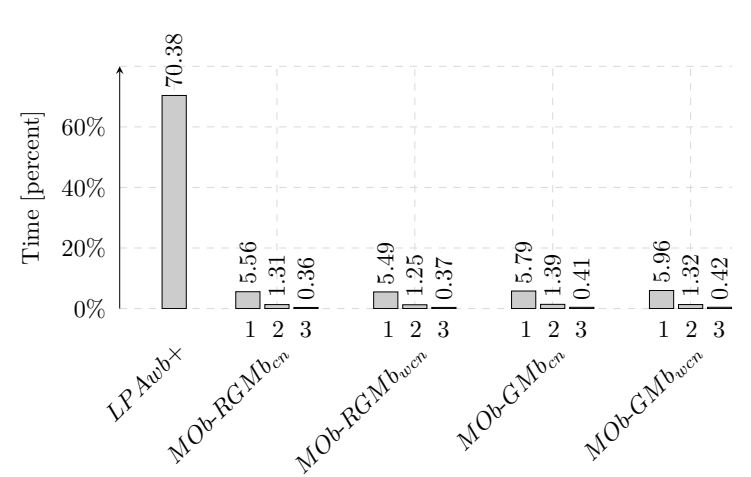

(b) Runtime (percentage)

Figure 70 - Contribution of each algorithm to the total time of the experiments: absolute values (seconds) (a) and percentages (b), 100\% runtime corresponds to 1.127 .300 seconds. Each bar refers to a $M O b$ instance executed with a particular setting of parameter $L=[1,2,3]$, with the specific execution time and standard deviations shown on top in (a) and relative percent times shown on top in (b).

Source: Elaborated by the author. 
In the best case, the $M O b$ instances reduced execution time from 891,875 seconds (nearly 208.8 hours) required by the standard $L P A w b+$ to $4,552.4$ seconds (1.26 hours), which implies $L P A w b+$ was nearly 195 times slower than its $M O b$ instantiations. Executing $L P A w b+$ consumed over $70 \%$ of the time spent in the experiments, whereas roughly $6 \%$ of the time was spent running the $M O b$ instances.

Finally, we assessed the impact of each phase (coarsening, target algorithm on coarsest network and uncoarsening) on the execution time of the multilevel process; we analyzed algorithm behavior separately on each network and then in relation to the total time of the experiments.

The relative contributions of each multilevel phase for each network are shown in Figure 71 (for legibility, we show bars for 12 out of the 15 networks). On the smallest network $(n=1,000)$ the coarsening phase consumed nearly $52 \%$ of the total execution time and the local search step consumed nearly $45 \%$. On the other hand, as the networks increase, the time spent on the coarsening relative to the solution finding gradually decreases. On the larger networks, the coarsening phase consumed roughly $1 \%$ of the total execution time, in contrast to roughly 99\% of the solution finding phase. The time spent on the uncoarsening phase was negligible.

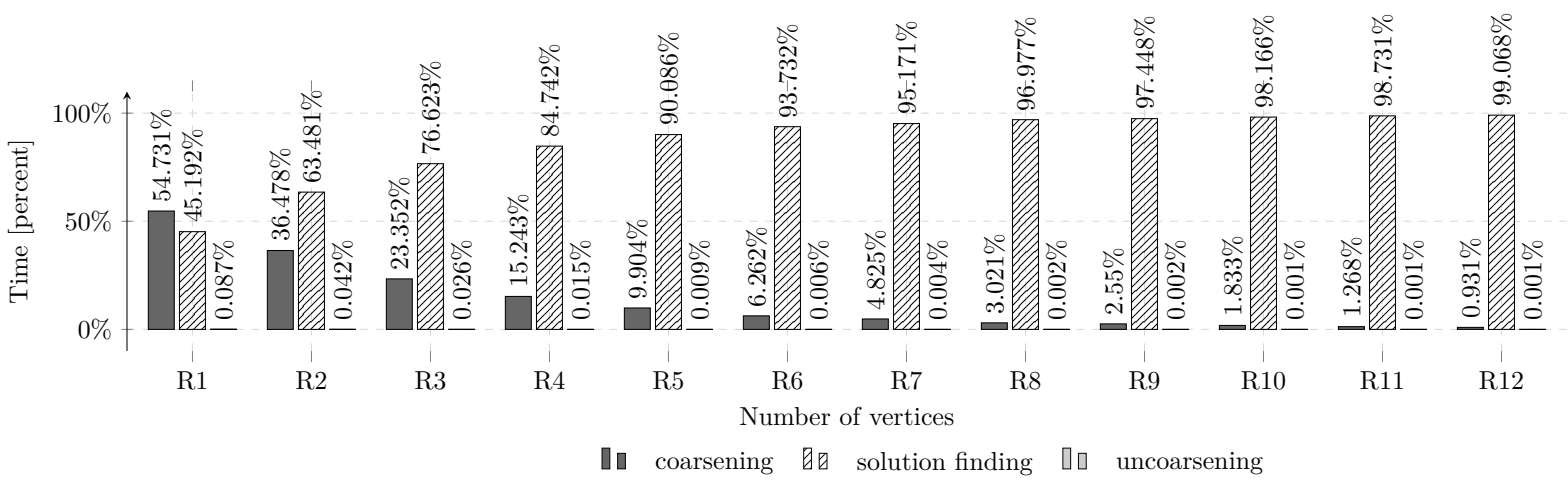

Figure 71 - Relative contribution (percentage) of each multilevel phase to the execution time, on different network sizes $n$ (from 1,000 for R1 to 12,000 for R12).

Source: Elaborated by the author.

Figure 72 shows the relative contribution of each multilevel phase to the total time of the experiments. In general, the coarsening phase consumed less than $1 \%$ of the total execution time, the solution finding phase (executing $L P A w b+$ ) consumed over 99\%, and the time spent in the uncoarsening phase was negligible. These results indicate coarsening and uncoarsening exerted no significant influence on the scalability of the multilevel process and provide empirical evidence the multilevel method is a promising approach to scale network optimization algorithms.

From this empirical investigation we conclude: (i) the proposed $M O b$ approach yielded more accurate and stable results compared to the standard $L P A w b+$; (ii) although solution quality degrades as the network is progressively coarsened, runtime drops drastically at each additional coarsening level; hence, a successful solution requires establishing a suitable trade-off between 
accuracy and execution time.

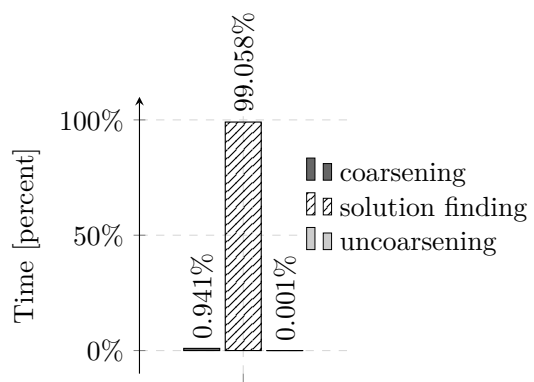

Figure 72 - Average relative contribution (percentage of execution time) of each stage of MOb in all 15 networks.

Source: Elaborated by the author.

\subsubsection{A test case on dimensionality reduction}

We illustrate how $M O b$ framework can be adapted to perform dimensionality reduction in the context of text classification, having the $k$-Nearest Neighbor classifier $(k N N)$ as the target algorithm, in order to exemplify its application in a different kind of optimization problem.

Documents are often represented, in text classification tasks, as multidimensional feature vectors, in which each dimension maps a particular term. As the dimensionality of the representation space has strong impact in classification performance, such tasks are often preceded by a dimension reduction step. Specifically, a $k N N$ classifier that employs a naïve search strategy has time complexity $\mathscr{O}(n d k)$ for a fixed $k$, where $n$ is the cardinality of the training set and $d$ denotes the dimensionality of the document representation.

Alternatively, a document corpus can be represented as a bipartite network $G=\left(\left(V^{1} \cup\right.\right.$ $\left.\left.V^{2}\right), E, \sigma, \omega\right)$, where $V^{1}=\left\{d_{1}, \ldots, d_{r}\right\}$ is the set of documents and $V^{2}=\left\{t_{1}, \ldots, t_{s}\right\}$ is the set of terms. An edge $(u, v)$ exists if term $t_{u}$ occurs in document $d_{v}$, and the term's frequency determines the corresponding edge weight $w(u, v)$. Such a network is represented as a bi-adjacency matrix $A_{r \times s}$, where $r=\left|V^{1}\right|, s=\left|V^{2}\right|$ and $A_{u, v}=w(u, v)$ if $(u, v) \in E$. Dimensionality reduction is aimed at obtaining a lower dimensional matrix $A_{r^{\prime} \times s^{\prime}}^{\prime}$, with $r^{\prime}=r$ and $s^{\prime} \gg s$.

The proposed solution is described in Algorithm 19, multilevel dimensionality reduction (Mdr), which only requires a coarsening phase (lines 1-3). It takes as inputs the initial bipartite network $G$, the term layer $t_{l}$ to be coarsened, the desired maximum number of levels $L$ and reduction factor $\rho$, and returns the bi-adjacency matrix of the coarsest network (line 5).

We adopted $R G M b_{c n}$ as the contraction algorithm in the $M d r$ implementation, and used $M d r$ in combination with a $k N N$ classifier, assuming $k=3$ and the Euclidean distance as similarity measure, hereafter referred to as $M d r-k N N$. The results obtained considering a reduction factor $\rho=0.5$ and $L$ varying in the range $[1,2,3,4,5,6,7,8,9,10]$ were compared to an equivalent $k N N$ setting that employed PCA (Principal Components Analysis) (JOLLIFFE, 
2002) for dimensionality reduction $(P C A-k N N)$. The complexity of $P C A$ is $\mathscr{O}\left(d^{2} n+d^{3}\right)$, where $n$ denotes the number of samples and $d$ the data dimensionality.

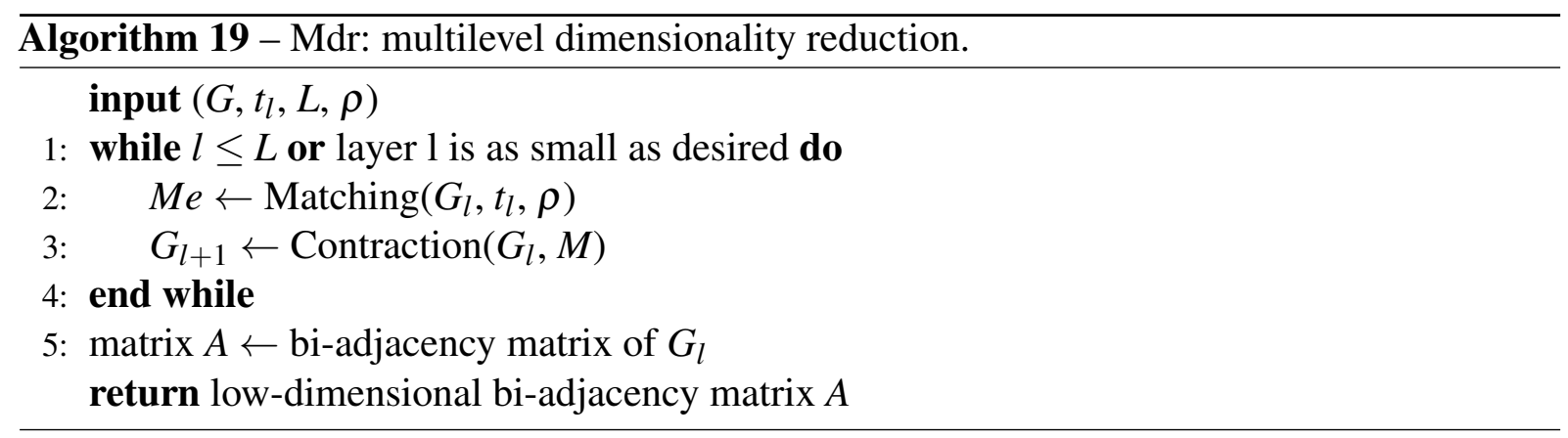

Implementations $M d r-k N N$ and $P C A-k N N$ were used to classify thirteen real documentterm networks available from the literature, described in Table 15, considering a cross-validation with permutation testing and ten-fold cross-validation to estimate classification error. The training set was randomly split into ten equal-sized subsets, so the classification model was trained in nine subsets and tested on the remaining one.

\begin{tabular}{|l|c|c|c|c|c|c|c|c|c|c|c|c|c|}
\cline { 2 - 12 } \multicolumn{1}{c|}{} & cstr & oh0 & oh5 & 0h10 & syskillwebert & opinosis & classic4 & hitech & industry & irish & la1s & enron & acm \\
\hline Documents & 299 & 1,003 & 918 & 1,050 & 334 & 6,457 & 7,095 & 2,301 & 8,817 & 1,660 & 3,204 & 13,199 & 3,493 \\
\hline Terms & 1,726 & 3,183 & 3,013 & 3,239 & 4,340 & 2,693 & 7,749 & 10,000 & 10,000 & 8,659 & 10,000 & 10,000 & 10,000 \\
\hline Domain & Scientific & Medical & Medical & Medical & Web pages & Sentiment & Scientific & News & Web page & Sentiment & News & E-mail & Scientific \\
\hline
\end{tabular}

Table 15 - Properties of text collections considered.

Source: Research data.

Figure 73 shows the performance of the $M d r-k N N$ classifier as a function of the number of levels in the coarsening hierarchy, where $L=0$ refers to classification with no dimensionality reduction. Figures 73(a), (b) and (c) show accuracy, dimensionality of the representation (number of terms) and execution times, respectively.

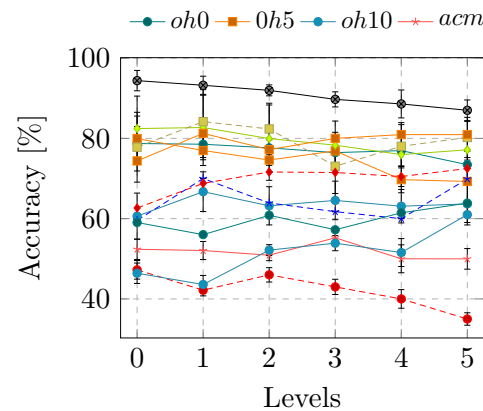

(a)

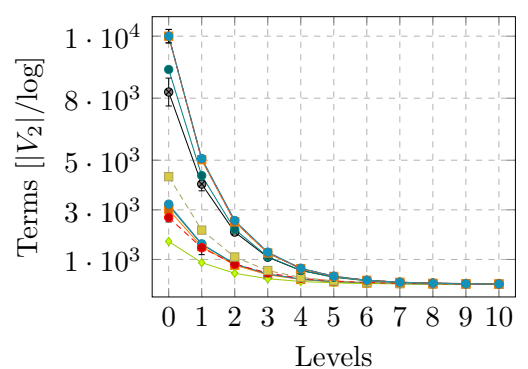

(b)

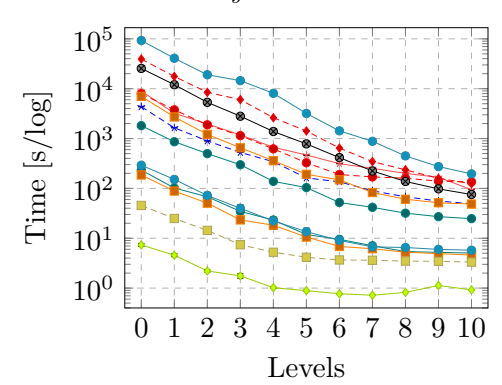

(c)

Figure 73 - Performance (accuracy values) of the $M d r-k N N$ classifier. $L=0$ corresponds to classification with no dimension reduction applied.

Source: Elaborated by the author.

A moderate decrease in accuracy values is observed up to level $L=5$. In general, accuracy is stable at the early coarsening levels, which suggests the first coarsening iterations have limited 
impact in solution quality, as it is more likely that highly correlated terms are being matched. Figure 73(a) shows accuracies up to level 5. Moreover, since from level 5 onwards the coarsening yields no significant reduction in the number of terms it may be interrupted at this point. Also, a sharp reduction in execution times is observed only up to this level. We remind the analysis presented in Section 5.5.1, which showed a network coarsened at level 5 has roughly $15 \%$ its original size.

We compared the classification accuracies of $k N N$ without dimension reduction and $M d r$ $k N N$ (with coarsening at $L=[1,3,5]$ ) and those of $P C A-k N N$ (with equivalent dimensionality reduction). The results are shown in Table 16. The highest accuracy values are shown in bold and values equivalent or superior to baseline $k N N$ are shown with a gray background. $M d r-k N N$ achieved the best performance in five out of the thirteen networks; $P C A-k N N$ performed best in seven networks and standard $k N N$ performed best in only one of them.

\begin{tabular}{|l|c|c|c|c|c|c|c|}
\hline \multirow{2}{*}{ Dataset } & \multirow{2}{*}{$\mathrm{kNN}$} & \multicolumn{2}{|c|}{$L=1$} & \multicolumn{2}{c|}{$L=3$} & \multicolumn{2}{c|}{$L=5$} \\
\cline { 3 - 8 } & & $M d r+k N N$ & $P C A-k N N$ & $M d r-k N N$ & $P C A-k N N$ & $M d r-k N N$ & $P C A-k N N$ \\
\hline \hline cstr & $82.44 \pm 8.09$ & $82.71 \pm 8.04$ & $75.86 \pm 7.15$ & $80.10 \pm 6.10$ & $79.31 \pm 2.02$ & $78.30 \pm 3.20$ & $\mathbf{8 6 . 2 1} \pm 7.10$ \\
\hline oh0 & $78.72 \pm 6.84$ & $81.15 \pm 3.31$ & $\mathbf{8 5 . 0 0} \pm 3.98$ & $78.95 \pm 2.02$ & $76.00 \pm 4.31$ & $75.90 \pm 3.32$ & $76.00 \pm 6.20$ \\
\hline oh5 & $80.27 \pm 5.09$ & $78.83 \pm 3.73$ & $76.92 \pm 4.31$ & $76.88 \pm 4.17$ & $\mathbf{8 1 . 3 2} \pm 3.11$ & $69.27 \pm 3.89$ & $74.36 \pm 5.35$ \\
\hline oh10 & $60.62 \pm 5.75$ & $66.92 \pm 4.95$ & $\mathbf{7 5 . 2 4} \pm 4.21$ & $64.54 \pm 5.04$ & $72.38 \pm 3.29$ & $64.38 \pm 2.16$ & $73.33 \pm 4.23$ \\
\hline opinosis & $47.25 \pm 2.32$ & $42.18 \pm 1.42$ & $43.57 \pm 1.20$ & $45.90 \pm 4.92$ & $\mathbf{5 5 . 1 9} \pm 8.15$ & $35.98 \pm 3.06$ & $49.15 \pm 5.78$ \\
\hline syskillwebert & $77.80 \pm 8.70$ & $\mathbf{8 7 . 9 1} \pm 6.57$ & $87.88 \pm 5.14$ & $75.91 \pm 3.65$ & $75.06 \pm 6.45$ & $80.23 \pm 2.12$ & $78.00 \pm 5.65$ \\
\hline acm & $52.37 \pm 2.54$ & $52.96 \pm 2.26$ & $54.44 \pm 2.62$ & $\mathbf{5 5 . 2 3} \pm 3.15$ & $53.00 \pm 2.98$ & $49.98 \pm 1.87$ & $49.10 \pm 4.87$ \\
\hline hitech & $59.57 \pm 3.55$ & $\mathbf{7 0 . 0 0} \pm 2.59$ & $64.35 \pm 3.24$ & $61.74 \pm 2.34$ & $66.10 \pm 3.10$ & $69.90 \pm 2.55$ & $66.90 \pm 3.50$ \\
\hline industry & $62.66 \pm 5.60$ & $71.62 \pm 3.05$ & $66.86 \pm 1.98$ & $71.50 \pm 6.12$ & $\mathbf{7 8 . 3 0} \pm 4.12$ & $72.89 \pm 3.34$ & $75.20 \pm 4.32$ \\
\hline irish & $59.04 \pm 4.28$ & $63.86 \pm 2.34$ & $57.83 \pm 4.32$ & $58.23 \pm 3.87$ & $56.90 \pm 4.07$ & $\mathbf{6 3 . 9 1} \pm 5.12$ & $63.10 \pm 5.98$ \\
\hline la1s & $74.38 \pm 6.74$ & $81.25 \pm 4.10$ & $\mathbf{8 3 . 7 5} \pm 5.20$ & $80.00 \pm 4.12$ & $82.12 \pm 2.23$ & $80.94 \pm 4.80$ & $82.30 \pm 4.76$ \\
\hline classic4 & $\mathbf{9 4 . 3 9} \pm 6.04$ & $93.82 \pm 2.55$ & $93.51 \pm 3.15$ & $89.72 \pm 4.01$ & $75.87 \pm 2.68$ & $87.00 \pm 3.12$ & $91.90 \pm 3.28$ \\
\hline enron & $46.40 \pm 5.18$ & $52.16 \pm 5.09$ & $47.76 \pm 2.98$ & $53.89 \pm 2.90$ & $55.00 \pm 3.77$ & $\mathbf{6 1 . 0 0} \pm 2.14$ & $60.10 \pm 6.12$ \\
\hline
\end{tabular}

Table 16 - Accuracy values obtained with standard $k N N, M d r-k N N$ and $P C A-k N N$. The highest accuracy values are shown in bold and values equivalent or superior to the baseline $k N N$ are highlighted in gray.

Source: Research data.

A Nemenyi post-hoc test was applied to the results in Table 16 in order to detect statistical differences among the algorithms. Demsar post-hoc test requires each algorithm and dataset to be independent, therefore, we perform the dimensionality reduction at each level separately. The results are shown in Figure 74 for $74 \mathrm{a} L=1,74 \mathrm{~b} L=2$ and $74 \mathrm{c} L=3$.

According to Nemenyi statistics, in all diagrams, the critical value for comparing the mean-ranking of two different algorithms at 95 percentile is 0.92 . No significant difference was observed between the algorithms, therefore, they are connected by a bold line in each diagram. Albeit differences are not significant, Figure 74a shows $M d r-k N N$ was ranked best for $L=1$, whereas for $L=2$ and $L=3 P C A-k N N$ was ranked best (Figures $74 \mathrm{~b}$ and $74 \mathrm{c}$ ). Therefore, $M d r-k N N$ proved competitive in terms of accuracy, in comparison to $P C A-k N N$ and baseline 
$k N N$.

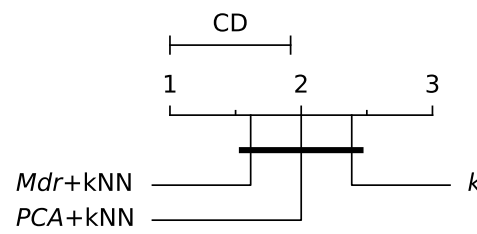

(a) $L=1$

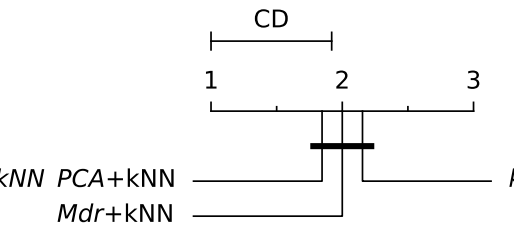

(b) $L=3$

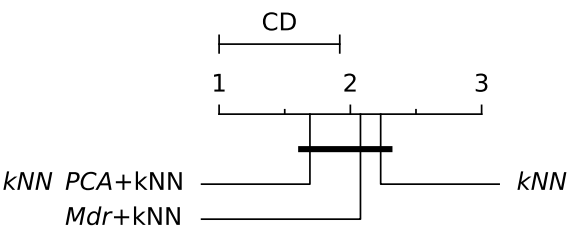

(c) $L=5$

Figure 74 - Nemenyi post-hoc test for $k N N$ and its variant with $M d r$ and $P C A$ for dimensionality reduction in three settings of parameter $L$. (a) $L=1$, (b) $L=3$ and (c) $L=5$.

Source: Elaborated by the author.

This case study has been presented as a preliminary investigation on the feasibility of extending the proposed multilevel framework to other combinatorial problems beyond community detection. The $M d r$ algorithm deserves further consideration and could incorporate additional capabilities, e.g., it would be convenient to reduce the feature space to a target dimensionality, rather than by a given reduction factor. Furthermore, the coarsening algorithm could take into account intrinsic characteristics of specific kinds of document-term networks by means of customized matching algorithms. Moreover, $M d r$ can be employed in connection with other classification algorithms.

\subsection{The application of $M O b$ to other combinatorial opti- mization problems}

It is relatively straightforward to apply $M O b$ to several combinatorial optimization problems beyond community detection. For instance, in Section 5.5.3 we illustrated its application to handle a dimensionality reduction problem over a bipartite network in which the two vertex layers represent objects and features, respectively. In this context, only the feature layer was coarsened and the reduced feature space is given directly by the adjacency matrix of the coarsest network. Likewise, it can be easily instantiated to handle overlapping or fuzzy community detection or classification problems.

In overlapping community detection, for each decomposed $s V \in V_{l+1}$, its original vertices $\{u, v\} \in V_{l}$ are assigned to the same set of communities as the corresponding super-vertex. If the coarsened network has a fuzzy structure, the strength of a vertex' pertinence to a community will be equal to that of its super-vertex. Similarly, in a classification problem original vertices $\{u, v\} \in V_{i}$ should be assigned to the same class of their super-vertex. Therefore, instantiating the framework to handle either problem would require just minor modifications in the projection algorithm (Algorithm 18).

$\mathrm{MOb}$ might also be useful to support interactive visualization of large-scale bipartite 
networks, by means of navigation over a hierarchy of coarsened networks, which would demand a data structure to keep these intermediate networks.

Instantiation to other scenarios is not necessarily as straightforward, and may require further modifications in the proposed algorithms. For example, in the edge clustering problem (also called link communities) the contraction algorithm used in coarsening phase would require adjustments. Whereas in community detection a vertex inherits the same group assignment of its super-vertex, here each edge must inherit the connections from its super-edge. Therefore, an edge $e \in E_{l+1}$ incident to $s_{V} \in V_{l+1}$ must refer to the edges incident to vertices $\{u, v\} \in V_{l}$. Algorithm 17 does not implement this function; however, it could be done by keeping an additional data structure similar to the successor vector.

Another possible application is in link prediction or recommendation problems, albeit this poses a more complex scenario for generalization. Link prediction methods rely on similarity between vertices, since similar vertices are likely to share common links. However, such information is not explicitly given for the super-vertices in a coarsened bipartite network. Nonetheless, the framework could be employed to reduce the number of required operations, i.e. super-vertices might be created grouping vertices with shared $h$-hop neighbors, thus filtering the search space. Link prediction could be performed in the uncoarsening phase through the decomposed super-vertices, and the solution finding and uncoarsening phases would be executed simultaneously.

As a final consideration, the matching algorithm used for coarsening should be carefully designed to incorporate the specific characteristics of each problem and context.

\subsection{Conclusion and further research}

Inspired by the potential of general-purpose multilevel methods to scale optimization algorithms we have introduced algorithms of a novel multilevel optimization framework for bipartite networks $(\mathrm{MOb})$, and illustrated its application on two combinatorial optimization problems. Our framework accounts for the specificities of bipartite networks and provides a powerful tool for handling a variety of problems.

We investigated three empirical scenarios to illustrate strengths and limitations of the proposed $M O b$ framework. A first study has shown that a controlled coarsening preserves relevant topological features of a network. A second study described an application in community detection, showing that $M O b$ combined with a proper local search strategy can drastically improve speedup of a classic community detection algorithm while preserving solution quality. Finally, in a third study we considered text classification to illustrate how the general framework can be instantiated to handle different combinatorial optimization problems.

Our results provide compelling evidence that $M O b$ offers a competitive approach to scale 
existing methods while preserving solution quality, and reinforce its usefulness for handling combinatorial optimization problems in large bipartite networks. Furthermore, the framework is flexible and can be adjusted to incorporate alternative and novel coarsening methods targeted at specific applications. We also discuss some general guidelines for future applications of combinatorial problems, such as link prediction, edge clustering, and interactive graph visualization.

Identifying the level of coarsening that will yield a suitable trade-off between accuracy and execution times is a critical issue in applying the proposed multilevel method. Currently, this is done by means of empirical investigation in each application problem and dataset, but it certainly deserves further investigation.

We also plan as future work to extend the framework to handle problems defined in heterogeneous networks, where edges connect vertices of multiple types. It would be applicable, e.g., to document-word networks indicating associations of the type document-word, wordword, and document-document; or networks describing relations between words, documents and authors. We are also interested in investigating distributed or parallel paradigms, as well as in application of $M O b$ to supervised and unsupervised classification tasks. An implementation of the general framework is currently available and can be downloaded from <https://github.com/ alanvalejo/mob $>$.

\section{Appendix}

\section{A Supplemental material}

The appendix contains supplemental empirical analysis. $O P M$ (one-mode projectionbased), described in Section 4 and in Valejo et al. (2017), Valejo et al. (2018b), was empirically compared to the results obtained by $M O b$ (based directly on the bipartite structure). Similar to the methodology conducted with $M O b$ (described in Section 5.5.2), $O P M$ was tested considering Beckett's algorithm $L P A w b+$ as a target algorithm. Therefore, OPM (Algorithm 14) performs the coarsening, runs $L P A w b+$ to find the community structure in the coarsest network, and, finally, projects the solution to obtain the community structure in the original network. OPM was executed with parameter settings $\rho=0.5$, using HEM, GHEM and RM matching algorithms and $L=[1,2,3]$ in the set of 15 synthetic weighted bipartite networks, identified as R1-R15, used as benchmark data in the Section 5.5.2. Table 13 shows the NMI accuracy values in 15 networks. The highest values are in bold. The best performances were achieved by $M O b-G M b_{c n}$ with one level of coarsening $(L=1)$ in all networks.

A Nemenyi post-hoc test (DEMSAR, 2006) was applied to the results in Table 17 to verify statistical significance of the observed differences in algorithm performances. Results are shown in Figure 75, in which (a) and (b) depict, respectively, the ranks for $L=1$ and $L=3$. The critical difference (CD) is indicated at the top of each diagram and algorithms' average ranks are 
placed in the horizontal axes, with the best ranked placed left. A black line connects algorithms if there is no significant performance difference. According to the Nemenyi statistic, the critical value for comparing the mean-ranking of two different algorithms at 95 percentile is 2.33 for (a) and (b). In both cases $G M b_{c n}$ and $G M b_{w c n}$ was ranked best, followed by $O P M_{g h e m}$ and $O P M_{h e m}$. $O P M_{r m}$ obtained the worst rank.

\begin{tabular}{|c|c|c|c|c|c|c|c|c|c|c|c|c|c|c|c|c|}
\hline \multicolumn{2}{|c|}{ Algorithm } & \multicolumn{15}{|c|}{ Dataset } \\
\hline Name & Levels $[L]$ & $\mathrm{R} 1$ & $\mathrm{R} 2$ & $\mathrm{R} 3$ & $\mathrm{R} 4$ & R5 & R6 & R7 & $\mathrm{R} 8$ & R9 & R10 & R11 & R12 & R13 & R14 & R15 \\
\hline$M O b-R G M b_{c n}$ & 1 & 0.984 & 0.985 & 0.983 & 0.988 & 0.990 & 0.991 & 0.992 & 0.991 & 0.991 & 0.992 & 0.992 & 0.992 & 0.991 & 0.991 & 0.992 \\
\hline$M O b-R G M b_{c n}$ & 2 & 0.952 & 0.968 & 0.963 & 0.977 & 0.977 & 0.976 & 0.978 & 0.976 & 0.978 & 0.977 & 0.979 & 0.981 & 0.982 & 0.982 & 0.980 \\
\hline$M O b-R G M b_{c n}$ & 3 & 0.866 & 0.910 & 0.923 & 0.936 & 0.943 & 0.944 & 0.952 & 0.954 & 0.950 & 0.957 & 0.954 & 0.957 & 0.956 & 0.955 & 0.957 \\
\hline$M O b-R G M b_{w c n}$ & 1 & 0.982 & 988 & 0.986 & 0.988 & 0.990 & 0.990 & 0.991 & 987 & 0.992 & 0.992 & 0.991 & 0.991 & 0.992 & 0.992 & 0.992 \\
\hline$M O b-R G M b_{w c n}$ & 2 & 0.942 & 0.960 & 0.966 & 0.968 & 0.974 & 0.973 & 0.978 & 0.978 & 0.977 & 0.978 & 0.977 & 0.977 & 0.979 & 0.979 & 0.978 \\
\hline$M O b-R G M b_{w c n}$ & 3 & 0.905 & 0.922 & 0.948 & 0.954 & 0.954 & 0.951 & 0.953 & 0.951 & 0.960 & 0.959 & 0.958 & 0.961 & 0.959 & 0.961 & 0.960 \\
\hline$M O b-G M b_{c n}$ & 1 & 0.994 & 0.993 & 0.993 & 0.995 & 0.996 & 0.996 & 0.995 & 0.993 & 0.994 & 0.995 & 0.995 & 0.995 & 0.996 & 0.995 & 0.996 \\
\hline$M O b-G M b_{c n}$ & 2 & 0.981 & 0.986 & 0.982 & 0.983 & 0.988 & 0.987 & 0.988 & 0.987 & 0.988 & 0.988 & 0.988 & 0.988 & 0.988 & 0.990 & 0.990 \\
\hline$M O b-G M b_{c n}$ & 3 & 0.901 & 0.934 & 0.963 & 0.966 & 0.971 & 0.968 & 0.973 & 0.974 & 0.974 & 0.975 & 0.972 & 0.975 & 0.976 & 0.975 & 0.977 \\
\hline$M O b-G M b_{w c n}$ & 1 & 0.990 & .989 & 0.992 & 0.992 & 0.995 & 0.993 & 0.992 & 0.992 & 0.993 & 0.994 & 0.995 & 0.995 & 0.995 & 0.994 & 0.994 \\
\hline$M O b-G M b_{w c n}$ & 2 & 0.969 & 0.985 & 0.985 & 0.979 & 0.988 & 0.985 & 0.987 & 0.985 & 0.987 & 0.987 & 0.989 & 0.988 & 0.988 & 0.989 & 0.989 \\
\hline$M O b-G M b_{w c n}$ & 3 & 0.965 & 0.973 & 0.973 & 0.972 & 0.975 & 0.979 & 0.977 & 0.977 & 0.978 & 0.977 & 0.978 & 0.980 & 0.978 & 0.979 & 0.979 \\
\hline OPM ghem & 1 & 0.992 & 0.982 & 0.985 & 0.990 & 0.991 & 0.993 & 0.990 & 0.992 & 0.991 & 0.995 & 0.991 & 0.991 & 0.994 & 0.990 & 0.995 \\
\hline$O P M_{\text {ghem }}$ & 2 & 0.981 & 0.987 & 0.980 & 0.986 & 0.991 & 0.989 & 0.988 & 0.991 & 0.988 & 0.989 & 0.991 & 0.990 & 0.991 & 0.989 & 0.993 \\
\hline OPM ghem & 3 & 0.875 & 0.952 & 0.960 & 0.964 & 0.963 & 0.971 & 0.972 & 0.975 & 0.973 & 0.975 & 0.973 & 0.974 & 0.976 & 0.976 & 0.976 \\
\hline$O P M_{\text {hem }}$ & 1 & 0.991 & 0.987 & 0.985 & 0.984 & 0.991 & 0.990 & 0.985 & 0.992 & 0.991 & 0.995 & 0.990 & 0.991 & 0.992 & 0.991 & 0.993 \\
\hline$O P M_{h e m}$ & 2 & 0.973 & 0.985 & 0.981 & 0.982 & 0.989 & 0.987 & 0.989 & 0.989 & 0.988 & 0.989 & 0.989 & 0.988 & 0.989 & 0.990 & 0.991 \\
\hline$O P M_{\text {hem }}$ & 3 & 0.873 & 0.952 & 0.960 & 0.966 & 0.963 & 0.971 & 0.972 & 0.975 & 0.972 & 0.975 & 0.973 & 0.974 & 0.975 & 0.976 & 0.976 \\
\hline$\overline{O P M_{r m}}$ & 1 & 0.312 & 0.358 & 0.409 & 0.412 & 0.407 & 0.414 & 0.442 & 0.462 & 0.448 & 0.462 & 0.468 & 0.483 & 0.483 & 0.502 & 0.498 \\
\hline$O P M_{r m}$ & 2 & 0.146 & 0.169 & 0.147 & 0.135 & 0.158 & 0.171 & 0.157 & 0.162 & 0.150 & 0.150 & 0.150 & 0.161 & 0.148 & 0.152 & 0.161 \\
\hline$O P M_{r m}$ & 3 & 0.100 & 0.119 & 0.119 & 0.098 & 0.105 & 0.105 & 0.090 & 0.079 & 0.082 & 0.069 & 0.078 & 0.099 & 0.084 & 0.082 & 0.072 \\
\hline
\end{tabular}

Table 17 - NMI accuracy values of the $M O b$ and $O P M$ instantiations in 15 synthetic networks (averages over 30 executions for $O P M_{h e m}$ and $O P M_{r m}, M O b-R G M b_{c n}$ and $M O b-R G M b_{w c n}$, in contrast, $O P M_{g h e m}, M O b-G M b_{c n}$ and $M O b-G M b_{w c n}$ are deterministic). We highlight the highest accuracy values, shown in bold.

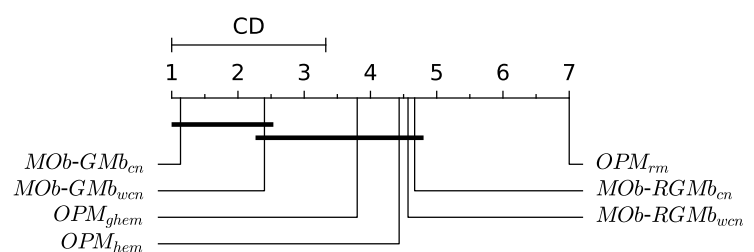

(a) $L=1$

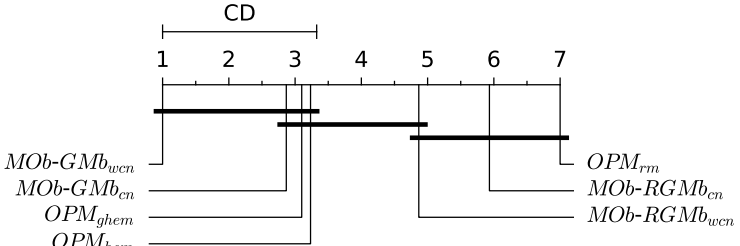

(b) $L=3$

Figure 75 - Nemenyi post-hoc test for $M O b$ and $O P M$ versions of the $L P A w b+:$ (a) $L=1$ and (b) $L=3$. Source: Elaborated by the author.

The results indicate the direct use of the bipartite structure performs better than the use 
of a one-mode projection-based strategy. Therefore, although $O P M$ reach good performance, it should be used with care, since some information can be lost in the one-mode projection process. For instance, in an author-paper network, many papers are written by a single author, and this information is lost in the one-mode projection since only collaborations are taken into account. 
CHAPTER

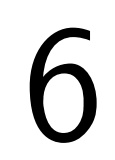

(1)

\section{A COARSENING METHOD FOR LABELED AND UNLABELLED BIPARTITE NETWORK VIA WEIGHT-CONSTRAINED LABEL PROPAGATION}

A multilevel method is a scalable strategy to solve optimization problems in large bipartite networks which operates in three stages: Initially, the input network is iteratively coarsened into a hierarchy of gradually smaller networks. Coarsening implies in collapsing vertices into so-called super-vertices, which typically inherit properties of their originating vertices. The target algorithm is then executed in the coarsest network to obtain an initial solution, which will be successively projected back over the inverse sequence of coarsened networks up to the initial network. Despite its potential, the strategy faces theoretical and practical limitations. Coarsening is usually attained with pairwise matching of vertices following a user-defined criterion, an approach that renders the network reduction process extremely slow, and possibly yields degraded solutions due to propagation of poor matches. Additionally, the super-vertices do not necessarily preserve relevant properties, and proper parameterization of coarsening algorithms is difficult. We address these issues with a near-linear complexity coarsening strategy based on weight-constrained label propagation. Our strategy collapses groups of vertices, rather than pairs, yielding faster and more extensive network reduction. Moreover, users can specify the desired size of the coarsest network and control super-vertex weights. The applicability of our proposal is illustrated in multiple scenarios, namely: multilevel implementation of an existing high-cost community detection algorithm; as a direct algorithm for community detection; finally, network visualization, in connection with force-directed graph drawing algorithms. Results provide empirical evidence on the potential of the proposed coarsening strategy to foster novel applications of the multilevel method in bipartite networks. 


\subsection{Introduction}

Bipartite, or two-layer, networks comprise a particular category of networks in which the set of vertices is split into two disjoint sub-sets (the layers) and edges can connect only vertices of different layers. This type of network often results from modeling pairwise relationships between two categories of entities, e.g. documents and terms (FALEIROS; ROSSI; LOPES, 2017), patient and gene expression (or clinical variables) (HWANG et al., 2008), individuals and songs (or books, or films) (GRUJIĆ, 2008), scientific papers and their authors (NEWMAN, 2001a; NEWMAN, 2001b). In the last years, there has been a growing scientific interest in bipartite networks given their occurrence in many data analytics problems, such as community detection, text classification, and dimensionality reduction (VALEJO et al., 2018a; ROSSI et al., 2014). Their potential emerges from the ability to explicitly show conceptual links between different types of entities and their derived latent features, which supports a new perspective in modeling complex systems. Solving these and other problems requires finding a minimum (or maximum) cost in a solution space wherein the number of possible states is finite and usually exponential. Indeed, many theoretical problems defined in bipartite networks have been proven to be $\mathscr{N} \mathscr{P}$-complete or $\mathscr{N} \mathscr{P}$-hard (ASRATIAN; DENLEY; HäGGKVIST, 1998).

Recent contributions investigated the multilevel method as a scalable strategy to solve partitioning and other hard problems in bipartite networks (VALEJO et al., 2017; VALEJO et al., 2018a; VALEJO et al., 2018b). Problem-solving with a multilevel strategy comprises three phases. In an initial phase the input network is iteratively coarsened into a hierarchy of gradually smaller networks. The size of the hierarchy and graph are controlled by the number of levels and reduction factor, respectively. This phase, called coarsening, implies in collapsing pairs of vertices and its incident edges into so-called super-vertices and super-edges, which typically inherit properties of their originating vertices and edges. In a second phase, the problem is solved executing the target algorithm in the coarsest network. Finally, in a third phase, the initial solution is successively projected back over the inverse sequence of coarsened networks, until obtaining a solution relative to the original network. The three phases and the algorithms used therein are discussed in detail in Section 6.2.1.

Despite its potential, the multilevel method faces theoretical and practical limitations. One such limitation is that network reduction is often attained by collapsing vertex pairs according to a defined criterion. This pairwise collapsing may degrade the process due to poor choices at early stages being propagated into further coarsening levels. Besides, pairwise collapsing implies in the network being reduced by up to half its original size on a single iteration, thus reducing very large networks can be extremely slow. As an additional limitation, creating super-vertices balanced or size-constrained relative to user-defined properties is unfeasible with existing coarsening algorithms. Finally, most algorithms require as input parameters a number of coarsening levels and a network reduction factor, which may be difficult to set a priori. It would be simpler to inform the number of vertices expected in the coarsest network. These limitations are discussed 
in detail in Section 6.2.2.

In this work we address these issues in order to enhance the applicability of the multilevel method and foster the development of novel multilevel solutions for problems defined in bipartite networks. This paper brings a two-fold contribution, from both a theoretical and an applied perspectives:

1. We introduce a near linear coarsening strategy based on weight-constrained label propagation. Compared with existing solutions its major distinguishing features are: (i) it allows collapsing groups of vertices, rather than vertex pairs; (ii) it yields higher network reduction (on the order of 60\%-90\%) at each coarsening level; and (iii) it allows specifying the target reduction either by informing the number of coarsening levels and corresponding reduction factors or, alternatively, by informing the desired number of vertices in the coarsest network.

2. We illustrate multiple applications of the proposed coarseing strategy. We used it in a multilevel implementation of the high-cost bipartite Stochastic Block Model (biSBM) (LARREMORE; CLAUSET; JACOBS, 2014) community detection algorithm. Results from an empirical investigation conducted on a representative set of synthetic and real-world networks show a considerable speed up with no significant loss in solution quality, as compared to established coarsening algorithms. We also show the strategy is easily adaptable for community detection in bipartite networks, yielding partitions that are statistically equivalent, regarding accuracy, to those obtained with well-known algorithms. Finally, we discuss the potential applicability of the coarsening strategy for network visualization, illustrating its usage in connection with network drawing algorithms to obtain informative layouts of large bipartite networks.

The remainder of the paper is organized as follows: in Section 6.2 we introduce the basic concepts and notation and provide an overview of the multilevel approach applied in bipartite networks, with a critical discussion of current limitations. In Section 6.3 we introduce the coarsening strategy and its implementation. Empirical results are reported in Section 6.5, covering a benchmark analysis of our proposal; an empirical study of its application in community detection, and an investigation of its potential to support visualization of large networks. Finally, in Section 6.6 we summarize our findings and discuss future work.

\subsection{Background}

A network $G=(V, E, \sigma, \omega)$ is bipartite (or two-layer) if its set of vertices $V$ is partitioned into two sub sets $V^{1}$ and $V^{2}$, such that $V^{1} \cap V^{2}=\emptyset$ and $E \subseteq V^{1} \times V^{2}$, where each $V^{j}$, $j \in\{1,2\}$ is called a layer. Bipartite networks are also employed in modeling quadratic and 
nonsymmetric/unsymmetric matrices, implying in $\left|V^{1}\right|=\left|V^{2}\right|$ (GEBREMEDHIN; MANNE; POTHEN, 2002a; GEBREMEDHIN; MANNE; POTHEN, 2002b). Let $n=|V|$ and $m=|E|$ denote, respectively, the number of vertices and the number of edges. An edge $(u, v)$ may have an associated weight, denoted as $\omega(u, v)$ with $\omega: V \times V \rightarrow \mathbb{R}^{*}$; a vertex $u$ may have an associated weight denoted as $\sigma(u)$ with $\sigma: V \rightarrow \mathbb{R}^{*}$.

The degree of a vertex $u \in V$, denoted $\kappa_{u}$, is defined as the total weight of its adjacent edges, i.e. $\kappa_{u}=\sum_{v \in V} w(u, v)$. The $h$-hop neighborhood of $u$, denoted $\Gamma_{h}(u)$, is formally defined as the vertices in set $\Gamma_{h}(u)=\{v \mid$ there is a path of length $h$ between $u$ and $v\}$. Thus, the 1-hop neighborhood of $u, \Gamma_{1}(u)$, is the set of vertices adjacent to $u$; the 2-hop neighborhood, $\Gamma_{2}(u)$, is the set of vertices 2-hops away from $u$, and so forth.

A similarity score $S(u, v)$ can be computed from a pair of vertices $u$ and $v$, yielding values in the range $[0,1] \subset \mathbb{R}$, from lowest (0) to highest (1) similarity. Two possible similarity scores are defined in Eqs. 6.1 and 6.2, known as common neighbors and weighted common neighbors (LÜ; ZHOU, 2010), respectively.

$$
\begin{gathered}
S_{c n}(u, v)=|\Lambda(u, v)|, \Lambda(u, v)=\left\{\Gamma_{1}(u) \cap \Gamma_{1}(v)\right\} \\
S_{w c n}(u, v)=\sum_{z \in \Lambda(u, v)} \frac{\omega(u, z)+\omega(v, z)}{\log (1+s(z))} \\
s(z)=\sum_{u \in \Gamma_{1}(z)} \omega(z, u)
\end{gathered}
$$

\subsubsection{Multilevel method in bipartite networks}

Multilevel optimization allows executing complex algorithms on large-scale networks by reducing the size of the network on which the algorithm is to be applied. The strategy comprises three phases, namely coarsening, solution finding with the target algorithm and uncoarsening (VALEJO et al., 2014b; VALEJO; VALVERDE-REBAZA; LOPES, 2014b). Let us consider a problem defined in a bipartite network $G_{0}\left(V_{0}, E_{0}, \omega_{0}\right)$, on which executing a target algorithm is prohibitive. Figure 76 illustrates a multilevel method applied to $G_{0}$, where $G_{L}$ denotes its coarsest version obtained after $L$ coarsening steps (levels); $S_{L}$ denotes the initial solution obtained executing the target algorithm in $G_{L}$; and $S_{0}$ denotes the final solution obtained in the original problem instance $G_{0}$, after executing $L$ refinement steps.

The coarsening phase constructs a hierarchy of coarsened bipartite networks $G_{l}$ from $G_{0}$, $l \in[1, \ldots, L-1]$, yielding intermediate approximations of the original network at multiple levelsof-detail. Coarsening requires two algorithms, namely matching, that defines which vertices will be collapsed, and contraction, which builds the reduced representation once the matching has been defined. A matching $M=\left\{V_{k}\right\}_{k=0}^{K}$ is a partition of $V$ into $K$ non-empty and disjoint subsets $V_{i} \in V$ such that $V=\bigcup V_{i}, 1 \leq\left|V_{i}\right|, \forall i$ and $i \neq j \Rightarrow V_{i} \cap V_{j}=\varnothing$. Restrictions specify 
how vertices can be matched, e.g. a restriction such as $\left|V_{i}\right|=2, \forall i$ implies that vertices must be paired. A vertex $u \in V_{i}$ is said to be matched, otherwise, if $\nexists V_{i} \in M \mid u \in V_{i}$ the vertex is said to be unmatched, or a singleton. An input parameter $\rho \in[0,1] \subset \mathbb{R}$ determines the (maximum) network reduction factor and therefore the cardinality of the matching $M$, denoted as $\zeta$, where $|M|=\left|\cup V_{i}\right|=\zeta=\sum\left|V_{i}\right|$.

For instance, if vertices are to be matched pairwise, then $\zeta=\lceil|V| \rho\rceil,(\rho \in(0,0.5])$. Considering, as an illustration, a network with $|V|=100$ vertices and $\rho=0.3$, then $\sum\left|V_{i}\right|=60$ vertices (or 30 vertex pairs) can will be selected for matching. If $\rho=0.5$, at each iteration the vertex set will be reduced by (at most) a factor of two, yielding a logarithmic decrease on the size of the networks along the multiple coarsening iterations.

The contraction algorithm constructs a coarser network by collapsing a set of matched vertices $\left\{u_{1}, \ldots, u_{n}\right\}$ into a single super-vertex $s V_{i}$. Vertices $\left\{u_{1}, \ldots, u_{n}\right\} \in V_{l}$ that compose a super-vertex $s V_{i} \in V_{l+1}$ are called predecessor-vertices of $s V_{i}$. A successor-network $G_{l+1}$ will inherit the non-matched vertices from its predecessor-network $G_{l}$. In order for $G_{l+1}$ to be a good proxy to its predecessor-network, the weight $\sigma\left(s V_{i}\right)$ of a super-vertex $s V_{i}=\left\{u_{1}, \ldots, u_{n}\right\} \in V_{l+1}$ is computed as the sum of weights of its predecessor-vertices. Furthermore, any edges incident to vertices $\left\{u_{1}, \ldots, u_{n}\right\} \in V_{l}$ are collapsed to obtain the so-called super-edges incident to $s V_{i}$.

coarsening \{matching, contraction\} uncoarsening \{projection, refinement\}

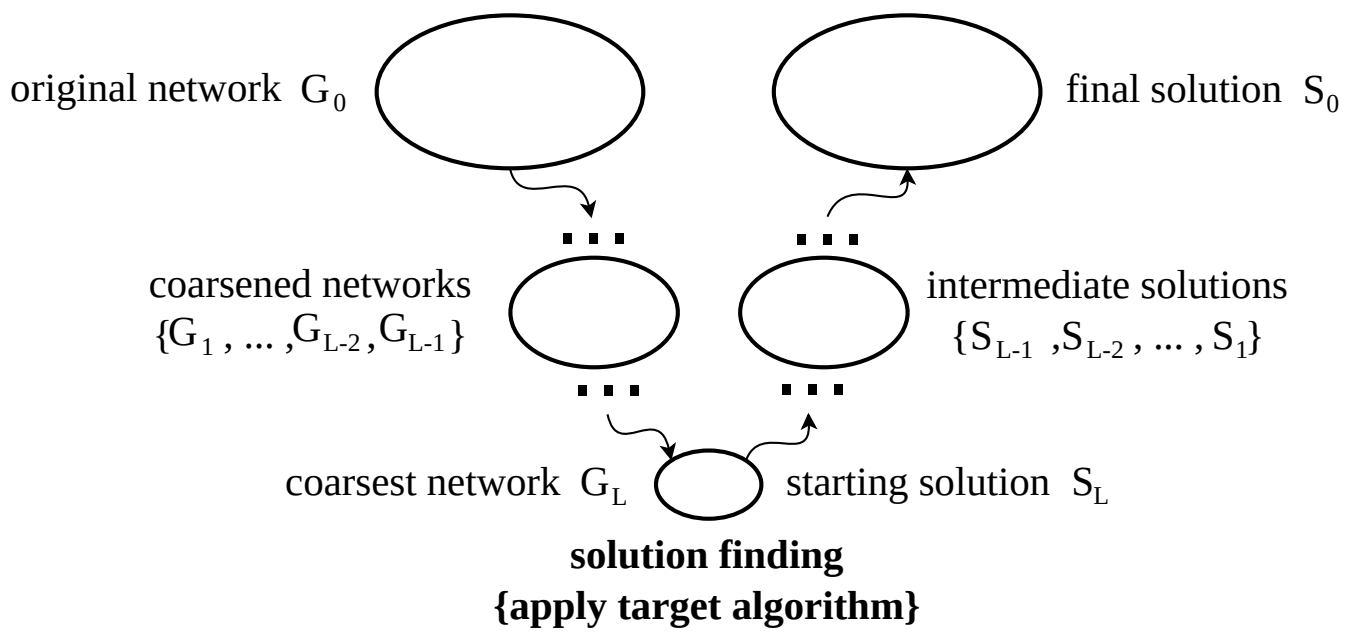

Figure 76 - Phases of a multilevel optimization process executed on input network $G_{0}$ : coarsening, solution finding, and uncoarsening, with the the entailed sequence of intermediate networks and solutions, and final solution $S_{0}$.

Source: Elaborated by the author.

The solution finding phase applies the target algorithm to compute a solution in the coarsest bipartite network $G_{L}$. Let $S$ be the set of all possible solutions in the coarsest problem instance. Given an objective function $f: S \rightarrow \mathbb{R}$ (or $\mathbb{N}$ ) that assigns a cost to each solution in $S$, the aim is to find a state $s \in S$ with optimum cost. For instance, in the traveling salesman problem $f(s)$ expresses the length of a tour $s$, whereas in a community detection problem it denotes some 
measure of community quality.

Finally, the uncoarsening phase, also known as solution projection, successively transfers the solution available at a current level to the next level in the hierarchy. The solution obtained in the coarsest network $G_{L}$ is successively projected through intermediate bipartite networks $G_{L-1}, G_{L-2}, \ldots, G_{1}$ up to the initial network $G_{0}$. A refinement heuristics can be applied to avoid local minima and improve solution quality.

\subsubsection{Coarsening strategies and their limitations}

At any level, a coarsened network must preserve the major topological features of its predecessor-network, i.e., connections and weights of vertices and edges must reflect the corresponding global patterns of their predecessors. However, matching involves many choices and selecting a matching strategy that preserves the structural properties relevant to the problem at hand is a critical component of an effective multilevel implementation. An inadequate strategy may degrade solution quality and increase its computational cost.

Several matching algorithms have been introduced for unipartite networks. Two popular ones, which visit vertices in random order and match vertices pairwise, are Random Matching $(R M)$ and Heavy-Edge Matching (HEM) (KARYPIS; KUMAR, 1998a). In RM, once an unmatched vertex $u$ is visited, one of its unmatched neighbors $v$, if any, will be arbitrarily selected as the match. In $H E M$, a random vertex $u$ is matched with the adjacent vertex $v$ so that edge $(u, v)$ has maximum weight over all adjacent edges to $u$.

These algorithms can not be directly applied to bipartite networks, as they do not distinguish between vertex types and therefore can not enforce a matching that preserves the bipartite property that adjacent vertices are in different layers. However, a bipartite network $G=\left\{V^{1} \cup V^{2}, E\right\}$ can be decomposed into two unipartite networks $G^{1}$ and $G^{2}$, one for each layer, so that adjacent vertices in the bipartite network are made adjacent in their corresponding projection (ZWEIG; KAUFMANN, 2011; BANERJEE; JENAMANI; PRATIHAR, 2017b). This enables executing algorithms such as $R M$ and HEM in each one-mode projection, and the matchings thus obtained can be mapped back to the original bipartite network. Valejo et al. (2017) and Valejo et al. (2018a) rely on this strategy in a matching algorithm for bipartite networks called $O P M$, illustrated in Figure 77.

Figure 77a shows the input bipartite network and the corresponding one-mode projections of each layer are in Figure 77b. A possible outcome from applying the $R M$ algorithm to $G_{1}$ and $G_{2}$ is the matching $M=\left\{\left\{u_{1}, u_{2}\right\},\left\{u_{3}, u_{4}\right\},\left\{u_{5}, u_{6}\right\}\right\}$, also indicated in Figure 77b. $M$ is then transferred to the original bipartite network, Figure $77 \mathrm{c}$. Finally, the contraction algorithm creates the coarsened bipartite network, shown in Figure 77d.

Valejo et al. (2018b) later introduced two novel algorithms designed specifically for matching vertices in bipartite networks, called $R G M b$ and $G M b$. They consider a local 2-hop 
neighborhood restriction which imposes that adjacent vertices can not be matched and vertices can only be matched with others in their 2-hop neighborhood set. This restriction reduces the local search space and the computational cost of finding a match. The $R G M b$ matching is illustrated in Figure 78.

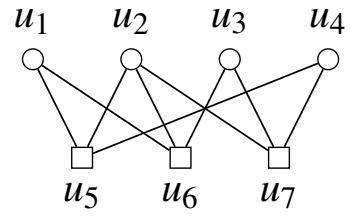

(a) Original bipartite network

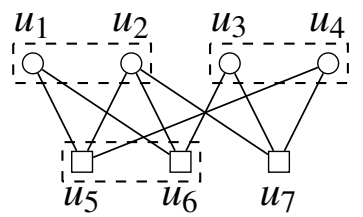

(c) Projected matching
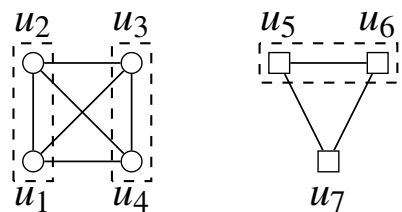

(b) One-mode projections $G^{1}$ and $G^{2}$

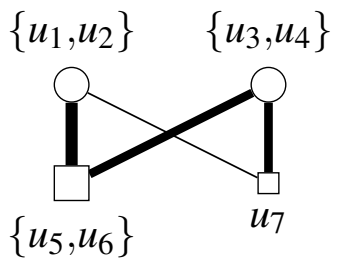

(d) Coarsened network

Figure $77-O P M$ matching of a bipartite network resulting from matching its two one-mode projections with the $R M$ matching algorithm.

Source: Elaborated by the author.

In an initial iteration, depicted in Figure 78a, a selected vertex $u_{1}$ is allowed to match non-adjacent vertices $u_{2}$ or $u_{4}$, which belong to the 2-hop neighborhood of $u_{1}$. Let us assume the pair $\left\{u_{1}, u_{2}\right\}$ is matched. In the next iteration, illustrated in Figure $78 \mathrm{~b}$, a selected vertex $u_{3}$ could match vertices $u_{2}$ or $u_{4}$. Since $u_{2}$ has already been matched, the remaining choice is to match pair $u_{3}$ and $u_{4}$. In the final iteration, the only choice left for the selected vertex $u_{6}$ is $u_{7}$, Figure 78c. Finally, the contracting algorithm creates the coarsened bipartite network, Figure 78d.

Albeit algorithms $O P M, R G M b$, and $G M b$ have been proved useful in several scenarios (VALEJO et al., 2017; VALEJO et al., 2018a; VALEJO et al., 2018b), strategies based on collapsing vertex pairs are inherently limited. For a start, there is an upper limit of 50\% $(\rho=0.5)$ in the rate of network reduction at each iteration. Therefore, coarsening large networks with a high reduction factor demands many coarsening iterations, incurring in high processing and memory costs. Furthermore, vertices considered for matching at early stages are more likely to find good candidate matches that satisfy the similarity function than those considered later in the process. As a result, matching quality tends to degrade towards the final iterations, possibly introducing inconsistencies that will be propagated to upper coarsening levels. In networks with community structures, for instance, vertices from distinct communities may be matched, fragmenting the original topological structure. $k$-cliques are another relevant topological pattern that may be gradually degraded by low quality matches.

The problem is illustrated in Figure 79 on a network with two communities and two 


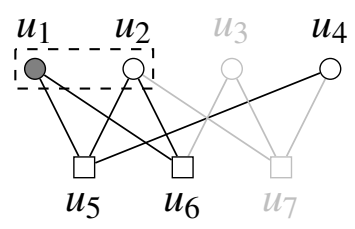

(a)

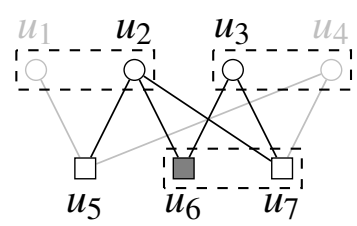

(c)

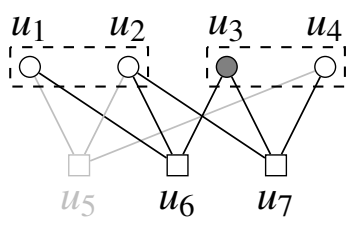

(b)

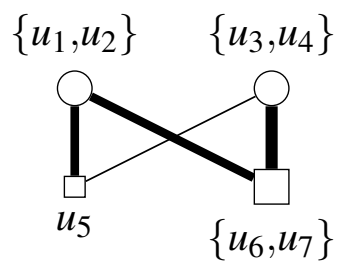

(d) Coarsened network

Figure $78-R G M b$ matching for bipartite networks based on 2-hop neighborhoods.

Source: Elaborated by the author.

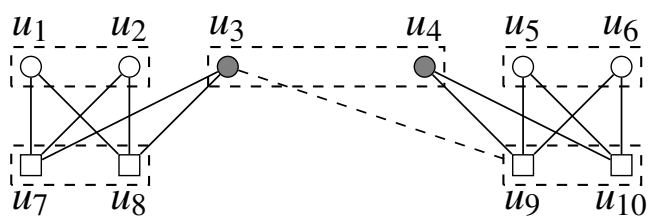

Figure 79 - Matching pair $\left\{u_{3}, u_{4}\right\}$ is a poor choice, as the resulting super-vertex will have predecessor vertices from distinct communities or cliques.

Source: Elaborated by the author.

bicliques. Whereas suitable matching choices have been made in earlier iterations, e.g. pairs $\left\{u_{1}, u_{2}\right\}$ or $\left\{u_{5}, u_{6}\right\}$, in the final iteration the single choice for the algorithm is to match pair $\left\{u_{3}, u_{4}\right\}$. This choice will, at the next coarsening level, degrade the biclique and community structures, since it yields a super-vertex that includes vertices from distinct communities and bicliques.

An additional issue is that super-vertices obtained with pairwise matching tend to be highly unbalanced. At each coarsening level, heavier super-vertices are likely to collapse with their lighter neighboring vertices and super-vertices, so they become even heavier and given higher matching priority in subsequent coarsening levels. Figure 80 illustrates the problem, in which vertex pair $\left\{u_{1}, u_{2}\right\}$ is given priority over pair $\left\{u_{2}, u_{3}\right\}$, even though the number of common neighbors is the same for both pairs. In some applications it may be useful to constrain the size of the super-vertices, or to ensure their are balanced regarding some property. For instance, in solving a document clustering problem it may be desirable to constrain super-vertex (cluster) size. Nonetheless, existing matching algorithms do not support such requirements.

Furthermore, existing matching algorithms require the number of coarsening levels as an input parameter. However, how many levels are necessary to attain a desired network reduction 


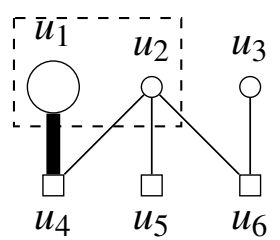

Figure 80 - The priority choice $\left\{u_{1}, u_{2}\right\}$ over the alternative $\left\{u_{1}, u_{3}\right\}$ will produce a highly unbalanced super-vertex.

Source: Elaborated by the author.

factor is typically unknown a priori. From a user perspective, it would be more intuitive to specify the desired size of the coarsest network. In a community detection problem, for instance, this would coincide with the target number of communities; in a visualization problem it would be natural to specify a final network size that fits the screen space, rather than informing how many coarsening levels and which reduction factor.

\subsection{Coarsening via weight-constraint and label propaga- tion}

This section introduces a coarsening strategy (split into matching and contraction algorithms) for bipartite networks that relies on weight-constrained label propagation. The proposed matching and contraction algorithms are introduced and then employed to implement two distinct coarsening variations. Two premises establish the major distinction between current state-of-the-art coarsening algorithms and the ones proposed here:

1. Matchings are not restrict to vertices yet unmatched, i.e. both matched and unmatched vertices are allowed to form a new match.

2. The weights of super-vertices obtained from the matchings can be constrained.

The first premise allows super-vertices to collapse more than two vertices at once, so that vertices may be grouped which have common properties or play similar roles in the network, with multiple advantages. Greater network reduction factors may be attained at each coarsening level, e.g., up to $60 \%-90 \%$ depending on the weight constraints imposed on super-vertices. Therefore, fewer coarsening levels are necessary to attain a desired reduction rate, decreasing memory requirements and execution times. Finally, vertices considered for matching later in the process will not be restricted to a few choices. Overall, this approach yields a more consistent and compact coarsening hierarchy. The second restriction allows imposing an upper limit on the super-vertices weights, which is useful to generate super-vertices compatible with the original network structures and to prevent super-vertex weights deviating significantly from the average. 
As matching algorithms must be fast, a cost effective approach is required to handle the weight constraints. In the proposed algorithm, Eq. 6.3 defines the maximum weight of a super-vertex in layer $j$ and coarsening level $l$ (henceforth the subscribed $l$ is suppressed for simplification):

$$
\begin{gathered}
S_{\max }^{j}=\left\lceil(1+\mu) \frac{\left|V_{0}^{j}\right|}{\zeta}\right\rceil, \\
\zeta=\left\lceil\left|V^{j}\right|(1-\rho)\right\rceil
\end{gathered}
$$

where parameter $\mu \in(0, \zeta] \subset \mathbb{R}$ places an upper bound in the weight constraint, where $\left|V_{0}^{j}\right|$ is the number of vertices in each layer $j \in\{1,2\}$ at the initial coarsening level $l=0$. From Eq. 6.3, $\mu=\zeta$ implies $S_{\text {max }} \approx\left|V_{0}^{j}\right|$, i.e, a super-vertex $s V \in V_{l+1}$ may include all vertices $\in V_{l}$. A vertex $v$ with weight $\sigma(v)$ can be matched with a vertex $u \in s V_{i}$ if Eq. 6.5 is satisfied:

$$
\sigma\left(s V_{i}\right)+\sigma(v) \leq S_{\max }^{j}
$$

A super-vertex $s V_{i}$ is said to be overloaded if $\sigma\left(s V_{i}\right)>S_{\text {max }}$ and underloaded if $\sigma\left(s V_{i}\right) \leq$ $S_{\text {max }}^{j}$. Notice that the value $S_{\text {max }}^{j}$ is updated at each coarsening level and depends on $\zeta$, which makes this formulation problem-specific. Figure 81 illustrates how the upper bound parameter $\mu^{j}$ impacts the super-vertex weights $S_{\text {max }}^{j}$ considering one layer $(j=1)$ of a bipartite network with $\left|V^{1}\right|=100,000$ and reduction factor $\rho^{1}=0.5$.

The impact is limited along the initial coarsening levels; in contrast, high values of $\mu$ lead to heavy and probably unbalanced super-vertices in the final levels. In the particular case of $\mu=1.0$ and $\rho=0.5$ one observes a similar behavior to standard pairwise matching algorithms $O P M, G M b$ and $R G M b$. For instance, in the first level $\zeta^{1}=\lceil 100.000 \cdot 0.5\rceil$, then $S_{\text {max }}^{1}=\lceil 1 \cdot 100.000 / 50.000\rceil=2$, i.e., each super-vertex is formed by a pair of vertices, or $\left|V_{i}\right|=$ $2 \forall V_{i} \in M$.

In the light of the previous discussion we introduce a near-linear matching algorithm called matching via weight-constrained label propagation in bipartite networks (MLPb), outlined in Alg. 20.

It incorporates the aforementioned premises and is based on the well-known Label Propagation Algorithm (LPA) for community detection in networks introduced by Raghavan, Albert and Kumara (2007). Because it is simple and fast, LPA provides an appropriate framework for a matching algorithm. However, whilst in its original formulation the algorithm returns a community membership array, $M L P b$ outputs a hierarchy of increasingly simplified networks, which may be considered for solving multiple tasks, community detection included.

Algorithm $M L P b$ takes as inputs the bipartite network at a coarsening level $l, G_{l}=$ $\left(V^{1} \cup V^{2}, E, \sigma, \omega\right)$ and the layer to be coarsened $j=\{1,2\}$. Coarsening may be applied to one or 


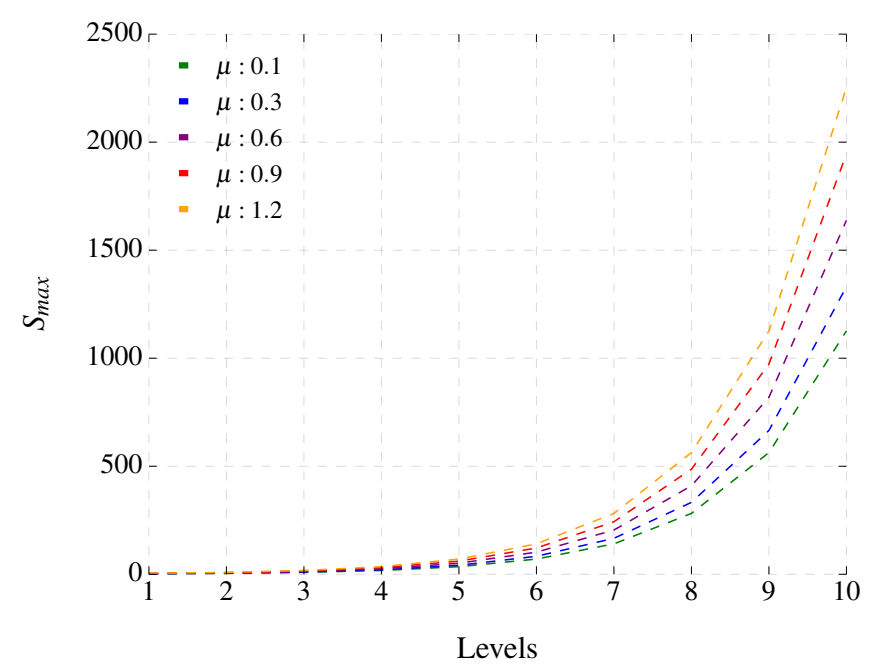

Figure 81 - Impact of the upper bound parameter $\mu$ on the constrained weights of super-vertices $S_{\max }^{1}$ along the coarsening levels.

Source: Elaborated by the author.

both layers as $M L P b$ operates on the layers independently to fit their corresponding parameters $S_{\text {max }}^{j}$ and $\zeta^{j}$. The remaining parameters are (for the corresponding layer to be coarsened) a target reduction factor $\rho=(0,1.0] \subset \mathbb{R}$, an upper bound $\mu \in(0, \zeta] \subset \mathbb{R}$, and two stopping criteria, namely a maximum number of iterations $T \subset \mathbb{Z}_{+}$and a tolerance parameter $\tau \subset \mathbb{R}_{+}$which specifies a minimum threshold on label updates. It returns the matching $M=\left\{V_{k}\right\}_{k=0}^{K}$, which can be stored as a union-find or as a simple array structure. $M(u)$ denotes a function that returns the super-vertex $s V_{i}$ that includes vertex $u$, and $M(u)=s V_{i}$ is an operation that collapses $u$ into the existing super-vertex $s V_{i}$.

$M L P b$ propagates vertex labels throughout the network to create the super-vertices. A single label remains within a group of matched vertices that will collapse into a single supervertex. Furthermore, to ensure independent handling of the layers, vertices can only match others in their set of 2-hop neighbors. This restriction implies, from the definition of bipartite networks, that adjacent vertices are not matched and potential matches of a vertex $u$ can only be found in its own layer.

The algorithm initially computes $S_{\max }$ and each vertex is initialized as a singleton with a unique label (lines 1-2). Vertices are visited in random order (line 5), and a randomly picked vertex $u$ is matched within its 2-hop neighborhood with the vertex for which the number of weighted common neighbours is maximal (lines 7-13). As super-vertices are weight-constrained, a match is eligible if it does not yield an overloaded super-vertex (line 9). The matching process proceeds updating vertex labels and will be interrupted once the specified minimum number of vertices $\zeta$ is attained (line 18). Alternatively, the stopping conditions are given by input parameters $T$ (the maximum number of iterations) and $\tau$ (a tolerance threshold on label updates): the current execution stops if either $T$ has been reached or the number of label updates is smaller 


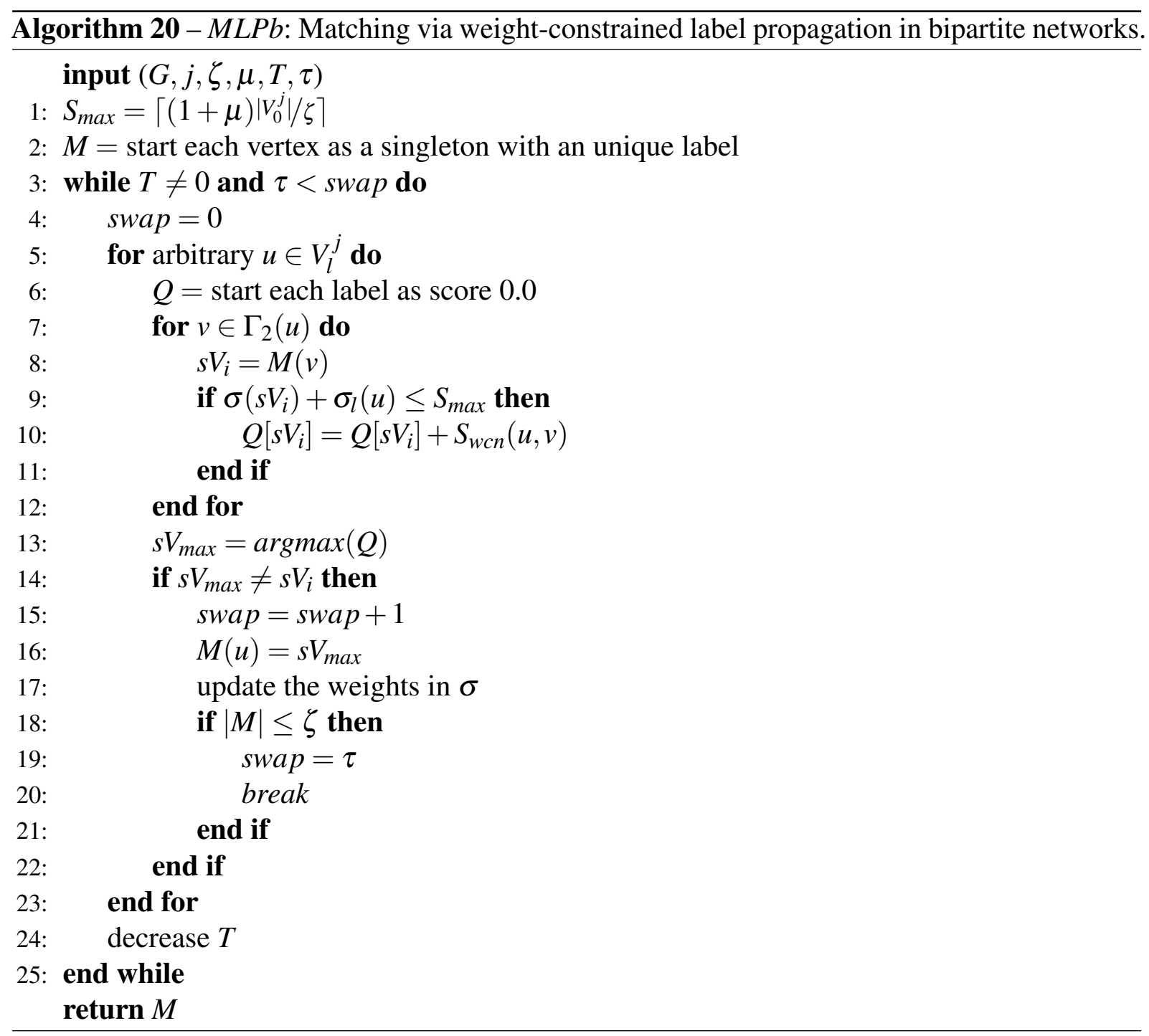

than or equal to tolerance $\tau$.

The contraction algorithm is described in Alg. 21, and takes as input a matching $M$ where matches are given by groups of vertices rather than vertex pairs. Groups of matched vertices are mapped into a successor vector and collapsed into a single super-vertex $s V_{i} \in G_{l+1}$ (lines 3-7). The successor vector will be accessed again in the uncoarsening phase to project the solution relative to its super-vertex $s V_{i}$. Any vertices not included in $M$ are inherited by $G_{l+1}$ (line 10). After the mapping, adjacent edges in $E_{l}$ are collapsed into a single super-edge and added to $E_{l+1}$ (lines 11-18), i.e. each pair $(u, v) \in E_{l}$ entails a successor $(w, z)$. If the successor is already in $E_{l+1}$, its weight is increased by $\omega_{l}(u, v)$; otherwise, a new edge $(w, z)$ with weight $\omega_{l}(u, v)$ is inserted into $E_{l+1}$ (lines 15-18).

A network coarsened with $M L P b$ is, by definition, weighted, i.e., even if the initial network is unweighted the super-edges created in the coarsening will be assigned their edge count as weights. If not relevant for solving the target problem edge weights may be simply ignored. 


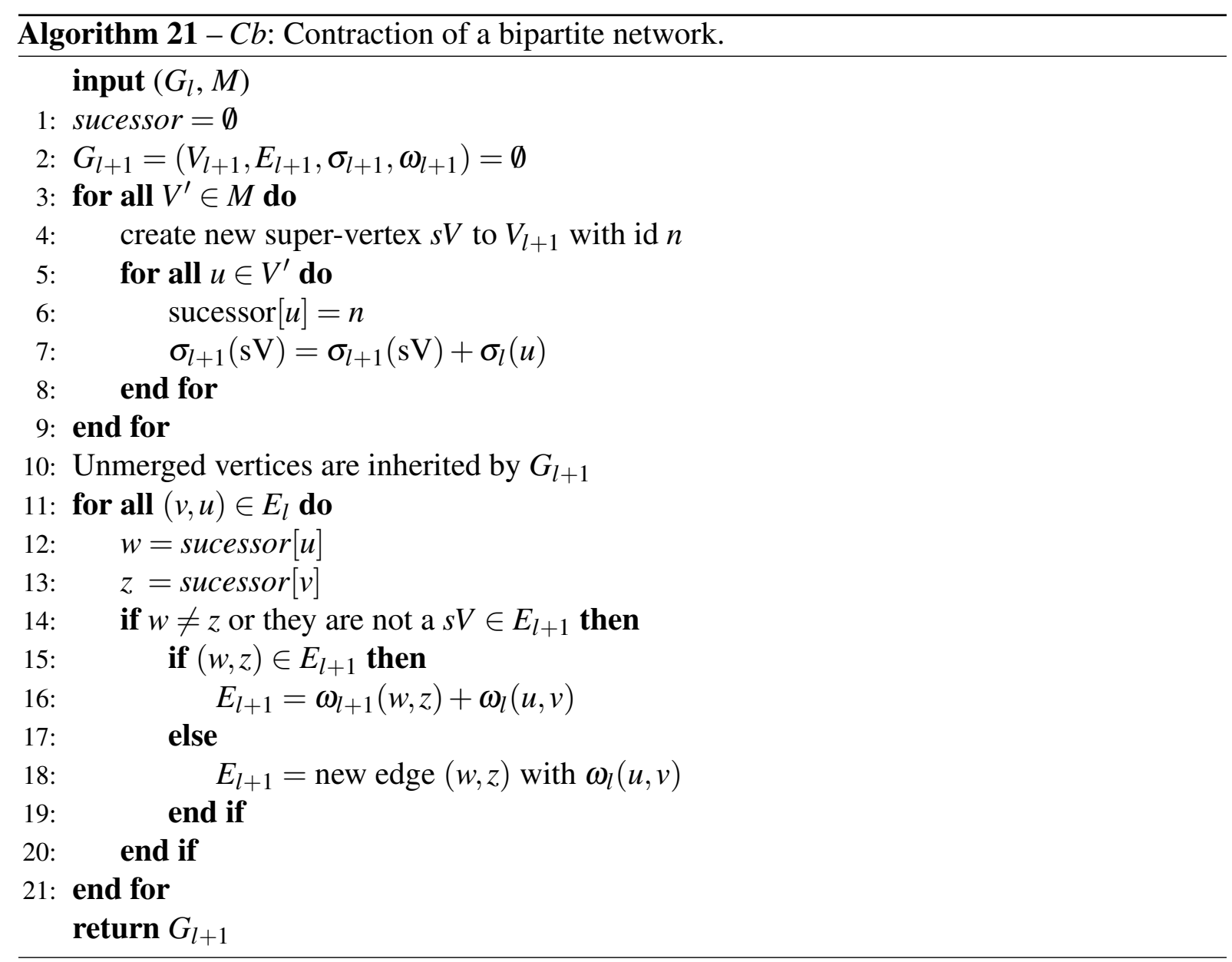

Finally, algorithms $M L P b$ and $C b$ can be integrated into a coarsening strategy. We propose two variations that differ in how the coarsening parameters are specified. Algorithm $M L P b_{C S L}$ implements a standard formulation of the multilevel method, where the user directly sets the desired number of coarsening levels $L$ and a reduction factor $\rho$. In contrast, in algorithm $M L P b_{C S V}$ the user input is the desired size (number of vertices) expected in the coarsest network, $\zeta$, and it builds the coarsening hierarchy so as to obtain a coarsest network with the user-specified size.

Alg. 22 describes $M L P b_{C S L}$. The inputs are, for each layer, the maximum number of levels $L=\left\{L^{1}, L^{2}\right\}$, the reduction factor $\rho=\left\{\rho^{1}, \rho^{2}\right\}$, the upper bound on super-vertex weights $\mu=\left\{\mu^{1}, \mu^{2}\right\}$ and the stopping conditions $T=\left\{T^{1}, T^{2}\right\}$ and $\tau=\left\{\tau^{1}, \tau^{2}\right\}$.

Each layer is coarsened (line 4), level by level, by means of calls to matching algorithm $M L P b$ (line 6), with $\zeta$ computed as a function of the current number of vertices and the aimed reduction factor (line 5). Finally, algorithm $C b$ (line 10) builds the coarser network from the matching $M$. This process continues until each layer is coarsened at the desired number of levels.

Alternatively, Alg. 23 describes $M L P b_{C S V}$, where input parameters $\zeta=\left\{\zeta^{1}, \zeta^{2}\right\}$ specify the desired size of each layer.

In this case, the value $S_{\max }$ is static and the number of coarsening levels depends on how 

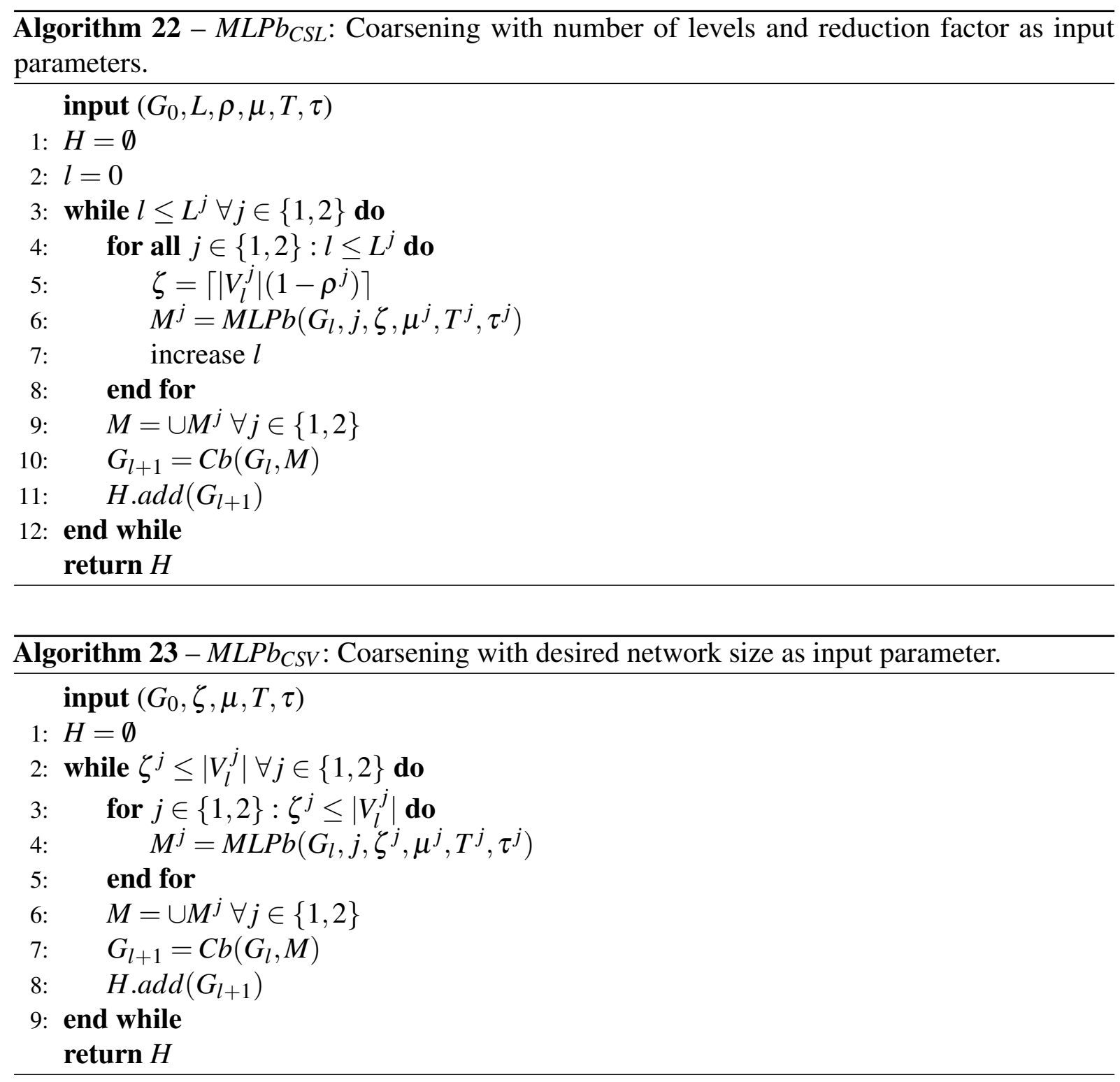

many iterations the algorithm executes before it stops. The algorithm applies the highest possible $\rho$ at each level.

Figure 82 illustrates the behavior of algorithm $M L P b_{C S L}$ on a dense weighted bipartite synthetic network obtained setting $|V|=2,000, c=5$ communities and noise level $n=0.1$. Networks are drawn with a bipartite layout. Coarsening was executed setting $\rho=0.5$ and $L=5$. The initial network in shown in a, whereas b, c, d, e and f show, respectively, the coarsened networks at levels 1 to 5 , with reduction factors of $25 \%, 50 \%, 75 \%, 90 \%$ and $95 \%$.

Similarly to state-of-the-art coarsening strategies, algorithms $M L P b$ are directly applicable in community detection tasks: each super-vertex in the coarsest network, illustrated in Figure $82 \mathrm{f}$, may be interpreted as a community. In projecting the solution back to the original network the predecessor vertices of a super-vertex will be assigned to a community, identified by a corresponding color in Figure 82g. This strategy resembles bi-clustering or co-clustering solutions, which also define communities independently in each layer, unlike standard community 
detection algorithms in which communities obtained are formed by vertices from both layers. This feature may be useful in some contexts, for instance, when just one of the layers must be coarsened, as illustrated in Figure 82f, or the layers must be coarsened at different rates.

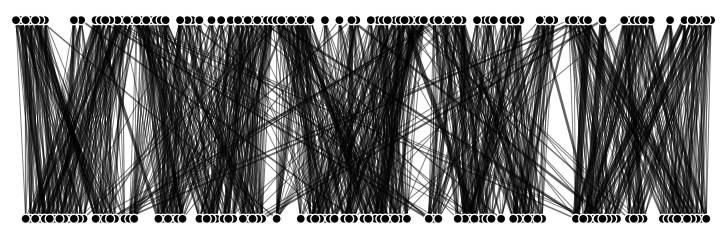

(a)

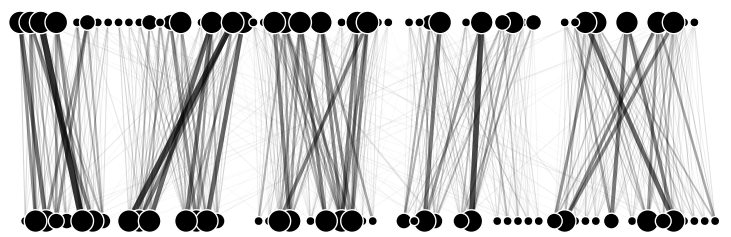

(c)
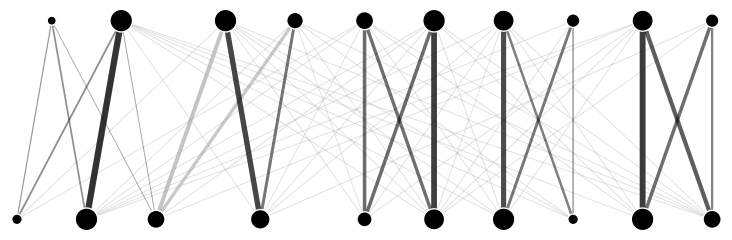

(e)

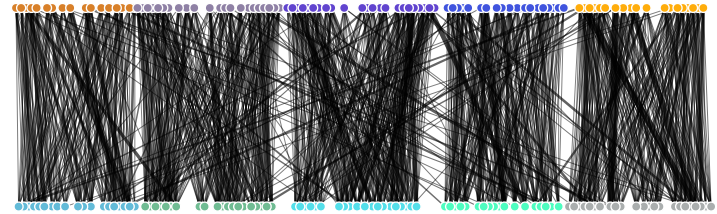

(g)

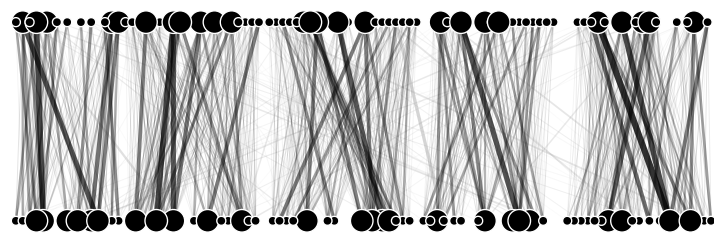

(b)

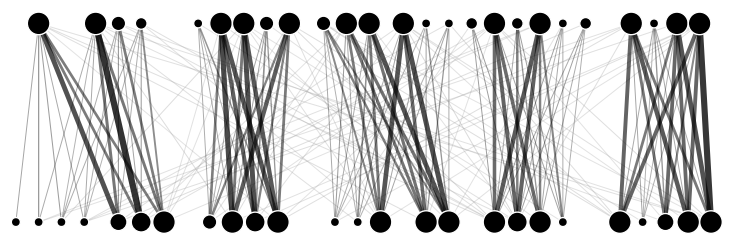

(d)
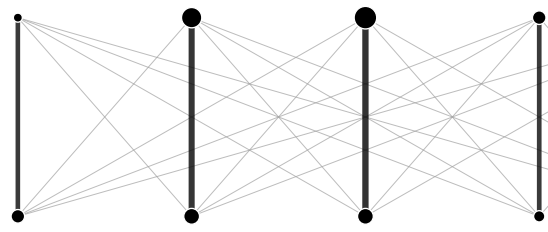

(f)

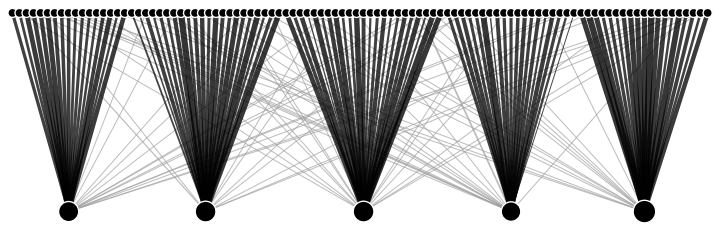

(h)

Figure 82 - A bipartite network coarsened at increasing reduction rates with the $C S V$ algorithm. The original network is shown in (a) and the networks at reduction rates of $25 \%, 50 \%, 75 \%, 90 \%$ and 95\% are shown, respectively, in (b), (c), (d), (e) and (f). Considering the super-vertices in $\mathrm{f}$ as communities and projecting them back to the original network, the result is summarized in $(\mathrm{g})$, in which colors indicate the originating super-vertex. Finally, the plot in (h) shows the result of coarsening just the second layer to five super-vertices.

Source: Elaborated by the author.

\subsection{Parametric and complexity analysis}

The $M L P b_{C S V}$ implementation (Alg. 23) is more intuitive to use, as it suppresses input parameters $L$ and $\rho$, replacing them with $\zeta$, the target network size, which is more intuitive to specify. We conducted a parametric analysis to assess its convergence behavior. Analysis was conducted on synthetic networks of varying degree distributions and clustering coefficients, obtained employing a community generation model that mimics properties of real-world networks regarding noise, community structure and overlapping (VALEJO et al., 2019). 
We considered a fixed value of parameter $\zeta$ for both layers, so the algorithm will iterate until $\zeta^{1}=\zeta^{2}=10$, and created networks with the following settings of the generating model: vertex set sizes $|V|=\left|V^{1}\right|+\left|V^{2}\right|$ varying in the range [1,000,100,000] at increments of 300; noise levels $n$ varying randomly in the range [0.1,0.4]; numbers of communities given by $c=0.01|V|$; network density $d$ varying in the range $[0.1,0.5]$. Overall, in the following analyses we considered 100 distinct configurations and replicated each configuration ten times to yield 1,000 synthetic networks.

Figure 83 shows the points depicting how many coarsening levels $L$ have been created before the algorithm stops, as a function of network size $|V|$. Notice that, in general, $L$ increases slowly as $|V|$ increases. In summary, on smaller networks convergence occurs with $L$ in the range $[2,6]$, whereas on larger networks it occurs with $L$ in the range $[6,10]$.

Figure 84 depicts how many iterations have been executed before the algorithm stops, also as a function of $|V|$. Each line refers to a particular setting of the tolerance parameter $\tau$. In general, $T<10$ iterations are required regardless of the value of $\tau$. $T$ increases quickly for network sizes up to 40,000 vertices, slowly for network sizes in the range $[40,000-80,000]$ vertices, and remains stable on larger networks. Higher values of $\tau$ speed up convergence.

Despite the simplification introduced by replacing input parameters $L$ and $\rho$ with parameter $\zeta$, the need of setting the stopping conditions $T$ and $\tau$ may be seen as a potential drawback of the $M L P b$ matching algorithms. Both are standard parameters of label propagation strategies and strategies are often employed to suppress them (XIAOJIN; ZOUBIN, 2002; RAGHAVAN; ALBERT; KUMARA, 2007; ZHOUA et al., 2016). In the case of coarsening, one may define (i) the number of iterations $T$ in terms of a percentage of the number of vertices matched in the first iteration; (ii) the tolerance parameter as equal to one, i.e. $\tau=1$; and (iii) the upper bound on super-vertex weights as equal to the minimum size of layer $j$, i.e. $\mu^{j}=\zeta^{j}$. These are ad hoc values based on our experience with application of the method.

We also analyze the computational cost of coarsening considering the time complexity of both $M L P b$ (Alg. 20) and $C b . M L P b$ is executed $T$ times and requires few iterations to converge, hence we consider $T$ as a constant factor. The loop in line 7 iterates over $\left|V^{j}\right|-\zeta$, hence, in general, it takes time $\varphi=\left|V^{j}\right| / 2$ with $\varphi \ll|V|$. The algorithm finds the $h$-hop neighborhood $\Gamma_{h}(u)$ of each selected vertex $u$ (line 10) at a cost $\mathscr{O}(n\langle\kappa\rangle h)$, where $\langle\kappa\rangle$ is the average network degree. In sparse networks $\langle\kappa\rangle \ll n$ and $m \approx n$. Restricting $h=2$ yields notable computational savings, since $\Gamma_{2}$ can be reached in near-linear time. Therefore, in the average case, the time complexity is $\mathscr{O}(T \varphi\langle\kappa\rangle)$. In the worst-case, i.e., dense networks with high $\langle\kappa\rangle$ and balanced layers, the time complexity is $\mathscr{O}\left(\left|V^{j}\right|^{2}\right)$ with $\left|V^{j}\right| \approx|V| / 2$. In the best-case, i.e., sparse networks with small $\langle\kappa\rangle$ and a small reduction factor the cost is $\mathscr{O}(\varphi)$ with $\varphi \ll|V| / 2$.

The contraction Algorithm $C b$ (Alg. 21) first iterates over all collapsed vertices in $V^{\prime}=\cup V_{i} \in M$ in order to create the sucessor data structure and the super-vertices. Next, each edge in $E_{l}$ is selected to create the super-edges. Therefore, in the worst-case, i.e. $\left|V^{\prime}\right|=|V|$, 


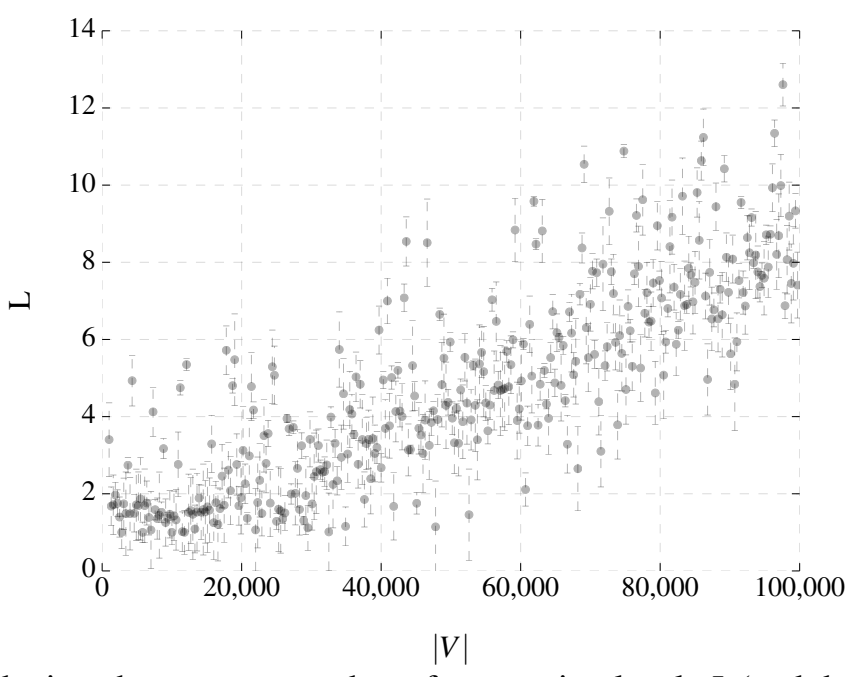

Figure 83 - Each point depicts the average number of coarsening levels $L$ (and the corresponding standard deviation errors) required for $M L P b$ to converge (or stop), as a function of the network size $|V|=\left|V^{1}\right|+\left|V^{2}\right|$.

Source: Elaborated by the author.

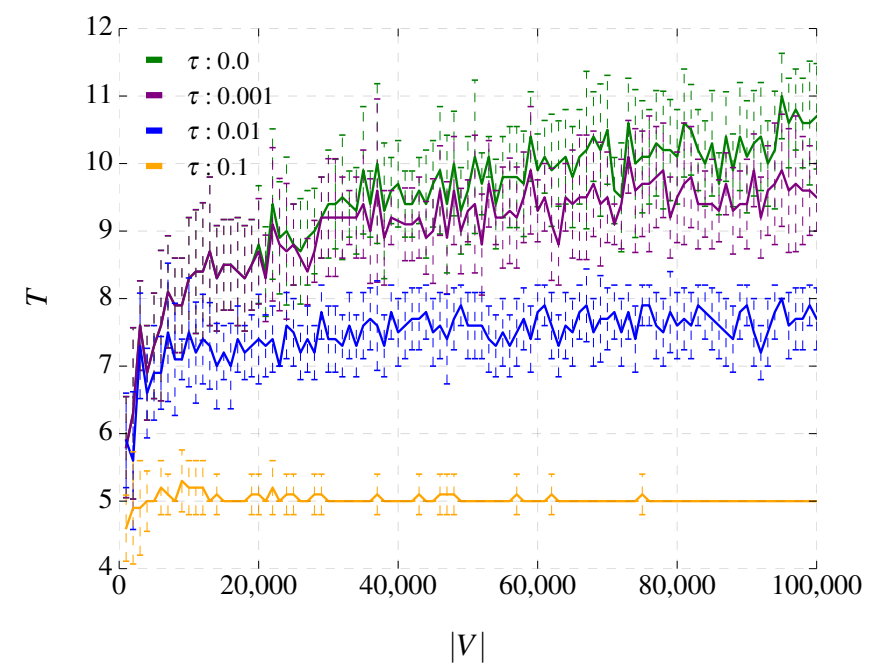

Figure 84 - Number of iterations $T$ required for $M L P b$ to converge (or stop) as a function of the network size $|V|$. Each line corresponds to a choice of the tolerance parameter $\tau$.

Source: Elaborated by the author. 
the asymptotic complexity is $\mathscr{O}(|V|+|E|)$. In the best-case, i.e. a sparse network and a small reduction factor or small $\zeta$, it runs in $\mathscr{O}\left(\left|V^{\prime}\right|+|E|\right)$ with $\left|V^{\prime}\right| \ll|V|$ and $|E| \approx|V|$, then complexity is $\mathscr{O}(|V|)$.

\subsection{Experimental Results and Analysis}

We conducted three experimental studies to assess the proposed coarsening algorithms:

1. A first study compared the performance of $M L P b_{C S L}$ with three state-of-the-art coarsening algorithms to optimize an expensive community detection algorithm. The goal is to evaluate the quality the topological structure of the coarsened representations obtained.

2. A second study employed $M L P b_{C S V}$ as a community detection algorithm, comparing results with those obtained with three popular and fast community detection algorithms. The goal is two-fold: evaluate to which extent the super-vertices created are representative of the network community structures; and verify the algorithm's flexibility to handle different tasks.

3. Finally, a third study employed $M L P b_{C S V}$ in association with a force-directed network drawing algorithm for purposes of visualizing major topological structures in large bipartite networks. The goal is to assess to which extent the coarsening of the networks can faithfully preserve and summarize the global properties of bipartite networks with distinct topological characteristics.

Experiments were executed on a Linux machine with an 8-core processor with $3.7 \mathrm{GHz}$ CPU and 64GB main memory. All algorithms were implemented in Python with the $\operatorname{Igraph}^{1}$ and Networkx ${ }^{2}$ libraries.

\subsubsection{Benchmark and optimization problem}

Research efforts on the multilevel method were strongly motivated by expensive optimization algorithms, most notable community detection, which established itself as a benchmark problem in this context. Therefore, we assess the proposed coarsening strategy in the context of multilevel community detection on bipartite networks.

Larremore, Clauset and Jacobs (2014) formulated a bipartite stochastic block model algorithm to detect communities in bipartite networks $\left(b i S B M^{3,4}\right)$. BiSBM relies on the KerninghanLin criterion as a heuristic to infer the ideal number of communities, and has competitive

1 available from $<$ http://igraph.org/python/>

2 available from $<$ https://networkx.github.io/ $>$

$3 \mathrm{C}++$ : $<$ http://danlarremore.com/bipartiteSBM/>

4 Python interface: <https://github.com/junipertcy/det_k_bisbm> 
performance relative to state-of-the-art methods. However, it is too expensive to be executed on large-scale networks.

We thus considered $b i S B M$ as the target algorithm for a comparative assessment of the new $M L P b_{C S L}$ coarsening algorithm. The goal is to investigate to which extent the coarsening processes executed with $M L P b_{C S L}$ and with other state-of-the-art coarsening algorithms for bipartite networks can yield solutions statistically equivalent in quality to the standard biSBM, used as baseline, while increasing its scalability. Our experimental study compared $M L P b_{C S L}$ with coarsening algorithms $O P M_{h e m}, R G M b$ and $G M b$, discussed in Section 6.2. We assessed the runtime and quality of the reduced networks obtained from each algorithm. Henceforth, the multilevel versions of $b i S B M$ will be identified as $M L P b_{C S L}+b i S B M, G M b+b i S B M, R G M b+b i S B M$ and $O P M_{h e m}+b i S B M$.

A set of 60 parameter configurations were considered to generate synthetic weighted bipartite networks with distinct community structures (VALEJO et al., 2019), as follows: $|V|=$ 1,000 with $\left|V^{1}\right|=\left|V^{2}\right|, n$ within the range $[0.0025,0.3]$ at increments of 0.0025 , and the number of communities $c=10$. Finally, the network density $d$ varied randomly in the range $[0.1,0.8]$ at increments of 0.1. Each network setting was replicated 10 times, resulting in 600 synthetic networks. Two performance measures were considered: normalized mutual information (NMI) and runtime (seconds). The NMI index quantifies the quality of the disjoint clusters inferred (LABATUT, 2013) and is often employed to compare community detection algorithms.

Figure 85 shows the NMI accuracy values as a function of the amount of noise in the synthetic networks. Figure85a shows results from the baseline and its multilevel version at one level of coarsening (i.e., $L=1$ ) with $\rho=0.5$ (i.e., reducing by half the number of vertices) for all layers. In general terms and considering the variability measured by the standard deviation, the best performances were achieved by the baseline and the multilevel version $M L P b_{C S L}+b i S B M$. However, considering the noise parameter varying in the range in $[0.25,0.3]$, the baseline yielded the best average performance in all networks. NMI values for $G M b+b i S B M, R G M b+b i S B M$ and $O P M_{h e m}+b i S B M$ decrease more quickly than the baseline and $M L P b_{C S L}+b i S B M$, as the noise levels increase. Furthermore, considering the noise in the range $[0.15,0.3], G M b+b i S B M$, $R G M b+b i S B M$ and $O P M_{h e m}+b i S B M$ yielded the lowest average performance in all networks. In this case, the reduced network representations created with $M L P b_{C S L}$ proved more robust than those obtained with $G M b, R G M b$ and $O P M_{\text {hem }}$.

Figure $85 \mathrm{~b}$ shows the results of the baseline and its multilevel versions considering $L=2$ and $\rho=0.5$ for all layers. For noise levels within the range $[0.0025,0.15]$ the baseline and $M L P b_{C S L}+b i S B M$ yielded the best performances. However, for noise levels above 0.15 the baseline yielded the best average performance. In this case, $G M b+b i S B M, R G M b+b i S B M$ and $O P M_{h e m}+b i S B M$ show similar performance and high sensitivity to the noise level (NMI values decrease fast as noise increases), whereas results from $M L P b_{C S L}+b i S B M$ are closer to the baseline, i.e., the decrease in NMI values is slower compared to other coarsening algorithms, 


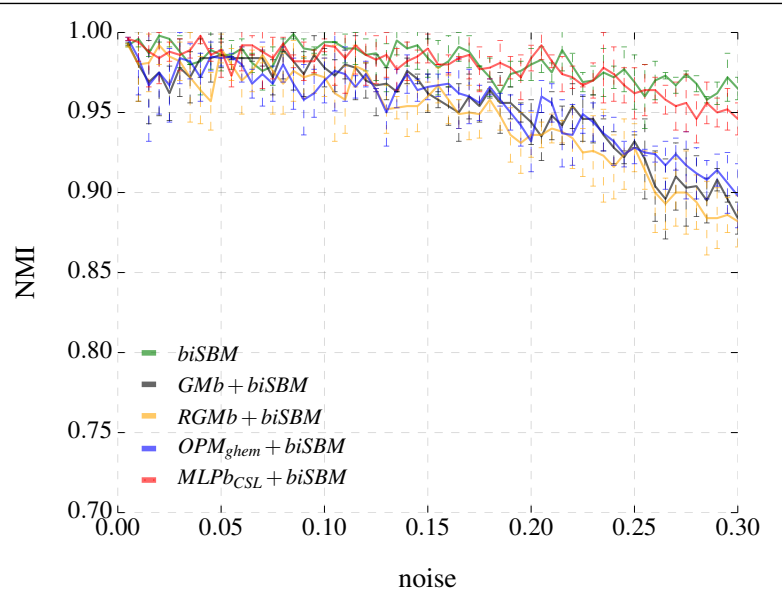

(a)

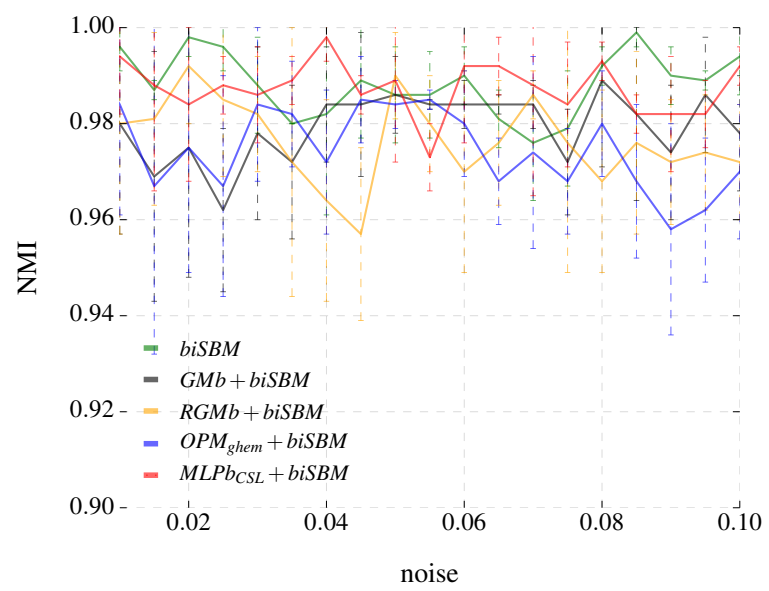

(c)

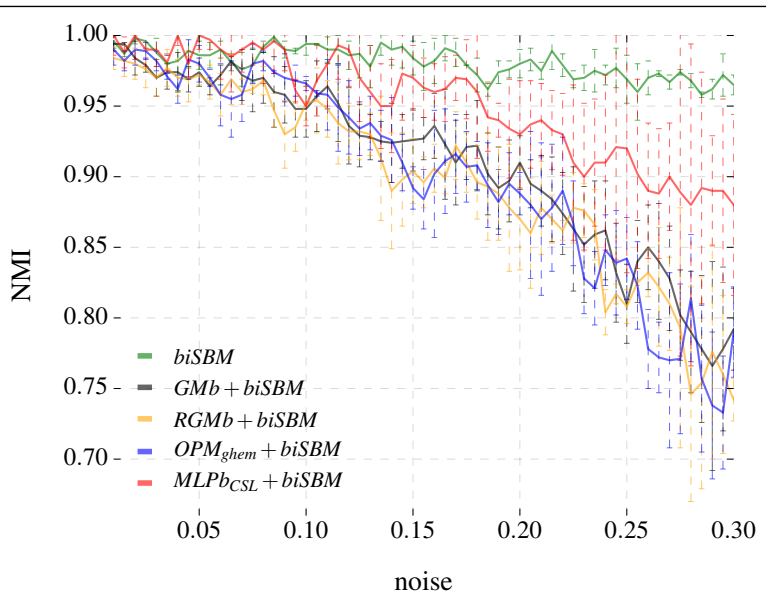

(b)

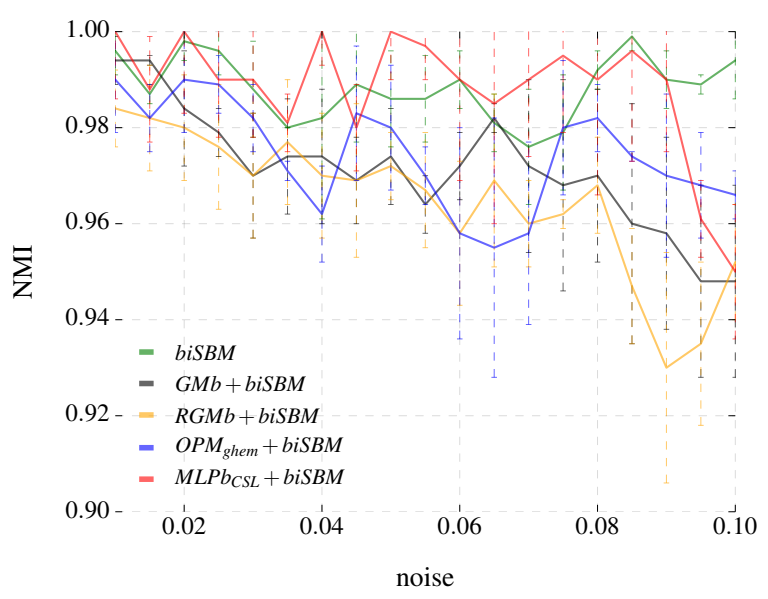

(d)

Figure 85 - NMI accuracy values of biSBM and its multilevel versions as a function of the noise levels for 60 synthetic networks with different properties. (a) NMI values achieved by the baseline and its multilevel method at one level of coarsening; (b) zoomed-in view of the plot for noise levels in the range $[0.0025,0.1]$; (c) NMI values at two levels of coarsening; (d) zoomed-in view of the plot for noise levels in the range $[0.0025,0.1]$.

Source: Elaborated by the author.

suggesting greater robustness to noise. Furthermore, with $L=2$ results from all coarsening algorithms exhibit high standard deviations, suggesting instability. This might be explained by the sharp decrease in the number of potential super-vertices at each coarsening level.

Figures $85 \mathrm{c}$ and $85 \mathrm{~d}$ illustrate the zoomed-in plots focusing on networks with low noise levels, in the range $[0.0025,0.1]$. One notices a small loss in accuracy, even at two levels of coarsening. Moreover, the standard deviations of the values suggest the difference between the baseline and the multilevel versions is not significant.

A Nemenyi post-hoc test (DEMSAR, 2006) was applied to the results in Figure 85 to verify statistical significance of the observed differences in algorithm performance. Results are shown in Figure 86, in which 86a and 86b depict, respectively, the ranks for $L=1$ and $L=2$, and $86 \mathrm{c}$ and $86 \mathrm{~d}$ depict the average ranks relative to the zoomed-in plots. The critical difference 
(CD) is indicated at the top of each diagram and algorithms' average ranks are placed in the horizontal axes, with the best ranked to the left. A black line connects algorithms for which there is no significant performance difference. According to the Nemenyi statistics the critical value for comparing the average-ranking of two algorithms at the 95 percentile is 0.97 for $86 \mathrm{a}, 0.99$ for $86 \mathrm{~b}, 1.73$ for $86 \mathrm{c}$ and 1,76 for $86 \mathrm{~d}$.

Let us consider the outcome of the post-hoc test for $L=1$, i.e., when the number of vertices $n$ has been reduced by a factor of two, shown in Figure 86a. Baseline biSBM ranked best, followed by $M L P b+b i S B M$ and then the group $G M b+b i S B M, R G M b+b i S B M$ and $O P M_{h e m}+b i S B M$. Furthermore, even though it performs better than the other algorithms, performance differences between $b i S B M$ and $M L P b_{C S L}+b i S B M$ are not statistically significant. Similarly, for $L=2$ (Figure $86 a$ ), biSBM ranked first, with no statistically significant difference observed relative to $M L P b_{C S L}+b i S B M$.

Interestingly, in the networks with noise levels in the range $[0.0025,0.1]$, for both $L=1$ and $L=2$, algorithm $M L P b_{C S L}+b i S B M$ ranked best, reinforcing that $M L P b_{C S L}$ can filter the solution space while preserving important topological features. For $L=2$ both $M L P b_{C S L}+b i S B M$ and $b i S B M$ ranked first. In contrast, the accuracy of $G M b, R G M b+b i S B M$ and $O P M_{h e m}+b i S B M$ deteriorates, mainly when $L=2$, indicating that coarsening at this level does not necessarily preserve the original topological properties and is likely to blur community boundaries. The impact of the choice of $L$ depends on network size, i.e., variations in algorithm accuracy are likely to decrease as network sizes increase, hinting that adopting more aggressive coarsening factors might be a successful strategy when handling larger networks.

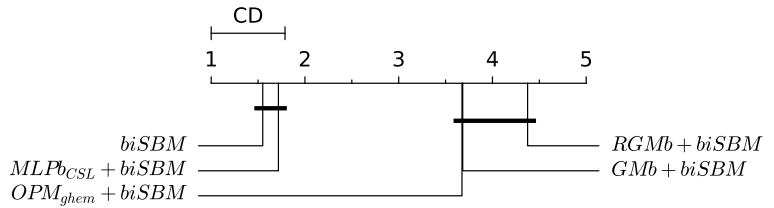

(a)

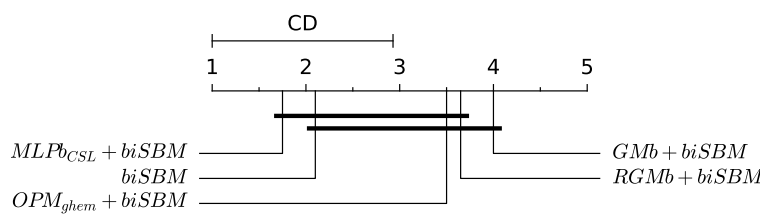

(c)

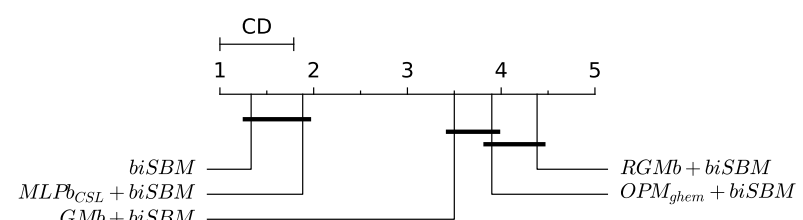

(b)

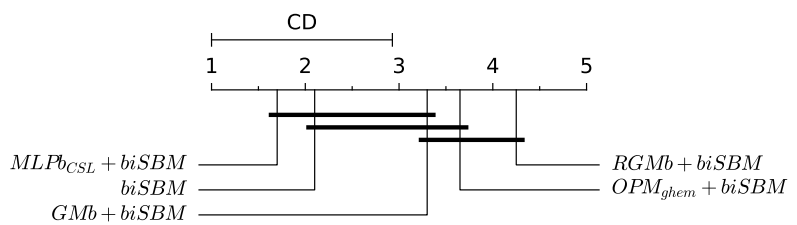

(d)

Figure 86 - Nemenyi post-hoc test for biSBM and its multilevel versions: a shows rank values achieved by the baseline and its multilevel method with one level of coarsening and (c) illustrates the rank values for the zoomed-in plot with noise in the range in $[0.0025,0.1]$; (b) shows the rank values with two levels of coarsening and (d) illustrates the rank values for the zoomed-in plot with noise in the range of $[0.0025,0.1]$.

Source: Elaborated by the author.

We also assessed the scalability of the multilevel versions of algorithm $b i S B M$, in 
terms of the average total time spent by each algorithm. Considering all algorithms, the total experimental runtime was 112,198 seconds, or nearly 31 hours. Figure 87 shows how each algorithm contributed to the total time, in both absolute values (y-axis) and relative values (percentages shown on top of the bars). In the best case, $M L P b_{C S L}+b i S B M$ with $L=2$ executed in 2,808 seconds ( 0.78 hours or $2.5 \%$ of the total time), whereas the standard biSBM executed in 57,062 seconds (nearly 15.8 hours, or $50.9 \%$ of the total time), which is 20 times slower than its multilevel implementation.

Additionally, We assessed the impact of each multilevel phase, i.e., coarsening, solution finding executing biSBM on the coarsest network, and uncoarsening, on execution time. Figure 88 shows the relative contributions of each phase to the total time for processing all networks. The coarsening phase consumed nearly $0,2 \%$ of the average total time, whereas the execution of biSBM consumed the remaining 98,8\%. The time spent in the uncoarsening phase was thus negligible. These results provide empirical evidence on the negligible impact of the coarsening and uncoarsening phases on the scalability of the multilevel method, thus reinforced as a powerful strategy to scale network optimization algorithms.

Finally, to compare their execution times regardless of a particular application, we executed the coarsening algorithms on 1,000 synthetic weighted bipartite networks with community structure, generated with the following parameter configurations (VALEJO et al., 2019): $|V|=\left|V^{1}\right|+\left|V^{2}\right|$ varying in the range $[500,10,000]$ at increments of 500; noise level $n$ varying in the range $[0.1,0.4]$ at increments of 0.05 ; number of communities $c=0.01|V|$, and network density $d=0.3$.

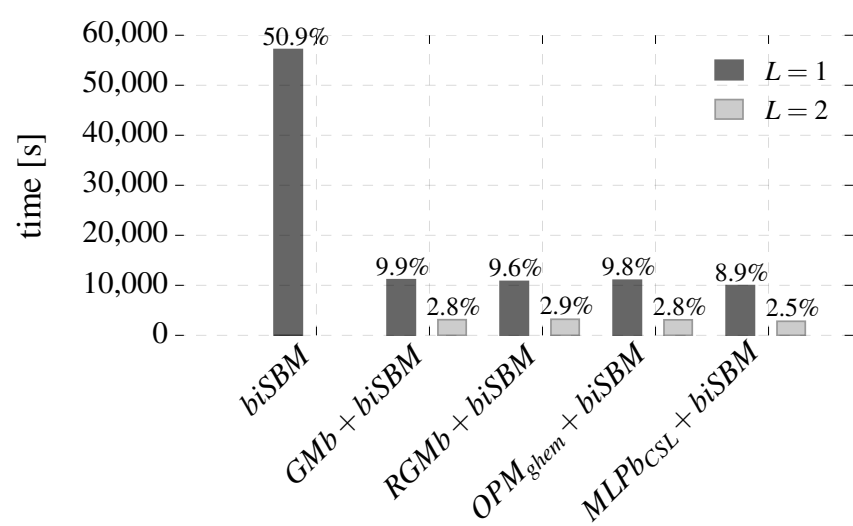

Figure 87 - Absolute and relative contribution of each algorithm to the total time of the experiments. The total runtime (average of 10 executions) is equal to 112,198 seconds, or 31 hours.

Source: Elaborated by the author.

Figure 89 shows the absolute runtime (in seconds) of each coarsening algorithm in each network. Values refer to average times relative to 10 executions; each algorithm iterated up to 10 coarsening levels. One notices a linear correlation between runtime and number of vertices $|V| . R G M b$ yielded the shortest time and $M L P b_{C S L}$ the longest, whereas algorithms $O P M_{h e m}$ and $G M b$ show very similar behavior regarding execution times. 

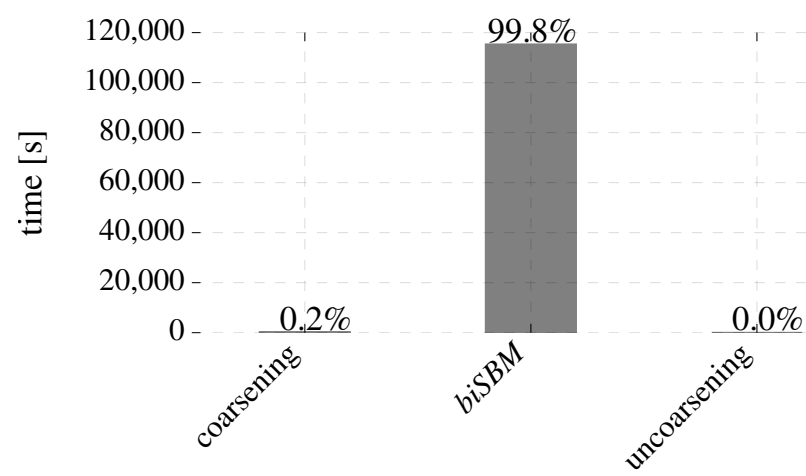

Figure 88 - Average absolute (y-axis) and relative contribution (percentage of execution time) of each multilevel phase in all networks.

Source: Elaborated by the author.

Besides network size, algorithm $M L P b_{C S L}$ depends on the number of iterations required prior to convergence. In small networks, few labels updates occur at each iteration, whereas in larger networks the probability of label updates is higher. Therefore, increasing $|V|$ directly impacts algorithm convergence and its asymptotic complexity. Indeed, according to Figure 89, differences are small in execution times of $M L P b_{C S L}$ relative to the other algorithms in networks with up to 40,000 vertices, and increases gradually as $|V|$ increases.

One also observes in Figure 89 that $M L P b_{C S L}$ has similar asymptotic complexity but higher execution time compared to the other algorithms. Interestingly, in the first experiment the lowest execution time was obtained with $M L P b_{C S L}+b i S B M$. Indeed, $M L P b_{C S L}$ builds more robust networks at the cost of a small loss in the topological structure. Therefore, biSBM requires less iterations to identify the best number of communities, which directly affects its execution time.

The previous analysis indicates that $M L P b_{C S L}$ yielded superior results compared to the state-of-the-art algorithms $O P M_{h e m}, R G M b$ and $G M b$ that operate on vertex pairs. It is also evident that, albeit they are fast, these algorithms are limited in their capability of preserving and revealing the underlying community structures in the networks. In the following study we investigate the behavior of $M L P b_{C S L}$ in the community detection problem. Our goal is to verify the quality of the super-vertices computed and the algorithm's adaptability to handle complex tasks.

\subsubsection{Community detection}

We assessed the behavior of $M L P b_{C S V}$ in the community detection problem, comparing it with three well-established algorithms known to be fast and effective ${ }^{5}$, namely $L P$ (RAGHAVAN; ALBERT; KUMARA, 2007; BARBER; CLARK, 2009), Louvain (BLONDEL et al., 2008) and Fastgreedy (CLAUSET; NEWMAN; MOORE, 2004). The motivation for this empirical

5 Parallel or distributed algorithms have not been considered. 


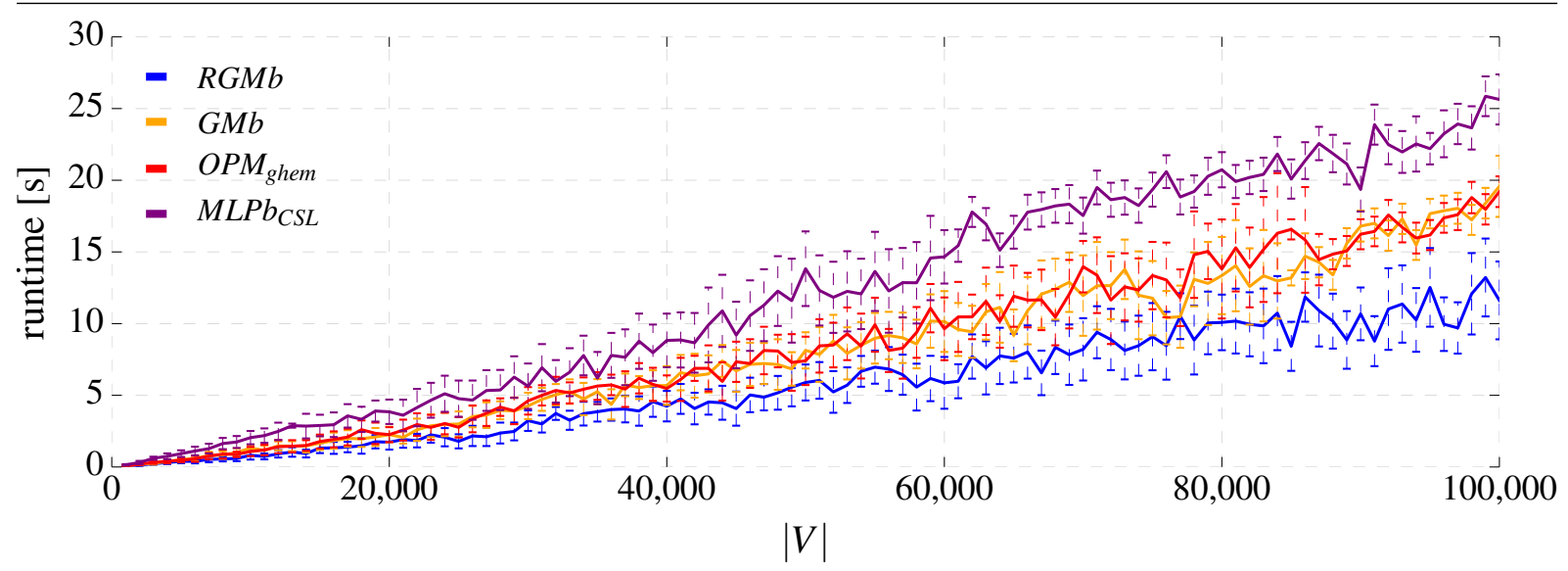

Figure 89 - Absolute runtime (seconds) of each coarsening algorithm to build a small representation of each of the 1,000 original synthetic networks.

Source: Elaborated by the author.

study is to assess the quality of the coarsening obtained with the algorithm, verifying whether the super-vertices it creates are comparable to the communities identified by reputed network partitioning algorithms from the literature. Since highly quality communities are expected to group vertices with similar properties, function or meaning, expressed as neighborhood or topological patterns, abstracting super-vertices as communities provides a reliable mechanism to verify their representativeness.

Louvain is a multilevel algorithm that relies on Newman's modularity maximization to infer the best community structure; $L P$ is a label propagation algorithm that maximizes modularity; and Fastgreedy is a hierarchical agglomeration algorithm based on modularity maximization. The number of communities is not specified a priori in these algorithms, therefore, they have been evaluated considering the number of communities they inferred. The selected algorithms are fast, with asymptotic complexity close to the $M L P b$, memory-efficient and known to yield meaningful community structures in practice. Moreover, reliable and optimized Python implementations are available in the Networkx library ${ }^{6}$.

Louvain, Fastgreedy and LP have been applied to the one-mode projections (see Fortunato (2010)) of the tested bipartite networks following the guidelines given in Chessa et al. (2014), Alzahrani and Horadam (2016) and Cann, Weaver and Williams (2019). Furthermore, considering that the three algorithms rely on modularity maximization and $M L P b_{C S V}$ relies on assessing neighborhood similarity, in order to ensure a fair comparison we adopted the well-known quality metric Conductance $(C)$, rather than modularity. Conductance assesses the separability of a community from the rest of the network (CHAKRABORTY et al., 2017), measured by the ratio between the number of its internal versus external edges. $C$ values are normalized in the range $[0,1]$, where 1 is the optimal score. We also measured runtimes of the algorithms.

Employing $M L P b_{C S V}$ for community detection is straightforward. It suffices to coarsen

$6<$ https://networkx.github.io/> 
the network to a defined number of super-vertices (using the CSV strategy, Alg. 23), and assign to each super-vertex a unique label. Super-vertex labels are then assigned to their corresponding predecessor vertices at each coarsening level along the projection phase, until the original network. Finally, vertices assigned the same label define the resulting communities (revisit Figure 82).

In assessing the algorithm we considered six real bipartite networks representative of diverse topological properties, available at $\mathrm{KONECT}^{7}$ (the Koblenz Network Collection). We took the largest connected component of each network, for which the size of each layer $\left|V^{1}\right|$ and $\left|V^{2}\right|$, the number of edges $|E|$, the average degree $\kappa(G)$, and average clustering coefficient $\varsigma(G)$ are detailed in Table 18. The upper bound parameter was set as $\mu=0.3$ to allow for slightly unbalanced super-vertices.

The number of desired communities $k$ determines the number of super-vertices in the coarsest network, i.e. $\zeta=k$. We used the following settings: $k=5$ for Unicodelang and Moreno, $k=10$ for IeeeVis, $k=20$ for N-reactome, $k=35$ for Condmat, and $k=40$ for Dbpedia, i.e., we adopt higher values for $k$ as network sizes increase.

Table 18 - Properties of the largest connected component of the real-world bipartite networks employed in the experimental evaluation.

\begin{tabular}{lrrrrr} 
Dataset & $\left|V^{1}\right|$ & $\left|V^{2}\right|$ & $|E|$ & $\kappa(G)$ & $\varsigma(G)$ \\
\hline Unicodelang & 248 & 610 & 1,249 & 2.91 & 0.43 \\
Moreno & 754 & 509 & 1,377 & 2.18 & 0.42 \\
IeeeVis & 3,919 & 2,378 & 8,868 & 2.82 & 0.35 \\
N-reactome & 8,788 & 15,433 & 41,087 & 3.39 & 0.32 \\
Condmat & 13,861 & 19,466 & 53,628 & 3.21 & 0.34 \\
Dbpedia & 54,909 & 19,866 & 98,895 & 2.64 & 0.45 \\
& \multicolumn{5}{l}{ Source: Research data. }
\end{tabular}

Table 19 summarizes the separability scores $C$ relative to each algorithm (average values over 10 executions). Both $M L P b_{C S V}$ and Louvain yielded the best $C$ values on 3 out of the 6 networks. $L P$ performed best on a single network, while Fastgreedy did not perform best on any of them.

A Friedman test and a Nemenyi post-hoc test at the 95\% confidence level (DEMSAR, 2006) have been applied to the results in Table 19 to verify statistical significance in the observed differences in algorithm performance; results are shown in Figure 90.

The final rows in Table 19 inform the average rank of the Friedman statistical test and the corresponding rank of the algorithm. According to the Nemenyi statistics, the critical value for comparing the mean-ranking of two different algorithms at 95 percentile is 1.91, i.e. significant

$7<$ http://konect.uni-koblenz.de/> 
Chapter 6. A coarsening method for labeled and unlabelled bipartite network via weight-constrained 186

label propagation

Table $19-C$ scores, average rank and rank of the four algorithms in 6 real-world networks. The values correspond to average values over 10 executions.

\begin{tabular}{lrrrr}
\multirow{2}{*}{ Dataset } & \multicolumn{5}{c}{ Algorithm } \\
\cline { 2 - 5 } & $M L P b_{C S V}$ & Louvain & LP & Fastgreedy \\
\hline Unicodelang & $0.471(3 \mathrm{rd})$ & $0.439(4 \mathrm{th})$ & $0.571(1 \mathrm{st})$ & $0.524(2 \mathrm{nd})$ \\
Moreno & $0.934(1 \mathrm{st})$ & $0.894(2 \mathrm{nd})$ & $0.618(4 \mathrm{th})$ & $0.874(3 \mathrm{rd})$ \\
IeeeVis & $0.829(1 \mathrm{st})$ & $0.829(1 \mathrm{st})$ & $0.522(4 \mathrm{th})$ & $0.811(3 \mathrm{rd})$ \\
Nreactome & $0.561(1 \mathrm{st})$ & $0.547(2 \mathrm{nd})$ & $0.372(4 \mathrm{th})$ & $0.408(3 \mathrm{rd})$ \\
Condmat & $0.812(2 \mathrm{nd})$ & $0.819(1 \mathrm{st})$ & $0.527(4 \mathrm{th})$ & $0.802(3 \mathrm{rd})$ \\
Dbpedia & $0.831(2 \mathrm{nd})$ & $0.882(1 \mathrm{st})$ & $0.518(4 \mathrm{th})$ & $0.829(3 \mathrm{rd})$ \\
\hline Average & 0.74 & 0.521 & 0.735 & 0.708 \\
Average Rank & 1.75 & 1.917 & 3.500 & 2.833 \\
Rank & $1 \mathrm{st}$ & $1 \mathrm{st}$ & $3 \mathrm{rd}$ & $2 \mathrm{nd}$ \\
& Source: Research data. & &
\end{tabular}

differences are above this value. The differences observed are not statistically significant, as indicated by the bold line in Figure 90 . Nevertheless, $M L P b_{C S V}$ was ranked first followed by Louvain, Fastgreedy and $L P$ in last. We conclude that $M L P b_{C S V}$ achieved competitive accuracy with Louvain, Fastgreedy and LP.

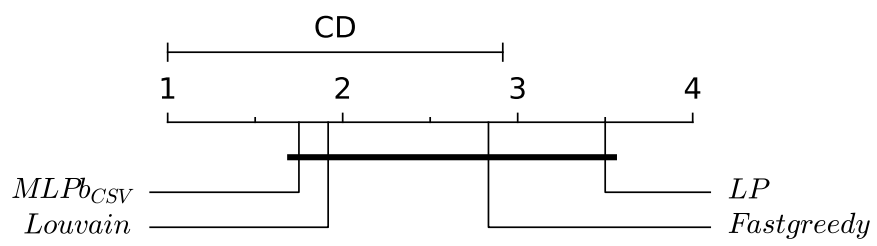

Figure 90 - Critical difference diagram of the results in Table 19.

We also computed algorithm execution times on each individual network, shown in Table 20 , where values refer to the average values over 10 executions in each network. $M L P b_{C S V}$ runtimes were the lowest in all networks, with speedups of aroud 2,5 relative to $L P, 3$ relative to Louvain, and 72,5 relative to Fast greedy.

$M L P b_{C S V}$ is fast because it operates directly in the bipartite network and separately on each layer, splitting the search space. Operating on one-mode projections, in contrast, implies in increasing considerably the number of edges handled by Louvain, LP and Fast greedy, even if the number of vertices has been reduced by half. Moreover, optimizing Newman's modularity is computationally more expensive than just counting common 2-hop neighbors between vertices. $L P$ often requiring many iterations before convergence as it also optimizes modularity locally on all vertices.

The results obtained allow us to conclude that $M L P b_{C S V}$ is faster than the algorithms Louvain, Fastgreedy and $L P$ and its super-vertices are representative of the community structures 
Table 20 - Execution times (seconds) of each algorithm on each network. We disregarded the time spent on read, write and one-mode projection operations.

\begin{tabular}{lrrrr}
\multirow{2}{*}{ Dataset } & \multicolumn{4}{c}{ Algorithm } \\
\cline { 2 - 5 } & $M L P b_{C S V}$ & Louvain & LP & Fastgreedy \\
\hline Unicodelang & 0.196 & 0.3721 & 0.1861 & 1.3123 \\
Moreno & 0.0544 & 0.1263 & 0.1161 & 0.2994 \\
IeeeVis & 0.5091 & 1.2587 & 0.9506 & 10.1857 \\
N-reactome & 12.386 & 41.3638 & 34.5531 & 148.8097 \\
Condmat & 4.3236 & 10.1867 & 10.1815 & 418.1561 \\
Dbpedia & 13.4365 & 54.9436 & 38.4371 & 1976.5222 \\
\hline Total & 35.345 & 102.662 & 87.552 & $2,559.533$ \\
& \multicolumn{5}{c}{ Source: Research data. }
\end{tabular}

found by these algorithms. Furthermore, although the algorithm has not been designed for community detection its application to this task is straighforward.

\subsubsection{Network coarsening for visualization}

Network visualization deals with the problem of creating visual representations of networked data that support user-driven exploratory investigation. Visualizations should have reduced visual complexity and be aesthetically pleasing, and are expected to assist users in gaining insight on the global and local behavior of a dataset and improve their comprehension of the data.

The popular node-link layouts face severe limitations (GHONIEM; FEKETE; CASTAGLIOLA, 2005). First, the drawings quickly become crowded as the number of vertices and edges easily exceed the number of display pixels available (GHONIEM; FEKETE; CASTAGLIOLA, 2005). Overlapping of the graphical elements depicting vertices and edges impair readability and user interaction. The high computational cost that prevents real-time rendering of large networks is an additional limitation of classic force-directed network drawing algorithms (DÍAZ; PETIT; SERNA, 2000; HACHUL; JüNGER, 2007; LANDESBERGER et al., 2011). These limitations are aggravated on bipartite networks, in which issues due to sparsity and complexity of topological structures can be even more severe than in unipartite networks.

Multilevel strategies have been successfully employed to compute node-link layouts of unipartite networks (ABELLO; HAM; KRISHNAN, 2006; Archambault; Munzner; Auber, 2008; Wong et al., 2009; BATAGELJ et al., 2011; DIAS et al., 2017; PERROT; AUBER, 2018). Other studies combine aggregation and clustering, which is an approach that resembles coarsening in that they also rely on creating simplified representations of the full network (DIAS et al., 2017; PERROT; AUBER, 2018). However, we are not aware of previous efforts of employing the multilevel method for visualizing bipartite networks. 
As network coarsening should preserve the network structures relevant for problem solving, we hypothesize it can provide a useful strategy to depict simplified views of large networks for visualization purposes. In this section we report an empirical investigation of this assumption. In particular, we illustrate how coarsening with $M L P b_{C S V}$ creates summarized node-link representations that preserve the major characteristics of an original network whilst reducing the amount of information displayed. We show several illustrative examples using synthetic and real-world networks with diverse topological properties. All the network drawings have been computed with the force-directed algorithm introduced by Eades (1984) and later improved by Kamada and Kawai (1989) and Fruchterman and Reingold (1991). We used the Igraph Library ${ }^{8}$ implementation and rendered drawings with a resolution of $800 x 800$ pixels.

Figure 91a depicts a synthetic sparse bipartite network with five balanced communities formed by 2,000 vertices $\left(\left|V^{1}\right|=\left|V^{2}\right|=1,000\right)$ and with extensive noise, i.e., many edges between communities. Due to its size, sparsity and noise, community boundaries are not clearly visible. Communities can be better distinguished using colors, as in Figure $91 \mathrm{~b}$, or the colors can be combined with a strategy of displacing the communities apart, as in Figure 91c. These are common strategies for rendering network drawings in several network analysis software, such as NetworkX ${ }^{9}, \operatorname{Igraph}^{8}, \mathrm{Gephi}^{10}$ or Cytoscape ${ }^{11}$. Nonetheless, the problems remain that computing the layout is extremely slow and the resulting layout shows severe overlapping of graphical elements. Such drawings are of limited use for purposes of interactive visualization.

$M L P b$ was executed to obtain a coarsened network with $|V|=200\left(\left|V^{1}\right|=\left|V^{2}\right|=100\right)$, drawn in Figure 91d. The five communities and their boundaries are very explicit in the reduced network. Outlier and isolated vertices are easily identifiable and constrained within the display limits. Furthermore, cross-edges between communities and the two types of vertices can be distinguished. The coarser representation reduces the clutter and the amount of information displayed, whilst still conveying the essential global structure of the full network.

This behavior can be explained by the data structure created by the contraction algorithm. The edge weights in a neighborhood (intra-community edges) increase at each coarsening level, unlike the weights of edges crossing the neighborhoods (inter-community edges). Furthermore, the weight of a super-vertex depends on the number of predecessors, thus lower-weights can indicate a small or less significant neighborhood. Therefore, the data structure emphasizes the community patterns in the network layout, since it is used by the force-directed algorithms in computing the layout.

As another example, Figure 92a shows a synthetic network with regular degree distribution and no community structure, with $|V|=5,000$.

\footnotetext{
$8<$ https://igraph.org/>

$9<$ https://networkx.github.io/>

$10<$ https://gephi.org/>

$11<$ https://cytoscape.org/>
} 


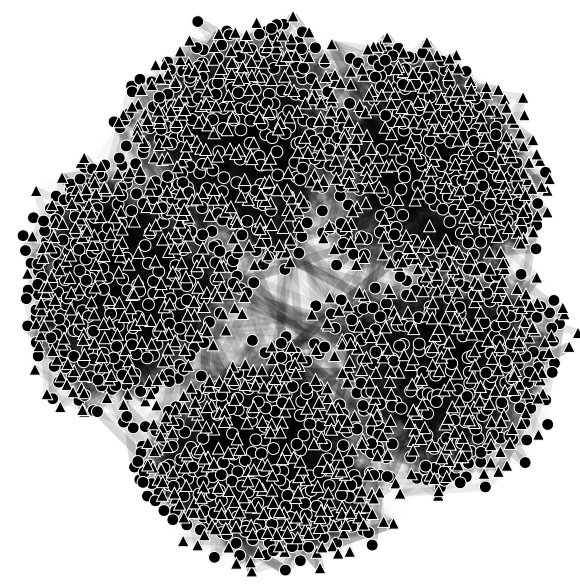

(a)

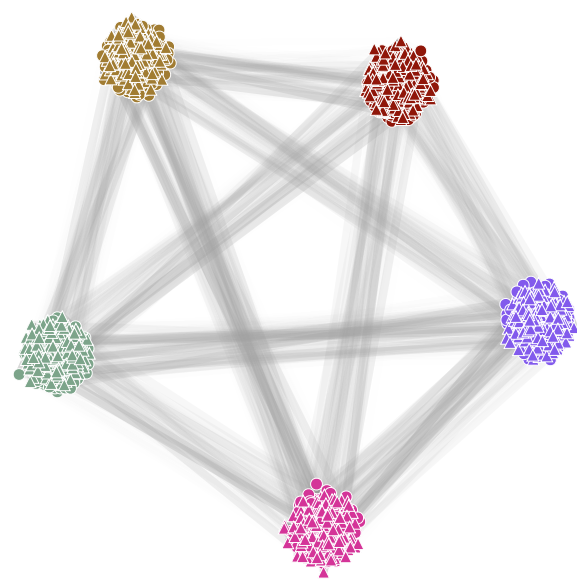

(c)

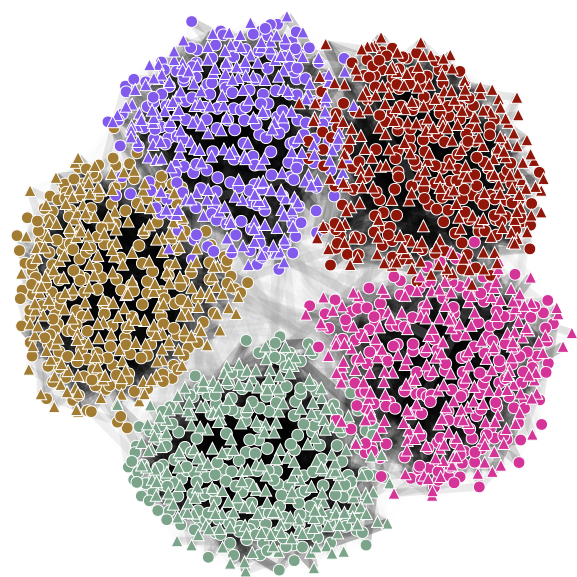

(b)

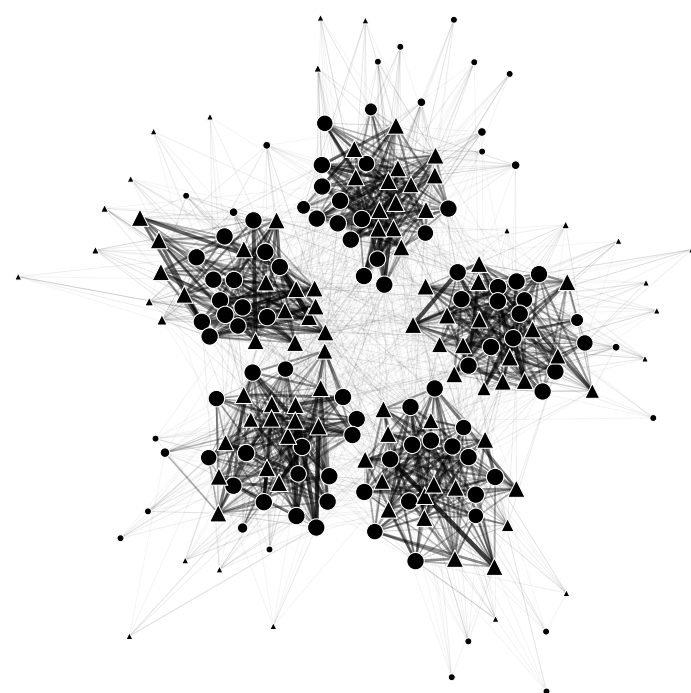

(d)

Figure 91 - Multiple layouts of a synthetic sparse bipartite network with $|V|=2,000$ vertices $\left(\left|V^{1}\right|=\right.$ $\left.\left|V^{2}\right|=1,000\right)$ distributed into 5 balanced communities with extensive noise. All layouts have been computed with the force-directed algorithm; in (b) and (c) the communities are identified with colors. (d) depicts the layout of the network coarsened to $|V|=200$ vertices with $M L P b_{C S V}$.

Source: Elaborated by the author.

Figure 92b depicts a coarsened version obtained executing $M L P b_{C S V}$ with $\left|V^{1}\right|=\left|V^{2}\right|=$ 400. Drawing the original network takes around 1,000 seconds, against 10 seconds to draw the coarser network. This example illustrates that $M L P b_{C S V}$ does not introduce topological structures that are not present in the original network. Rather, the drawing of the reduced network is actually more effective in revealing outliers such as the highest and lowest degree vertices. For purposes of an making an initial assessment of its topological structure, it is more convenient to observe a coarsened version of the network than waiting a long time for a drawing of the full model that reveals limited information and does not support interaction.

Indeed, smooth user navigation is a critical challenge in visualization of large networks, 


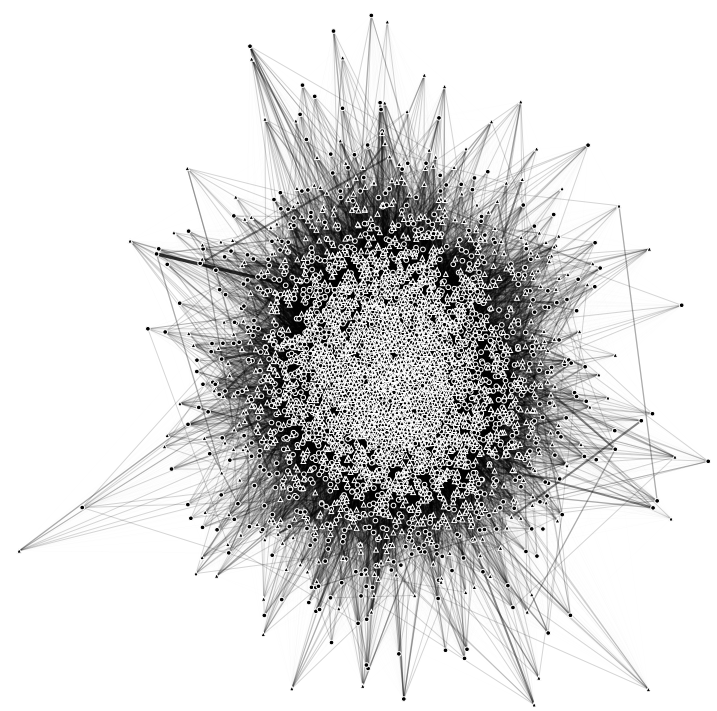

(a)

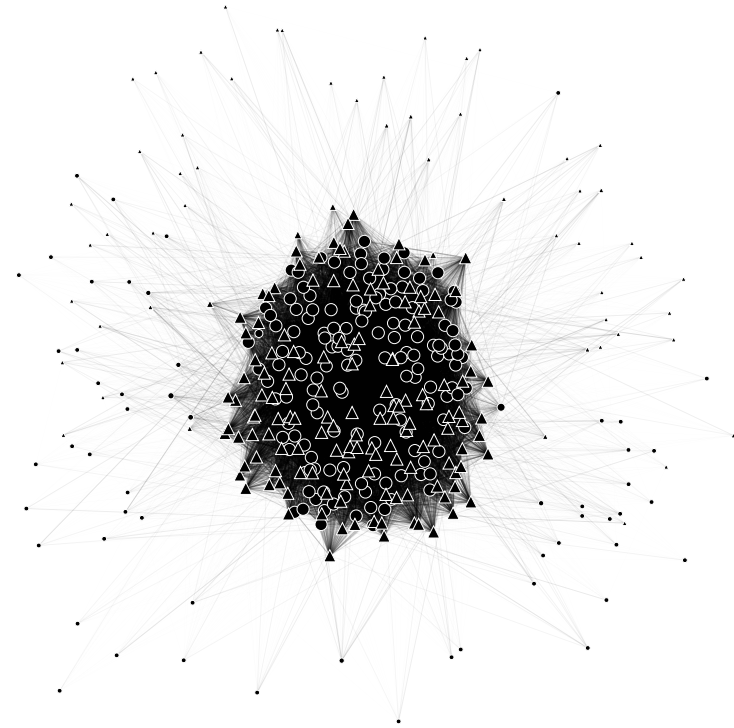

(b)

Figure 92 - Synthetic bipartite network and distinct drawings obtained with a force-directed algorithm: (a) shows a regular network with no community structure, regular degree distribution and $|V|=5,000$; (b) illustrates a coarser representation, obtained with $M L P b_{C S V}$, with $\left|V^{1}\right|=$ $\left|V^{2}\right|=200$.

as users expect to grasp information relative to both local and global contexts (GHONIEM; FEKETE; CASTAGLIOLA, 2005). In these scenarios, $M L P b_{C S V}$ could support interactive visualization of large-scale bipartite networks by means of navigation over the hierarchy of coarsened networks, which would be kept in an appropriate data structure.

A super-vertex could be uncoarsened into its predecessor-vertices on demand, i.e., its content is "exploded" in response to a user interaction. Another possible strategy would be to redraw a selected neighborhood at a user-defined coarsening level, with the coarsening computed on the fly.

We further illustrate the behavior of $M L P b_{C S V}$ coarsening in two well-known real-world bipartite networks. In the corresponding network drawings, vertex weight is mapped to size and color of the graphical markers depicting super-vertices. We adopted a black-to-red color scale, so that bright red markers denote high-weight super-vertices.

Moreno Crime is a social network with scale-free behavior relating 754 crime suspects or victims with 509 crimes (Figure 93); N-reactome is a biological network with modular topology with 8,788 proteins and 15,433 interactions in the species Homo Sapiens (Figure 94). These networks are available in the Koblenz Network Collection (KONECT) ${ }^{12}$.

Figure 93a shows the original Moreno Crime network and Figures 93b, 93c and 93d illustrate it at three gradually increasing levels of coarsening, respectively with $|V|=800\left(\left|V^{1}\right|=\right.$ 500 and $\left.\left|V^{2}\right|=300\right),|V|=500\left(\left|V^{1}\right|=300\right.$ and $\left.\left|V^{2}\right|=100\right)$ and $\left|V^{1}\right|=150\left(\left|V^{1}\right|=100\right.$ and 


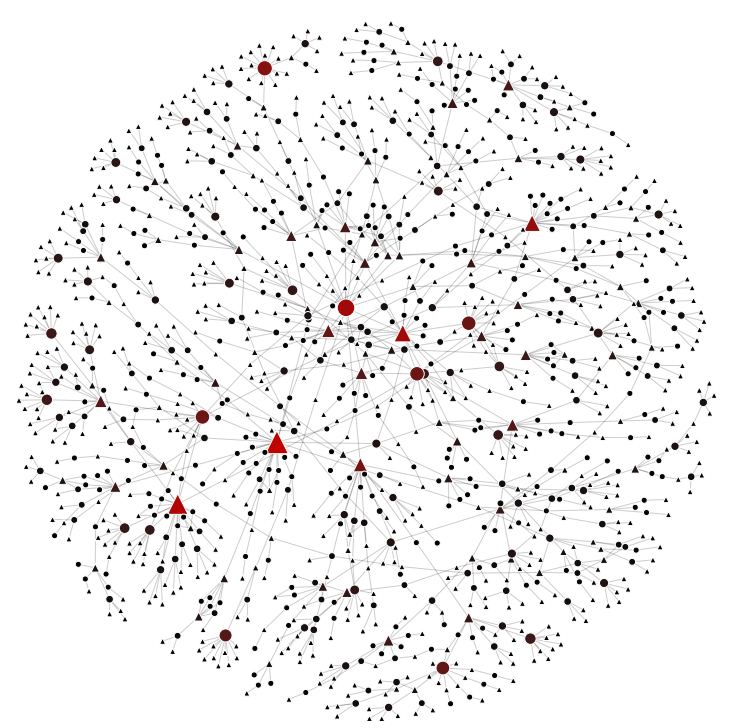

(a)

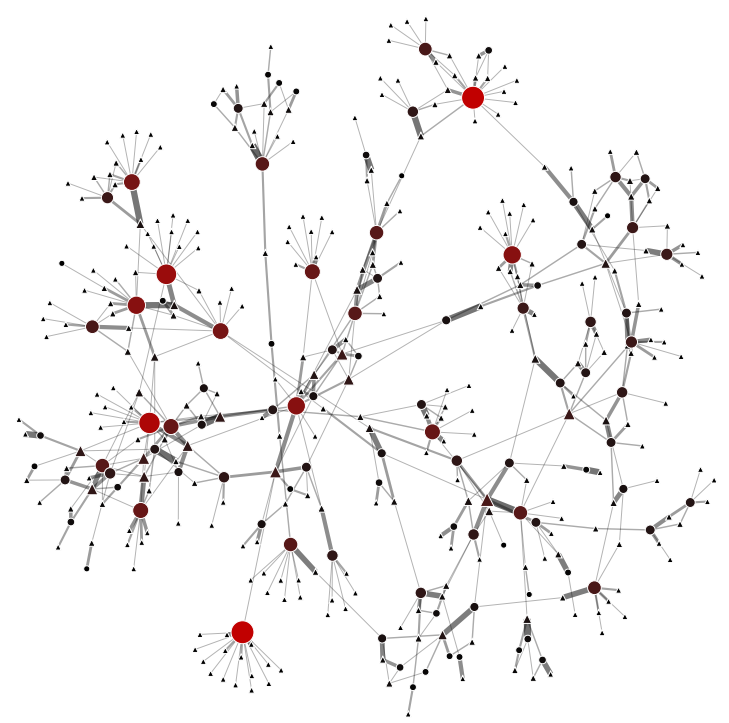

(c)

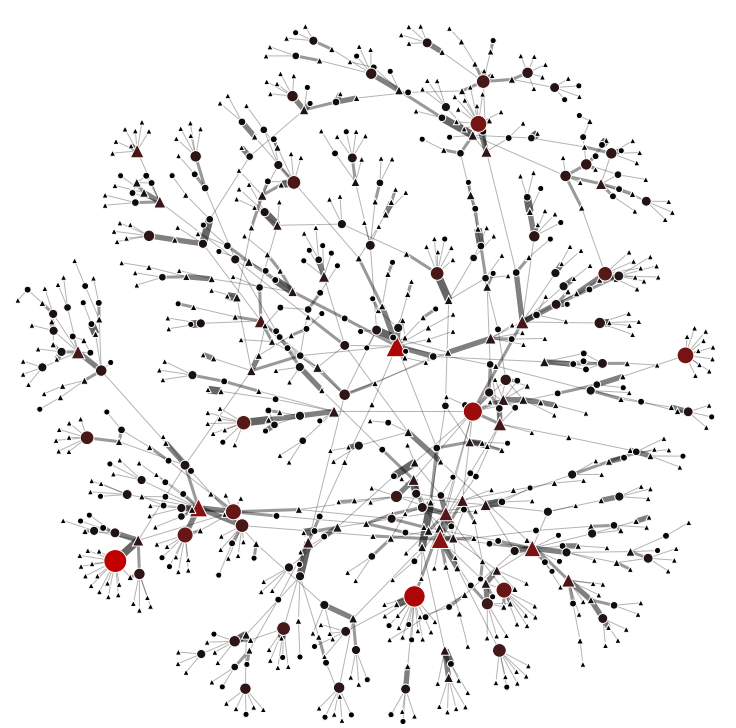

(b)

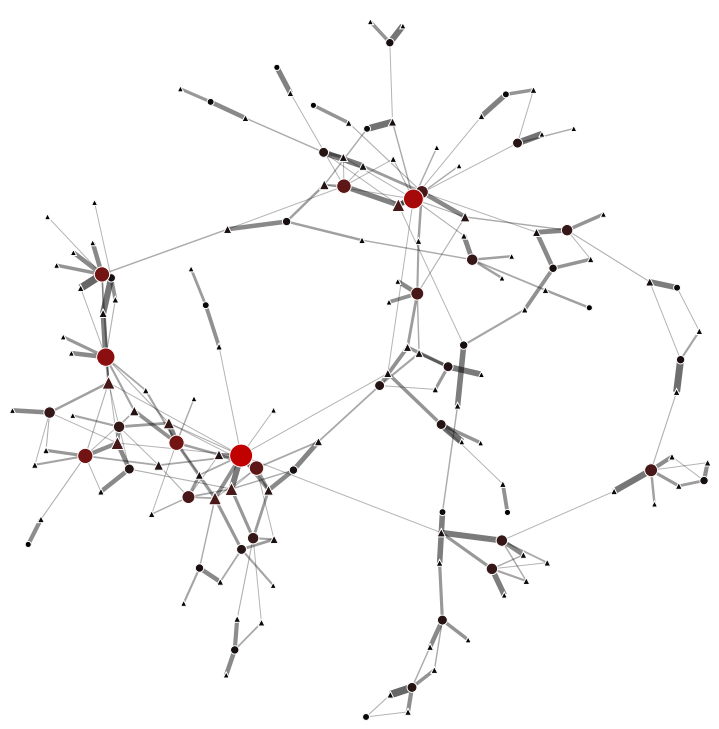

(d)

Figure 93 - Moreno Crime social network with scale-free behavior, which relates 754 criminal case suspects or victims with 509 crimes. (a) shows the original network, (b), (c) and (d) illustrate it at three increasing levels of coarsening, with $|V|=800,|V|=400$ and $|V|=150$, respectively.

Source: Elaborated by the author.

$\left|V^{2}\right|=50$ ). This network's degree distribution follows the power-law relationship characteristic of scale-free networks. This behavior is reasonably preserved in the drawings of the coarsened models, up to a certain level, e.g., the presence of both hubs and low-degree vertices is evident in Figures $93 b$ and $93 c$.

Figure 94a shows the N-reatome network and Figures 94b to 94i illustrate it at eight increasing coarsening levels, with the number of vertices set respectively to $|V|=18,000$ $\left(\left|V^{1}\right|=6,000\right.$ and $\left.\left|V^{2}\right|=12,000\right),|V|=10,000\left(\left|V^{1}\right|=\left|V^{2}\right|=5,000\right),|V|=5,000\left(\left|V^{1}\right|=\right.$ $\left.\left|V^{2}\right|=2,500\right),|V|=2,000\left(\left|V^{1}\right|=\left|V^{2}\right|=1,000\right),|V|=1,600\left(\left|V^{1}\right|=\left|V^{2}\right|=800\right),|V|=1,000$ 


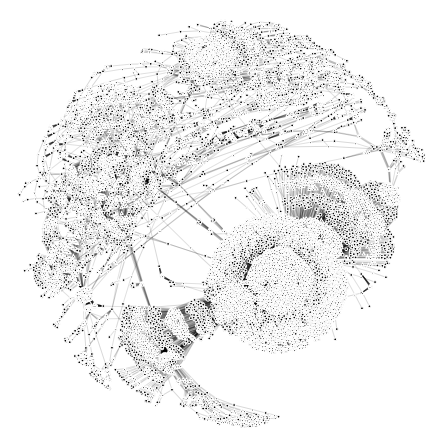

(a)

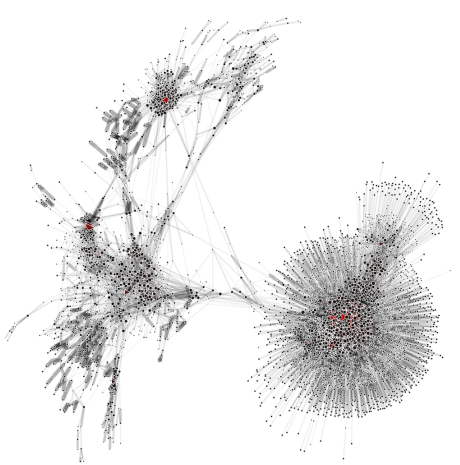

(d)

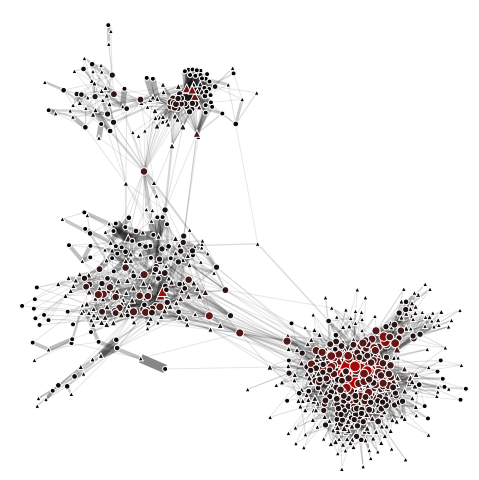

(g)

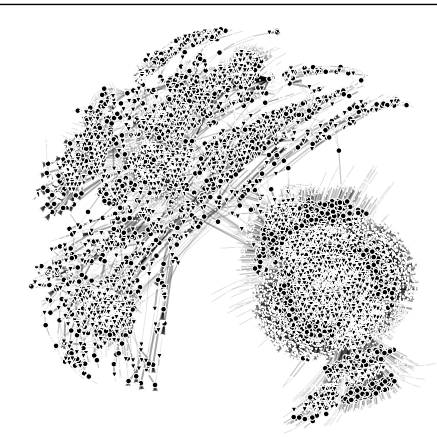

(b)

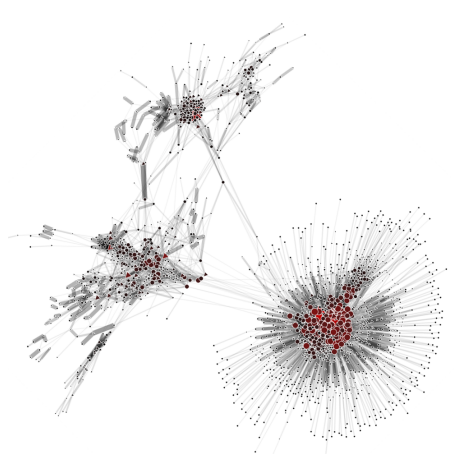

(e)

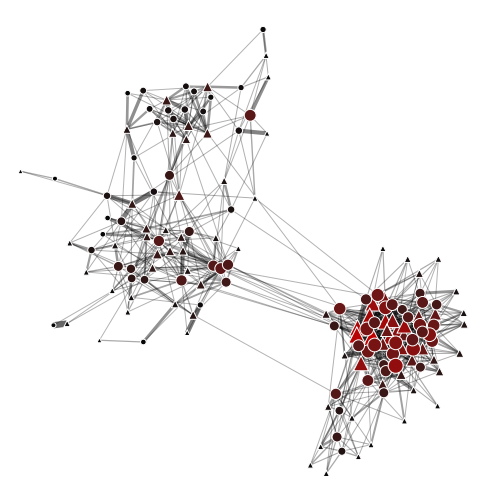

(h)

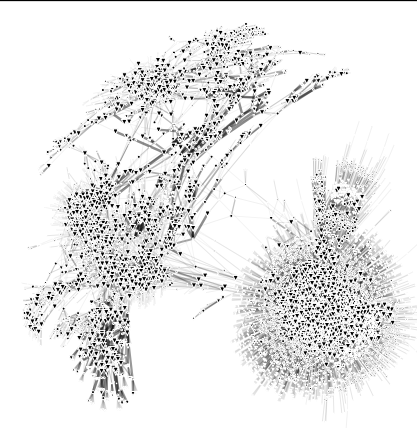

(c)

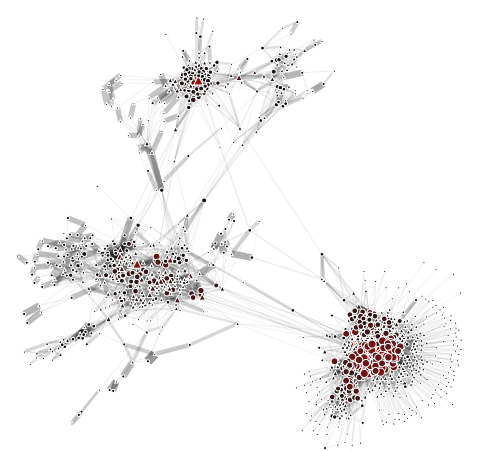

(f)

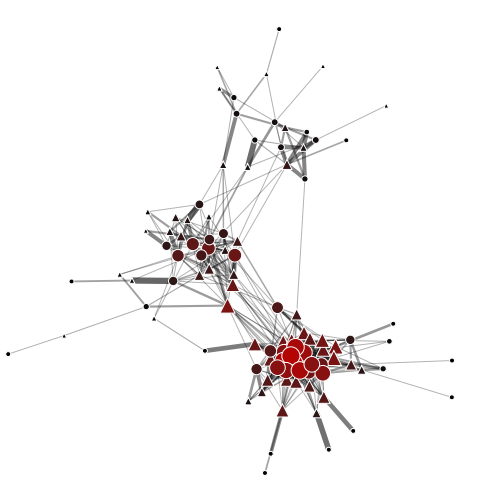

(i)

Figure 94 - N-reatome biological network with modular topology, depicting 8,788 proteins and 15,433 interactions in the species Homo Sapiens. (a) shows the original network and (b), (c), (d), (e), (f), (g), (h) and (i) illustrates the network at eight increasing levels of coarsening, with $|V|=12,000,|V|=10,000,|V|=5,000,|V|=2,000,|V|=1,600,|V|=1,000,|V|=200$ and $|V|=100$, respectively.

Source: Elaborated by the author.

$\left(\left|V^{1}\right|=\left|V^{2}\right|=500\right),|V|=200\left(\left|V^{1}\right|=\left|V^{2}\right|=100\right)$ and $|V|=100\left(\left|V^{1}\right|=\left|V^{2}\right|=50\right)$. This network has a modular topology with four noticeable modules. Again, the original topological structure and overall organization are preserved up to a certain level. At more advanced coarsening levels the original topological properties are lost as more vertices are collapsed into super-vertices, as observed mainly from $94 \mathrm{f}$ onwards. Nonetheless, three well-defined modules are still visible even in Figures 94g and 94h, and the essential global topology is still noticeable in Figure 94i. 
For purposes of comparison, in Figure 95 we show drawings of the N-reactome network coarsened with the $R G M b$ pairwise matching algorithm. Setting parameters $\rho$ (reduction factor) and $L$ (number of coarsening levels) is not intuitive and we executed the algorithm several times with different tentative settings before we succeeded in obtaining a coarsened network with around 100 vertices. With $\rho=0.5,11$ coarsening levels were necessary to yield $\left|V^{1}\right|=68$ and $\left|V^{2}\right|=56$ (Figure 95a). Adopting $\rho=0.1,50$ coarsening levels were necessary to yield $\left|V^{1}\right|=52$ and $\left|V^{1}\right|=53$ (Figure 95b). Lower values of $\rho$ imply in more coarsening levels to reach a target network size and additional memory to keep all the intermediate levels.

It is also evident that the choice of $\rho$ affects super-vertex balance, e.g., $\rho=0.5$ yielded more balanced super-vertices (Figure 95a) than $\rho=0.1$ (Figure 95b). A visual analysis reveals that the original global topological structures have been lost, an additional evidence that beyond favouring unbalanced super-vertices pairwise matching tends to degrade relevant topological structures. On the contrary, the models coarsened with $M L P b$, albeit losing detail, can actually reveal topological patterns that remain hidden, or blurred, in a drawing of the full network.

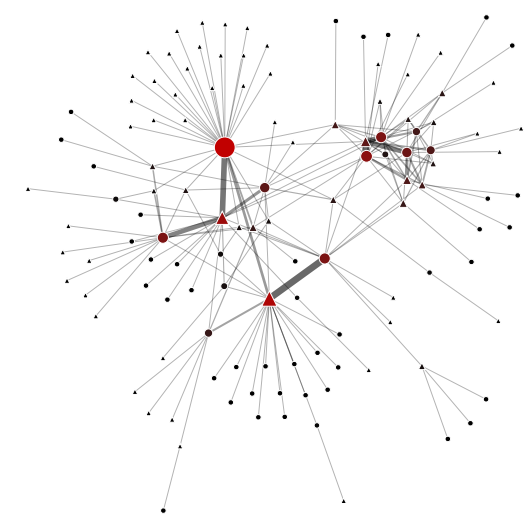

(a)

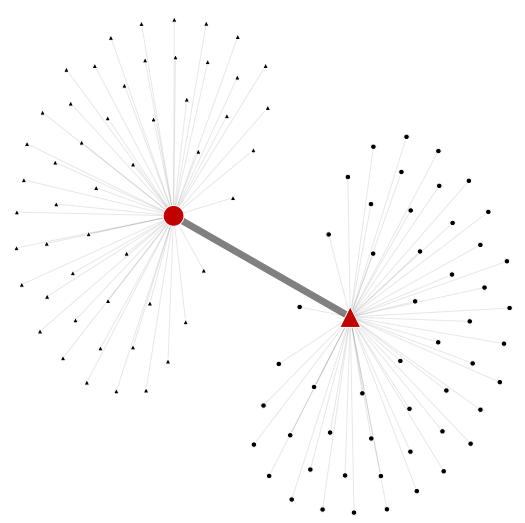

(b)

Figure 95 - Drawings of the N-reatome biological network after coarsening with the $R G M b$ matching algorithm. (a) illustrates the network obtained after 11 coarsening levels, with $\rho=0.5$, $\left|V^{1}\right|=68$ and $\left|V^{2}\right|=56$; (b) illustrates the network obtained after 30 coarsening levels, with $\rho=0.1$, with $\left|V^{1}\right|=52$ and $\left|V^{2}\right|=53$.

Source: Elaborated by the author.

We also investigated $M L P b$ coarsening of non-symmetric sparse matrices with hole topology. A network hole is defined as a loop formed by a vertex sub-set with only cyclic edges and no edges crossing the loop. Holes can indicate a special large-scale event occurring at a particular region, which may need monitoring. On communication networks, for instance, operation may be disrupted by sufficiently large holes (FUNKE, 2005). Petri et al. (2013) introduced a method to assist topological filtering methods by detecting particular structures, including network holes. Their method removes redundant edges to create reduced networks that fully preserve the topological information.

We show as example the network labeled as "HB/jagmesh7" from the SuitSparse Matrix 
Collection $^{13}$, shown in Figure 96a. Figures 96b and 96c illustrate the network at two levels of coarsening, obtained setting $\mu=0.1$ in algorithm $M L P b_{C S V}$ (since we want balanced supervertices). One notices the hole topology remains evident in the drawings of the coarser models. Our multilevel strategy can thus be useful, e.g., to verify the presence of holes inspecting a coarser version of a large-scale network, prior to performing complex or expensive tasks interactin with a view of the full model.

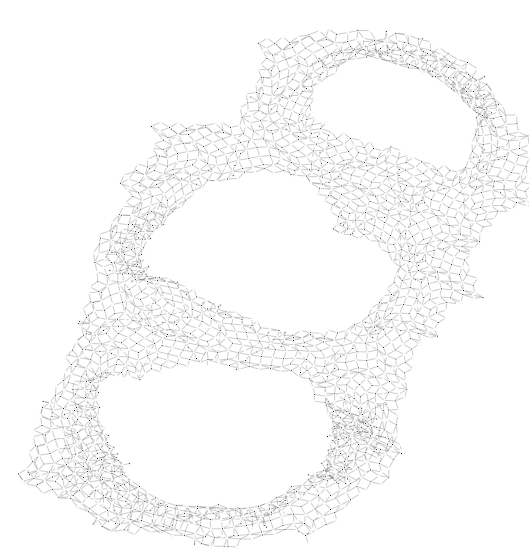

(a)

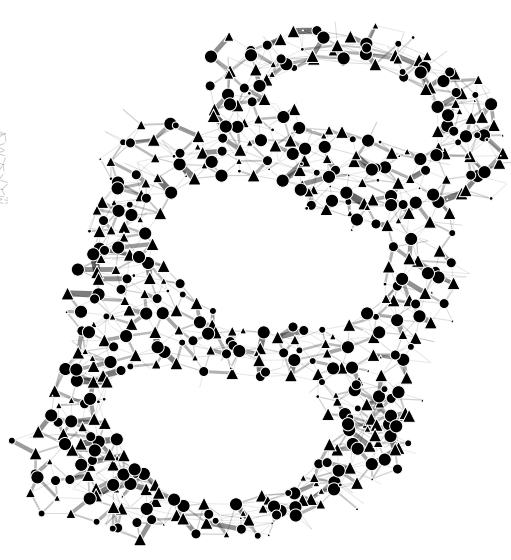

(b)

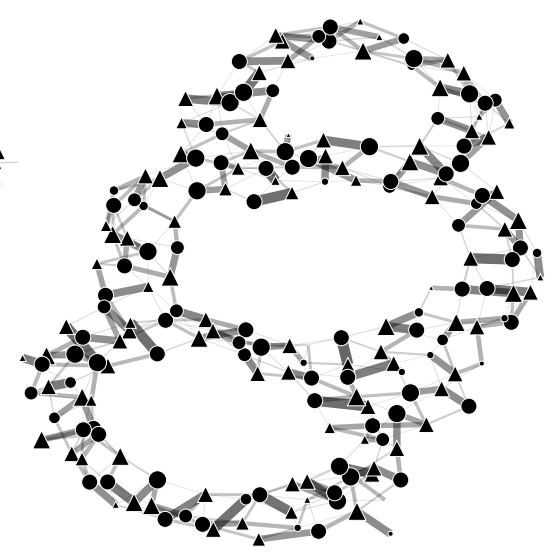

(c)

Figure 96 - Bipartite network with three holes: (a) shows the original network with $V^{1}=V^{2}=1,138$, (b) and (c) illustrate it at two increasing levels of coarsening, with $V^{1}=V^{2}=300$ and $V^{1}=V^{2}=100$, respectively.

Source: Elaborated by the author.

Interestingly, the coarsened networks show holes that are similar in size to those of the original network. Although holes are defined with fewer edges in a coarser network, super-edge weights derive from the weights of their predecessor edges, therefore the hole sizes are preserved in the force-directed layout. This example reinforces that $M L P b_{C S V}$ coarsening is robust to varied topological properties, as already exemplified in the scale-free, regular degree distribution and modular topological structures. Furthermore, its usage is more intuitive, as the user can specify a desired network reduction in terms of the number of vertices in each layer of the coarsest network.

According to Ghoniem, Fekete and Castagliola (2005), even in executing simple tasks such as locating a vertex or finding the edges between two vertices, human perceptual abilities are limited to small network sizes. As networks increase, users find it difficult to accomplish even simple data exploration tasks. Thus, early interaction with simplified representations of a network can prove more useful to support early assessments than drawing the full network and all its associated information.

\footnotetext{
13 available in $<$ https://sparse.tamu.edu/>
} 


\subsection{Concluding Remarks}

Inspired by the potential of general-purpose multilevel strategies to scale optimization problems in networks we introduced a new coarsening strategy via weight-constrained label propagation for bipartite networks. We propose two variations that differ in the input parameters required: $M L P b_{C S L}$ that implements a standard formulation of the multilevel strategy, in which the user directly sets the number of desired levels and the network reduction factor; and $M L P b_{C S V}$, which automatically creates the coarsening hierarchy given the desired number of vertices in the coarsest network.

We conducted three empirical studies for purposes of validating the $M L P b$ coarsening strategy. The first study describes its application to optimize the high-cost community detection algorithm $b i S B M$. The results show that $M L P b_{C S L}$ can drastically speedup this classic community detection algorithm, while preserving solution quality. The second study applied $M L P b_{C S V}$ directly as a community detection algorithm, comparing the results thus obtained with those from three popular community detection algorithms knwon to be fast. Again, the results indicate that the $M L P b_{C S V}$ coarsening algorithm can yield equivalent solutions in terms of quality, at a lower computational cost.

Finally, in a third study of a more exploratory nature $M L P b_{C S V}$ was employed in connection with force-directed algorithms to display simplified drawings of several large or very large networks. The primary goal of this study was to illustrate the coarsening behavior of $M L P b_{C S V}$ in networks with distinct characteristics and topological properties. As a side effect, we argue that coarsening can be a useful resource in the context of interactive data visualization to support exploratory tasks on datasets represented as bipartite networks. There is evidence that the networks coarsened with $M L P b_{C S V}$ preserve the global topological patterns of the original models and allow a force-based drawing algorithm to build readable summarized layouts of the original. This strategy could be embedded into a visualization system, in connection with appropriate interaction functions, to enhance the user experience in scenarios that require interacting with node-link representations of large networks.

Identifying the level of coarsening which yields a suitable trade-off between algorithmic accuracy and execution times is a critical issue in applying the multilevel method. Currently, this is determined with ad-hoc investigations conducted in each application problem and dataset, but it is certainly an issue that deserves further investigation. $M L P b$ is the first coarsening solution to admit setting an expected size for the coarsest network, which is more intuitive than informing a number of coarsening levels. It is still necessary, however, to find out whether the coarsening applied is compatible with obtaining a good quality solution.

Our algorithms support a general implementation of the multilevel method in bipartite networks, and as such can encourage novel approaches to solve other problems beyond those addressed in this paper. We are particularly interested in its usage on supervised and unsupervised 
classification tasks, as well as in interactive visualization of large networks. Also as future work we plan to extend the algorithms to handle problems defined in heterogeneous networks, where edges connect vertices of multiple types. The method's applicability could be thus extended to problems described by document-word networks indicating multiple types of associations, e.g. document-word, word-word, and document-document; or networks characterizing relationships between words, documents and authors. Investigating distributed or parallel paradigms for implementing the method is also a challenging research topic. An implementation of the proposed algorithms can be downloaded from $<$ https://github.com/alanvalejo/mlpb $>{ }^{14}$.

14 The software will be made available immediately after paper acceptance. 
CHAPTER

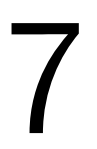

7

\section{CONCLUSION}

The multilevel method is a general problem-solving strategy with great applicability and potential to scale optimization problems modeled in networks. Motivated by its potential, this research investigated and designed a general multilevel framework capable of handling bipartite networks which is sufficiently flexible to be customized or adapted to distinct application domains. The major motivation for this research is that classical multilevel methods are not directly applicable to bipartite networks. To handle this gap were identified possible conflicts related to information conveyed by bipartite networks and standard coarsening approaches, outlining three hypotheses which guided this research:

Hypothesis 1: The first hypothesis considers that the effectiveness of a multilevel strategy designed to handle bipartite networks depends on two factors: The coarsening will not match vertices of different kinds and layers (either one of them or both) should be processed independently, since each layer represents different entities and may have distinct structural and functional features. Furthermore, this strategy allows adopting distinct coarsening and refinement algorithms, or distinct parameterizations, in processing each layer. Based on these observations, this research introduced a novel framework that incorporates the aforementioned assumptions, called $M O b$ (Multilevel Optimization in bipartite networks).

Hypothesis 2: The second hypothesis admits that the one-mode projection allows classic coarsening algorithms to be evaluated in bipartite networks, since it creates separate projections of each vertex type and therefore avoids collapsing of vertices of the same type and allows independent processing of the layers. Accordingly, the OPM (One-mode projectionbased multilevel approach) method was designed that allows applying standard matching algorithms (such as the well-known HEM, GHEM and RM) to bipartite networks.

Hypothesis 3: The third hypothesis acknowledges the use of the 2-hop vertex neighborhood information to support a cost-effective matching implementation on bipartite networks. 
Whereas vertices in the same layer can be accessed from each other through even-sized hop neighborhoods, the 2-hop neighborhood of a selected vertex comprises its closest unconnected neighbors of the same type. This concept allows designing matching algorithms which operate directly on a bipartite structure and suppresses the need of one-mode projections. Three matching algorithms were proposed, namely $G M b$ (greedy matching for bipartite networks), $R G M b$ (random greedy matching for bipartite networks) and $M L P b$ (matching via weight-constrained label propagation in bipartite networks). Although it has empirically proven that the matching process works with 2-hop, experiments with different hops were not performed since the scalability was a critical issue in this research.

To prove the usefulness and flexibility of the proposed methods and to provide compelling empirical evidence of the validity of the previous hypotheses, an empirical analysis was conducted in multiple scenarios obtained on a vast set of synthetic and real-world networks.

Optimization: We used the proposed method to optimize two high-cost bipartite community detection algorithms, namely BiSBM and $L P A w b+$. Results from an empirical investigation on an expressive set of synthetic and real-world networks show a considerable speed up with no significant loss in solution quality, as compared to the standard algorithms. Furthermore, to resume the convergences between hypotheses 1 and 2, OPM (one-mode projection-based) and $M O b$ (based directly on the bipartite structure) were empirically compared and, although there is no significant statistical difference between the strategies, $M O b$ performs better in a general context. From this analysis, $O P M$ reached good performance, i.e., it is admitted the use of established coarsening methods in bipartite networks. Nevertheless, it should be used with care, since some information may be lost. For instance, in an author-paper network, many papers are written by a single author, and this information is lost in the one-mode projection since it only captures the collaborations.

Comunity Detection: We also show the strategy can be easily adapted to identify community structures in bipartite networks, yielding solutions that are statistically equivalent, regarding the accuracy, to well-known community detection algorithms, namely, Louvain, $L P$ and FastGreedy.

Dimensionality reduction: To demonstrate the ability to process layers independently, $M d r$ (multilevel dimensionality reduction) was proposed. Employed to perform dimensionality reduction in text classification it yielded encouraging results in terms of both runtime and accuracy as compared with a standard dimensionality reduction technique, namely PCA.

Visualization: Finally, we also employed the method in connection with force-directed network drawing algorithms to obtain informative layouts of large bipartite networks, in addition to discussing its potential for interactive visualization applications. 
In addition to its major contribution, two additional studies were presented in this thesis. It provides a critical survey that compiles a unified reference to the literature, organizing the contributions under a theoretical and conceptual framework. The thesis also includes a critical assessment of many strategies and choices available to implement a multilevel solution. Hopefully, it can contribute to reducing the effort required from newcomers to grasp the current state-of-the-art in multilevel optimization, inspire new directions of investigation and pave the development of novel applications.

Furthermore, to support the early development stages of the proposed algorithms and, later, an accurate assessment and comparison of alternative solutions, a benchmarking tool was developed, called $B N O C$, to synthesize large-scale bipartite network models that mimic a wide range of features from real-world networks. BNOC can be also useful for benchmarking other kinds of algorithms and applications beyond the multilevel context, e.g. community detection, link prediction or classification algorithms.

\subsection{Scientific contributions}

The following papers have been published or submitted as a direct result from this research and colaborations:

1. Valejo, A. and Filho, G. P. R. and Oliveira, M. C. F. and Ferreira, V. and Lopes, A. A.. One-mode projection-based multilevel approach for community detection in bipartite networks, 2018, Proceedings of the International Symposium on Information Management and Big Data.

2. Valejo, A. and Ferreira, V. and Oliveira, M. C. F. and Lopes, A. A.. Community detection in bipartite network: a modified coarsening approach, 2018, Proceedings of the International Symposium on Information Management and Big Data.

3. Valejo, A. and Filho, G. P. R. and Oliveira, M. C. F. and Ferreira, V. and Lopes, A. A.. Multilevel approach for combinatorial optimization in bipartite network, 2018, KnowledgeBased Systems.

4. Valejo, A. and Ferreira, V. and Fabbri, R. and Oliveira, M. C. F. and Lopes, A. A.. A critical survey of the multilevel method in complex networks, ACM Computing Surveys (CSUR), submitted paper.

5. Valejo, A. and Góes, F. and Romanetto, L. and Oliveira, M. C. F. and Lopes, A. A.. A benchmarking tool for the generation of bipartite network models with overlapping communities, Knowledge and Information Systems.

6. Valejo, A. and Faleiros, T. and Oliveira, M. C. F. and Lopes, A. A.. A coarsening method for labeled and unlabelled bipartite network via weight-constrainedlabel propagation, Knowledge-based Systems (KNOSYS), submitted paper. 
7. Minatel, D. and Valejo, A. and Lopes, A. A., Trajectory network assessment based on analysis of stay points cluster, 2018, The Brazilian Conference on Intelligent Systems (BRACIS).

8. Diego Minatel and Vinícius Ferreira and Alneu Lopes. A multilevel approach for building location-based social network by using stay points, 2019, The Brazilian Conference on Intelligent Systems (BRACIS), accepted paper

9. Ferreira, V. and Valejo, A. and Lopes, A. A.. A scalability approach based on multilevel optimization for link prediction methods, 2019, The Brazilian Conference on Intelligent Systems (BRACIS), accepted paper.

10. Cintra, D. S., Valejo, A. and Lopes, A. A. and Oliveira, M. C. F.. Visualization to Assist Interpretation of the Multilevel Paradigm in Bipartite Graphs, 2019, International Joint Conference on Computer Vision, Imaging and Computer Graphics Theory and Applications, submitted paper.

11. Fabbri, R. and Valejo, A. and Lopes, A. A. and Oliveira, M. C. F.. Interactive Visualization of Large Bipartite Networks Assisted by Multilevel Strategies, 2019, International Joint Conference on Computer Vision, Imaging and Computer Graphics Theory and Applications, submitted paper.

Finally, the following lists briefly present publications not directly related to this research, but comprise additional research efforts in which the author of this thesis participated as coauthor:

1. Berton, L. and Faleiros, T. and Valejo, A. and Valverde-rebaza, J. and Lopes, A. A.. Rgcli: robust graph that considers labeled instances for semi-supervised learning, 2016, Neurocomputing.

2. Gonçalves, V. P. and Costa, E. P. and Valejo, A. and Filho, G. P. R. and Johnson, T. M. and Pessin, G. and Ueyama, J.. Enhancing intelligence in multimodal emotion assessments, 2016, Applied intelligence.

3. Filho, G. P. R. and Villas, L. A. and Freitas, H. and Valejo, A. and Guidoni, D. L. and Ueyama, J.. ResiDI: Towards a smarter smart home system for decision-making using wireless sensors and actuators, 2018, Computer Networks.

4. Filho, G. P. R. and Neto, J. R. T. and Valejo, A. and Meneguette, R. I. and Villas, L. A.. and Ueyama, J.. Um Sistema de Controle Neuro-Fog para Infraestruturas Residenciais via Objetos Inteligentes, 2018, Simpósio Brasileiro de Redes de Computadores (SBRC).

5. Chuerubim, M. L. and Valejo, A. and Silva, I.. Spatial and temporal identification of community structures of road accidents through homogeneous complex networks and 
measures of centrality, 2018, Congresso Luso-Brasileiro para o Planeamento Urbano, Regional, Integrado e Sustentável (PLURIS).

6. Chuerubim, M. L. and Valejo, A. and Silva, I.. Bayesian networks modeling in traffic accidents: case study for brazil, 2018, Congresso de Ensino e Pesquisa em Transportes (ANPET).

7. Filho, G. P. R. and Mano, L. Y. and Valejo, A. and Villas, L. A. and Ueyama, J.. A lowcost smart home automation to enhance decision-making based on fog computing and computational intelligence, 2018, IEEE Latin America Transactions.

8. Faleiros, T., Valejo, A. and Lopes, A. A.. Unsupervised learning of textual pattern based on propagation in bipartite graph, 2019, Intelligent Data Analysis, accepted paper.

9. Chuerubim, M. L. and Bezerra, B. S. and Valejo, A. and Silva, I.. Limitation of classification tree models in investigating road accident severity, 2019, Revista de Engenharia Civil IMED, accepted paper.

10. Ferreira, V. and Valejo, A. and Valdivia, P. and Valverde-rebaza, J.. Exploiting Geographical Data to improve Recommender Systems for Business Opportunitiesin Urban Areas, 2019, The Brazilian Conference on Intelligent Systems (BRACIS), accepated paper.

11. Chuerubim, Maria Lígia and Valejo, A. and Bezerra, Barbara Stolte and Silva, Irineu, Artificial neural networks restriction for road accidents severity classification in unbalanced database, in Sigma Journal of Engineering and Natural Sciences, 2019

\subsection{Limitations and possible research directions}

Even though the proposed multilevel methods reduce the computational cost of solving complex optimization problems and can process large-scale bipartite networks, they implement a sequential (or batch mode) paradigm and with limited resources and therefore are still unfeasible on networks with hundreds of millions, or billions vertices and edges. In contrast, massive networks are intimately tied to the parallel and distributed paradigm, since even near-linear algorithms may require high processing times. To overcome these limitations the proposed algorithms could be associated with an on-demand strategy, in which a sub-network is locally built and processed as a part of the original data (commonly stored in a database). In general, these sub-networks encompass a specific content, which may not exceed a few hundreds of thousands vertices and edges. On-demand strategies are commonly employed in image processing or visualization scenarios, in which details are offered to users according to their demand or interaction actions, and unnecessary information can be hidden or released from memory (HAM; PERER, 2009).

Regarding technical limitations, identifying the level of coarsening that will yield a suitable trade-off between accuracy and execution times is a critical issue in applying the proposed 
multilevel method. Although the $M L P b_{C S V}$ minimizes this limitation, e.g. in a visualization scenario, choosing the best number of vertices in partitioning or community detection problems remains an open problem. Currently, this is done by means of empirical investigation in each application problem and dataset, but it certainly deserves further investigation.

Our algorithms can support the application of the multilevel method in bipartite networks and foster novel approaches to solving other problems beyond those addressed in this thesis. Accordingly, as this study applied a broad analytical methodology focused on the applicability of the method, a natural future research line is in-depth exploration of the described applications on specific domains, e.g. community detection in user-location (geo-referenced) networks or visualization of biological brain networks.

We are also particularly interested in applying the method in semi-supervised contexts, mainly in classification tasks. As $M L P b$ is a label propagation strategy it is straightforward to explore its potential in this scenario. The input labels can guide the coarsening process to build a more representative or faithful reduced version of the original topology, e.g. assisting visualization or network drawing tasks. Furthermore, the proposed method could speed up highcost and transductive classification algorithms. As discussed, the proposed coarsening strategies may be useful in the context of network visualization, and we plan to investigate the methods for interactive visualization of large networks. Also, extending the algorithms to handle problems defined in heterogeneous networks seems to be a promising pathway, since such networks are common in network science and applications (SHI et al., 2017a).

The potential of the proposed method was not exhaustively exploited. For instance, the ability to process layers independently was directly tackled only in the problem of dimensionality reduction. However, usage of different coarsening algorithms (or distinct parameterization of the same algorithm) in each layer offers a large space for further scientific exploration. 
ABELLO, J.; HAM, F. V.; KRISHNAN, N. Ask-graphview: A large scale graph visualization system. IEEE Transactions on Visualization and Computer Graphics, v. 12, n. 5, p. 669-676, 2006. Citations on pages 41 and 187.

ABOU-RJEILI, A.; KARYPIS, G. Multilevel algorithms for partitioning power-law graphs. In: Proceedings of the International Parallel and Distributed Processing Symposium (IPDPS). [S.1.: s.n.], 2006. p. 124-124. Citations on pages 41, 49, 56, 116, and 134.

AHN, Y.-Y.; BAGROW, J. P.; LEHMANN, S. Link communities reveal multiscale complexity in networks. Nature, v. 466, n. 7307, p. 761-764, 2010. Citation on page 102.

AKHREMTSEV, Y.; SANDERS, P.; SCHULZ, C. (semi-)external algorithms for graph partitioning and clustering. In: Proceedings of the Meeting on Algorithm Engineering \& Expermiments. [S.1.: s.n.], 2015. (ALENEX'15), p. 33-43. Citation on page 58.

AKOGLU, L. Quantifying political polarity based on bipartite opinion networks. In: Proceedings of the International AAAI Conference on Web and Social Media (AAAI), Eighth International AAAI Conference on Weblogs and Social Media (ICWSM). [S.1.: s.n.], 2014. Citation on page 98.

AKOGLU, L.; FALOUTSOS, C. Rtg: A recursive realistic graph generator using random typing. Lecture Notes in Computer Science (including subseries Lecture Notes in Artificial Intelligence and Lecture Notes in Bioinformatics), v. 5781 LNAI, n. PART 1, p. 13-28, 2009. Citation on page 88 .

ALBERT, R.; JEONG, H.; BARABASI, A.-L. Diameter of the world-wide web. Nature, v. 401, p. 130-131, 09 1999. Citation on page 23.

ALESSANDRO, M.; VITTORIO, C. C. Leveraging the nonuniform PSO network model as a benchmark for performance evaluation in community detection and link prediction. New Journal of Physics, IOP Publishing, v. 20, n. 6, p. 063022, jun 2018. Citation on page 89.

ALI, A. M.; ALVARI, H.; HAJIBAGHERI, A.; LAKKARAJ, K.; SUKTHANKAR, G. Synthetic generators for cloning social network data. In: Proceedings of the International Conference on Social Informatics (Socinfo). [S.1.: s.n.], 2014. Citation on page 88.

ALVISI, S. A new procedure for optimal design of district metered areas based on the multilevel balancing and refinement algorithm. Water Resources Management, v. 29, n. 12, p. 4397-4409, 2015. Citation on page 41.

ALZAHRANI, T.; HORADAM, K. J. Community Detection in Bipartite Networks: Algorithms and Case studies. In: Complex Systems and Networks. [S.1.: s.n.], 2015. p. 25-50. Citation on page 116.

Community detection in bipartite networks: Algorithms and case studies. In: LU, J.; YU, X.; CHEN, G.; YU, W. (Ed.). Proceeding of the Complex Systems and Networks: Dynamics, Controls and Applications. [S.1.: s.n.], 2016. p. 25-50. Citation on page 184. 
Archambault, D.; Munzner, T.; Auber, D. Grouseflocks: Steerable exploration of graph hierarchy space. IEEE Transactions on Visualization and Computer Graphics, v. 14, n. 4, p. 900-913, 2008. Citations on pages 41 and 187.

ARENAS, A.; DUCH, J.; FERNáNDEZ, A.; GóMEZ, S. Size reduction of complex networks preserving modularity. New Journal of Physics, v. 9, n. 6, p. 176, 2007. Citations on pages 41 and 57.

ARLEO, A.; DIDIMO, W.; LIOTTA, G.; MONTECCHIANI, F. A distributed multilevel forcedirected algorithm. In: HU, Y.; NÖLLENBURG, M. (Ed.). Graph Drawing and Network Visualization. Cham: Springer International Publishing, 2018. p. 3-17. Citations on pages 25 and 41 .

ARMSTRONG, T. G.; PONNEKANTI, V.; BORTHAKUR, D.; CALLAGHAN, M. Linkbench : a database benchmark based on the facebook social graph. In: Proceedings of the International Conference on Management of Data (SIGMOD). [S.1.: s.n.], 2013. p. 1185 - 1196. Citation on page 88 .

ASRATIAN, A. S.; DENLEY, T. M. J.; HäGGKVIST, R. Bipartite graphs and their applications. New York, NY, USA: Cambridge University Press, 1998. Citations on pages 24, 34, 130, and 162.

BABAI, L. Some applications of graph contractions. Journal of graph theory, Wiley Online Library, v. 1, n. 2, p. 125-130, 1977. Citation on page 38.

BANERJEE, S.; JENAMANI, M.; PRATIHAR, D. K. Algorithms for projecting a bipartite network. In: IEEE. 2017 Tenth International Conference on Contemporary Computing (IC3). [S.1.], 2017. p. 1-3. Citation on page 26.

Properties of a projected network of a bipartite network. In: IEEE. 2017 International Conference on Communication and Signal Processing (ICCSP). [S.1.], 2017. p. 0143-0147. Citations on pages 26 and 166.

BANOS, R.; GIL, C.; ORTEGA, J.; MONTOYA, F. G. Parallel heuristic search in multilevel graph partitioning. In: Proceedings of the Euromicro Conference on Parallel, Distributed and Network-Based Processing. [S.1.: s.n.], 2004. p. 88 - 95. Citation on page 116.

BAÑOS, R.; GIL, C.; ORTEGA, J.; MONTOYA, F. G. A parallel multilevel metaheuristic for graph partitioning. Journal of Heuristics, v. 10, n. 3, p. 315-336, 2004. Citations on pages 116 and 135 .

BAR-YAM, Y. General features of complex systems. Encyclopedia of Life Support Systems (EOLSS), UNESCO, EOLSS Publishers, Oxford, UK, p. 1, 2002. Citation on page 23.

BARABASI, A. L.; BONABEAU, E. Scale-free netwroks. Scientific American (SCIAM), 2003. Citation on page 88 .

BARBER, M. J. Modularity and community detection in bipartite networks. Physical Review E - Statistical, Nonlinear, and Soft Matter Physics, v. 76, n. 6, p. 1-11, 2007. Citations on pages 89 and 146 .

BARBER, M. J.; CLARK, J. W. Detecting network communities by propagating labels under constraints. Phys. Rev. E, v. 80, p. 026129, 2009. Citation on page 183. 
BARNARD, S. T. Pmrsb: Parallel multilevel recursive spectral bisection. In: Proceedings of the ACM/IEEE conference on Supercomputing (CDROM). [S.1.: s.n.], 1995. p. 1-27. Citations on pages 38,41 , and 135 .

BARNARD, S. T.; SIMON, H. D. Fast multilevel implementation of recursive spectral bisection for partitioning unstructured problems. Concurrency and Computation: Practice and Experience, v. 6, n. 2, p. 101-117, 1994. Citations on pages 38, 41, and 134.

BARRETT, C. L.; BECKMAN, R. J.; KHAN, M.; KUMAR, V. S. A.; MARATHE, M. V.; STRETZ, P. E.; DUTTA, T.; LEWIS, B. Generation and analysis of large synthetic social contact networks. In: Proceedings of the Winter Simulation Conference. [S.1.: s.n.], 2009. (WSC '09), p. 1003-1014. Citation on page 88.

BATAGELJ, V.; BRANDENBURG, F. J.; DIDIMO, W.; LIOTTA, G.; PALLADINO, P.; PATRIGNANI, M. Visual analysis of large graphs using (x,y)-clustering and hybrid visualizations. IEEE Transactions on Visualization and Computer Graphics, v. 17, n. 11, p. 1587-1598, 2011. Citations on pages 41 and 187.

BECKETT, S. J. Improved community detection in weighted bipartite networks. Royal society open science, v. 3, n. 1, p. 140536, 2016. ISSN 2054-5703. Citations on pages 89, 116, 122, and 147.

BENLIC, U.; HAO, J. K. A mltilevel memetic approach for improving graph k-partitions. IEEE Transactions on Evolutionary Computation, v. 15, n. 5, p. 624-642, 2011. Citation on page 39.

BICHOT, C.-E. Graph partitioning. In: [S.1.]: ISTE - Wiley, 2014. chap. A Partitioning requiring rapidity and quality: The multilevel method and partitions refinement algorithms, $\mathrm{p}$. 27-63. Citations on pages 130 and 135.

BIRMELÉ, E. A scale-free graph model based on bipartite graphs. Discrete Applied Mathematics, v. 157, n. 10, p. 2267 - 2284, 2009. Citation on page 88.

BLONDEL, V. D.; GUILLAUME, J.-L.; LAMBIOTTE, R.; LEFEBVRE, E. Fast unfolding of communities in large networks. Journal of Statistical Mechanics: Theory and Experiment, v. 10008 , n. 10, p. 6, 2008. Citations on pages 39, 57, and 183.

BLUM, C.; VALLES, M. Y. Multi-level ant colony optimization for dna sequencing by hybridization. In: SPRINGER. Proceedings of the International Workshop on Hybrid Metaheuristics. [S.1.], 2006. p. 94-109. Citation on page 135.

BONCZ, P. Ldbc: Benchmarks for graph and rdf data management. In: Proceedings of the International Database Engineering \& Applications Symposium. [S.1.: s.n.], 2013. p. 1-2. Citation on page 88.

BORNHOLDT, S.; SCHUSTER, H. G. (Ed.). Handbook of Graphs and Networks: From the Genome to the Internet. New York, NY, USA: John Wiley \& Sons, Inc., 2003. ISBN 3527403361. Citation on page 23.

BOUHMALA, N.; CAI, X. Partition of unstructured finite element meshes by a multilevel approach. In: Proceedings of the International Workshop on Applied Parallel Computing. [S.1.: s.n.], 2001. v. 1947, p. 187-195. Citation on page 53. 
BRANDT, A. Multilevel computations: Review and recent developments. In: Multigrid methods: Theory, applications, and supercomputing, proceedings of the 3rd Copper Mountain conference on multigrid methods. Lecture Notes in Pure and Appl Math. [S.l.: s.n.], 1988. v. 110 , p. 35-62. Citations on pages 38 and 130.

BRANNICK, J.; CHEN, Y.; KRAUS, J.; ZIKATANOV, L. Algebraic multilevel preconditioners for the graph laplacian based on matching in graphs. SIAM Journal on Numerical Analysis, v. 51, n. 3, p. 1805-1827, 2013. Citations on pages 55 and 77.

BUNIMOVICH, L.; WEBB, B. Isospectral graph reductions and improved estimates of matrices' spectra. Linear Algebra and its Applications, Elsevier, v. 437, n. 7, p. 1429-1457, 2012. Citation on page 70 .

BURGOS, E.; CEVA, H.; HERNÁNDEZ, L.; PERAZZO, R. P. J.; DEVOTO, M.; MEDAN, D. Two classes of bipartite networks: Nested biological and social systems. Phys. Rev. E, American Physical Society, v. 78, p. 046113, 2008. Citation on page 71.

CAI, D.; HE, X.; HAN, J.; HUANG, T. S. Graph regularized nonnegative matrix factorization for data representation. IEEE Transactions on Pattern Analysis and Machine Intelligence, v. 33, n. 8, p. 1548-1560, Aug 2011. Citation on page 77.

CANN, T. J. B.; WEAVER, I. S.; WILLIAMS, H. T. P. Is it correct to project and detect? assessing performance of community detection on unipartite projections of bipartite networks. In: Complex Networks and Their Applications VII. [S.l.]: Springer International Publishing, 2019. p. 267-279. Citation on page 184.

CAPOTA, M.; HEGEMAN, T.; IOSUP, A.; PRAT-PÉREZ, A.; ERLING, O.; BONCZ, P. Graphalytics: A big data benchmark for graph-processing platforms. In: Proceedings of the Graph Data management Experiences and Systems (GRADES). [S.1.: s.n.], 2015. p. 1-6. ISBN 9781450336116. Citation on page 88.

CHAKRABARTI, D.; ZHAN, Y.; FALOUTSOS, C. R-mat: A recursive model for graph mining. In: Proceedings of the Society for Industrial and Applied Mathematics (SIAM), International Conference on Data Mining (SDM). [S.1.: s.n.], 2004. p. 5. Citations on pages 88 and 89 .

CHAKRABORTY, T.; DALMIA, A.; MUKHERJEE, A.; GANGULY, N. Metrics for community analysis: A survey. ACM Comput. Surv., v. 50, n. 4, p. 54:1-54:37, 2017. Citation on page 184.

CHEN, H.; PEROZZI, B.; HU, Y.; SKIENA, S. Harp: Hierarchical representation learning for networks. In: Proceedings of the Thirty-Second AAAI Conference on Artificial Intelligence. [S.1.: s.n.], 2018. Citation on page 55.

CHEN, J.; SAFRO, I. Algebraic distance on graphs. SIAM Journal on Scientific Computing, v. 33, n. 6, p. 3468-3490, 2011. Citations on pages 55 and 76.

CHENG-GEN, W.; ZHANG, B. H.; LI, P.; SHU, J.; CHENG, L. Y.; HAO, Z. G.; ZHI-QIAN, B. O. Power system islanding based on multilevel reduced graph partitioning algorithm. In: Proceedings of the Universities Power Engineering Conference. [S.1.: s.n.], 2008. p. 1 - 6. Citation on page 41. 
CHERNOSKUTOV, M.; INEICHEN, Y.; BEKAS, C. Heuristic algorithm for approximation betweenness centrality using graph coarsening. Procedia Computer Science, Elsevier Masson SAS, v. 66, p. 83-92, 2015. Citations on pages 25, 41, 57, 130, and 135.

CHESSA, A.; CRIMALDI, I.; RICCABONI, M.; TRAPIN, L. Cluster analysis of weighted bipartite networks: A new copula-based approach. PLOS ONE, Public Library of Science, v. 9, n. 10, p. 1-12, 10 2014. Citation on page 184.

CHEVALIER, C.; PELLEGRINI, F. Improvement of the efficiency of genetic algorithms for scalable parallel graph partitioning in a multi-level framework. In: Proceedings of the European Conference on Parallel Processing (Euro-Par). [S.1.: s.n.], 2006. v. 4128, p. 243-252. Citation on page 42.

CHEVALIER, C.; SAFRO, I. Comparison of coarsening schemes for multilevel graph partitioning. In: Proceedings of the International Conference on Learning and Intelligent Optimization (LION). [S.1.: s.n.], 2009. v. 5851 LNCS, p. 191-205. Citation on page 34.

CLAUSET, A.; NEWMAN, M. E. J.; MOORE, C. Finding community structure in very large networks. Phys. Rev. E, v. 70, p. 066111, 2004. Citations on pages 57 and 183.

CONG, J.; SMITH, M. A parallel bottom-up clustering algorithm with applications to circuit partitioning in vlsi design. In: Proceedings of the ACM/IEEE Design Automation Conference. [S.1.: s.n.], 1993. p. 755-760. Citation on page 38.

CORMEN, T. H.; LEISERSON, C. E.; RIVEST, R. L.; STEIN, C. Introduction to Algorithms, Third Edition. 3rd. ed. [S.1.]: The MIT Press, 2009. ISBN 0262033844, 9780262033848. Citation on page 101.

CRAWFORD, C.; WALSHAW, C.; SOPER, A. A multilevel force-directed graph drawing algorithm using multilevel global force approximation. In: Proceedings of the International Conference on Information Visualisation. [S.1.: s.n.], 2012. p. 454-459. Citation on page 41.

CUI, Y.; WANG, X. Uncovering overlapping community structures by the key bi-community and intimate degree in bipartite networks. Physica A: Statistical Mechanics and its Applications, v. 407 , n. 0 , p. 7-14, 2014. Citation on page 87.

DAI, C.; LI, P. C.; TOULOUSE, M. A cooperative multilevel tabu search algorithm for the covering design problem. In: Proceedings of the International Conference on Artificial Evolution. [S.1.]: Springer Berlin Heidelberg, 2006. v. 3871 LNCS, p. 119-130. Citation on page 135.

DANON, L.; DÍAZ-GUILERA, A.; DUCH, J.; ARENAS, A. Comparing community structure identification. Journal of Statistical Mechanics: Theory and Experiment, IOP Publishing, v. 2005, p. P09008, 2005. Citation on page 107.

DEMSAR, J. Statistical comparisons of classifiers over multiple data sets. The Journal of Machine Learning Research, v. 7, p. 1-30, 2006. Citations on pages 104, 123, 149, 158, 180, and 185 .

DIAS, M. D.; MANSOUR, M. R.; DIAS, F.; PETRONETTO, F.; SILVA, C. T.; NONATO, L. G. A hierarchical network simplification via non-negative matrix factorization. In: Proceedings of the Conference on Graphics, Patterns and Images (SIBGRAPI). [S.1.: s.n.], 2017. p. 119126. Citations on pages 41, 55, 78, and 187 . 
DÍAZ, J.; PETIT, J.; SERNA, M. A survey of graph layout problems. ACM Comput. Survey, ACM, v. 34, n. 3, p. 313-356, 2000. Citation on page 187.

DJIDJEV, H. N. A scalable multilevel algorithm for graph clustering and community structure detection. In: Proceedings of the International Workshop on Algorithms and Models for the Web-Graph (WAW). [S.1.: s.n.], 2008. p. 117-128. Citations on pages 116 and 135.

DJIDJEV, H. N.; ONUS, M. Scalable and accurate graph clustering and community structure detection. IEEE Transactions on Parallel and Distributed Systems, v. 24, n. 5, p. 1022-1029, 2013. Citations on pages 116 and 135.

DORMANN, C. f.; STRAUSS, R. Detecting modules in quantitative bipartite networks: the QuaBiMo algorithm. arXiv preprint, v. 1304.3218, 2013. Citation on page 116.

DORMANN, C. F.; STRAUSS, R. A method for detecting modules in quantitative bipartite networks. Methods in Ecology and Evolution, v. 5, n. 1, p. 90-98, 2014. Citation on page 116.

DRAKE, D. E.; HOUGARDY, S. Linear time local improvements for weighted matchings in graphs. In: Experimental and Efficient Algorithms. [S.1.: s.n.], 2003. p. 107-119. Citation on page 54 .

A simple approximation algorithm for the weighted matching problem. Information Processing Letters, v. 85, n. 4, p. 211-213, 2003. Citation on page 54.

DU, N.; WANG, B.; WU, B.; WANG, Y. Overlapping community detection in bipartite networks. Proceedings of the International Conference on Web Intelligence (IEEE/WIC/ACM), n. 60402011, p. 176-179, 2008. Citation on page 87.

DUCOURNAU, A.; RITAL, S.; BRETTO, A.; LAGET, B. A multilevel spectral hypergraph partitioning approach for color image segmentation. In: Proceedings of the IEEE International Conference on Signal and Image Processing Applications, Conference Proceedings (ICSIPA). [S.1.: s.n.], 2009. p. 419-424. Citation on page 41.

EADES, P. A heuristic for graph drawing. Congressus Numerantium, v. 42, p. 149-160, 1984. Citation on page 188 .

EPPSTEIN, D. Sparsification - a technique for speeding up dynamic graph algorithms. In: Proceedings of the Annual Symposium on Foundations of Computer Science. [S.1.: s.n.], 1992. p. 60-69. Citation on page 38.

ERCIYE, K.; ALP, A.; MARSHALL, G. Serial and parallel multilevel graph partitioning using fixed centers. In: Proceedings of the International Conference on Current Trends in Theory and Practice of Computer Science (SOFSEM). [S.1.: s.n.], 2005. p. 127-136. Citations on pages 54,116 , and 135 .

FALEIROS, T. d. P.; ROSSI, R. G.; LOPES, A. A. Optimizing the class information divergence for transductive classification of texts using propagation in bipartite graphs. Pattern Recognition Letters, v. 87, p. 127 - 138, 2017. Citations on pages 24, 71, 87, 95, 98, and 162.

FALOUTSOS, M.; FALOUTSOS, P.; FALOUTSOS, C. On power-law relationships of the internet topology. In: Proceedings of the Conference on Applications, Technologies, Architectures, and Protocols for Computer Communication. [S.1.: s.n.], 1999. (SIGCOMM '99), p. 251-262. Citation on page 23 . 
FANG, H.-R.; SAAD, Y. Hypergraph-based multilevel matrix approximation for text information retrieval. In: Proceedings of the ACM international conference on Information and knowledge management (CIKM). [S.1.: s.n.], 2010. p. 1597. Citation on page 41.

FANG, H.-R.; SAKELLARIDI, S.; SAAD, Y. Multilevel manifold learning with application to spectral clustering. In: Proceedings of the ACM international conference on Information and knowledge management (CIKM). [S.1.: s.n.], 2010. p. 419. Citations on pages 41 and 68.

FEDER, T.; MOTWANI, R. Clique partitions, graph compression and speeding-up algorithms. Journal of Computer and System Sciences, Elsevier, v. 51, n. 2, p. 261-272, 1995. Citation on page 57.

FIDUCCIA, C. M.; MATTHEYSES, R. M. A linear-time heuristic for improving network partitions. In: Proceedings of the Design Automation Conference. Piscataway, NJ, USA: [s.n.], 1982. (DAC' '82), p. 175-181. Citation on page 64.

FORTUNATO, S. Community detection in graphs. Physics Reports, v. 486, n. 3-5, p. 75-174, 2010. Citations on pages 25, 66, 86, 91, 92, 116, 146, and 184.

FRISHMAN, Y.; TAL, A. Multi-level graph layout on the gpu. IEEE Transactions on Visualization and Computer Graphics, v. 13, n. 6, p. 1310-1317, 2007. Citation on page 41.

FRUCHTERMAN, T. M. J.; REINGOLD, E. M. Graph drawing by force-directed placement. Softw. Pract. Exper., John Wiley \& Sons, Inc., v. 21, n. 11, p. 1129-1164, 1991. Citations on pages 67,107 , and 188 .

FUNKE, S. Topological hole detection in wireless sensor networks and its applications. In: Proceedings of the 2005 Joint Workshop on Foundations of Mobile Computing. [S.1.: s.n.], 2005. (DIALM-POMC '05), p. 44-53. Citation on page 193.

GABOW, H. N.; GALIL, Z.; SPENCER, T. H. Efficient implementation of graph algorithms using contraction. Journal of the ACM (JACM), ACM, v. 36, n. 3, p. 540-572, 1989. Citation on page 38 .

GALAN, S. F.; MENGSHOEL, O. J. Neighborhood beautification: Graph layout through message passing. Journal of Visual Languages \& Computing, v. 44, p. 72 - 88, 2018. Citation on page 41 .

GANSNER, E. R.; HU, Y.; NORTH, S.; SCHEIDEGGER, C. Multilevel agglomerative edge bundling for visualizing large graphs. In: Proceedings of the IEEE Pacific Visualization Symposium (PacificVis). [S.1.: s.n.], 2011. p. 187-194. Citation on page 41.

GEBREMEDHIN, A. H.; MANNE, F.; POTHEN, A. Graph coloring in optimization revisited. [S.1.]: Department of Informatics, University of Bergen, 2002. (Reports in informatics). Citation on page 164.

Parallel distance-k coloring algorithms for numerical optimization. In: Proceedings of the 8th International Euro-Par Conference on Parallel Processing. [S.1.: s.n.], 2002. (Euro-Par '02), p. 912-921. Citation on page 164. 
GHONIEM, M.; FEKETE, J.-D.; CASTAGLIOLA, P. On the readability of graphs using nodelink and matrix-based representations: A controlled experiment and statistical analysis. Information Visualization, Palgrave Macmillan, v. 4, n. 2, p. 114-135, 2005. Citations on pages 187, 190, and 194.

GIRVAN, M.; NEWMAN, M. E. J. Community structure in social and biological networks. In: Proceedings of the National Academy of Science of the United States of America. [S.1.: s.n.], 2002. v. 99, p. 7821-7826. Citations on pages 88 and 116.

GLANTZ, R.; MEYERHENKE, H.; SCHULZ, C. Tree-based coarsening and partitioning of complex networks. In: Proceedings of the International Symposium on Experimental Algorithms (SEA). [S.1.: s.n.], 2014. v. 8504 LNCS, p. 364-375. Citations on pages 55 and 77.

GRUJIĆ, J. Movies recommendation networks as bipartite graphs. In: Proceedings of the International Conference on Computational Science (ICCS). [S.1.]: Springer Berlin Heidelberg, 2008. p. 576-583. Citations on pages 24, 87, and 162.

GUPTA, A. Fast and effective algorithms for graph partitioning and sparse-matrix ordering. IBM Journal of Research and Development, IBM, v. 41, n. 1.2, p. 171-183, 1997. Citations on pages 58 and 67.

HACHUL, S.; JÜNGER, M. Drawing large graphs with a potential-field-based multilevel algorithm. In: Proceedings of the International Symposium on Graph Drawing (GD). [S.1.: s.n.], 2005. p. 285-295. Citation on page 41.

HACHUL, S.; JüNGER, M. Large-graph layout algorithms at work: An experimental study. J. Graph Algorithms Appl., v. 11, p. 345-369, 2007. Citation on page 187.

HAM, F. van; PERER, A. "search, show context, expand on demand": Supporting large graph exploration with degree-of-interest. IEEE Transactions on Visualization and Computer Graphics, IEEE Educational Activities Department, v. 15, n. 6, p. 953-960, 2009. Citation on page 201.

HAN, J.; LU, J.; HU, Y.; ZHANG, G. Tri-level decision-making with multiple followers: Model, algorithm and case study. Information Sciences, v. 311, p. $182-204,2015$. Citation on page 130.

HENDRICKSON, B.; LELAND, R. A multilevel algorithm for partitioning graphs. In: Proceedings of the ACM/IEEE Conference on Supercomputing. [S.1.: s.n.], 1995. p. 28. Citations on pages $38,39,41,42$, and 64 .

HEUER, T.; SCHLAG, S. Improving coarsening schemes for hypergraph partitioning by exploiting community structure. In: Proceedings of the International Symposium on Experimental Algorithms (SEA 2017). Dagstuhl, Germany: [s.n.], 2017. (Leibniz International Proceedings in Informatics (LIPIcs), v. 75), p. 21:1-21:19. Citation on page 41.

HOLTGREWE, M.; SANDERS, P.; SCHULZ, C. Engineering a scalable high quality graph partitioner. In: Proceedings of the IEEE International Symposium on Parallel Distributed Processing (IPDPS). [S.1.: s.n.], 2010. p. 1-12. Citations on pages 55 and 75.

HUANG, J.; SUN, H.; HAN, J.; DENG, H.; SUN, Y.; LIU, Y. Shrink: A structural clustering algorithm for detecting hierarchical communities in networks. In: Proceedings of the International Conference on Information and Knowledge Management. [S.1.: s.n.], 2010. p. 219-228. Citation on page 57. 
HUANG, X.; LAI, W.; ZHANG, D.; HUANG, M.; NGUYEN, Q. V. A kernel-based algorithm for multilevel drawing web graphs. In: Proceedings of the Computer Graphics, Imaging and Visualisation (CGIV). [S.1.: s.n.], 2007. p. 454-459. Citation on page 41.

HWANG, T.; SICOTTE, H.; TIAN, Z.; WU, B.; KOCHER, J.-P.; WIGLE, D. A.; KUMAR, V.; KUANG, R. Robust and efficient identification of biomarkers by classifying features on graphs. Bioinformatics, v. 24, n. 18, p. 2023-2029, 2008. Citations on pages 24, 87, 95, and 162.

JEONG, H.; TOMBOR, B.; ALBERT, R.; OLTVAI, Z. N.; BARABASI, A. L. The large-scale organization of metabolic networks. Nature, v. 407, n. 6804, p. 651-654, 2000. Citations on pages $23,24,115$, and 129 .

JIAN-BIN, C.; XIANG-JUN, D.; HAN-TAO, S. The refinement algorithm consideration in text clustering scheme based on multilevel graph. Wuhan University Journal of Natural Sciences, v. 9 , n. 5 , p. 671-675, 2004. Citation on page 41 .

JOLLIFFE, I. Principal component analysis. [S.1.]: Springer, 2002. (Springer Series in Statistics). Citation on page 154.

JONNALAGADDA, A.; KUPPUSAMY, L. A survey on game theoretic models for community detection in social networks. Social Network Analysis and Mining, v. 6, n. 1, p. 83, 2016. Citation on page 86.

KAMADA, T.; KAWAI, S. An algorithm for drawing general undirected graphs. Inf. Process. Lett., v. 31, n. 1, p. 7-15, 1989. ISSN 0020-0190. Citation on page 188.

KANG, Y.; GU, X.; WANG, W.; MENG, D. Scalable clustering algorithm via a triangle folding processing for complex networks. In: Proceedings of the ACM International on Conference on Information and Knowledge Management (CIKM). [S.1.: s.n.], 2015. p. 33-42. Citations on pages 58 and 67.

KANG, Y.; YU, B.; WANG, W.; MENG, D. Spectral clustering for large-scale social networks via a pre-coarsening sampling based nystrom method. In: Proceedings of the Pacific-Asia Conference on Knowledge Discovery and Data Mining (PAKDD). [S.1.: s.n.], 2015. v. 9078, p. 106-118. Citations on pages 58 and 67.

KARGER, D. R.; STEIN, C. An Õ(n2) algorithm for minimum cuts. In: Proceedings of the Twenty-fifth Annual ACM Symposium on Theory of Computing. New York, NY, USA: ACM, 1993. (STOC '93), p. 757-765. ISBN 0-89791-591-7. Available: <http://doi.acm.org/10. 1145/167088.167281>. Citation on page 60.

KARPINSKI, M.; RYTTER, W. Fast parallel algorithms for graph matching problems. [S.1.]: Oxford University Press, 1998. Citation on page 142.

KARYPIS, G.; AGGARWAL, R.; KUMAR, V.; SHEKHAR, S. Multilevel hypergraph partitioning: Application in vlsi domain. In: Proceedings of the ACM/IEEE Design Automation Conference. [S.1.: s.n.], 1997. v. 7, n. 1, p. 69-79. Citations on pages 39, 41, and 61.

KARYPIS, G.; KUMAR, V. Analysis of multilevel graph partitioning. In: Proceedings of the ACM/IEEE Conference on Supercomputing. [S.1.: s.n.], 1995. p. 1-19. Citations on pages 24, 34, 38, 41, 130, and 134.

METIS - Unstructured Graph Partinioning and Sparse Matrix Ordering System.

[S.1.], 1995. 1-16 p. Citations on pages 38, 42, 52, and 53. 
Parallel multilevel graph partitioning. In: Proceedings of International Conference on Parallel Processing. [S.1.: s.n.], 1996. p. 314-319. Citations on pages 41 and 135.

. A fast and high quality multilevel scheme for partitioning irregular graphs. SIAM Journal on Scientific Computing, v. 20, n. 1, p. 359-392, 1998. Citations on pages 39, 41, 44, 52, 53, $119,121,122,133,134$, and 166.

Multilevel k-way partitioning scheme for irregular graphs. Journal of Parallel and Distributed Computing, v. 48, n. 1, p. 96-129, 1998. Citations on pages 39, 41, 52, and 64.

A parallel algorithm for multilevel graph partitioning and sparse matrix ordering. Journal of Parallel and Distributed Computing, v. 48, n. 1, p. 71-95, 1998. Citation on page 135.

Multilevel k-way hypergraph partitioning. In: Proceedings of the ACM/IEEE conference on Design automation conference (DAC). [S.1.: s.n.], 1999. p. 343-348. Citation on page 41.

Parallel multilevel k-way partitioning scheme for irregular graphs. In: Proceedings of the ACM/IEEE Conference on Supercomputing (CS). [S.1.: s.n.], 1999. v. 41, n. 2, p. 278-300. Citations on pages 41 and 135 .

KERNIGHAN, B. W.; LIN, S. An efficient heuristic procedure for partitioning graphs. Bell System Technical Journal, v. 49, n. 2, p. 291-307, 1970. Citation on page 64.

KHAN, A.; LI, N.; YAN, X.; GUAN, Z.; CHAKRABORTY, S.; TAO, S. Neighborhood based fast graph search in large networks. In: Proceedings of the International Conference on Management of Data (SIGMOD). [S.1.: s.n.], 2011. (SIGMOD '11), p. 901-912. Citation on page 142 .

KHOKHER, M. R.; GHAFOOR, A.; SIDDIQUI, A. M. Multilevel graph cuts based image segmentation. In: Proceedings of the International Conference on Digital Image Computing Techniques and Applications (DICTA). [S.1.: s.n.], 2012. p. 1-8. Citation on page 41.

KITCHENHAM, B.; PRETORIUS, R.; BUDGEN, D.; BRERETON, O. P.; TURNER, M.; M., N.; LINKMAN, S. Systematic literature reviews in software engineering - a tertiary study. Information and Software Technology, v. 52, n. 8, p. $792-805,2010$. Citation on page 35.

KITSAK, M.; KRIOUKOV, D. Hidden variables in bipartite networks. Physical Review E, APS, v. 84, n. 2, p. 026114, 2011. Citation on page 26.

KITSAK, M.; PAPADOPOULOS, F.; KRIOUKOV, D. Latent geometry of bipartite networks. Phys. Rev. E, American Physical Society, v. 95, p. 032309, Mar 2017. Available: <https://link. aps.org/doi/10.1103/PhysRevE.95.032309>. Citations on pages 23 and 26.

KOLLIAS, G.; SATHE, M.; SCHENK, O.; GRAMA, A. Fast parallel algorithms for graph similarity and matching. Journal of Parallel and Distributed Computing, v. 74, n. 5, p. 2400 - 2410, 2014. Citation on page 142.

KOROŠEC, P.; ŠILC, J.; ROBIČ, B. Mesh partitioning: A multilevel ant-colony-optimization algorithm. In: Proceedings of the International Parallel and Distributed Processing Symposium (IPDPS). [S.1.: s.n.], 2003. Citations on pages 41 and 134.

KUANG, D.; DING, C.; PARK, H. Symmetric nonnegative matrix factorization for graph clustering. In: Proceedings of the SIAM International Conference on Data Mining. [S.1.: s.n.], 2012. p. 106-117. Citation on page 78. 
KUNEGIS, J.; LUCA, E. W. D.; ALBAYRAK, S. The link prediction problem in bipartite networks. In: HÜLLERMEIER, E.; KRUSE, R.; HOFFMANN, F. (Ed.). Computational Intelligence for Knowledge-Based Systems Design. [S.1.]: Springer Berlin Heidelberg, 2010. p. 380-389. Citation on page 71.

LABATUT, V. Generalized Measures for the Evaluation of Community Detection Methods. CoRR, abs/1303.5, p. 44, 2013. Citations on pages 122, 147, and 179.

LANCICHINETTI, A.; FORTUNATO, S. Community detection algorithms: A comparative analysis. Physical Review E - Statistical, Nonlinear, and Soft Matter Physics, v. 80, n. 5, p. 1-11, 2009. Citations on pages 88 and 100.

LANCICHINETTI, A.; FORTUNATO, S.; RADICCHI, F. Benchmark graphs for testing community detection algorithms. Physical Review E - Statistical, Nonlinear, and Soft Matter Physics, v. 78, n. 4, p. 1-5, 2008. Citations on pages 86, 88, and 100.

LANCICHINETTI, A.; RADICCHI, F.; RAMASCO, J. J.; FORTUNATO, S. Finding statistically significant communities in networks. Plos One, v. 6, n. 4, p. 1-18, 04 2011. Citation on page 102.

LANDESBERGER, T. von; KUIJPER, A.; SCHRECK, T.; KOHLHAMMER, J.; WIJK, J. J. van; FEKETE, J.-D.; FELLNER, D. W. Visual analysis of large graphs: State-of-the-art and future research challenges. Computer Graphics Forum, v. 30, n. 6, p. 1719-1749, 2011. Citation on page 187.

LARGERON, C.; MOUGEL, P. N.; RABBANY, R.; ZAÏANE, O. R. Generating attributed networks with communities. Plos One, v. 10, n. 4, p. 1-21, 2015. ISSN 19326203. Citation on page 88 .

LARREMORE, D. B.; CLAUSET, A.; JACOBS, A. Z. Efficiently inferring community structure in bipartite networks. Phys. Rev. E, American Physical Society, v. 90, p. 012805, Jul 2014. Citations on pages 89, 105, 107, 108, 116, 163, and 178.

LASALLE, D.; KARYPIS, G. Multi-threaded graph partitioning. In: Proceedings of the IEEE International Parallel and Distributed Processing Symposium (IPDPS). [S.1.: s.n.], 2013. p. 225-236. Citations on pages 39, 41, 116, and 135.

Multi-threaded modularity based graph clustering using the multilevel paradigm. Journal of Parallel and Distributed Computing, v. 76, p. 66-80, 2015. Citations on pages 25, 41, 57, 116,130 , and 135.

LATAPY, M.; MAGNIEN, C.; VECCHIO, N. D. Basic notions for the analysis of large two-mode networks. Social Networks, v. 30, n. 1, p. 31 - 48, 2008. Citations on pages 23, 95, and 132.

LEHMANN, S.; SCHWARTZ, M.; HANSEN, L. K. Biclique communities. Physical Review E - Statistical, Nonlinear, and Soft Matter Physics, v. 78, n. 1, p. 1-9, 2008. Citation on page 87.

LI, Z.; ZHANG, S.; ZHANG, X. Mathematical model and algorithm for link community detection in bipartite networks. American Journal of Operations Research, v. 5, p. 421-434, 2015. Citation on page 87. 
LINDELAUF, R.; BORM, P.; HAMERS, H. One-mode projection analysis and design of covert affiliation networks. Social Networks, Elsevier, v. 34, n. 4, p. 614-622, 2012. Citation on page 26.

LIU, W.; LI, Z. An efficient parallel algorithm of n-hop neighborhoods on graphs in distributed environment. Frontiers of Computer Science, Springer, p. 1-17, 2018. Citation on page 26.

LIU, X.; LIU, W.; MURATA, T.; WAKITA, K. A framework for community detection in heterogeneous multi-relational networks. Advances in complex Systems, World Scientific, v. 17 , n. 06 , p. 1450018,2014 . Citation on page 40.

LIU, Y.; SAFAVI, T.; DIGHE, A.; KOUTRA, D. Graph summarization methods and applications: A survey. ACM Computing Surveys (CSUR), ACM, v. 51, n. 3, p. 62, 2018. Citations on pages 34 and 62 .

LOMBAERT, H.; SUN, Y.; GRADY, L.; XU, C. A multilevel banded graph cuts method for fast image segmentation. In: Proceedings of the International Conference on Computer Vision (ICCV). [S.1.: s.n.], 2005. Citation on page 41.

LU, J.; HAN, J.; HU, Y.; ZHANG, G. Multilevel decision-making: A survey. Information Sciences, v. 346-347, p. 463 - 487, 2016. Citation on page 130.

LÜ, L.; ZHOU, T. Link prediction in weighted networks : The role of weak ties. Europhysics Letters (EPL), v. 89, n. 1, p. 18001, 2010. Citations on pages 43, 132, and 164.

Lü, L.; ZHOU, T. Link prediction in complex networks: A survey. Physica A: Statistical Mechanics and its Applications, v. 390, n. 6, p. 1150 - 1170, 2011. ISSN 0378-4371. Citation on page 71 .

MA, J.; LI, S.; HAO, A.; QIN, H. Unsupervised co-segmentation of complex image set via bi-harmonic distance governed multi-level deformable graph clustering. In: Proceedings of the IEEE International Symposium on Multimedia (ISM). [S.1.: s.n.], 2013. p. 38-45. Citation on page 41 .

MA, X.; DONG, D.; WANG, Q. Community detection in multi-layer networks using joint nonnegative matrix factorization. IEEE Transactions on Knowledge and Data Engineering, IEEE, v. 31, n. 2, p. 273-286, 2018. Citation on page 71.

MA, X.; SUN, P.; QIN, G. Nonnegative matrix factorization algorithms for link prediction in temporal networks using graph communicability. Pattern Recognition, Elsevier, v. 71, p. 361-374, 2017. Citation on page 71.

MAHMOUD, H.; MASULLI, F.; ROVETTA, S.; RUSSO, G. Community detection in proteinprotein interaction networks using spectral and graph approaches. In: Computational Intelligence Methods for Bioinformatics and Biostatistics. [S.1.]: Springer International Publishing, 2014. p. 62-75. Citation on page 116.

MANSOUR, N.; FOX, G. C. Allocating data to multicomputer nodes by physical optimization algorithms for loosely synchronous computations. Concurrency and Computation: Practice and Experience, John Wiley and Sons Ltd., Chichester, UK, v. 4, n. 7, p. 557-574, Oct. 1992. ISSN 1040-3108. Citation on page 38. 
MANSOUR, N.; PONNUSAMY, R.; CHOUDHARY, A.; FOX, G. C. Graph contraction for physical optimization methods: A quality-cost tradeoff for mapping data on parallel computers. In: Proceedings of the International Conference on Supercomputing. New York, NY, USA: [s.n.], 1993. (ICS '93), p. 1-10. ISBN 0-89791-600-X. Citation on page 38.

MAO, G.; ZHANG, N. A multilevel simplification algorithm for computing the average shortestpath length of scale-free complex network. Journal of Applied Mathematics, v. 2014, 2014. Citation on page 41 .

MAUE, J.; SANDERS, P. Engineering algorithms for approximate weighted matching. In: Proceedings of the International Conference on Experimental Algorithms. Berlin, Heidelberg: Springer-Verlag, 2007. (WEA'07), p. 242-255. Citation on page 54.

MCDAID, A. F.; GREENE, D.; HURLEY, N. Normalized mutual information to evaluate overlapping community finding algorithms. In: eprint arXiv:1110.2515. [S.1.: s.n.], 2011. Citation on page 102 .

MELAMED, D. Community structures in bipartite networks: A dual-projection approach. Plos One, v. 9, n. 5, p. 1-5, 05 2014. Citations on pages 89, 102, 105, and 106.

MEYERHENKE, H.; NÖLLENBURG, M.; SCHULZ, C. Drawing large graphs by multilevel maxent-stress optimization. In: Proceedings of the International Symposium on Graph Drawing and Network Visualization. [S.1.: s.n.], 2015. v. 9411, p. 30-43. Citation on page 41.

MEYERHENKE, H.; NOLLENBURG, M.; SCHULZ, C. Drawing large graphs by multilevel maxent-stress optimization. IEEE Transactions on Visualization and Computer Graphics, PP, n. 99, p. 1-1, 2018. Citation on page 41.

MEYERHENKE, H.; SANDERS, P.; SCHULZ, C. Partitioning complex networks via sizeconstrained clustering. In: Proceedings of the International Symposium on Experimental Algorithms - Volume 8504. [S.1.]: Springer-Verlag New York, Inc., 2014. p. 351-363. Citations on pages $57,58,80$, and 81 .

MONIEN, B.; PREIS, R.; DIEKMANN, R. Quality matching and local improvement for multilevel graph-partitioning. Parallel Computing, v. 26, n. 12, p. 1609-1634, 2000. Citation on page 54.

MOUSSIADES, L.; VAKALI, A. Benchmark graphs for the evaluation of clustering algorithms. In: Proceedings of the International Conference on Research Challenges in Information Science (RCIS). [S.1.: s.n.], 2009. p. 197-206. Citation on page 88.

NETTLETON, D. F. A synthetic data generator for online social network graphs. Social Network Analysis and Mining, v. 6, n. 1, p. 44, 2016. Citation on page 88.

NEWMAN, M. E. J. Scientific collaboration networks: I. network construction and fundamental results. Physical Review E - Statistical, Nonlinear, and Soft Matter Physics, v. 64, n. 1, p. 1-8, 2001. Citations on pages 23, 24, 87, 95, 143, and 162 .

Scientific collaboration networks. ii. shortest paths, weighted networks, and centrality. Physical Review E - Statistical, Nonlinear, and Soft Matter Physics, v. 64, n. 1, p. 016132, 2001. Citations on pages 23, 24, 87, 143, and 162. 
The structure of scientific collaboration networks. Proceedings of the National Academy of Sciences of the United States of America, v. 98, n. 2, p. 404-409, 2001. ISSN 0027-8424. Citations on pages 118 and 130 .

Mixing patterns in networks. Physical Review E - Statistical, Nonlinear, and Soft Matter Physics, v. 67, n. 2, p. 026126, 2003. Citations on pages 23, 44, and 132.

. Modularity and community structure in networks. Proceedings of the National Academy of Sciences, v. 103, n. 23, p. 8577-8582, 2006. Citations on pages 64 and 135.

. Networks: An Introduction. New York, NY, USA: Oxford University Press, Inc., 2010. ISBN 0199206651, 9780199206650. Citations on pages 25 and 86.

NOACK, A.; ROTTA, R. Multi-level algorithms for modularity clustering. In: Proceedings of the International Symposium on Experimental Algorithms (SEA). [S.1.: s.n.], 2009. v. 5526 LNCS, p. 257-268. Citations on pages 24, 39, 41, 57, 64, 67, 70, 79, 80, 116, 130, and 135.

ODUNTAN, I. O. A multilevel search algorithm for feature selection in biomedical data. 91 p. Phd Thesis (PhD Thesis) - Dept. Computer Science, University of manitoba, 2005. Citation on page 135.

OLIVEIRA, S.; SEOK, S. C. A multi-level approach for document clustering. In: Proceedings of the International Conference on Computational Science (ICCS). [S.1.: s.n.], 2005. v. 3514, p. 204-211. Citation on page 41.

A multilevel approach to identify functional modules in a yeast protein-protein interaction network. In: Proceedings of the International Conference on Computational Science (ICCS). [S.1.: s.n.], 2006. p. 726-733. Citations on pages 41 and 134.

Multilevel approaches for large-scale proteomic networks. International Journal of Computer Mathematics, v. 84, n. 5, p. 683-695, 2007. Citations on pages 41, 58, and 67.

. Triangular clique based multilevel approaches to identify protein functional modules. In: DAYDÉ, M.; PALMA, J. M. L. M.; COUTINHO, Á. L. G. A.; PACITTI, E.; LOPES, J. C. (Ed.). Proceedings of the High Performance Computing for Computational Science (VECPAR). [S.l.]: Springer Berlin Heidelberg, 2007. p. 556-565. Citation on page 41.

OLIVEIRA, S.; SEOK, S.-C. A matrix-based multilevel approach to identify functional protein modules. International journal of bioinformatics research and applications, v. 4, n. 1, p. 11-27, 2008. Citation on page 41.

ON, B.; LEE, D. Scalable name disambiguation using multi-level graph partition. In: Proceedings of the SIAM international conference on data mining (SDM). [S.1.: s.n.], 2007. p. 575-580. Citation on page 41.

OPSAHL, T. Triadic closure in two-mode networks: Redefining the global and local clustering coefficients. jun 2010. Citation on page 118.

OSEI-KUFFUOR, D.; LI, R.; SAAD, Y. Matrix reordering using multilevel graph coarsening for ilu preconditioning. SIAM Journal on Scientific Computing, v. 37, n. 1, p. A391-A419, 2015. Citation on page 41 . 
PADRóN, B.; NOGALES, M.; TRAVESET, A. Alternative approaches of transforming bimodal into unimodal mutualistic networks. the usefulness of preserving weighted information. Basic and Applied Ecology, v. 12, n. 8, p. 713 - 721, 2011. ISSN 1439-1791. Citation on page 118.

PAREJO, J. A.; RUIZ-CORTÉS, A.; LOZANO, S.; FERNANDEZ, P. Metaheuristic optimization frameworks: a survey and benchmarking. Soft Computing, Springer, v. 16, n. 3, p. 527-561, 2012. Citation on page 27.

PASTA, M. Q.; ZAIDI, F. Leveraging evolution dynamics to generate benchmark complex networks with community structures. In: eprint arXiv:1606.01169. [S.1.: s.n.], 2016. abs/1606.01169. Citation on page 88 .

PELLEGRINI, F.; ROMAN, J. Scotch: A software package for static mapping by dual recursive bipartitioning of process and architecture graphs. In: Proceedings of the International Conference and Exhibition on High-Performance Computing and Networking. [S.l.: s.n.], 1996. (HPCN Europe 1996), p. 493-498. Citations on pages 41 and 42.

PÉREZ-ROSÉS, H.; SEBÉ, F. Synthetic generation of social network data with endorsements. In: eprint arXiv:1411.6273. [S.1.: s.n.], 2014. Citation on page 88.

PERROT, A.; AUBER, D. Cornac: Tackling Huge Graph Visualization with Big Data Infrastructure. IEEE transactions on big data, IEEE, v. 14, p. 1-1, 2018. Citations on pages 41 and 187.

PETERSEN, K.; FELDT, R.; MUJTABA, S.; MATTSSON, M. Systematic Mapping Studies in Software Engineering. 12Th International Conference on Evaluation and Assessment in Software Engineering, v. 17, p. 10, 2008. Citation on page 36.

PETRI, G.; SCOLAMIERO, M.; DONATO, I.; VACCARINO, F. Topological strata of weighted complex networks. PLOS ONE, v. 8, n. 6, p. 1-8, 06 2013. Citation on page 193.

PHAM, M.-D.; BONCZ, P.; ERLING, O. S3g2: A scalable structure-correlated social graph generator. Proceedings in Selected Topics in Performance Evaluation and Benchmarking: 4th TPC Technology Conference, n. August, p. 222, 2013. Citation on page 88.

PHAM, P.; DO, P. W-metapath2vec: The topic-driven meta-path-based model for large-scaled content-based heterogeneous information network representation learning. Expert Systems with Applications, Elsevier, v. 123, p. 328-344, 2019. Citation on page 71.

PONNUSAMY, R.; MANSOUR, N.; A., C.; FOX, G. C. Graph contraction for mapping data on parallel computers: A quality-cost tradeoff. Scientific Programming, v. 3, p. 73-82, 1994. Citations on pages 38 and 41.

PUROHIT, M.; PRAKASH, B. A.; KANG, C.; ZHANG, Y.; SUBRAHMANIAN, V. S. Fast influence-based coarsening for large networks. In: Proceedings of the ACM SIGKDD International Conference on Knowledge Discovery and Data Mining (KDD). [S.l.: s.n.], 2014. p. 1296-1305. Citations on pages 55 and 77.

RABBANY, R.; TAKAFFOLI, M.; FAGNAN, J.; ZAÏANE, O. R.; CAMPELLO, R. J. G. B. Communities validity: methodical evaluation of community mining algorithms. Social Network Analysis and Mining, v. 3, n. 4, p. 1039-1062, 2013. Citation on page 86. 
RAGHAVAN, U. N.; ALBERT, R.; KUMARA, S. Near linear time algorithm to detect community structures in large-scale networks. Phys. Rev. E, v. 76, p. 036106, Sep 2007. Citations on pages 57, 80, 170, 176, and 183.

REES, B. S.; GALLAGHER, K. B. Overlapping community detection using a community optimized graph swarm. Social Network Analysis and Mining, v. 2, n. 4, p. 405-417, 2012. Citation on page 86.

RODNEY, D.; SOPER, A.; WALSHAW, C. The application of multilevel refinement to the vehicle routing problem. In: IEEE. Proceedings of the IEEE Symposium on Computational Intelligence in Scheduling (CISsched). [S.1.], 2007. p. 212-219. Citation on page 135.

RON, D.; SAFRO, I.; BRANDT, A. Relaxation-based coarsening and multiscale graph organization. Multiscale Modeling \& Simulation, v. 9, n. 1, p. 407-423, 2011. Citation on page 39.

ROSSI, R. G.; LOPES, A. A.; FALEIROS, T. de P.; REZENDE, S. O. Inductive model generation for text classification using a bipartite heterogeneous network. Journal of Computer Science and Technology, v. 29, n. 3, p. 361-375, 2014. Citations on pages 24 and 162.

ROSSI, R. G.; LOPES, A. de A.; REZENDE, S. O. Optimization and label propagation in bipartite heterogeneous networks to improve transductive classification of texts. Information Processing and Management, v. 52, n. 2, p. 217 - 257, 2016. ISSN 0306-4573. Citations on pages 115 and 129.

Rosvall, M.; Delvenne, J.-C.; Schaub, M. T.; Lambiotte, R. Different approaches to community detection. arXiv e-prints, p. arXiv:1712.06468, 2017. Citation on page 93.

ROTTA, R.; NOACK, A. Multilevel local search algorithms for modularity clustering. Journal of Experimental Algorithmics, v. 16, n. 2, p. 2.1, 2011. Citations on pages 116 and 135.

SAFRO, I.; RON, D.; BRANDT, A. Graph minimum linear arrangement by multilevel weighted edge contractions. Journal of Algorithms, v. 60, n. 1, p. 24-41, 2006. Citations on pages 41, 55 , and 77.

SAFRO, I.; SANDERS, P.; SCHULZ, C. Advanced coarsening schemes for graph partitioning. In: Proceedings of the International Symposium on Experimental Algorithms (SEA). [S.1.: s.n.], 2012. v. 7276 LNCS, p. 369-380. Citations on pages 55 and 76.

SAKELLARIDI, S.; FANG, H. R.; SAAD, Y. Graph-based multilevel dimensionality reduction with applications to eigenfaces and latent semantic indexing. In: Proceedings of the International Conference on Machine Learning and Applications (ICMLA). [S.1.: s.n.], 2008. p. 194-200. Citations on pages 41 and 68.

SANDERS, P.; SCHULZ, C. Engineering multilevel graph partitioning algorithms. In: Proceedings of the European Symposium on Algorithms (ESA). [S.1.: s.n.], 2011. v. 6942 LNCS, p. 469-480. Citations on pages 39, 55, and 75 .

SCHLOEGEL, K.; KARYPIS, G.; KUMAR, V. Parallel multilevel algorithms for multi-constraint graph partitioning. In: Proceedings of the International Euro-Par Conference. [S.1.: s.n.], 2000. v. 1900, p. 296-310. Citation on page 135. 
SCHUETZ, P.; CAFLISCH, A. Efficient modularity optimization by multistep greedy algorithm and vertex mover refinement. Physical Review E - Statistical, Nonlinear, and Soft Matter Physics, v. 77, n. 4, p. 1-7, 2008. Citations on pages 41, 57, 64, 79, 116, and 135.

Multistep greedy algorithm identifies community structure in real-world and computergenerated networks. Physical Review E, v. 78, p. 026112, 2008. Citations on pages 57, 79, and 80 .

SCHWEITZ, E. A.; AGRAWAL, D. P. A parallelization domain oriented multilevel graph partitioner. IEEE Transactions on Computers, v. 51, n. 12, p. 1435 - 1441, 2007. Citations on pages 116 and 135 .

SCOTT, J. Social Network Analysis: A Handbook. [S.1.]: SAGE Publications, 2000. ISBN 9780761963394. Citation on page 23.

SETH, A.; ZHANG, J. A social network based approach to personalized recommendation of participatory media content. In: ICWSM. [S.1.: s.n.], 2008. Citation on page 26.

SHI, C.; LI, Y.; ZHANG, J.; SUN, Y.; YU, P. S. A survey of heterogeneous information network analysis. IEEE Transactions on Knowledge and Data Engineering, v. 29, n. 1, p. 17-37, 2017. Citations on pages 71 and 202.

SHI, C.; LI, Y.; ZHANG, J.; SUN, Y.; PHILIP, S. Y. A survey of heterogeneous information network analysis. IEEE Transactions on Knowledge and Data Engineering, IEEE, v. 29, n. 1, p. 17-37, 2017. Citation on page 110 .

SIDDIQUI, A. M.; GHAFOOR, A.; KHOKHER, M. R. Image segmentation using multilevel graph cuts and graph development using fuzzy rule-based system. IET Image Processing, v. 7, n. 3, p. 201-211, 2013. Citation on page 41.

SOUAM, F.; AITELHADJ, A.; BABA-ALI, R. Dual modularity optimization for detecting overlapping communities in bipartite networks. Knowledge and Information Systems, v. 40, n. 2, p. 455-488, 2014. Citation on page 87.

SPORNS, O. Networks analysis, complexity, and brain function. Complex., v. 8, n. 1, p. 56-60, Sep. 2002. Citation on page 23.

STRAM, R.; REUSS, P.; ALTHOFF, K.-D. Weighted one mode projection of a bipartite graph as a local similarity measure. In: SPRINGER. International Conference on Case-Based Reasoning. [S.1.], 2017. p. 375-389. Citation on page 26.

Dynamic case bases and the asymmetrical weighted one-mode projection. In: SPRINGER. International Conference on Case-Based Reasoning. [S.1.], 2018. p. 385-398. Citation on page 26.

SUARIS, P. R.; KEDEM, G. An algorithm for quadrisection and its application to standard cell placement. IEEE Transactions on Circuits and Systems, v. 35, n. 3, p. 294-303, 1988. Citation on page 64.

TENG, S.-H. Coarsening, sampling, and smoothing: Elements of the multilevel method. Algorithms for Parallel Processing, v. 105, p. 247-276, 1999. Citations on pages 24, 34, 38, 41, and 130 . 
THÉBAULT, E. Identifying compartments in presence-absence matrices and bipartite networks: Insights into modularity measures. Journal of Biogeography, v. 40, n. 4, p. 759-768, 2013. Citation on page 116.

TOLLIVER, D.; COLLINS, R. T.; BAKER, S. Multilevel spectral partitioning for efficient image segmentation and tracking. In: Proceedings of the IEEE Workshop on Applications of Computer Vision (WACV). [S.1.: s.n.], 2007. p. 414-420. Citation on page 41.

TOOSI, F. G.; NIKOLOV, N. S. Vertex-neighboring multilevel force-directed graph drawing. In: Proceedings of the IEEE International Conference on Systems, Man, and Cybernetics (SMC). [S.1.: s.n.], 2016. p. 002996-003001. Citation on page 41.

TRIFUNOVIC, A.; KNOTTENBELT, W. J. A parallel algorithm for multilevel k-way hypergraph partitioning. In: Proceeding of the International Symposium on Parallel and Distributed Computing, Workshop on Algorithms, Models and Tools for Parallel Computing on Heterogeneous Networks. [S.1.]: IEEE, 2004. p. 114-121. Citations on pages 116 and 135.

Parkway 2.0: A parallel multilevel hypergraph partitioning tool. In: Proceedings of the International Symposium on Computer and Information Sciences. [S.1.: s.n.], 2004. p. 789800. Citations on pages 116 and 135.

UBARU, S.; SAAD, Y. Sampling and multilevel coarsening algorithms for fast matrix approximations. Numerical Linear Algebra with Applications, v. 26, n. 3, p. e2234, 2017. Citation on page 41 .

USLU, T.; MEHLER, A. PolyViz: a visualization system for a special kind of multipartite graphs. In: Proceedings of the IEEE VIS 2018. [S.1.: s.n.], 2018. Accepted. Citation on page 113.

VALEJO, A.; DRURY, B.; VALVERDE-REBAZA, J.; LOPES de Alneu de A. Identification of related brazilian portuguese verb groups using overlapping community detection. In: SPRINGER, CHAM. Proceeding of the International Conference on Computational Processing of the Portuguese Language. [S.1.], 2014. p. 292-297. Citations on pages 86 and 116.

VALEJO, A.; FERREIRA, V.; OLIVEIRA, M. C. F.; LOPES, A. A. Community detection in bipartite network: A modified coarsening approach. In: Communications in Computer and Information Science (CCIS). [S.1.]: Springer International Publishing, 2018. v. 795, p. 123-136. Citations on pages 24, 30, 40, 55, 71, 162, 166, and 167.

VALEJO, A.; FILHO, G. P. R.; OLIVEIRA, M. C. F.; ; LOPES, A. A. Multilevel approach for combinatorial optimization in bipartite network. Knowledge-based Systems (KNOSYS), 2018. Citations on pages 30, 40, 41, 42, 50, 56, 63, 66, 68, 71, 158, 162, 166, and 167.

VALEJO, A.; GOES, F.; ROMANETTO, L. M.; OLIVEIRA, M. C. F.; LOPES, A. A. A benchmarking tool for the generation of bipartite network models with overlapping communities. Knowledge and information systems, accepted paper, 2019. Citations on pages 175, 179, and 182.

VALEJO, A.; GóES, F.; ROMANETTO, L.; OLIVEIRA, M. C. F.; LOPES, A. A. A benchmarking tool for the generation of bipartite network models with overlapping communities. Knowledge and Information Systems, accepted paper, v. 1, n. 1, p. 21, Jan 2018. Citation on page 29. 
VALEJO, A.; OLIVEIRA, M. C. R. F.; FILHO, G. P.; LOPES, A. A. Multilevel approach for combinatorial optimization in bipartite network. Knowledge-based systems, v. 151, p. 45-61, 2018. Citation on page 86 .

VALEJO, A.; V., F.; FILHO, G. P. R.; OLIVEIRA, M. C. F.; ; LOPES, A. A. One-mode projectionbased multilevel approach for community detection in bipartite networks. In: Proceedings of the International Symposium on Information Management and Big Data (SIMBig). [S.1.: s.n.], 2017. Citations on pages 30, 40, 42, 55, 71, 117, 158, 162, 166, and 167.

VALEJO, A.; VALVERDE-REBAZA, J.; DRURY, B.; LOPES, A. A. Multilevel refinement based on neighborhood similarity. In: Proceedings of the International Database Engineering $\backslash$ \& Applications Symposium. [S.1.: s.n.], 2014. p. 67-76. Citations on pages 24, 64, 116, 130, and 135 .

Multilevel refinement based on neighborhood similarity. In: Proceedings of the International Database Engineering \& Applications Symposium (IDEAS). New York, NY, USA: ACM, 2014. (IDEAS '14), p. 67-76. Citation on page 164.

VALEJO, A.; VALVERDE-REBAZA, J.; LOPES, A. A. A multilevel approach for overlapping community detection. In: Proceedings of the Brazilian Conference on Intelligent Systems. [S.1.: s.n.], 2014. p. 390-395. Citations on pages 24, 41, 116, and 130.

A multilevel approach for overlapping community detection. In: Proceedings of the Brazilian Conference on Intelligent Systems (BRACIS). [S.1.: s.n.], 2014. p. 390-395. Citation on page 164 .

VALEJO, A.; VALVERDE-REBAZA, J. C.; LOPES, A. de A. A multilevel approach for overlapping community detection. In: Proceedings of the Brazilian Conference on Intelligent Systems (BRACIS). Berlin: Springer, 2014. Citation on page 86.

WALSHAW, C. A multilevel approach to the travelling salesman problem. Operations Research, v. 50, n. September 2016, p. 862-877, 2000. Citation on page 135.

. A multilevel algorithm for force-directed graph drawing. In: Proceedings of the International Symposium on Graph Drawing. [S.1.: s.n.], 2001. v. 1984, n. 3, p. 171-182. Citations on pages $24,41,130$, and 135 .

A Multilevel Approach to the Graph Colouring Problem. [S.1.], 2001. Citations on pages $41,55,68,69$, and 135 .

A Multilevel Lin-Kernighan-Helsgaun Algorithm for the Travelling Salesman Problem. [S.1.], 2001. Citation on page 41.

Multilevel refinement for combinatorial optimisation problems. Annals of Operations Research, v. 131, n. 1-4, p. 325-372, 2004. Citations on pages 34, 40, 41, 44, 55, 68, 69, 130, and 134.

Hybrid metaheuristics: An emerging approach to optimization. In: . [S.1.]: Springer Berlin Heidelberg, 2008. v. 114, chap. Multilevel refinement for combinatorial optimisation: Boosting metaheuristic performance, p. 261-289. Citations on pages 130 and 134.

Multilevel refinement for combinatorial optimisation: Boosting metaheuristic performance. Hybrid Metaheuristics, v. 114, p. 261-289, 2008. Citations on pages 34, 40, 41, 55, 68, and 69. 
WALSHAW, C.; CROSS, M. Mesh partitioning : a multilevel balancing and refinement algorithm. SIAM Journal on Scientific Computing (SISC), v. 22, n. 1, p. 63-80, 1998. Citations on pages 41 and 134 .

Mesh partitioning: A multilevel balancing and refinement algorithm. Journal on Scientific Computing (SIAM), v. 22, n. 1, p. 63-80, 2000. Citations on pages 41 and 42.

Parallel optimisation algorithms for multilevel mesh partitioning. Parallel Computing, v. 26, n. i, p. 1635-1660, 2000. Citation on page 41.

JOSTLE: Parallel multilevel graph-partitioning software - an overview. [S.1.], 2007. 27-58 p. Citations on pages 41 and 135.

WALSHAW, C.; EVERETT, M. G. Multilevel landscapes in combinatorial optimisation. [S.1.], 2002. 1-21 p. Citations on pages 25, 40, 41, 130, and 135.

WANG, F.; ZHANG, C. Fast multilevel transduction on graphs. In: Proceedings of The SIAM Conference on Data Mining. [S.1.: s.n.], 2007. p. 157-168. Citation on page 41.

WANG, L.; XIAO, Y.; SHAO, B.; WANG, H. How to partition a billion-node graph. In: Proceedings of the International Conference on Data Engineering. [S.1.: s.n.], 2014. p. 568-579. Citation on page 57.

WANG, Z.; ZHANG, D.; ZHOU, X.; YANG, D.; YU, Z.; YU, Z. Discovering and profiling overlapping communities in location-based social networks. IEEE Transactions on Systems, Man, and Cybernetics: Systems, v. 44, n. 4, p. 499-509, 2014. ISSN 2168-2216. Citations on pages 24 and 130 .

WATTS, D. J.; STROGATZ, S. H. Collective dynamics of 'small-world' networks. Nature, v. 393, n. 6684, p. 440-2, 1998. Citations on pages 23, 24, 44, 115, 129, and 132.

Wong, P. C.; Mackey, P.; Cook, K. A.; Rohrer, R. M.; Foote, H.; Whiting, M. A. A multi-level middle-out cross-zooming approach for large graph analytics. In: 2009 IEEE Symposium on Visual Analytics Science and Technology. [S.1.: s.n.], 2009. p. 147-154. Citations on pages 41 and 187.

XIAOJIN, Z.; ZOUBIN, G. Learning from labeled and unlabeled data with label propagation. Tech. Rep., Technical Report CMU-CALD-02-107, Carnegie Mellon University, 2002. Citation on page 176.

XU, K.; WANG, F.; GU, L. Behavior analysis of internet traffic via bipartite graphs and onemode projections. IEEE/ACM Transactions on Networking (TON), IEEE Press, v. 22, n. 3, p. 931-942, 2014. Citation on page 26.

YANG, Z.; PEROTTI, J. I.; TESSONE, C. J. Hierarchical benchmark graphs for testing community detection algorithms. Physical Review E, American Physical Society, v. 96, p. 052311, Nov 2017. Citations on pages 86 and 89.

YAO, L.; WANG, Z.; LI, Z.; CAO, W.; WANG, Y. A novel coarsening scheme for multilevel graph partitiong. In: Proceedings of the International Conference on Biomedical Engineering and Informatics (BMEI). [S.1.: s.n.], 2011. p. 2091-2094. Citation on page 58. 
YE, Z.; HU, S.; YU, J. Adaptive clustering algorithm for community detection in complex networks. Physical Review E, American Physical Society, v. 78, p. 046115, 2008. Citations on pages 64,116 , and 135 .

ZENG, A.; SHEN, Z.; ZHOU, J.; WU, J.; FAN, Y.; WANG, Y.; STANLEY, H. E. The science of science: From the perspective of complex systems. Physics Reports, Elsevier, v. 714, p. 1-73, 2017. Citation on page 33.

ZHANG, C.; WANG, F. A multilevel approach for learning from labeled and unlabeled data on graphs. Pattern Recognition, Elsevier, v. 43, n. 6, p. 2301-2314, 2010. Citations on pages 41 and 135 .

ZHANG, Z.-Y.; AHN, Y.-Y. Community detection in bipartite networks using weighted symmetric binary matrix factorization. International Journal of Modern Physics C, v. 26, p. 1-14, 2015. Citations on pages 87 and 130.

ZHONG, E.; FAN, W.; ZHU, Y.; YANG, Q. Modeling the dynamics of composite social networks. In: ACM. Proceedings of the 19th ACM SIGKDD international conference on Knowledge discovery and data mining. [S.1.], 2013. p. 937-945. Citation on page 111.

ZHOU, T.; REN, J.; MEDO, M.; ZHANG, Y.-C. Bipartite network projection and personal recommendation. Physical Review E, APS, v. 76, n. 4, p. 046115, 2007. Citation on page 26.

ZHOU, W.; HUANG, J. A fast multi-level algorithm for drawing large undirected graphs. In: Proceedings of the International Conference on Internet Computing in Science and Engineering (ICICSE). [S.1.: s.n.], 2008. p. 110-117. Citation on page 41.

ZHOUA, K.; MARTIN, A.; PAN, Q.; LIU, Z. Evidential label propagation algorithm for graphs. In: Proceedings of the International Conference on Information Fusion (FUSION). [S.1.: s.n.], 2016. p. 1316-1323. Citation on page 176.

ZHU, M.; MENG, F.; ZHOU, Y.; YUAN, G. An approximate spectral clustering for community detection based on coarsening networks. International Journal of Advancements in Computing Technology, v. 4, n. 4, p. 235-243, 2012. Citation on page 53.

ZWEIG, K. A.; KAUFMANN, M. A systematic approach to the one-mode projection of bipartite graphs. Social Network Analysis and Mining, v. 1, n. 3, p. 187-218, Jul 2011. Citations on pages 25 and 166. 


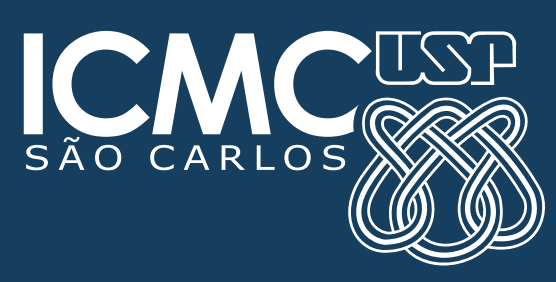

